\title{
KNOWLEDGE SHARING, MAINTENANCE, AND USE IN ONLINE SUPPORT COMMUNITIES
}

\author{
by \\ Derek L. Hansen \\ A dissertation submitted in partial fulfillment \\ of the requirements for the degree of \\ Doctor of Philosophy \\ (Information) \\ in The University of Michigan \\ 2007
}

Doctoral Committee:

Professor Paul J. Resnick, Chair

Associate Professor Mark S. Ackerman

Assistant Professor Caroline R. Richardson

Assistant Professor Soo Young Rieh 


\section{(C) DEREK HANSEN}

All rights reserved

2007 
For Maren 


\section{ACKNOWLEDGEMENTS}

I am deeply appreciative of the many individuals who have helped shape this dissertation and continually encouraged me throughout its writing. I hope that each of them feels some sense of shared ownership of this work and is pleased to be associated with it. Although I can’t mention everyone by name, a few are worth special notice.

I owe much to my advisor Paul Resnick, who has played an instrumental role in my education since the day I showed up at SI. I appreciate his early confidence in my abilities as I transitioned into the doctoral program and his continual expectations of excellence. He has been a tireless champion, reviewer, and source of insight throughout. He is one of the few truly interdisciplinary researchers I know and has a keen way of getting to the heart of any issue. Perhaps more than anything, I appreciate his personal integrity and willingness to impartially consider all sides of each issue.

I also appreciate the insights provided by my committee members. I have benefited greatly from Mark Ackerman’s enthusiasm for my work and guidance in performing effective interviews and textual analysis. I also appreciated his comments on papers we co-authored and insights into the publication process and academic life more generally. I hope that at least some of his many insights into the social world have bled into this work and my own thinking. I am thankful for my association with Soo Young Rieh who has introduced me to the world of information behavior research through our IBTheory group discussions. Her curiosity and openness to new ideas is contagious. Caroline Richardson has been a constant source of personal encouragement since I worked with her my first year at SI. She has been my window into the medical arena and has helped me think more broadly about the implications of my work. 
Jeff Mackie-Mason and the STIET program that he has overseen have challenged me to consider ways that my work relates to the work occurring in related disciplines. The funding I received as a STIET fellow allowed me to follow my passion and study an online medical support group early in the program, a study that ultimately led to my dissertation topic.

I have also benefited greatly from my conversations and friendships with many doctoral students, of which there are too many to name. I look forward to many years of future collaborations. I benefited greatly from the help of Sean Munson who helped create the wiki repositories we used in our action research project and make them more user-friendly. He was also a tireless content rater and soundingboard. His energy and efficiency helped reinvigorate me. I also appreciate the help of Erin Gong in formatting this document, and fighting with Endnote so I didn’t have to.

I have been overwhelmed with the helpfulness of the communities that I have worked with as part of this dissertation including css-discuss, GSI, and the Mediboard Graves' Disease and Chronic Pain groups. The administrators of each community have been enourmously helpful and responsive to my needs as a researcher, despite their extensive volunteer efforts in running the communities. I also appreciate Incutio Limited for providing access to the css-discuss server logs.

Finally, I would like to thank my family. Maren has been a constant source of insight as a practicing information glutton, especially in the medical arena. She has always chosen to see the best in me and inspired me to reach my potential. I will always remember her selfless sacrifices in supporting me throughout these years in Michigan, even as our family grew from one daughter to four. And thank you Adia, Sedona, Brynn, and Ashlinn for making the journey so enjoyable and meaningful.

This work has been funded by the following National Science Foundation grants: IRI-9702904, IIS-0325837, IIS-0308006, and 0114368. 


\section{TABLE OF CONTENTS}

Dedication $\quad$ ii

Acknowledgements iii

List of Figures $\quad$ vi

List of Tables vii

List of Appendices viii

Chapter

1. INTRODUCTION 1

2. CONVERSATION AND COMMUNITY REPOSITORIES IN 12 ONLINE SUPPORT COMMUNITIES

3. CSS-D STUDY INTRODUCTION \& METHODS 56

4. CSS-D EMAIL LIST 82

5. CSS-D WIKI REPOSITORY 127

6. CSS-D EMAIL LIST \& WIKI COMPLEMENTARITIES 172

7. CSS-D PRACTICAL LESSONS LEARNED 222

8. ONLINE MEDICAL SUPPORT COMMUNITIES 230

9. MEDSHELF - INTRODUCING A WIKI REPOSITORY 253

10. CONCLUSIONS 304

Appendices $\quad 322$

$\begin{array}{ll}\text { Bibliography } & 378\end{array}$ 


\section{LIST OF FIGURES}

Figure

1.1: Dissertation Chapters Outline $\quad 10$

5.1: css-d Wiki Content Over Time 130

5.2: Unique css-d Wiki Visitors Over Time 131

5.3: UnderstandingWiki Page 132

5.4: UnderstandingWiki Page (Edit View Excerpt) 135

5.5: FrontPage (Excerpt) 136

5.6: RecentChanges Page (Excerpt) 138

5.7: ThreeColumnLayouts Page (Excerpts) 148

5.8: CssEditors (Excerpt) 149

5.9: ToHackOrNotToHack (Excerpts) 155

5.10: UsingFontSize (Excerpt) 156

6.1: Percent of All css-d List Messages Referencing the Wiki 179

6.2: Percent of All css-d Emails vs. Wiki Emails by Posting Frequency 180

9.1: Medshelf Main Page (Graves’ Disease Wiki) 271 


\section{LIST OF TABLES}

Table

2.1: Online Community Repository Comparison 37

3.1: css-d Interview Sample $\quad 69$

5.1: css-d Wiki Page Statistics 143

5.2: css-d Wiki Pages Most Often Accessed via Search Engines 145

5.3: css-d Wiki Page Genres 147

6.1: Reasons for Referencing the css-d Wiki \& Email List Archives 181

6.2: Most Referenced css-d Wiki Pages 183

7.1: Suggestions for Implementing a Wiki Repository 228

9.1: Medshelf Recruitment by Source 263

9.2: Medshelf Wiki Seeding Experience 283

9.3: Medshelf Wiki Contribution Experience after Initial Seeding 288

9.4: New Wiki Content Growth (Including Seeding Experience) 298 


\section{LIST OF APPENDICES}

Appendix

1. CSS-D TIMELINE 322

2. CSS-D CODE BOOK 323

3. MEDSHELF.ORG STARTUP MATERIALS 329

4. COMMUNITY RECRUITMENT EMAILS 373 


\section{CHAPTER 1 INTRODUCTION}

Widespread adoption of information technologies has fostered new social arrangements and opportunities for large-scale collaboration. Many of these new collaborations occur in online support communities where members voluntarily share their knowledge with one another on a particular topic of interest. These communities cover nearly every topic imaginable ranging from cancer treatment to website design to auto repair. These help-based communities fill a unique niche in the information landscape by sharing information that is difficult to come by in books, manuals, coworkers, and other information resources. They provide access to rare expertise and cover material in great depth. They also provide access to peers with similar experiences and concerns who are able to empathize and provide social support. Because these

communities are interactive, their content can be clarified, peer reviewed, and customized to meet individuals’ personal needs.

One of the great things about online community conversations is that they can be “overheard” by others. This enables the knowledge that is made explicit through the ongoing discussion to benefit far more individuals than the discussion's immediate participants. The value of community conversations as a secondary resource is attested to by the large percentage of lurkers in online communities and the popularity of archived conversations (e.g., public email archives). Recognizing their value, many communities encourage new members to search for answers to their questions in the community archives prior to posting a message to the community.

Although online communities can be an important and unique source of information and social support for community members and outsiders, they must deal 
with several problematic tensions. The first tension relates to the challenge of supporting the needs of participants in the original conversation, as well as the needs of onlookers. Individuals who come to the discussion with an immediate need benefit from personalized responses couched in terms of the original need. However, highly contextualized discussions can be difficult for onlookers to apply to their own situation, expecially when the onlookers lack content expertise. Thus, there is a tension between the need to personalize content for the individual and the need to generalize content for the many onlookers.

A second tension originally raised by Preece (1999) relates to the efficient provision of information and the need to allow for social support. It is especially pertinent to highly empathetic communities (e.g., online medical support groups). Many communities encourage members to refer to FAQ documents or conversation archives before posting to the community so that other community members won't be repeatedly bothered by the same questions. However, this practice may inadvertently lower the provision of social support which is often provided alongside answers to questions (Preece 1999). Likewise, some communities stringently keep the discussion on-topic by discouraging members from sharing information that is not directly related to the community topic. This helps assure that members (and onlookers) don’t need to weed through irrelevant conversations, but may also prevent conversations from occuring that are important for strengthening social ties. Likewise, it may create an environment less suitable for intimate communication. Thus, there is a tension between supporting efficient communication and supporting meaningful social interactions.

Finally, there is an ongoing challenge in online communities of supporting the different needs of core participants and newcomers. Core participants are often most interested in keeping up-to-date on the newest developments and continuing friendships with other long-time community members. Meanwhile, many newcomers are primarily interested in having an immediate need met by the community. An overemphasis on 
meeting the needs of the newcomers can frustrate core members who feel like they must repeatedly answer the same, basic questions without receiving much benefit or advancing the conversation. Meanwhile, an overemphasis on meeting the needs of core members may frustrate newcomers who feel like they shouldn’t disrupt the ongoing conversation with their own personal problems.

One promising way of overcoming, or at least lessening these tensions is to augment the existing community conversation with information resources of a more lasting nature. Many communities have done this by creating community repositories such as FAQ documents and conversation archives. More recently, some communities have implemented a community repository by using a wiki, a type of online collaborative authoring tool. Wiki repositories have fostered new ways of sharing, maintaining, and using community knowledge, and when combined with community conversation they have the potential to overcome the problematic tensions outlined above. For example, the conversation could be used to meet individuals’ personalized needs, while the wiki repository could be used to distill the conversation into a more reusable and generalized form. Newcomers could use the wiki to help address their own questions when possible, and when not possible core members could more easily answer questions by referring questioners to relevant wiki pages. Although there is anecdotal support for these potential benefits, no empirical examination of an online support community with a wiki repository has been conducted. This makes it impossible to characterize the actual benefits and challenges of wiki repositories or understand how communities can best implement them.

The purpose of this dissertation is to help fill this gap in our understanding by meeting two related goals: one practical and one theoretical. The practical goal is to improve online support communities by helping them realize the potential of wiki repositories. It requires that I answer the following questions: What benefits and challenges do wiki repositories offer online support communities? What activities, social 
roles, genres, and norms help communities effectively implement and use a wiki repository? What communities are well suited (or poorly suited) for using a wiki repository?

The theoretical goal is to formulate general principles of inquiry related to the combination of conversation and documents of more lasting value (i.e., wiki repository pages). What are the strengths and weaknesses of each? What is the nature of their relationship? How can they be coupled together through social norms, community roles, and specific technological features? What work is required to maintain this coupling? In what ways do they draw from each other? How do differences in the community context affect these issues? I intend for this deeper understanding to help lay the groundwork for future research on this topic, as well as help inform potential novel design suggestions.

Both the practical and theoretical goals are best addressed through empirical examination of communities that have augmented their community conversation with a wiki repository. I use a two-phased approach in this dissertation. In the first phase I empirically examine an online support community that has successfully integrated a wiki repository with their email list discussion. The community, css-d, was chosen because of its apparent success, the availability and comprehensiveness of its data, and the fact that it was representative of many online support communities with its wide variation in member expertise, reliance on threaded conversation, and focus on question asking and answering within a particular domain. In the second phase I explore the importance of contextual factors, as well as validate the best practices identified in the css-d study. This is done through an action research project where I introduce a wiki repository to 3 existing online medical support communities. Medical support communities were chosen because of their prevalence, the potential for improving their members' well-being, the fact that they have been so well studied, and because they include so many potential barriers to implementing a wiki repository, such as privacy and quality concerns. 
My findings from these studies demonstrate how a threaded conversation and wiki repository can complement each other. For example, I show how the email list helped provide a critical number of contributors, as well as an existing social structure that helped focus attention and establish norms around the wiki. I also show how the email list provided topics, content, and motivation for members to contribute to the wiki, as well as feedback on the wiki content as it was used in the ongoing discussion. Conversely, I show how the wiki improved the email list by making question asking and answering easier because content could be reused (i.e., referenced) rather than recreated. Likewise, I show how the wiki helped overcome common governance challenges in the threaded conversation (e.g., dealing with off-topic conversation). I also show how it helped attract new members and retain long-time members. And finally, I show how it helped motivate high quality email list responses.

I also identify some of the considerations important in deciding if a community should adopt a wiki. These are based on the css-d study, as well as the action research project. My findings suggest that a community needs a sufficient number of active participants and a constant influx of new members to sustain a repository. The newcomers are important not because of their direct contributions to the wiki, but because their recurring questions help motivate long-time members to contribute to the wiki. Communities that deal with information that is highly distributed are excellent candidates for a wiki repository, which is ideal for summarizing and aggregating disparate information. As expected, privacy was an important consideration for several health communities, some of which use private email lists. However, communities saw the wiki as a way of attracting new members by presenting a public face to the outside world, something that is particularly important to private email lists which can’t attract members through their email archives. Although the lack of wiki experience and technical ability of members can dissuade initial widespread use, my experience with 
communities with little prior wiki experience (and other technical ability) demonstrated that these challenges were possible to overcome.

I also identify and test promising best practices for improving online support community email lists and wiki repositories, and suggestions for how to make them complement each other. For example, I show how the inclusion of off-topic material in the wiki helps to keep the discussion on-topic. I identify certain wiki genres such as the Annotated Links genre that are particularly well-suited for collaborative authoring in a wiki repository. I also demonstrate the importance of several specific practices such as having a small group of community members seed the wiki with initial content, promoting the wiki by linking to specific wiki pages often, establishing which activities should occur in the email list versus the wiki, and setting reasonable expectations for relatively slow wiki growth over time.

Finally, I identify challenges related to the introduction of the wiki and some suggestions for overcoming those challenges. One initial challenge is to identify content that should be included in the wiki to begin with. Overcoming this challenge requires members to see the wiki as a community resource, rather than a treatise on their community topic. Thus, initial wiki content should address frequently asked questions, hotly contested issues, and new developments that members are interested in following content that will be useful in referring to later in the conversation and of interest to many members. Another major challenge is encouraging members to contribute to the wiki. Having multiple members (i.e., a “wiki team”) seed the wiki with content may take longer initially (because of coordination challenges), but helps other members see the wiki as a community resource and helps train multiple wiki advocates who are likely to promote the wiki later on. Finally, another common challenge was a hesitancy to edit other members' contributions on the wiki.

\section{Summary of Contributions}


In this dissertation, I make a number of novel research contributions. To begin, I find that the very same characteristics that make community conversations so useful to their participants (e.g., their personalized, immediate, and social nature) make their reuse by onlookers problematic. Not only is valuable knowledge hidden among social interactions and conversations of no lasting value, it must be decontextualized and reapplied to the onlookers’ particular needs. This work of de-contextualizing and recontextualizing requires expertise that many onlookers do not posess, suggesting that even a perfect search algorithm applied to a conversation archive is insufficient for supporting conversation reuse.

I then show how the introduction of a wiki repository can help overcome the tension between supporting the needs of the immediate conversation participants and the needs of onlookers. The ability to collaboratively edit, organize, and structure content on the wiki allows members to perform new activities such as aggregating, summarizing, and refining content which can be encapsulated in new information genres such as Annotated Links, Debate summary pages, and Articles (see Chapter 5). Most of these wiki pages are created by experts so that they can later refer to them when answering frequently asked questions. This is nice because experts are the ones with the skills required to effectively decontextualize and summarize the highly personalized discussion.

The result of this process is that wiki content is created by experts on topics of interest to novices. Although experts create these resources primarily to save themselves time later, they are also ideal for outsiders and newcomers to learn from because they are conducive to reuse (e.g., decontextualized and summarized). Furthermore, as wiki content is reused in the conversation, it is maintained and improved. All of these benefits derive from the close coupling of the repository with the ongoing question and answer discussion including both novices and experts. Although it would seem reasonable to rely solely upon experts to create useful and high quality website content on a particular topic, these findings suggest that a better approach is to couple the content creation with a 
community conversation that includes novices and experts. The novices are important because they help signal topics of interest to the masses (through frequently asked questions), help motivate experts to make their knowledge explicit (when answering questions) and transfer it to the wiki, and identify problems with wiki content.

Not only does the combination of wiki repository and community conversation help overcome some of the information sharing and maintenance challenges, it also helps overcome some of the common social maintenance challenges of online communities. For example, I find that a wiki repository is useful in keeping the community discussion on-topic and avoiding pointless rehashing of potentially contentious debates (i.e., Holy Wars). Although communities may be inclined to avoid all off-topic content in a community repository, I find that its inclusion can help meet the needs of information seekers while discouraging off-topic conversation in the email list. These findings demonstrate that the social challenges and their potential solutions are highly affected by the information infrastructure and its use by members. In other words, decisions about how information can be shared, stored, and modified largely define the social challenges and opportunities of communities.

One of the key differences between a wiki repository and a traditional FAQ document is the fact that a wiki repository is easily editable by many people. The editable nature of a wiki repository facilitates the synergies between it and the community conversation. However, its editable nature also introduces some challenges that are not well recognized. After all, community authoring is a relatively new phenomenon that has only been enabled recently. Furthermore, I argue that it is not something we do naturally - like conversation. For example, I find that while deleting content is technically easier than adding content, it is socially far more challenging. Adding content only requires an assurance that at least one person will benefit from the content. Deleteing content, however, requires knowledge that the content was not useful to each of the community members. I also find that wiki content that is attributable to an individual (e.g., content 
directly copied from a community email list message) is less likely to be edited by other members. Thus providing attribution to a statement may be helpful in establishing credibility, while reducing its quality since it will not likely be maintained in the future. Both of these examples suggest that communities could benefit from establishing strong norms and expectations about collaborative authoring behaviors, although what those norms are remains a topic of future research.

Finally, in the conclusion (Chapter 10) I propose a theoretical framework to guide research into the relationship of community conversation and more persistent, community documents. Specifically, I propose that communities with a conversation and set of information resources be viewed as a symbiotic organism living in the broader information ecology. Viewing a community as a symbiotic relationship draws attention to the ways that the community conversation and information resources influence one another. Viewing a community as part of the broader information ecology draws attention to its unique informational role, as well as its interdependence on other communities and resources.

\section{Dissertation Outline}

The dissertation is organized into 10 chapters including this introductory chapter (i.e., Chapter 1). Figure 1.1 shows how the chapters relate to one another.

Figure 1.1 Dissertation Chapters Outline 


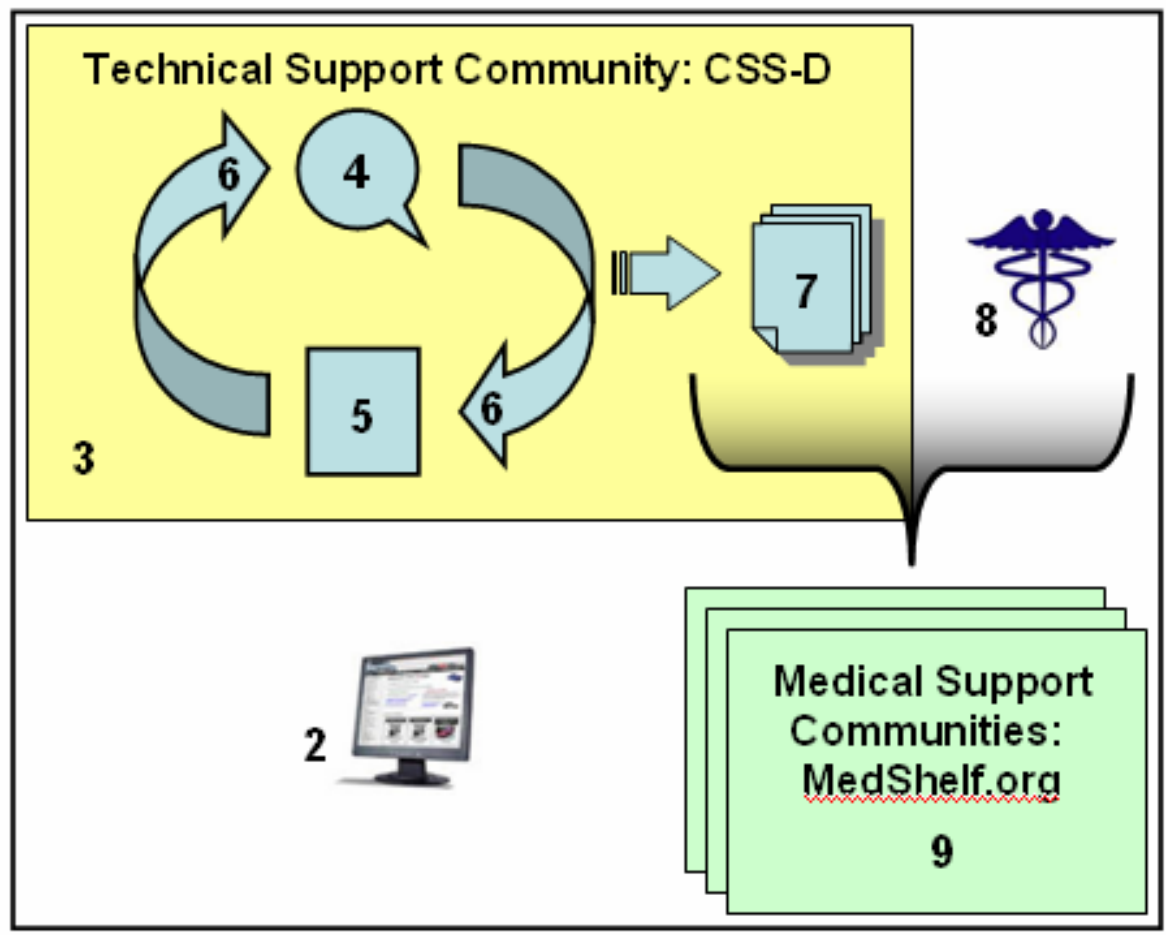

Chapter 2 is a literature review where I define online support communities and describe their key characteristics. I compare different types of community repositories (e.g., FAQ documents, email archives, and wiki repositories) and explore the potential role of a wiki repository in better supporting the needs of these communities. Thus, the chapter helps define the important issues and terminology used throughout the dissertation and relates them to prior research.

Chapters 3-7 are based on the empirical examination of css-d, a technical support community for website designers. Chapter 3 describes the research questions, available data, and methods used in the study. The key research question is: How is activity organized at css-d to leverage both the email list and wiki repository? Chapter 4 provides an in-depth look at the css-d email list. I provide a brief history of the community, describe the technology it uses, characterize the governance practices, describe the key social norms around reuse of information, identify the major communicative genres, and describe social roles and patterns of participation. I conclude the chapter by arguing that the characteristics that make the email list so useful for its active participants, are the 
same ones that make it less useful as a secondary resource. Chapter 5 mirrors chapter 4 by discussing the history, governance, social norms, technology, communicative genres, and social roles related to the wiki. I argue that certain activities (e.g., aggregating information) are better supported by the wiki, while other activities (e.g., diagnosing problems) are better supported by the email list. Chapter 6 describes the ways in which the email list and wiki help serve one another. It also provides a detailed look at the social practices used to couple the email list and wiki repository together. Chapter 7 summarizes the best practices gleaned from the css-d study into a collection of recommendations for communities interested in introducing a wiki repository.

Next, I discuss the action research project where online medical support communities were provided with a wiki repository infrastructure and suggestions on how to use it effectively. Chapter 8 is a literature review of online medical support communities that was needed to help translate the best practices derived from the css-d study (found in Chapter 7) into a medical support context. It characterizes the primary activities within these communities and compares them with css-d. Chapter 9 presents the results of the action research project. Using data from all solicited communities (i.e., those that ended up participating and those that did not), I identify the key factors that communities consider when deciding to adopt a wiki repository and discuss the implications of this on the types of communities that are good (or poor) candidates for a wiki repository. I also discuss how well the best practices from the css-d study worked and provide some idea of the importance of context in their application.

In Chapter 10, I discuss the implications of the findings on the design of interaction environments. This includes a discussion of the limitations of current design solutions intended to support information reuse, as well as some areas where novel design solutions are needed. I also discuss the unique role of online support communities in the broader information landscape. 


\section{CHAPTER 2}

\section{CONVERSATION AND COMMUNITY REPOSITORIES IN ONLINE SUPPORT COMMUNITIES}

Online communities are one of the most enduring legacies of the early Internet days. They are a testament of the human capacity to generate social context and meaning across time and space, even among strangers in a world of mere text. They represent one of the most widespread examples of what has more recently been coined "commonsbased peer production” where large groups of individuals voluntarily contribute towards the creation of public information goods and services (Benkler, 2002).

Traditionally, online communities have performed their work via asynchronous, threaded conversation in the form of email lists or online forums. The public, or semipublic, ongoing discussion was the primary peer-produced service. In many cases an archive of the discussion served as the primary information resource created by these communities, even if it was created as a byproduct with little intentional thought. More recently, some online communities have begun to augment their ongoing conversations by collaboratively authoring information resources for their own community use and for public consumption. The combination of large-scale conversations and collaborative authoring has the potential to transform online community participation and enable the creation of new community-driven information genres.

The purpose of this chapter is to define a particular type of online community, the online support community, and explore the potential role of community repositories (in conjunction with existing ongoing conversation) in supporting the needs of these communities. In the first section I define online support communities using Wenger's community of practice framework (1998). I also describe some of the current 
characteristics of these communities (e.g., threaded conversation design), common activities, and differentiating factors (e.g., community size). The second section takes a closer look at the role of community repositories. First I define community repository and differentiate three common types including a traditional FAQ, discussion archive, and wiki repository. Next, I explore the potential (and limitations) of community repositories, some of the challenges of implementing them effectively, and their underlying relationship to conversation.

\section{Online Support Communities}

Research into online communities has paralleled the growth of online communities themselves. Early work by Howard Rheingold emphasized the strong personal ties and well-established community norms that developed among members of the Well, one of the earliest versions of a virtual community of intellectuals, artists, and engineers (1993). Others, like Wellman and colleagues, have challenged the appropriateness of the word "community," laden with all of its nostalgic idealism, preferring instead to ditch the analogy of "place" (1999). Instead, they recommend using the more flexible and modern concept of social networks to describe these new social arrangements (Wellman, 1997). However, no consensus has been reached concerning the appropriateness of using these and other concepts and analogies to describe online communities. This may be in part due to the wide variety of online social arrangements that fly under the banner of "online community."

In a summary of research on virtual communities, Ellis, et al. argue that "it is impossible to provide one all-encompassing definition of virtual community” (2004, p 155). Why? Because the concept of virtual community is "open textured" meaning that it is "always corrigible or amendable" allowing for different parties to see what they want to see in it (Ellis et al., 2004). They quote Benders and Van Veen who argue that this flexibility or "interpretative viability" allows for concepts to be widely disseminated 
(2001). In the end, as a researcher we must carefully apply the concepts that we believe best illuminate the core realities, recognizing that some diversity of approach and terminology is inevitable and likely beneficial. However, it is also important to clearly define the concepts we are using so that we can recognize the boundaries of their applicability. In other words, clearly defined terminology and concepts help us make reasonable decisions about the applicability of our findings to other situations, even if they are called by different names. With this in mind, I begin by defining and listing the key characteristics of online support communities in the following section.

\section{Defining Online Support Communities}

Online support communities are a subset of the many types of online social arrangements that are typically referred to as online or virtual communities. In a review of the various definitions of virtual communities, Lee, et al. identify the common themes among various authors and propose the following definition of a virtual community: "a cyberspace supported by computer-based information technology, centered upon communication and interaction of participants to generate member-driven contents, resulting in a relationship being built up” (2003, p 4). This definition is fairly broad and would include a variety of social arrangements such as the Well, Wikipedia, Flickr, many Usenet groups, and possibly even sites such as eBay (although relationships are less likely to develop there than the other settings mentioned). This broad definition of virtual community is sufficient for our purposes and, as will be seen, clearly encompasses the more narrowly defined online support community.

No agreed upon typology of virtual communities exists. The significant differences in how researchers have classified the various types of virtual communities have been outlined by others (Lee et al., 2003; Porter, 2004). Classifications are derived from differences in basic human needs that are met (e.g., interest, relationship, fantasy, transaction), the technologies used by the communities (e.g., email list, forum, chat, 
MUD), the motivation of participants (e.g., a shared practice, purpose, circumstance, or interest), the content domain (e.g., geographic, business type, demographic), and the social structures in place (e.g., virtual settlements, cyber-inns, virtual airport bar) (Lee et al., 2003). Porter recommends differentiating communities based upon who initiated them (member-initiated, organization-sponsored) and the orientation of the relationships that form (e.g., social, professional, commercial) (2004).

While considerable effort has been expended in creating all-encompassing typologies of virtual communities, so far no agreed upon typology has been accepted. Fortunately, a comprehensive, unified typology is not necessary to perform meaningful research on virtual communities, or to define useful sub-types. Lee, et al. suggest that researchers adopt their own classification scheme depending on their research focus (2003). Others have avoided the issue in part by describing a few popular types of online communities, rather than providing an exhaustive typology. For example, Preece and Maloney-Krichmar describe patient support, education, and e-business communities (2003).

So where do online support communities fit within this mélange of typologies? There is no easy answer. I take a practical approach similar to that taken by the authors mentioned above and characterize online support communities by describing prototypical examples and sketching out the boundaries, no matter how grey they seem at times. To begin with, I will look at each word separately, although for expositional purposes they will not be treated in order.

\section{Online}

In this context, the term online indicates that the interaction is facilitated by a network, is computer-mediated, and occurs between people who are not all co-located. A canonical example of online interaction is a group of dispersed individuals holding a 
conversation via a threaded discussion board or a chat room. However, it is clear that many other situations are suggested by our definition of online.

I do not restrict the term online to mean on-the-Internet. In fact, many online communities thrive on private networks such as corporate Intranets or on mobile phone networks. In addition, as communication devices such as mobile phones have become more sophisticated, they have taken on much of the functionality originally performed by traditional computers. Thus, the restriction that the interaction must be computermediated should not be interpreted as desktop-mediated. Rather, it should imply that the communication device provides a meaningful representation, storage capability, or computation related to the conversation. Finally, this definition excludes technologies that facilitate interactions among entirely co-located groups (e.g., meeting support systems). There may be some individuals of a community that are co-located, but the majority of the members are generally not.

\section{Community}

As social practices have changed throughout the decades, so has the definition of community. As already discussed, the application of the term to online communities and the inherent analogies that it invokes have not been universally accepted (Wellman, 1997). However, I use the term for two reasons. First, the term “online community” is widely used and accepted in both practice and research. Because of its "interpretive viability" most people do not think it carries with it all of the baggage that Wellman initially feared. Second, as will be explained below, I want to explicitly link it to the communities of practice theoretical framework developed by Lave and Wenger (Lave \& Wenger, 1991; Wenger, 1998). This framework describes a specific type of social arrangement that captures the core of what I hope to convey through the use of the term “community.” A number of other authors have successfully applied this framework to online situations, despite the fact that it was originally developed by studying co-located 
groups (e.g., Kimble, Hildreth, \& Wright, 2001), although not all researchers agree that this is appropriate (see Ellis et al., 2004 for a summary; Lueg, 2000).

In his book Communities of Practice: Learning, Meaning, and Identity, Wenger identifies three dimensions that are essential to a community of practice. First, a community of practice has a shared and mutually negotiated understanding of what constitutes a joint enterprise (Wenger, 1998). This joint enterprise is largely implicit and is “defined by the participants in the very process of pursuing it” (1998, p 77). It is the community's raison d'être and, as such, defines the identity of the community by creating a shared sense of accountability to a body of knowledge (Wenger, McDermott, \& Snyder, 2002).The negotiation of a joint enterprise determines "what matters and what does not, what is important and why it is important, what to do and not to do, what to pay attention to and what to ignore, what to talk about and what to leave unsaid, what to justify and what to take for granted, what to display and what to withhold, when actions and artifacts are good enough and when they need improvement or refinement.”

Second, members of a community of practice are tied together through mutual engagement. As individuals interact with one another, learn together, and build relationships, they develop a sense of belonging and mutual commitment (Wenger et al., 2002). Membership in a community of practice is determined by engagement with other community members, not by geography, a shared title, or a social network (Wenger, 1998). Through mutual engagement with the community, "each member develops a unique individual identity in relation to the community” (Wenger et al., 2002, p 35).

Finally, each community of practice is further tied together by a shared repertoire, consisting of "routines, words, tools, ways of doing things, stories, gestures, symbols, genres, actions, or concepts that the community has produced or adopted in the course of its existence, and which have become part of its practice” (Wenger, 1998, p 83). This shared repertoire reflects the history of mutual engagement and serves as a resource that can be drawn on to further negotiate meaning. 
In summary, I will use the word community to suggest the core concepts captured by the communities of practice framework, rather than to suggest an analogy to idealized, nineteenth century towns. Thus, the term community suggests that there is ongoing, mutual engagement among a group of individuals with a shared repertoire that is continually negotiating its joint enterprise.

\section{Support}

The term "support" is used as an adjective to describe a certain type of community. As such, it shapes each of the three dimensions of community discussed above. It suggests a particular type of joint enterprise, where the primary focus is supporting the needs of individuals who are trying to make sense of, and deal with, a particular situation or knowledge domain (e.g., coping with a disease, working with a new technology, performing a job, joining an organization). These needs are generally highly contextualized and complex. It also suggests the types of mutual engagement that occur, which may include asking and answering questions, sharing resources, telling stories, soliciting or expressing empathy, and encouraging further education. Finally, it suggests certain elements of the shared repertoire that, although negotiated within each community, are nonetheless common among other support communities. For example, certain genres such as the Q\&A or "how to" threads, the use of textual smiley-faces, the expectation that members consult the archives before posting a question, are all typical of support communities.

It is important to recognize that I am breaking with tradition by using the term "support" in this broad sense (i.e., to capture social, emotional, and technical support). Many authors especially in the medical arena, reserve the term "support" exclusively for support groups. Similarly, the term "support" is drawn upon in open source projects to describe "technical support" or support of the software. Clearly these different historical and contextual meanings suggest important differences in the joint enterprises, mutual 
engagement, and shared repertoires of these communities. In fact, I devote Chapter 8 of this dissertation to the unique challenges and opportunities afforded by one particular type of online support community, namely online medical support communities. Findings from studies of one type of support community will need to be carefully considered before applying them to another type. However, despite these differences, there are many shared needs and practices between these and other online support communities that I believe warrant their joint consideration at times. In situations where I believe the particular type of online support community is important, I will use additional modifiers to clarify (e.g., online technical support community, online medical support community); otherwise I will use the more general term online support community.

\section{Putting it Together}

In summary, online support communities are distributed, computer-mediated communities of practice focused on supporting the needs of individuals who are trying to make sense of, and deal with, a particular situation or knowledge domain. This definition is broad enough to include most technical forums, medical support groups, helping communities (Wenger et al., 2002), and even communities of gamers supporting one another in their play. However, it excludes many types of online communities including transaction-based communities such as eBay and FreeCycle, social network communities like FaceBook and MySpace, and communities whose primary purpose is to create artifacts such as Wikipedia and Project Gutenberg. Although some of these communities provide various forms of support to their members (e.g., a free microwave or an introduction to a potential date), the primary thrust of participation within these communities is not on mutual support as related to a specific situation or knowledge domain. Thus, findings from studies of online support communities will need to be judiciously applied to these other forms of online communities, if at all. 


\section{Current Characteristics of Online Support Communities}

As it has been defined so far, there is still great flexibility in the look and feel of online support communities. I have said nothing about their size, their diversity, or the specific technologies that they employ. This is intentional, as it allows for a more diverse and enriching research agenda to be developed around them; one that can encompass current practices, as well as future possibilities. However, while the term may be more enduring and flexible in its application, it leaves the researcher with the task of filling in many of the details. This section and the following section help walk through some of those details. In this section I explain three important characteristics of online support communities as they exist at the present time: the threaded conversation design, the voluntary nature of participation, and the public nature of participation within those communities. In the next section I explain some of the core differences among the many variations of online support communities.

The characteristics outlined below describe what is commonly thought of when the term online support community is used. The characteristics are largely a result of historical processes, not underlying needs of online support communities. In fact, they are shared by many other online communities because of their shared history (Preece, Maloney-Krichmar, \& Abras, 2003). Even though these characteristics may change over time, they are vital to understanding the essence of online support communities in their current instantiation. Any practical recommendations on how to improve the current state of online support communities will need to recognize these characteristics and fit within the backdrop they provide.

\section{Threaded Conversation Design}

The vast majority of online support community discourse occurs via threaded conversation (see Lee et al. 2003 for some numbers on virtual communities more 
generally). Whether occurring via Usenet, email lists, or forums, threaded conversation has several important characteristics. The following list is taken largely from Resnick, Hansen, et al. (2005):

- Topics. A set of topics, groups, or spaces, sometimes hierarchically organized to aid users in discovering interesting groups to "join.” Topics/groups are persistent, though their contents may change over time.

- Threads. Within each topic or group, there are top-level messages and responses to those messages. Generally further nesting - responses to responses - is permitted. The top-level message and the entire tree of responses to it is called a thread.

- Permanence. Once a message is posted, it cannot be rewritten. A new message may be posted, but the visibility of the original message is unchanged.

- Homogenous view. The partitioning of messages into topics is shared by all viewers. Moreover, users all see the same view of the messages in a topic, either in chronological or reverse chronological order. In some cases, messages are sorted into threads, with chronological presentation of threads, and chronological presentation within threads. In some cases, the system will keep track of which messages a user has previously viewed, so that it can highlight the unread messages, but that is the only personalization of how people view the messages.

- No summarization. There are no structural features that identify relations between messages other than the "replies-to" relation. In particular, there are no mechanisms that flag certain messages as summaries of others and no special support for processes that produce such summaries.

- Author Association. Each message is ascribed to an author. In most cases the author is required to register, at which time they can choose a pseudonym that will be attached to their messages. In some systems an individual can post a message as a "guest" or anonymous user.

Despite its relatively simple structure, the threaded conversation design has proven very adaptable in serving a variety of purposes and user groups. Its familiarity makes it relatively easy for new members to find their bearings, at least from a technical standpoint. Its structural features make it easy to recognize who one is conversing with, although in most cases it is impossible to know who is listening in. It also allows 
members to select the conversations they will be interested in, albeit imperfectly since topic and message names don’t always reflect the actual conversation.

It is important to recognize the constraining influence of the technical infrastructure on the mutual engagement of the community and the ways that the shared repertoire is negotiated (e.g., Ackerman \& Palen, 1996; Erickson, 1997 provide examples of how the technical infrastructure influences social interaction). For example, threaded conversation (with its emphasis on turn-taking and permanence) encourages discussion, debate, and personal accountability. These same features deter other forms of participation such as selecting, emphasizing, aggregating, and summarizing. I am not suggesting that these activities cannot occur in a threaded conversation, indeed they do occur because of their importance. Instead, I am suggesting that they are not well supported by the threaded conversation design. When I enter the kitchen I instinctively look for food and, indeed, the very design of the cabinets and appliances makes it the perfect place to work with food. Although I can read a book or even take a nap in the kitchen if I want to, the thought rarely crosses my mind and the architecture is poorly suited for such activities. In a similar way, an individual may, for example, post a message that summarizes an earlier thread, but the summary message will quickly be buried among a host of others, making the design less than ideally suited for the task of summarizing information for future reuse.

\section{Voluntary Participation}

The vast majority of members of online support communities participate voluntarily. Both their membership and their level of contribution to the community are individually determined with little compulsion from others outside or inside the community. Although some online support communities include participants or community leaders who are paid to participate, most members join and participate for non-monetary reasons. This feature has been recognized as central to all communities of 
practice (Wenger et al., 2002), as well as to other peer production processes (Benkler, 2002).

The voluntary nature of participation influences nearly every aspect of the practice of the community. It suggests a shared responsibility for the community's ongoing sustainability, perhaps at least partially explaining why the joint enterprise often includes an aspect of welcoming newcomers. It suggests the importance of common goals, social norms, and persuasion as motivators in place of authoritative directives or cash incentives. It heightens gratitude for other members’ assistance.

Because there is no boss assuring that team members contribute equally, individuals participate however much they desire. Despite the lack of coordination, there is a surprisingly regular empirical observation that online community members' contributions follow a power law distribution, with a core group of members contributing the majority of content and the vast majority of the individuals contributing only marginally (Galegher, Sproull, \& Kiesler, 1998; Lakhani \& von Hippel, 2003; M. Smith, 1999). In fact, there is evidence that most members of online support communities are lurkers with a higher percentage of lurkers in technical support communities than medical ones (Nonnecke \& Preece, 2000). This variation in contributions has been observed in many communities of practice and Wenger, et al. suggest that it is not something that should be changed (2002, pp 55-58). Rather, it is an empirical regularity that should be accepted and accounted for in designs and decisions.

\section{Public or Semi-Public Participation}

Although private online support communities exist, I focus my attention primarily on the vast number of public or semi-public communities. Such communities have fluid boundaries allowing anyone to join or leave at will, although some require potential members to demonstrate their "worthiness" in some, usually trivial way. For example, many communities require members to register with a valid email address in order to 
participate. Such measures are generally taken only to exclude troublemakers, not to limit membership numbers. Public communities allow anyone to read current and archived messages, although only registered members can contribute. Semi-public communities require registration before reading community messages and contributing.

The public nature of online support communities has many implications. It raises privacy considerations, which members may or may not recognize initially, making some hesitant to share personal information. Coupled with the permanence of most threaded conversations it also suggests caution and care when posting a message, a practice that encourages high quality posts (Ackerman \& Palen 1996). It even changes the attitude with which posts are written. Posting messages to the community (and potentially the entire public) is akin to performing on stage, where every action one takes is considered in view of an audience. Some individuals put on a show for the audience with their

eloquent oration, while others lurk in the shadows from stage-freight. Just as a performer cannot recognize everyone in the audience, a contributing member cannot know all of the people who will read their message.

\section{Information Behavior in Online Support Communities}

Information behavior researchers have only recently begun to explore information interactions in online support communities, although they have been studied in other computer-mediated settings since the late 1970s (Hiltz \& Turoff, 1993). While early research on online communities emphasized the social aspects of the interaction, information exchange is clearly a major reason for their existence (Burnett, 2000). Savolainen found that participants of several online communities felt that "discussion groups offered new sources not available in traditional channels. In particular, informal sources could be accessed more easily” (2001, pp 74). Researchers have found that people join online communities primarily to exchange information, rather than for friendship, recreation, a common interest, or social support (Ridings \& Gefen, 2004). 
Online medical support communities were the exception where information exchange and social support were equally important. However, a more recent study of 10 online medical support communities found that information sharing was more prevalent than social support(Meier, Lyons, Frydman, Forlenza, \& Rimer, 2007). It is common for people to initiate contact with communities by asking a question and then sticking around when they believe there are additional gains to be had by continued participation (Sproull \& Patterson, 2004), especially if they receive a decent response to their initial post (Joyce \& Kraut, 2006; Lampe \& Johnston 2005).

Several authors have classified messages in online communities to better understand the primary activities that occur. Most of these have been conducted in medical support communities (see Chapter 8 for a summary). No agreed upon classification scheme is used, so comparisons across studies are difficult, although there have been attempts to create a more uniform classification scheme (Burnett \& Buerkle, 2004). Burnett and Buerkle classified messages into hostile interactive behaviors (e.g., flaming, spamming, trolling), non-informational collaborative behaviors (e.g., humorous, emotional), and informational collaborative behaviors (e.g., announcements, personal updates, queries, responses, group projects) (2004). Future work by the authors will use this typology to compare communities of different types in order to better understand the range of information behaviors in online communities.

Of all of the activities that occur in online communities, those related to information acquisition and use are of particular interest to information behavior researchers. As with other “everyday life information seeking” situations, online communities serve as a source of both practical information seeking and orienting information seeking (Burnett, 2000; R. Savolainen, 1995). Practical information seeking "is aimed at finding specific answers to discrete information needs, often operationalized as specific questions,” while orienting information seeking refers to the monitoring of one’s “information neighborhood” that may be related to ongoing interests and concerns 
(Burnett, 2000). In my own research on css-d I have found that the content experts who stick around are more interested in the information monitoring and social interaction than information seeking, while novices are generally more interested in getting answers to their immediate information needs.

As in other forms of everyday life information seeking, help providers often base their answers on their own experience (Galegher et al., 1998; R. Savolainen, 2001). Not surprisingly, advice provided by several helpers is not always compatible ( $\mathrm{R}$. Savolainen, 2001). Savolainen found that expert organizations as well as secondary sources and hearsay are commonly referenced, while printed materials and friends and colleagues are rarely referenced (R. Savolainen, 2001). While this is true of some online communities, others have strong norms around referencing expert content such as the ones summarized by Wikgren who found that $60 \%$ of all references in health groups refernced sources with scientific medical content. Furthermore, when inaccurate or unsubstantiated claims are made they are often peer reviewed by other community members (Galegher et al., 1998), and are not acted upon without an assessment of their credibility, potential risks, and applicability to the situation at hand (Hansen, 2003). In essence, information gleaned from online communities is typically treated as an “informal” source rather than a formal, authoritative one, although some communities frequently refer to formal sources.

Some research has explored the effectiveness of the question and answering activities that are so prevalent in online communities. Despite the fact that online communities are touted as "interactive," Savolainen found that there was not a significant amount of back-and-forth discussion in order to refine an information need or provide feedback on the usefulness of advice provided, although his study only explored one online community (2001). However, receiving feedback on the usefulness of an information encounter is not commonly done in other information settings such as a 
reference librarian interview, making it difficult to determine the amount of interaction necessary to help satisfy an information need.

Because of the haphazard way we seek for information and refine our needs, even if the answer to a question does not completely fulfill a need it is often still useful to the recipient. For example, Lakharni \& von Hippel found that $17 \%$ of all messages were completely answered, $46 \%$ were partially answered, and 39\% were not publicly answered (although they estimate that $40 \%$ of the publicly unanswered questions were answered in private) (2003). Although it is hard to accurately measure the effectiveness of questions and answers in online communities, the fact that they are so widely used for information seeking is indication that a significant number of people derive value from them. However, there seems to be considerable room for improvement.

\section{Differences among Online Support Communities}

Our definition of online support communities has left room for plenty of variation, even among communities that share the common characteristics discussed above. This section is intended to draw attention to some of the most important differences among online support communities. Potential designs, suggested policies, and other recommendations should be considered in light of these key differences. Below is a list of what I believe are the most important considerations based upon empirical studies of online support communities and communities of practice in general and my own experience with online support communities.

\section{Support Topic}

In defining online support communities I have argued that the joint enterprise of the community must be related to the provision of support for those who are trying to make sense of, and deal with, a particular situation or knowledge domain (i.e., support topic). The support topic of the community largely determines who joins the community, 
the ways in which they communicate (i.e., their shared communicative genres), the resources they call upon, what is acceptable to talk about, and even the tone of the conversation. For example, Preece has found stark differences in the amount of empathetic communication between different types of online support communities (1999b).

In Taylor's vernacular, the support topic will largely determine the "information use environment” (1968). As such, it will determine what people are drawn to the community and their personal characteristics, the types of problems that are addressed, the availability of resources, and what information is valuable in resolving problems (Taylor, 1968). For example, a technical support community may attract engineers with high levels of education, consider well structured yet complex problems with agreed upon assumptions, have access to numerous online information resources that are quickly outdated, and primarily be concerned with instrumental knowledge (e.g., know-how). In contrast, an online support group for recovering alcoholics may attract a more diverse group with varying levels of education, deal with unfamiliar and ill-structured problems, have access to online resources that are relatively consistent over time, and be concerned primarily with personal and motivational knowledge.

\section{Community Size}

Online support communities can range from a handful of people to thousands. The size of the community is important in establishing expectations of participation, determining what community roles are useful, what activities are possible, and what technologies are appropriate. Preece argues that online communities must not be too small to generate sufficient participation and attract new participants or too large to keep an intimate feeling (2000, pp. 91-92). She sees enhanced visualization techniques as a technology that helps larger communities retain some of the intimacy of a smaller group (Preece, 2000, pp. 92-94). Butler, et al. point out that certain types of activities (e.g., 
posting a message) are unaffected by the size of the group, while others (e.g., controlling deviant behavior) are more difficult as group size increases (2002). Constant et al. found that the amount of both good and bad advice increase with size (Constant, Kiesler, \& Sproull, 1996). Wenger, et al. see size as a differentiating characteristic of communities of practice in general and argue that different organizational structures are needed to allow for all participants to feel sufficiently involved (2002, pp. 24-25).

\section{Membership Duration}

There is generally wide variation in the length of time that individuals participate in any given online support community, due to the large variation in the individual needs of members. Butler recorded an annual dropout rate of $22 \%$ from his large sample of online communities (Butler, 1999), although it was more than offset by new members. Despite the individual variation, the support topic will influence the typical length of stay. For example, online support communities related to a chronic medical condition or technical support communities related to an enduring issue (i.e., website design) are likely to have many long-term members. Communities related to more short term needs, such as a support group for new hires at a large company will, by their very nature, not encourage continued participation. Membership duration can affect the sustainability of a community (Butler, 2001), the cohesion of the group, and the importance of embedding knowledge in routines and technology rather than individuals (Argote \& Darr, 2001).

\section{Member Diversity}

There are many dimensions of diversity, ranging from education to religion to ethnicity. There will inevitably be some diversity among members on some of these dimensions. However, the level of diversity can differ widely across communities, at least on the dimensions that are most relevant to the topic at hand. For example, a medical support community could include exclusively patients, or it could include 
patients, doctors, and specialists. Wenger, et al. recognize the heterogeneity of membership as a key differentiator of communities of practice more generally. They argue that "it is often easier to start a community among people with similar backgrounds, but having a problem in common is also a strong motivation for building a shared practice, even among people who share little else” (2002, pp 25-26).

The diversity of a community will affect how much "common ground" there is among participants and thus, the amount and type of grounding that must occur for effective communication to happen (Clark, 1996; Clark \& Brennan, 1991). Grounding is the process through which "we and our addressees [come to] mutually believe that they have understood what we meant well enough for current purposes” (Clark \& Brennan, 1991, p 148). Thus, when there is little common ground due to different backgrounds, community members must spend more effort in order to establish common ground. This extra work takes many forms like clarifying misunderstandings, explaining specialized terminology, rephrasing ideas, and developing a shared repertoire. However, the very fact that there is less overlap of knowledge (or common ground) is indicative that there are often insights to be gained from one another that may not be uncovered without such diversity.

\section{Approach to Social Maintenance}

Online support groups vary significantly in the ways that they deal with deviant behavior, encourage participation, and more generally maintain and regulate behavior. Preece has argued that we have entered an age where online communities must support proper etiquette in order to be sustainable (2004).Both technical and social affordances must be considered in order to achieve sustainability (Ackerman \& Palen, 1996)

Perhaps the most obvious way in which online support communities maintain order is through the activities of one or more list "administrators” (see Preece, 2000 for a discussion of the importance of this role). Administrators often oversee both the social 
and technical aspects of the community by keeping the discussion on-topic, enforcing policies, maintaining infrastructure, promoting the community, and resolving conflicts, among other things (Butler et al., 2002). Some administrators rule as dictators who review all posts before they are sent to the group and exclude troublemakers from posting messages. Other administrators take a laissez-faire approach and let the community members work out the kinks on their own. And still others play the role of a nurturing parent by sending out gentle reminders, giving second chances, and providing ongoing encouragement. Unfortunately, not all of these approaches are effective, and they can lead to further conflict within communities (A. Smith, 1999).

Community members maintain social order through other means as well. These include Netiquitte guidelines that describe what proper behavior is in a particular context, role models that newcomers try to emulate, and regular “citizens” who continually provide feedback through sarcastic comments, gentle reminders, and even use of the “silent treatment” (Preece, 2004). Technologies can also aid social maintenance through the use of filters, moderation capabilities, and rating and reward schemes (Preece, 2004). Slashdot, for example, uses a distributed moderation scheme to help identify deviant behavior, as well as publicly recognize outstanding behavior (Lampe \& Resnick, 2004). Other technologies are used in Ackerman et al.’s I-DIAG system including the use of summary nodes that can close off or redirect threads, as well as agents that check for “flames” or spam robots (Ackerman, Swenson, Cotterill, \& DeMaagd, 2003). Although there may always be a gap between what we need to support socially and what we can reasonably facilitate technically (Ackerman, 2000), the combination of technology and social norms can be a powerful force in maintaining social order, even in situations where there is little formal oversight. Much of the "look and feel” of a given online support community will be driven by the established roles, norms, technologies, and policies that support social maintenance. 


\section{Online Community Repositories}

As discussed in the prior section, most online support communities rely upon some form of the threaded conversation design. Despite its widespread use and advantages, threaded conversation is not well adapted to certain activities that may be of central importance to online support communities. Several communities have recognized this and have supplemented their existing threaded conversation by using various forms of community repositories. These repositories take on many forms from a simple archive of prior threaded conversation to an FAQ document to a collaboratively co-authored knowledge repository. This section reviews the potential benefits, concerns, and challenges of implementing a community repository in a large group setting. But before delving into those issues, I will define what is meant by the term "community repository" and discuss its various instantiations.

\section{Online Community Repository Definition and Sub-types}

In defining the term “community repository” it is important to differentiate it from other related concepts such as "organizational memory" and "knowledge repository." Organizational memory has been a powerful metaphor used for several decades to describe how organizations collectively store, maintain, and retrieve information. It was largely linked to the view that organizations "functionally resemble informationprocessing systems that process information from the environment” and the organizational memory acted as a set of collection bins for the entire organization (Walsh \& Ungson, 1991, p 60). Many believed that information technology could play a significant role in facilitating organizational memory, largely through the use of “knowledge repositories” or “organizational memory information systems” (e.g., Ackerman \& Malone, 1990; Stein \& Zwass, 1995 is typical). More recently, the concept of organizational memory and the ways that technology can best support it have been 
reconsidered. For example, Ackerman and Halverson apply a distributed cognition framework to describe how organizational memory exists, not in a group of knowledge bins, but in a "network of artifacts and people, of memory, and of processing, all of which are bound by social arrangements”(2000). Although researchers understand organizational memory differently, most believe that information technology can play an important role in developing and sharing knowledge within organizations.

An "online community repository" is most closely related to the concept of a "knowledge repository," although in a different setting. It is intended to serve an online community rather than an organization, and as such has significantly different requirements. It consists of recorded information that can be accessed by the entire community (and often the general public). In this dissertation I will restrict the discussion to textual repositories, but other forms of repositories (e.g., auditory) are clearly possible. The repository's purposes may be many, but one central purpose is to retain prior community experiences in a way that will be useful to current and future members. It does not constitute the entire "community memory" since it does not include all of what is in people's heads, relationships, norms, and procedures. However, it is intended to serve the "community memory" by making portions of it more widely explicit and accessible. For example, it may provide answers to potential questions, document community policies, help an individual find an expert, or convey the culture of the group.

\section{Online Community Repository Dimensions}

Online community repositories come in many shapes and sizes, and many communities use more than one type of repository. The two most common types are FAQ documents and discussion archives. Another increasingly popular form of community repository has been prompted by technologies such as wikis that make co-authoring of web documents easier. This has allowed communities (such as css-d) to collaboratively author sets of documents that serve as a community repository. I will call this type of 
repository a wiki repository. Other community repositories have been developed as research projects (e.g., Answer Garden, I-DIAG, Mailinglist Condensation System) (Ackerman \& McDonald 1996; Ackerman et al., 2003; Brewer, 2000). Community repositories differ in how information is contributed, presented, and extracted. A few of the more important dimensions on which they differ are considered next. These are not intended to be a comprehensive design space, rather they are chosen to highlight the most important differences between the FAQs, discussion archives, and wiki repository.

\section{Content Provision}

There are many important decisions about how content is contributed to a community repository. Perhaps the most significant question is, "Who can contribute?" The answer will dependend on how easy it is for individuals to contribute. Traditional FAQ documents were generally maintained by a single author, although they pulled content from the entire community. Other members would suggest changes by contacting the FAQ maintainer, but generally had no easy means of editing the FAQ document directly. In contrast, wiki repositories make it relatively easy for multiple authors to contribute new content or edit existing content. This allows for a greater diversity of content and distribution of work, but it also creates a "tragedy of the commons" problem and amplifies difficulties associated with collaborative authoring (e.g., conflicts, different knowledge representations).

Other important questions include "What types of contribution are possible?” and “How are contributions made?” New content is automatically added to a discussion archive in its original form any time a message is sent to the list. This assures that the archive includes everything, but not necessarily in a useful format. Typically there is no easy way to delete or edit a message in an archive. The result is that an individual must contribute to the discussion in order to contribute to the archive. In order to make the

archive more usable, the discussion itself must change. A few communities recognize this 
and tailor their practices to better support the reuse of the content. For example, they may enforce the use of subject headers that are meaningful not only to the current discussion, but for later use (e.g., discourage subject headers like "Need Help”; include keywords like “Summary”). In contrast, information must be manually added to a wiki repository, but can be edited at any time. Repository pages are distinct from the discussion, although contributors may quote or reference the discussion.

\section{Basic Unit of Contribution}

Another important differentiator is the form of the basic unit of contribution. In other words, what does a single "entry” in the repository look like? The basic unit of contribution in a traditional FAQ document is a question and answer pair. A single message constitutes a discussion archive entry, and a single "page” constitutes an entry in a wiki repository. The question and answer format has been powerful because it forces the author to focus on what is most relevant to the question at hand. However, it is a fairly limiting discursive form and does not fit well in many situations (e.g., multiple questions require the same answer; declarative knowledge is needed). Messages and wiki repository pages are more flexible in their content, allowing for questions and answers as well as other prose. Social norms and some technical constraints generally limit the “acceptable” length of messages to be shorter than acceptable wiki repository pages. In addition, wiki repository pages often support structural elements such as tables and annotations better than discussion messages. Finally, a message is identified by its Subject and Author, whereas a wiki reference page is identified by its title.

\section{$\underline{\text { Navigation }}$}

Navigation deals with how the basic units of contribution are accessed and related to one another. We have suggested elsewhere that there are four main navigational techniques including grouping, sorting, linking, and searching, each of which supports 
different ways of finding content (Hansen, Resnick, \& Riedl, 2005). For example, grouping facilitates browsing of the material that is grouped together. In other words, it is easy to move between items within a single group, but generally more difficult to move across groups. Sorting facilitates selection based upon comparison to other items. Moving between items based upon their similarity or difference become easy. Linking facilitates movement from one message to another even when the messages don't necessarily have an obvious connection. Searching facilitates the retrieval of specific information. Each of these techniques can be based upon a number of factors (e.g., time, content, writer, relationship to artifact, rating).

In practice, several techniques are often used together. A discussion archive is typically searched based upon keywords. However, once a single message is found, it is typically displayed in a group of related messages (i.e., a thread) and sorted in chronological order. Many discussion archives also link to messages by the same author. In contrast, an FAQ document typically sorts the question and answer pairs based upon some measure of importance. Readers are typically forced to browse through them in order, although some allow for searching based upon keyword. Wiki repositories often provide searching based upon keyword, but also facilitate linking between entries (e.g., via hyperlinks). They may also provide an alphabetically sorted index of all pages (based upon the page titles) or grouping through the use of Categories (which work like tags associated with wiki repository pages).

\section{Distillation Support}

The final dimension we will consider is the support that the repository has for summarization and distillation of the conversation. Summarization refers to the presentation of the substance of the conversation in some condensed form (either textual or visual). Distillation refers to the separation or extraction of the essential (or most pure) elements from the conversation. A basic discussion archive does not explicitly support 
any distillation or summarization, although individuals often take it upon themselves to post messages that summarize prior messages. Traditional FAQ documents are intended to be a distillation of only the most "frequently asked questions" along with a summary of the best answers from the discussion. The FAQ genre strongly suggests that the author select and summarize content. Wiki repositories are also typically seen as a distillation of the conversation, or at least the parts that are most important. Contributors can select and summarize prior messages, including ones that are not in a question and answer format. Because they support structures such as tables, they can also be used to aggregate information from the discussion into annotated lists. The acceptability of longer posts also makes it possible to distill larger "chunks" of information than can be dealt with in a single question and answer.

The table below compares the three types of community repositories discussed in this section with one another based upon these key dimensions.

Table 2.1: Online Community Repository Comparison

\begin{tabular}{|l|l|l|l|l|}
\hline Repository Types & $\begin{array}{l}\text { Content } \\
\text { Provision }\end{array}$ & $\begin{array}{l}\text { Basic Unit of } \\
\text { Contribution }\end{array}$ & Navigation & $\begin{array}{l}\text { Distillation } \\
\text { Support }\end{array}$ \\
\hline Traditional FAQ & $\begin{array}{l}\text { One or few } \\
\text { authors; not } \\
\text { easily editable }\end{array}$ & Q\&A Pair & $\begin{array}{l}\text { Sort by Question; } \\
\text { Keyword Search }\end{array}$ & $\begin{array}{l}\text { Selection; } \\
\text { Summarization }\end{array}$ \\
\hline Discussion Archive & $\begin{array}{l}\text { Multiple authors; } \\
\text { not editable }\end{array}$ & Single Message & $\begin{array}{l}\text { Keyword Search; } \\
\text { Group by Thread; } \\
\text { Sort by Time }\end{array}$ & None \\
\hline Wiki Repository & $\begin{array}{l}\text { Multiple authors; } \\
\text { easily editable }\end{array}$ & Single Page & $\begin{array}{l}\text { Link by Hypertext; } \\
\text { Alphabetically } \\
\text { Sorted Index; } \\
\text { Keyword Search; } \\
\text { Group by Category }\end{array}$ & $\begin{array}{l}\text { Selection; } \\
\text { Summarization; } \\
\text { Aggregation }\end{array}$ \\
\hline
\end{tabular}

Promises \& Limitations of Community Repositories

Most online support communities have recognized the potential benefits of having some form of organizational memory, whether it is a discussion archive, a traditional FAQ, a wiki repository, or all three. The frequent use of these sources by community 
members (and outsiders) is a testament to their value. However, despite their value, discussion archives and traditional FAQs fail to realize many of the potential benefits of community repositories. Perhaps this is why, more recently, several online support communities have turned to wiki repositories. This section will use these examples to highlight some of the potential benefits, as well as limitations of the various forms of community repositories. The discussion is framed by existing literature on organizational memory, communities of practice, and information behavior, as well as my own experience working with online support communities. After this section I will consider the difficulties associated with their effective implementation.

\section{Increased Efficiency}

Perhaps the most obvious potential benefit of having a community repository is the decrease in effort that is required to perform the work of meeting community members’ needs. The general idea is that information can be reused so that it does not need to be recorded afresh. This has implications for both information seekers and information providers. Let us examine these with regard to question answering, a common activity in online support communities of every sort.

The simple story goes something like this. An information seeker needs a question answered and as a result he first consults the community repository. If his question has been answered in the community repository, he applies the answer and goes his merry way, without ever having bothered the community or expressed his ignorance of the topic. If his question has not been previously answered, he raises it with the community, many of whom may benefit from the interaction since it is covering new ground. In such a case, there are potential efficiency gains for both the information seeker (who did not need to wait for a response to his question) and for the community (who did not have to answer a question multiple times). 
Unfortunately, this simple model glosses over many important realities. First, it assumes that the information seeker is able to characterize his question in such a way that he can find the answer in the community repository. There has been consistent evidence from the information seeking literature that developing a question in such a manner is not trivial (see Chapter 7 of Vickery \& Vickery, 2004 for a summary). For example, Taylor explained four stages of question formation including a visceral need for information, followed by a conscious need, a formalized need, and finally, a compromised need presented to an information system (Taylor, 1968). Moving from one formulation to the next requires work. Moving to the final compromised search query requires knowledge about the subject and the system itself. "If the enquirer's understanding of the public knowledge in question is limited, the expression of information want is likely to be imperfect” (Vickery \& Vickery, 2004, p 180).

There is also evidence that information seekers can benefit significantly from “information intermediaries” who can diagnose problems, help reformulate stated goals, and identify appropriate resources (Ehrlich \& Cash, 1999; Nardi \& O'Day, 1999). Although reading through the repository may help the information seeker refine his question or orient himself better within the information environment (Bates, 1989; Chang \& Rice, 1993), it may not be possible for him to identify or diagnose his information need in a way that will retrieve the appropriate resources from a repository. In summary, the overall efficiency of the interaction may be increased by a short dialogue between the information seeker who has an imperfect understanding of his own information need and a knowledgeable community member who can help diagnose the underlying information need and provide useful resources or answers without much effort.

Second, this simple model assumes that the repository includes information that is in a format that is useful to the information seeker. This will largely depend upon the type of question and the form that the repository takes, but it will clearly not always be the case. Many questions raised in online support communities are highly contextual and the 
answers must ultimately be tailored to the individual's situation. For example, a common information need of many individuals of css-d is the need for a three column page layout that meets a particular set of conditions (e.g., works in Firefox and IE6, must have footer that doesn't break when using frames, can't use exact positioning). While the need for a three column layout is a common one that would return hundreds of hits if searched for in the css-d email archives, each of the times it is raised is unique in the particulars of what is needed and may not be helpful at all to another individual. Ackerman and Halverson have drawn attention to the complex process of decontextualization and recontextualization that must occur for an information "object" to be turned into an information "process" that is useful for both creator and reader (2000). "To properly serve the reader or reuser of the memory, the creator must properly project the consequences of the memory's later use. This can be a difficult matter, although people do it everyday in their work” (2000, p 59). In summary, simply including archived messages in a repository underestimates the work necessary to make information useful in future situations by other individuals, and even when such work is accomplished (e.g., as in FAQ documents) further discussion is often needed to help apply it to the current situation.

Finally, it is important to recognize that increased efficiency may be at odds with the community's joint enterprise. For example, Preece believes that increased efficiency (gained through the use of an FAQ document) would in fact be detrimental to online medical support communities (1999). She observed that community members did not mind repeating information because their participation was not about swapping hard information, it was about "identifying and communicating with others experiencing similar problems” (Preece, 1999, p 65). Recognizing that another member is dealing with the same issue is far more important and relevant to the joint practice of the group than reducing traffic. Although a traditional FAQ focused on "hard information" would likely result in Preece's anticipated effect, a more flexible wiki repository format with joint authorship may be able to capture some of the "soft information" such as accounts of 
patients' experiences and knowledge about who has dealt with similar issues. After all, as Ellis, et al. observed, the background detail that is expressed through repetitive, emphatic exchanges is "just as much information as hard facts, more difficult to come by in traditional ways” (2004, p 151). In fact, Kimble, et al. argued that the process of creating a shared artifact by a work-related community of practice acted as a "catalyst in the sharing of soft knowledge even in the distributed environment” (2001, p 232).

Although information efficiency gains may not be as high as initially hoped for, there are clearly opportunities for support communities to improve their efficiency through the use of repositories. For example, Procter, et al. found that the use of an FAQ document was significantly more convenient and at least as effective at resolving users needs than a telephone interview with a librarian, although not as useful as a face-to-face consultation (1998). However, for the efficiency gains to be realized, without disrupting the pursuit of other community goals, a community must understand the realities discussed above and the specific joint enterprise of the community in question. Speaking of communities of practice more generally, Wenger, et al, summarize this approach in the following manner, “A community must have a shared understanding of what aspects of its domain are codifiable and which are not, and what to do in each case. Successful practice development depends on a balance between joint activities, in which members explore ideas together, and the production of “things” like documents or tools...Documentation is not a goal in itself, but an integral part of the life of the community” (2002, p 39).

\section{Higher Quality Content}

The problem of monitoring and assessing the quality of content in online communities has been a challenging one. Some communities rely on moderation (i.e., review of messages sent to the list), but higher quality may be traded in for diversity, timeliness, and spontaneity. In non-moderated communities the quality of the 
conversation is largely dependent upon who happens to be listening at the moment, rather than the person who is most informed on the subject. Fortunately, errors or inferior suggestions are often caught by the informal peer review of the many members "listening in” on a thread. Unfortunately, these corrections may come too late in real-time or may be missed when searched in a conversation archive (Galegher et al., 1998).

One of the appeals of a community repository is that it can serve as a collection of the highest quality content. Neus describes the impact of a community repository on quality in this way: “By providing a focal point for the community’s knowledge on given subjects, people can stop reinventing the wheel and instead focus on creating the best wheel for everyone. FAQs of active communities are typically of a much higher quality than i.e. Textbooks, simply because there are so many more eyes for scrutiny and the combined know-how of the community helps to polish the text over time, instead of having just a few authors write a text that only gets revised every other year at the most” (2001).

For this to work, repository contributors (or collectors) must be able to create (or recognize) high quality content. If they fail, the result will be that the "authoritative" version is inaccurate making it more likely that people will act upon the faulty information. However, as Neus points out, there are "more eyes for scrutiny" to catch and even fix inaccuracies if the repository is widely used and easily modifiable (2001).

Site for Meaningful Participation

The development of a community repository can give added focus to a community and encourages new forms of participation. Wenger, et al. describe how a community of practice formed by engineers at DaimlerChrysler used the creation of a repository of procedures and best practices as an opportunity to develop the community's practice more fully. In this case, the engineers found that participating in the creation of the repository was “just as important to them as having the final documents” (Wenger et 
al., 2002, p 39). Kimble, et al. had a similar finding with a distributed community of practice (2001). In summarizing their findings they state "the most striking finding of the case study was the importance of a shared artifact to the community and the range of uses to which it was put” (2001, p 231). Further echoing Wenger, et al.'s statement quoted above, Kimble et al. state that "it is not the artifact per se which is important but the process involved in its creation...[The community members] were able to share knowledge by both participating in the process of creating it, and by participating in the discussions and collaborations which resulted from it” (Kimble et al., 2001, p. 231).

It is important to recognize that for these new forms of participation to occur, the repository cannot simply be an archive of the existing conversation or the work of one community member (as are many traditional FAQ documents). It is through the debates about content, the visible display of expertise, and the friendships that develop through collaboration that the community is enhanced. These can only occur if the process of creating a shared repository is collaborative by its very nature. When it is a collaborative experience, "the twin goals of interacting with peers and creating knowledge products complement each other. On the one hand, the goal of documenting and codifying focuses community activities, and on the other hand, these activities give life and legitimacy to the documentation” (Wenger et al., 2002, p 40).

The potential downside of including new forms of participation through coauthoring of repositories is that they will divert resources (i.e., individuals’ energy and attention) from other important community activities. Wenger, et al. suggest that many communities of practice have failed because they have expended too much energy working on documentation at too early of a stage, when they should have been focused on finding common ground (2002).

Helpful for Newcomers 
Community repositories have the potential to help newcomers in a number of ways. They can help a potential member get a "feel” for the community’s joint enterprise, and possibly even meet some of the core members. This will help potential members determine if it is worth their time to join the community. A community repository can also help newcomers move into a more central role within the community. For example, a repository may help newcomers learn the community’s shared repertoire and typical forms of engagement more efficiently and effectively than they could through observation. Reading of prior discussion archives can provide some of the shared history of the community and reading the community policies in the FAQ may help newcomers understand some of the acceptable forms of engagement. Wiki repositories may even enable "legitimate, peripheral participation" thus enabling newcomers to feel part of the community and transition into a more central community role (Lave \& Wenger, 1991). For example, several members at css-d who are lurkers on the email list have proclaimed themselves "spam hunters" on the wiki repository (see Chapters 5 and 6 for details).

Although community repositories can help attract new members, they can also deter members from joining, even when joining would be in the best interest of the individual and community. Because of the difficulty of creating and maintaining a useful wiki repository, many attempts fail. In many cases, the failed repository is left "out in the open” as a constant reminder of the community’s failure, suggesting to potential newcomers that the community is a stagnant, unmotivated group.

\section{Helpful for Outsiders}

Community repositories are rarely used only by the community that prompted their creation. Online communities often fill a unique niche in the information landscape, sharing information that is difficult to come by in books, manuals, co-workers, and other information resources. Archived messages and FAQ documents from online support communities are often read by non-community members, because they contain 
information that was developed based upon the real-world needs of individuals in a similar situation. The power of an FAQ document derives primarily from the fact that the included topics are determined based upon frequency, and thus are highly relevant to the masses (Neus, 2001). Archived community messages often show up in the results of search engines, suggesting their widespread availability and potential usefulness to noncommunity members.

Unfortunately, it is often difficult for a non-community member to pick up on the often subtle community dynamics at play. For example, when they read a thread pulled from a community archive, they have no idea if the authors are respected community members or individuals that the rest of the community has learned to ignore. They don't know if the suggestions are outdated. They also don't know if the answer to a question is only relevant to the unique situation of the community member who originally posed the question - a situation that did not need to be spelled out because it is part of the community’s common knowledge. These problems can, at least partially, be overcome by intentionally building repositories (like FAQ documents) that are intended for use by individuals who don't yet know all of the community dynamics.

When community repositories serve those outside of the original community that created them, they have the potential to serve as boundary objects (Star \& Griesemer, 1989; Wenger, 1998). Such repositories allow individuals from multiple communities to attach different meanings to their content, while retaining the same structure. In such cases, the original context of the repository's creation and use does not need to be fully understood for others to benefit from the information. An example from my research is the IE7 (Internet Explore 7) wiki repository page created by the css-d community. The community initially created the page as a place to collect information on how effectively the new beta release of IE7 supported CSS (a website design language). Their primary purpose in doing this was to better understand how they will need to use CSS when designing websites in order to make them compliant with IE7 (e.g., what prior IE browser 
hacks will break down or still be needed? What CSS elements are supported?). However, the list moderator recognized that the information would also be useful to Microsoft's IE7 development team as a resource that identified shortcomings in the current version. Indeed, the Microsoft team ended up referring to the page for just such reasons. Although some community repositories (or sections of repositories) already serve as boundary objects, little conscious attention has been given to supporting them as such.

\section{The Challenges of Developing Community Repositories}

Although I have presented a less than utopian view of community repositories, it is clear from many successful examples that they can play an important role in serving the communities that create them and those interested in their content. The potential benefits are especially true of community repositories that are intentionally and collaboratively developed (i.e., wiki repositories), as suggested in the prior section. Unfortunately, wiki repositories can be difficult to successfully design and implement. Below I discuss some of the primary challenges in developing wiki repositories. I also present some recognized strategies for overcoming these challenges. This section is in the same spirit of Marshall, et al. (1994) whose discussion of challenges and solutions is at the level of design principles rather than specific designs.

\section{Initial Adoption of Community Repository}

Before individuals can contribute to a repository, the repository must exist in the first place. This raises one of the most difficult challenges to implementing a community repository, that of obtaining participation from a critical mass of participants (Markus, 1987). This problem can be thought of in terms of a virtuous or vicious cycle. When there is good content in a repository, it will be used by more and more people and will thus attract more attention from potential contributors, who will in turn improve on the content and want to be associated with it. However, when there is poor content, few people use 
the repository and they may not want their names associated with it, suggesting that the content will remain poor indefinitely. Thus, it is necessary to create a critical mass of high quality content and contributors to get a repository jump-started.

One strategy for overcoming the critical mass challenge is to seed the repository with high quality content from the start (Marshall et al., 1994). For example, some of the well respected members at css-d contributed content to the wiki repository before rolling it out to the community, and then to the general public. This helped set a standard for high quality content, as well as make the resource useful from the start, which in turn encouraged future participation from users and individuals who wanted to be associated with it. Other communities seed repositories by selecting exceptional posts from the message archive or from related non-community sources (see Chapters 7 and 9 for details).

Even with a critical mass of content and participants, other problems may hinder the successful adoption of a community repository. Orlikowski pointed out how the introduction of a new corporate organizational memory system failed in part because it threatened existing political structures (1993). Although employee competition is not a core issue in many online community settings, it is important to recognize that the implementation of a wiki repository will likely change the power relationships. For example, in several of the communities I worked with the wiki gave voice to people who felt more comfortable using a wiki than an email list, while marginalizing the views of those who were not comfortable using the wiki (see Chapter 9 for details). Such social changes may lead to a community fracturing into multiple communities or hinder the continued use of the repository by key community members. These possibilities underscore the importance of receiving some sort of buy-in from community members from the start.

Finally, usability issues may hinder the adoption of certain types of community repositories. Designing a repository that can be easily used by the majority of members 
may be an obvious need, but it can be one of the most important issues and most difficult to pull off. This issue becomes even more important in communities where the joint enterprise of the community is not technical in nature (e.g., medical support groups), and thus a high technical expertise of members cannot be assumed. Using technology that is already familiar to users can help alleviate usability issues. Other specific usability guidelines and techniques for collecting feedback from users can be found in various texts (e.g., Nielsen, 1993; Preece, 2000).

\section{Encouraging Contributions}

Even after its initial adoption, it can be a challenge to encourage community members to contribute content to a repository. Contributing to an online community discussion has been likened to the "tragedy of the commons" and the related "free rider" problem (Kollock \& Smith, 1996). In such situations, members benefit from a public good (e.g., others’ comments) but don’t have sufficient reason to “optimally” contribute themselves. Social psychologists use a related concept of "social loafing” to describe the empirical finding that individuals tend to "slack off” when contributing to a group tasks. In their review of social loafing studies, Karau and Williams provide a framework for understanding this phenomenon called the "collective effort model” (1993). They argue that individuals will contribute to collective tasks "only to the degree that they expect their efforts to be instrumental in obtaining valued outcomes” (Karau \& Williams, 1993). In order to fully realize the potential of online communities it is important to develop social and technical interventions that encourage a healthy level of participation (Ling et al., 2005).

So, how do online communities overcome the "free rider” problem? The answer is not an easy one. Researchers have found a wide variety of reasons that members contribute to the community conversation including commitment to a larger cause, reputation gains, reciprocity, learning benefits, expression of self “efficacy,” and empathy 
(Constant et al., 1996; Kollock, 1999; Lakhani \& von Hippel, 2003; Wasko \& Faraj, 2000). Two important themes can be taken away from prior work on contributions to online community discussions. First, there are a wide variety of motivations at both the individual and group (i.e., online community) level. And second, self-interested and altruistic motivations are closely linked and often difficult to separate, even for the individual who experiences them.

Many of the same factors that encourage participation in the online discussion likely encourage contribution to a wiki repository. However, the work of contributing to a wiki repository is qualitatively different than contributing to an ongoing discussion. It requires selecting, compiling, organizing, summarizing, editing, updating, and decontextualizing, all while imagining how the content will be used by others at a later time (Ackerman \& Halverson, 2000). This work is often viewed as “extra work,” much of which may not be intrinsically satisfying (Csikszentmihalyi, 1990, 1996). Furthermore, the reward for contributing to a repository is often not immediate, and the beneficiary of the "extra work" may not be the contributor, all of which dissuade contribution (Grudin, 1988). In summary, contribution to a wiki repository often requires "extra work" that is not intrinsically satisfying and is of only "potential” benefit to others.

Despite these challenges, examples like Wikipedia suggest that there are ways of motivating individuals to contribute to repositories, even if we do not fully understand them. A few studies have examined contributions of movie ratings to an online database that is used as a recommender system (see Ling et al., 2005 for a summary of several related studies). The studies rely upon field experiments and test various social and technical interventions that were motivated by social psychology theories. They found that emphasizing the uniqueness of an individual's contribution and setting group goals increased participation (Ling et al., 2005), as did expert and peer oversight (Cosley, Frankowski, Kiesler, Terveen, \& Riedl, 2005). However, other hypotheses were not confirmed. For example, individuals did not contribute more to groups they believed to 
be more similar to themselves (Ling et al., 2005). Overall, these studies highlighted the difficulty of developing design interventions that encourage participation, as well as the potential value of using social science theories to motivate novel designs.

In addition to providing extra motivation to participate, another way of encouraging participation is to lower its cost. Several authors have pointed to the significance of facilitating micro-contributions (Benkler, 2002; Sproull \& Patterson, 2004). While I may not be willing to write an essay on a topic, if it is easy enough I may be willing to reword a paragraph for clarity or add a missing section. Shipman and coauthors recommend that designers of community repositories allow for "incremental formalization” (Shipman \& Marshall, 1999; Shipman \& McCall, 1999). They argue that users should be able to slowly increase the level of structure and formalities in their material. The I-DIAG system provides tools that enable "incremental formalization" in a virtual community setting where a discussion is "consolidated" into a more lasting form (Ackerman et al., 2003). Incremental formalization lowers the cost of contributing by requiring less effort up front (e.g., knowledge of an extensive controlled vocabulary) and allowing more structured formalization after-the-fact through micro-contributions.

It is important to remember that although we may need to encourage a healthy amount of participation, we should not make the mistake of forcing equal participation among members (Wenger et al., 2002). The boundaries of communities of practice are fluid and people should be able to move seamlessly from peripheral to active to central roles and back again. Instead of forcing participation, communities should "build benches" for those on the sidelines. The trick is to "allow participants at all levels to feel like full members” (Wenger et al., 2002, p 57). The work-benefit disparity will not appear so stark in such situations and as the topic shifts, different members will feel comfortable taking on a more active role. Preece, et al. have explained how this plays out in online community settings where the majority of community members are often sitting in the bleachers (2004). 


\section{Information Degradation}

As touched upon earlier, many communities are related to a support topic that is constantly in flux, such as website design. In such cases, best practices from a year ago may be discouraged this year and links to outside websites are often broken. Whereas the conversation can quickly change to accommodate the current needs of the community, a repository is meant to be more long-term and thus may require ongoing maintenance to keep up-to-date. Marshall, et al. also point out that internal "inconsistencies and redundant contributions” must be weeded out as the community memory grows and evolves over time (1994). If it is not, then information becomes harder to find and parallel discussions emerge around redundant entries (1994).

To combat the problem of maintenance, Marshall, et al. suggest that communities consciously go through the stages of content seeding to overcome the critical mass issue discussed above, evolutionary growth, and reseeding to update and restructure the content for current use (1994). Other practitioners, such as those at Wikipedia, suggest a constant flow of editing and "refactoring" existing content so that it is more useful and integrated with existing content (see "Wikipedia: Refactoring Talk Pages," n.d.).

\section{Obtaining Useful Information}

Even if a repository contains a great deal of useful information, it remains a challenge to get that information to the individuals that can benefit from it. There are two primary factors that make this such a difficult problem. First, content providers can only guess at the future needs of other individuals, making it difficult for them to provide appropriate meta-data that would aid information seekers. And, even if they have a perfect understanding of the information seeker's needs, it is unlikely that they will use 
the same vocabulary to describe it (Furnas, Landauer, Gomez, \& Dumais, 1987). People even find it cognitively difficult to classify their own office materials for personal use (Malone, 1983). Perhaps this is why entire industries have developed to organize and structure information in a way that is meaningful to large groups of individuals through the use of taxonomies, indexes, controlled vocabularies, and the like. Bates has argued that this type of work does not come naturally to all humans and requires some information science expertise to perform well, a situation that does not bode well for online communities that do not include information professionals (Bates, 1999).

The popularity of tagging systems such as del.ico.us and flickr have suggested that non-information specialists ${ }^{1}$ may be able to provide meaningful meta-data, at least for the performance of some tasks (e.g., knowledge discovery) (Furnas et al., 2006). Although the reasons for tagging community success are still being explored, several factors are likely at play. These include the low cost of creating tags, the incentive to contribute tags for personal as well as social reasons, and the group convergence on tags that occurs through learning in a community setting. At this stage, tagging has only been applied to certain domains (e.g., knowledge discovery and sharing) with success and it is still unclear that it is a good design for other activities such as information retrieval (Furnas et al., 2006).

The second challenge is that many individuals who could gain most from the repository content do not know that they need it, or do not know that it is there to be found (Marshall et al., 1994). Newcomers often don’t know that a repository exists. Even if they do know it is there, they may not know what search terms to use or how to navigate to a relevant section of the repository. Members that do know of the repository's

\footnotetext{
${ }^{1}$ It could be argued that many early adopters of tagging sites are in fact information scientists, or at least are quite "information literate." Only time will tell if tagging will continue to pay off with other communities and in other settings.
} 
existence may not find it worth their time to keep up with all of the information in the repository, especially if it is constantly changing.

One simple strategy that has helped alleviate this second challenge is to make it easy for the community to recommend content (including repository content) to those in need. Marshall et al. state, "Perhaps the central point about obtaining information within communities of practice is that informed people are frequently the best source of information. This function of community as information agency--i.e., as mediator of retrieval--is in fact one of the primary reasons for its existence. Supporting this function is thus decisive for the creation of successful electronic communities of practice" (Marshall et al., 1994). In this sense, an online support community can be thought of as a group of potential information mediators with knowledge of the specific domain. Although it has not yet been applied to online communities, the long history of research on improving the "reference interview" that librarians perform could help inform best practices in this area (Durrance, 1995; Nardi \& O'Day, 1999). An alternate approach is to use automated recommender systems (e.g., Amazon's book recommendations) to help suggest content based upon some information from the user's profile or current activities (Resnick \& Varian, 1997).

It is also important to recognize the potential for repositories to help members find one another. Most community member insights cannot be stored in a repository. However, that does not mean that a community repository cannot help provide access to such information. Marshall, et al. suggest that the repository can help in two ways (1994). First, it can help collect the questions that members want addressed, so that other members can become aware of the information needs. And second, "it can store information about the types of knowledge possessed by its various members - i.e., who knows what types of things” (Marshall et al., 1994). Ackerman and McDonald describe technology that helps to "gracefully escalate" information needs so that they are brought 
to the attention of more and more people with appropriate levels of expertise when necessary (Ackerman \& Mcdonald, 2000).

\section{The Interplay of Community Discussion and Repositories}

In the prior section I have highlighted the existing and potential role of a community repository with regard to online support communities. In doing so, it has become apparent that a community repository is not primarily a replacement for a community discussion. Instead, there is a complex interdependent relationship between the two that has not yet been characterized in the research literature. This dissertation will attempt to characterize this relationship by exploring the activities that help link the resources together, as well as documenting the ways that each resource can benefit from (or be harmed by) their close association.

\section{Conclusion}

In this chapter I have defined online support communities, arguing that they are distributed, computer-mediated communities of practice focused on supporting the needs of individuals who are trying to make sense of, and deal with, a particular situation or knowledge domain. Currently, most of these communities are voluntary, public (or semipublic), and based upon the threaded conversation design. While I have intentionally defined online support communities broadly, it is important to recognize key differentiators and their implications, including support topic, community size, membership duration, membership diversity, and administrative approach.

Many online support communities have added some form of repository to the existing threaded conversation, in the form of a discussion archive, an FAQ document, or a wiki repository (i.e., collaboratively authored web pages). These repositories have the potential to increase efficiency and quality of information, provide new forms of participation, and create useful information for newcomers and outsiders, although there 
are limitations and concerns associated with many of these. Traditional FAQ documents are limited in their ability to capitalize on these potential benefits by their inflexible format and lack of genuine co-authorship. Discussion archives include the original context, but they are difficult to retrieve information from and don’t encourage distillation of knowledge. Wiki repositories show great promise in overcoming the limitations of FAQs and discussion archives, but have significant challenges to overcome, including encouragement of contributions, obtaining critical mass, dealing with information degradation, and making information in them accessible.

Much of the research summarized in this section has been performed in settings other than online support communities. I have attempted to select theoretical constructs and empirical results that are likely to apply to online support communities, but it is likely the case that new opportunities and challenges arise in the online support community setting. It is also true that much of the literature I have drawn upon provides design recommendations at a fairly high level (e.g., facilitate evolutionary growth of a repository). While some of these suggestions have been developed with specific designs in mind (e.g., Marshall et al., 1994), it is not clear how to apply some of them to specific design choices (e.g., Wenger et al., 2002). The remainder of this dissertation helps fill that gap by focusing on design-level choices as they relate to online support communities. It will also help to clarify the relationship between ongoing conversation and knowledge repositories and flesh out the ways that each can benefit from the other. 


\section{CHAPTER 3}

\section{CSS-D STUDY INTRODUCTION \& METHODS}

I argued in Chapter 2 that although wiki repositories are used by many online support communities (especially technical support ones) and have great potential benefits, they have not been well studied empirically. Although the research summarized in Chapter 2 provides some ideas on how to effectively use a wiki repository, it has not been conducted in an online support context and has often been too high level to offer practical suggestions useful for community leaders. The empirical study introduced in this chapter and continued in Chapters 3-7 helps fill this gap by providing a nuanced view of an online technical support community, css-d, that has successfully augmented its threaded conversation with a wiki repository.

In this chapter I introduce the project goals and methods used to meet those goals, as well as introduce the community that was empirically examined. It is broken into the following sections:

- Research Goals

- Rationale for a Case Study Design

- Why css-d?

- Cascading Style Sheets (CSS) \& the css-d Community

- Research Questions

- Sources of Data

- Theoretical Frameworks \& Methodological Stance

- Methodology 


\section{Research Goals}

As discussed in Chapter 2, wiki repositories have the potential to improve online communities in several ways, although they have not been empirically validated in an online support community setting. These include increased efficiency (e.g., by reducing frequently asked questions), improved content quality, new forms of participation, and help for newcomers and outsiders. One of the goals of this study was to empirically validate these potential benefits and determine if wiki repositories offer other unexpected benefits and costs.

Another goal of the study was to understand the challenges of implementing and maintaining a wiki repository, as well as best practices for overcoming those challenges. Some potential challenges were identified in Chapter 2, such as obtaining a critical mass of participants, encouraging contributions, keeping content up-to-date, and helping visitors find relevant information from within the repository. However, these have not been documented in an online support setting and the strategies for overcoming them in such a setting have not been identified.

A final goal of the study was to help formulate general principles of inquiry related to the combination of conversation and documents of more lasting value (i.e., wiki repository pages). What are the strengths and weaknesses of each? What is the nature of their relationship? How can they be coupled together through social norms, community roles, and specific technological features? What work is required to maintain this coupling? In what ways do they draw from each other? This deeper understanding will help lay the groundwork for future research on this topic, as well as help inform potential novel design suggestions.

To meet these goals, I performed an empirical examination of an online support community that had successfully integrated its threaded conversation with a wiki 
repository. The following sections describe why I chose to use a case study design as a basis for this project and why css-d was a good candidate.

\section{Rationale for a Case Study Design}

Researchers interested in better supporting cooperative endeavors (e.g., online communities) have used a wide array of methods. I chose to perform an in-depth empirical study of a single community for several reasons. First, the area of inquiry is new and lacks clear hypotheses that can be tested using other methods such as lab experiments or statistical methods. In other words, it is ripe for an empirical examination that can inform theory development. An empirical examination of a community (i.e., a case study design) is well suited for developing theory "in the early stages of research on a topic," particularly when "little is known about the phenomenon [or] current perspectives seem inadequate because they have little empirical substantiation” (Eisenhardt, 1989). In this project, I explore theoretical issues related to the nature of combining community conversation and collaborative authoring of repository documents as discussed in the Research Goals section above.

The second reason for using a case study design is that the subject of inquiry is an entire system of action, not the typical behaviors of an individual or even the aggregate behaviors of a group of individuals. Feagin, et al. argued that case studies are most appropriate when trying to gain a holistic understanding of a cultural system of action (1991). Case studies help shed light on interrelated activities engaged in by actors in a particular social setting. A case study of an online support community draws the boundaries of inquiry precisely around the thing (i.e., the system of action) that is trying to be understood. It also draws the boundaries around the system of action that community leaders and designers can influence, which is important in meeting the practical goals discussed in the prior section. 
Finally, case studies are amenable to a holistic, grounded approach that can lead to insights that are useful in practice and at a level of analysis that can inform system design. Eisenhardt argued that although case studies run the risk of resulting in overly complex and "narrow and idiosyncratic theory," because they are so close to the data, the resulting theory is likely to be empirically valid, novel, and testable in other settings (because it is based on readily measured constructs) (1989). Other researchers have argued that ethnographic examinations (including case studies) can provide insights about social interaction that are useful for designers of collaborative technologies (e.g. Button \& Dourish, 1996; Hughes, King, Rodden, \& Andersen, 1994).

\section{Why css-d?}

Each case study should clearly define its boundaries by selecting and defining a specific case (Yin, 2003). This study focused on the interrelated system of action engaged in by the css-d community (described later in this chapter). The selection of a specific case (here css-d) is an important aspect of case studies because it "controls extraneous variation and helps to define the limits for generalizing the findings” (Eisenhardt, 1989, p. 537).

Unlike statistical sampling, cases need not be chosen randomly. The generalizing that happens is not from a representative sample to a population, as in statistical sampling. Case studies "are generalizable to theoretical propositions and not to populations or universes” (Yin, 2003, p. 10). Case study sampling should instead be based on theoretical and practical considerations. The specific reasons for choosing css-d are outlined below.

\section{Successful}

Css-d successfully created a wiki repository to augment its threaded conversation, as indicated by the repository's continued use by community and non-community 
members. The continued use of the repository by community and non-community members was the primary evidence for success. Its success was verified by the positive perceptions reported by many community members throughout the project. The success criterion assured that there would be some best practices to glean from the community. Although the community has succeeded at creating and maintaining a useful repository on the whole, the repository is by no means perfect. The case study is intended to learn not only from the successes, but also from the failures of specific practices related to the wiki repository and threaded conversation.

\section{Accessible \& Comprehensive Data}

The community's activities are well documented and accessible for research (see Sources of Data section later in this chapter). Importantly, data were available for time periods before the repository was implemented and throughout its growth. This allowed us to look at best practices related to the implementation of the repository, as well as its continued maintenance over time. Although it was not clear from the outset, we were fortunate that many of the original, active community members were available for interviews.

\section{Representative}

The community clearly fits the definition of online support community outlined in Chapter 2. This is important in that I am interested in identifying best practices that will be potentially useful to other online support communities. Further studies are clearly required to test the transferability of the findings to related communities, but as a first step it was important that the nature of the work was prototypical of the work occurring in online support communities more generally. Some of the factors deemed important were the community's reliance on traditional threaded conversation (via an email list), a wide variation in members' levels of expertise, and a focus on asking and answering 
practical questions related to a specific area of interest. Because of my interest in transferring these findings to medical support communities, I was pleased to find that cssd included examples of strong social bonds and a very welcoming atmosphere for newcomers - characteristics typical of medical support communities (see Chapter 8).

\section{Cascading Style Sheets \& the css-d Community}

\section{Cascading Style Sheets}

Css-d is centered around the specific technology of Cascading Style Sheets (CSS), which is a style sheet computer language used to add style (e.g., fonts and spacing) to structured web documents (e.g., HTML documents or XML applications) (Bos, Çelik, Hickson, \& Lie, 2006). It is intended to simplify the creation and maintenance of websites by separating the styling aspects (controlled by CSS) from the content. To fully understand css-d it is helpful to understand a few characteristics of CSS technology itself.

Like other technologies, CSS is constantly changing, as is its relationship with other web design technologies. The primary drivers of change in this area are revisions to the W3C CSS specifications (Bos et al., 2006), new releases of web browsers, developments of new techniques (i.e., hacks) and best practices, and changes in the preferences and needs of website designers.

Although the CSS specifications are relatively straightforward, in practice the use of CSS can be extremely complex. This complexity derives largely from web browser bugs and inconsistencies in the way browsers interpret the W3C CSS specifications, making it hard to create a page that looks good in every browser. Problems can be difficult to diagnose and there are often numerous possible solutions to a problem, each with its own side effects and complex sets of contingencies. Thus, the best solution to a problem for one person may be completely different than the best solution for another person. 
CSS differs from some technologies because of its visual nature. Like many information technologies, CSS relies upon code to perform some action. Unlike many technologies, CSS code affects the visual display of information including colors, lines, images, fonts, and object sizes. In this context, a screenshot is often worth a thousand words. Thus, it is common for CSS developers to directly link to websites that display a certain problem or effect in order to help others understand the outcome of the CSS code. It is also common for designers to look at their websites in a variety of web browsers, since each one renders a slightly different picture with the same CSS code.

\section{css-d Community}

The css-d community began in January 2002 as a public mailing list devoted to discussions about the applied use of CSS. It was started by a well-known CSS expert and author and included over 1,000 members within the first month. It has continued to grow to over 7,000 members in May of 2006 and includes professional and amateur website developers from a number of countries. It is an active list with over 50 messages sent on average in a day and many more sent privately to list members. Like many online technical support communities, it is an unmoderated list that is "run" by volunteer administrators who maintain the email list software, keep the discussion friendly and ontopic, and remind members of email list policies. The main community website ("Css-d wiki,") describes how to subscribe to the list, includes a description of the list and its policies, and links to the list's archives and wiki.

In August of 2002, eight months after the list's inception, the community created a wiki repository using the WikkiTikkiTavi wiki engine (i.e., the wiki) ("WikkiTikkiTavi,"). Initial content in the form of a network of hyperlinked wiki pages was provided by a handful of members. The wiki has continued to grow over time and currently includes hundreds of pages discussing css-related content, community 
information such as mailing list policies and member biographies, and wiki software help pages.

\section{Research Questions}

Each empirical examination should begin with a research question that focuses everything from the case selection to the data collection to the analysis (Eisenhardt, 1989; Yin, 2003). I have already outlined some of the driving goals of the research project and how they influenced the selection of the css-d community. However, to help focus the data collection and analysis, it was necessary to clearly define research questions specific to the css-d community. Methodologists suggest that "How" and "Why" questions are most appropriate for case study designs (Yin, 2003, p. 21). After initial review of the community, I settled on the following primary research question:

Primary Question: How is activity organized at css-d to leverage both the email list conversation and the wiki repository?

Answering this question requires a holistic look at the activities occurring in both the email list conversation and the wiki repository. It also requires a look at the social norms, technological features, community roles, and perceptions of these activities in order to learn how and why the activities are performed. All of this helps describe how the activity is organized. In addition, it requires a look at the effect of these activities on the quality and effectiveness of the work occurring at css-d. This is what allows us to separate the helpful activities that help leverage both resources from those that are unhelpful or even problematic. The two sub-questions below capture these needs and helped guide the collection and analysis of data throughout the case study.

Sub Question 1: What activities, genres, roles, perceptions, social norms, and technological features are important in coupling the wiki and email list together so as to leverage both resources? 
Sub Question 2: What are the implications of the current arrangements on the quality and effectiveness of the work happening at css-d?

It is important to recognize that these research goals are only a first step in a larger research agenda that characterizes the relationship between community conversation and repositories. As a single case study, it provides a detailed look at one point within a broader design space of possible social and technical arrangements. Later chapters in this dissertation and future research beyond this dissertation will help fill in the design space more completely.

\section{Theoretical Frameworks \& Methodological Stance}

Empirical studies are performed using a variety of theoretical frameworks and accompanying research methods. To a large extent, these determine the shape and validity of the findings. In addition, a clear articulation of them can help readers interpret the findings by illuminating some of the author's basic assumptions.

Eisenhardt suggests that when developing theory from a case study, researchers should begin "as close as possible to the ideal of no theory under consideration and no hypothesis to test” (Eisenhardt, 1989, p. 536). Starting from as clean a slate as possible allows the researcher to ground their theory in the data and not their own preconceived notions. Glaser and Strauss have argued for the benefits of "grounded theory" that is emergent from the data (Glaser \& Strauss, 1967). Namely, they argue that grounded theory is testable, relevant, and valid because of its close connection with the data. As much as possible, I have followed this approach by letting the data drive my inquiry, analysis, and findings. However, the data has clearly been filtered through my own theoretical stances and experiences to some extent. In order to make my own biases more transparent, I describe two frameworks that have influenced my thinking.

\section{Structuration Meta-Theory}


Social scientists have long argued about the importance of several dichotomies including agency/structure, subjective/objective, and micro/macro. Structuration theory, as introduced by Giddens, is an attempt to reconcile these dichotomies by recognizing and giving equal weight to each of the dichotomous terms (1979; 1986). Structuration theory helps articulate the process whereby individuals produce, reproduce, and change social structures, as well as the ways they are influenced by them. The key insight is that there is a "duality of structure” whereby social structures are called upon by individual actors, and in the very act of calling upon them are reproduced and sometimes modified. Giddens uses language as an example of this process which also occurs with social action: “when I utter a grammatical English sentence in a casual conversation, I contribute to the reproduction of the English language as a whole” (Giddens, 1979, p. 77).

Structuration theory has been applied to technology adoption within organizations by several authors (e.g., Pozzebon \& Pinsonneault, 2006 for a list). Orlikowski et al. argue that "the structuring of technologies in use refers to the processes through which users manipulate their technologies to accomplish work, and the ways in which such action draws on and reproduces (or sometimes changes) the particular social contexts within which they work” (1995). Viewing technology use in this way suggests that we examine how individuals use technologies in practice(Orlikowski, 2000). The research focus shifts "from a focus on given technologies, embodied structures, and their influence on use - to a focus on human agency and the enactment of emergent structures in the recurrent use of technologies (Orlikowski, 2000, p. 421). This focus is particularly well suited for studying generic, open-ended technologies (e.g., email, wikis) where a variety of usage contexts can be enacted (Orlikowski et al., 1995).

Throughout this case study I have ascribed to the basic tenets of Structuration theory by focusing attention on the use of technology in practice. This has included both a study of individual actions (and motivations), as well as the ways in which they are 
enabled and constrained by existing social structures (e.g., social norms, roles, and communicative genres). Although I relate these findings to specific technological features, I have not assumed a priori that the features will be used (or are best used) as envisioned by those who created the technologies.

\section{Social Science in the Service of Design}

There has been an ongoing discussion beginning with Simon about the interplay between social science which attempts to understand "the way things are" and design which attempts to determine "how things ought to be" (Simon, 1996, p. 114). Recently, much of this discussion has focused on the relationship between ethnography and design. Several authors have argued that ethnography performed as it was originally intended (e.g., in ethnomethodological studies) is not well equipped for informing design (Button \& Dourish, 1996; Shapiro, 1994). These researchers have argued for hybrid approaches that utilize familiar methods (e.g., ethnography) but with an eye towards design. Here, and throughout this dissertation, I use the word design in the broad sense suggested by Simon (1996). It includes not only technological designs and feature, but also social practices enacted with a particular result in mind.

I take a stance similar to the one taken by the authors quoted above. The research goals and questions that I have developed relate very closely with design in the broad sense. Thus, at times I have focused in on specific technical features or social actions because they are pertinent to design (i.e., they have the potential to influence the way things ought to be), not solely because of their explanatory power. In doing this I have tried to be faithful to the larger social processes at play, but my instinct to look for social and technical design choices and levers cannot be ignored.

\section{Sources of Data}

The following data sources were used for the css-d case study. 


\section{Email List Messages}

Email messages were available from the list's inception in January 2002 to the present time through the list’s public email archive (January 2007) ("Css-d Public Archives,"). In total this includes close to 90,000 messages. Unfortunately, messages in the public archive do not include all of the email messages sent to the list due to some glitches with the custom program used to display them. Furthermore, the public archive hides email addresses, which can be helpful in determining unique posters. For these reasons, much of the analysis is based on a subset of messages that were available in their original form through the private mailing list archive (available via css-d website "Css-d wiki,"). This subset included all 45,580 messages sent between January 2003 and April 2005. The content of each message and header information (sender, subject, timestamp) was available. Certain messages were over-sampled including those that reference the wiki or email list, as well as those that include "ADMIN" in the subject line.

\section{Wiki Content}

In August of 2002 (eight months after the list was created) a member set up a web-based collaborative authoring tool based upon the WikkiTikkiTavi wiki engine with the support of the list administrators ("Css-d wiki," n.d.). The contents of all wiki pages were downloaded locally on January 8, 2007 for analysis. The wiki stores all prior versions of a page, but they are only stored for a couple of weeks, so it was not possible to access the entire history of the wiki. We were able to capture several snapshots of earlier versions of the wiki taken between April 2003 and May 2006 that were recorded in the Internet Archive or by the authors. In addition to the content of the pages, the size (as measured in Bytes) of each page was available through a special wiki page that was generated for our different snapshots. Figure 5.1 shows the wiki size (in KB) and number of pages for each of the snapshots in time for which I had data. 
The wiki includes a page called the RecentChanges page (see Figure 5.6), which displays the title of each page that was recently edited, along with the editor's username (if logged in) or IP address (if not logged in). It also includes a short free-text description of the edit, if one is provided by the author. The RecentChanges page was captured along with the other pages for all of the snapshots.

\section{Wiki \& Public Archive Server Log Reports}

The company that hosts the wiki, Incutio, gave us access to reports summarizing the server log data of the wiki and public archive sites. The wiki site reports were available beginning May 3, 2004, while the public archive reports were available beginning September 25, 2004. The reports were created by the Advanced Web Statistics 6.5 software package (Destailleur). The variables of most interest in this study included the number of unique visitors (excluding robots and spiders), total hits, visit duration, prior origin of visitor (e.g., search engine, other website, direct access), and search terms that led visitors to the site. Specific page visit data was not available in the wiki reports, but was available in the archive reports (where each individual message has its own unique web page URL).

\section{Interviews}

Over a 12-month period, I conducted a total of 14 semi-structured phone interviews (between a half-hour to an hour long each). Interviewed members included all 4 current or past list administrators, all of whom regularly post to the list and wiki, 7 participants who regularly posted to the email list and wiki, two members who regularly contribute to the wiki but not to the email list, and one participant who contributes to the email list but not the wiki. In addition, I interviewed 5 members via email and received

voluntarily provided comments from 4 others. Table 3.1 summarizes the interviews based upon participation patterns. 
Table 3.1: css-d Interview Sample

\begin{tabular}{|l|l|l|l|l|l|}
\hline & Admins & $\begin{array}{l}\text { Actively } \\
\text { Contribute to the } \\
\text { List \& Wiki }\end{array}$ & $\begin{array}{l}\text { Primarily } \\
\text { Contribute to } \\
\text { the Wiki }\end{array}$ & $\begin{array}{l}\text { Primarily } \\
\text { Contribute to } \\
\text { the List }\end{array}$ & $\begin{array}{l}\text { Rarely } \\
\text { Contribute to } \\
\text { the List or } \\
\text { Wiki }\end{array}$ \\
\hline $\begin{array}{l}\text { Phone } \\
\text { Interviews }\end{array}$ & 4 & 7 & 2 & 1 & 0 \\
\hline $\begin{array}{l}\text { Email } \\
\text { Interviews }\end{array}$ & 0 & 1 & 1 & 2 & 1 \\
\hline $\begin{array}{l}\text { Email } \\
\text { Comments }\end{array}$ & 0 & 0 & 0 & 1 & 3 \\
\hline
\end{tabular}

The group of interviewees is not representative of the overall population. Like other online support groups, participation is highly skewed in both the email list and wiki with a handful of individuals providing the vast majority of the content. This sample includes several of the active contributors to both the wiki and email list, however it is lacking in its coverage of the much larger group that consists of lurkers on the email list and readers of the wiki content who never post to it. As a result, the interviewee sample was able to provide insights concerning the creation of a large portion of the email list and wiki content. However, it was less helpful in understanding how that content was interpreted by novices and infrequent posters who make up the majority of the members.

Interview questions asked about several items including:

- the nature of css-related work,

- css resources (e.g., websites, people, books), including how the email list and wiki compared to those other resources,

- the expertise of the individual,

- experiences with other email lists and wikis and how they differ from this one,

- the primary activities and genres that occur on the email list and wiki,

- their motivations for contributing,

- unobservable behaviors (e.g., emailing messages outside of the list),

- the relationship between the email list and wiki, and

- perceptions of the email list and wiki. 
In addition, interviewees were asked why they performed certain actions of interest (e.g., "why have you referred others to the OffTopic wiki page in email messages?”; “what prompted you to edit the IE7 page?”).

\section{Data Analysis}

I used a mixed method approach in order to gain as comprehensive and detailed a view of the css-d community as possible, as well as to strengthen the validity of my findings. This approach is recommended for empirical case study research (Eisenhardt, 1989; Yin, 2003). The overall research process was highly iterative where data collection, analysis, and theory development all overlapped. This overlap allowed preliminary hypotheses to be developed, refined, or dropped as further evidence came to bear on it. It also helped guide future data collection (Eisenhardt, 1989; Glaser \& Strauss, 1967). This allowed me to explore certain sub-topics within the realm of the research question that I had not originally anticipated. For example, the importance of the wiki in maintaining social order within the email list was an important emergent area of exploration. I continued the iterative investigative practice until I believed that the major themes were well enough understood and the major claims supported. The following sections describe some of the specific analyses performed and how they complement one another.

\section{Quantitative Analysis of Participation}

I began by analyzing email list messages to understand overall participation patterns. This was performed using Mailbag Assistant software, which manages large numbers of email messages and allows scripts (e.g., regular expression search strings) to be run on them ("Mailbag Assistant 3.82,"). It also allows messages (or header information) to be exported in formats amenable to other software analysis programs.

The quantitative analysis provided some basic statistics related to the frequency of posts, length of threads, and helped identify individuals at varying levels of participation 
over time. ${ }^{2}$ It also helped get an idea of the tempo of activity including how quickly messages are replied to and how long members participate in the email list. All messages that reference the wiki were analyzed to see who referenced the wiki, and which pages were most often referenced. This initial quantitative analysis helped identify unique social roles within the community and suggested who should be interviewed. For example, frequent posters who referenced the wiki often were separated from frequent posters who did not reference the wiki, who were further separated from those who referenced the wiki often but did not post frequently to the list. Furthermore, those who primarily asked questions were separated from those who primarily answered questions and those who frequently did both.

I also analyzed the wiki pages. Using various snapshots in time I was able to calculate the number of new pages, deleted pages, and growth of pages (as measured in Bytes) over time (see Table 5.1 for a summary of total pages and size). I was also able to see the overall distribution of page size.

\section{Content Analysis}

In parallel with the quantitative data analysis, I performed a content analysis of the email list messages and wiki pages using a grounded theory approach (Glaser \& Strauss, 1967). The goal was to understand which activities occurred in the wiki and email list, their similarities and differences, and their interactions. I also wanted to

${ }^{2}$ In order to accurately identify unique posters I tried to identify (when possible) individuals who used different names or email addresses but referred to the same person. To do this, I counted people as the same individual if they listed different (e.g., "Johnathan Smith" and “John Smith", or even "CSS Master"), but the same email address. I also counted individuals with the same name but multiple addresses as the same individual. This process resulted in the reduction of the original list of 5,000 unique "From:” strings to 3,894 unique "individuals." This number is likely overstated because some people may vary their email address and name, but is much closer to the actual number of unique posters. 
understand how those activities and the social interactions around them were organized through social norms, governance, social roles, and shared perceptions and communicative genres.

I began by reading through all 544 wiki pages and several thousand messages from the following time periods: when the list began, when the wiki was first rolled out, when the wiki was well established, and recent messages. I over-sampled threads that referred to the wiki or list archives in their content. This helped focus in on how the wiki was used by the community (as compared to the archives) in practice. I also oversampled threads that included the word “ADMIN” in their subject header (a label commonly used by administrators when acting in their administrative capacity). This helped locate contentious issues, providing a window into the social norms and group perceptions important to the community.

I grouped together messages and wiki pages that were related to the research questions outlined above and began to write down preliminary facts and hypotheses related to the interplay between the wiki and email list. For example, I looked for evidence that the email list had influenced the wiki and visa versa. I also looked for examples of different types of work that were occurring in the email list and wiki. Early interviews were conducted after this first pass through the data in order to shed light on the preliminary facts and hypotheses, which were abandoned, refined, or strengthened throughout the rest of the data collection and analysis process. Interviews were transcribed on an ongoing basis and common themes addressed by different interviewees were grouped together in order to analyze them side by side.

Based on my initial analysis and the interviews, I decided to perform four followup content analyses. Two of these were genre analyses intended to identify the key communicative genres occurring in the email list and wiki respectively, including their shared purpose and common elements of form. This provided a nuanced view of the ways that each technology was appropriated by the community and the shared understandings 
that evolved around them. The other two analyses codified the reasons that the wiki and email archives were referenced in the community. This helped compare how the two resources were used in the ongoing discussion and their usefulness in meeting certain community needs. It also helped to understand how the two resources influence and draw from one another. These analyses are described in detail below.

\section{Genre Analysis}

In the tradition of Orlikowski and Yates, I use the concept of genre to gain insights into the typical, recurrent communicative actions of a discourse community (i.e., css-d) (1992; 1994). I use their definition of genre as "socially recognized types of communicative actions- such as memos, meetings, expense forms, training seminars that are habitually enacted by members of a community to realize particular social purposes” (Orlikowski \& Yates, 1994, p. 542). Some genres, such as a proposal, are defined primarily by their shared purpose (i.e., to put forward a plan for evaluation and acceptance or rejection) and may take on different forms. Other genres, such as a memo, are defined primarily by their form (i.e., distinctive header, informal tone) and may be enacted for different specific purposes.

It is sometimes useful to consider collections of genres. The entire collection of genres used by a particular discourse community has been referred to as a genre repertoire (Orlikowski \& Yates, 1994). The genres that make up the repertoire and those that are missing shed light on the activities most central to the community (Orlikowski \& Yates, 1994). On a more detailed level, it often makes sense to consider collections of sequentially enacted genres, or genre systems, such as the job advertisement, job letter and resume, and rejection letter (Yates, Orlikowski, \& Rennecker, 1997). In such cases "the system as a whole, as well as the individual genres constituting the system, can be said to have a socially recognized purpose and common characteristics of form” (Yates et al., 1997). 
It is important to stress that "the communicative purpose of a genre is not rooted in a single individual's motive for communicating, but in a purpose that is constructed, recognized, and reinforced within a community” (Miller, as cited in Orlikowski \& Yates, 1994). This focus does not suggest that each individual enacts a particular genre for the same reason (e.g., a member may enact the Announcement genre to demonstrate what an avant-garde CSS developer he is, while another may simply want to inform his friends). Indeed, other studies have explored the range of individual motivations of participating in online technical support communities (e.g., Lakhani \& von Hippel, 2003). While these are informative, they downplay the importance of community norms and shared understanding. In contrast, genre analysis situates an individual communicative act within a social (and technological) context. This draw attention to the ways in which a shared understanding (e.g., genres) can be used as an inhibitor of efficient communication and coordination among community members.

Genre analysis is the process by which genres (and genre systems) are identified and analyzed. In the current study, genre analysis is used to identify existing communicative genres in the community (i.e., characterize the genre repertoire), their characteristics, their purposes, and their relationship to one another and the technology that supports them. Because genre analysis has been used to serve many disciplines and different needs (e.g., help educate writings; help improve information retrieval), the specific techniques used to perform genre analyses differ significantly. For example, researchers have used historical approaches (e.g., Bazerman, 1988; Yates \& Orlikowski, 1992), formal content analysis (e.g., Orlikowski \& Yates, 1994), factor analysis (e.g., Emigh \& Herring, 2005), and various ethnographic methods such as interviews and shadowing (e.g., Dudley-Evans \& St John, 1998; Swales, 1998). Thus, the specific goals of the research project should help determine the specific techniques used to identify, characterize, and analyze the genres. 
The primary goals of the genre analysis of css-d were threefold. The first goal was to help identify and characterize the common activities of the community both in the email list and wiki and how they relate to one another. This helps uncover the shared purposes of communication that are most important to the community as a whole. It also provides some idea of how they are accomplished in practice (i.e., the forms the genres take). It gets directly at the main research question: How is activity organized so as to leverage the email list and wiki repository?

The second goal of the genre analysis is to explore the relationship between the primary activities and the technologies (i.e., media) that are used to support them. Several authors suggest that genre analyses are particularly well suited for exploring this relationship (Chandler, 1997; Erickson, 2000; Yates \& Orlikowski, 1992). Rather than assuming that media are used identically for all activities, genre analysis recognizes that specific media and technological features are appropriated differently to meet different purposes. Several genre analysis studies have demonstrated the value of this approach on informing the design of technologies and social practices (e.g., Erickson, 2000; Yates \& Orlikowski, 2002; Yates et al., 1997), one of the key goals of the project.

Finally, a third goal of the genre analysis was to identify genres that may be of use to other communities (e.g., medical support communities). Prior studies have not yet examined the range of genres that can emerge from a collaboratively authored repository. Although one study identified a collaborative authoring genre system and a collaborative repository genre system as salient genres within workgroups, the technology used to facilitate those genre systems did not allow for the two to be conducted simultaneously as wiki software does (Yates \& Orlikowski, 2002). Because a wiki can be used to support an infinite number of different genres, it was important to identify a subset of genres that had been successfully employed in a wiki repository. These could then be presented as potentially helpful genres to other communities interested in implementing a wiki repository. 
Like other genre analyses, email list and wiki genres were identified by their prevalence and similarity in purpose and form. Identifying genres can be problematic because "a genre is ultimately an abstract conception rather than something that exists empirically in the world” (Feure, 1992, p. 144). Orlikowski \& Yates point out that some genres are identified primarily by their form (e.g., office memo), while others are identified primarily by their purpose (e.g., project proposal) (1994). It is also the case that some genres are more established than others and that they can change over time (Orlikowski \& Yates, 1994). In the end, the best test of the appropriateness of a genre definition is its salience within the specified discourse community and its usefulness as an analytic tool.

I used a combination of approaches to assure that the genres I identified were recognized by the community and useful as analytic tools. First, in the spirit of grounded theory, I let the data suggest the initial genres rather than import definitions from other studies. In this case, data on genres came from my reading of email messages and wiki content, as well as interviews where I asked members to describe the activities and resources. All of the email list genres (discussed in Chapter 4) were identified by the group and verified through my reading of messages. When possible, I used their nomenclature for the genres as suggested (Swales, 1990). Most of them were common to other online communities, although one ("Site Checks”) had not been identified in prior literature.

The wiki genres were not as well established, likely because of the novelty of the wiki repository. This meant that community members did not have a standard terminology for all of the genres (e.g., annotated links), although they did for some (e.g., biography pages). In such cases, there were several clues that helped identify the genres. First, groups of pages were used for a similar purpose in the email list discussion (e.g., to help shut down a fruitless, recurring debate in the conversation). The analysis of messages that referenced the wiki (see below) helped to identify these purposes. Second, 
the organization of wiki pages (e.g., as on the FrontPage) was helpful in determining salient categories from the community’s perspective. For example, they have a section called "Debatable" that includes several pages that I have included in the "Debate Genre.” This was helpful to an extent, but at times pages were grouped by CSS content areas (e.g., Hacks; Tips and Tricks) that included pages of many different genres (i.e., pages that served different purposes and took on different forms). In these cases I strove to identify genres at a level of specificity that would be useful to other communities and were not too specific to the nuances of CSS technology. For example, rather than having a "CSS Layouts” genre, I identified the “Annotated Links” genre, which several of the CSS Layouts pages (and a few other pages like the CssEditors page) fit into. Third, interviews and content analysis of messages and wiki pages helped provide insights into the shared purposes and important elements of form. For example, interviewees described how they used some pages (e.g., ThreeColumnLayouts) to find other websites as if they were a collection of bookmarks. Finally, with the help of a research assistant, I tabulated certain elements of form for each page in order to look for commonalities. These included page size, use of headers, tables, bold, italics, personal pronouns, bullets, and links per page (both external and internal).

After preliminary genres were identified I discussed them with community members in interviews in order to assure that I had accurately represented them and properly understood their purpose as suggested by genre analysis methodologists (Askehave \& Swales, 2001). I also compared them to prior genre studies of email lists and repositories (Emigh \& Herring, 2005; Orlikowski \& Yates, 1994; Yates \& Orlikowski, 2002; Yates et al., 1997) and discussed them with other researchers. This helped to explore alternative genre formulations and issues to consider. This also helped me explore missing genres that one would expect to find in an online support group and wiki repository, an important part of a genre analysis (Orlikowski \& Yates, 1994). 
Once identified, I used prototypical examples of the genres to describe their shared purpose and their common elements of form. The relatively small number of wiki pages allowed me to reread pages several times in order to identify and classify them. In contrast, the email list provided more messages than could possibly be read. In order to identify examples of email list genres I used search strings (e.g., "site check" "holy war" “ADMIN”) to locate messages within various genres. My reading of large blocks of messages provided additional examples that did not have the specific search terms. I also drew upon the interview data and conversations about the genres in the email list discussion to explain how members perceive the genres and how the medium (email list or wiki) affected them.

\section{Coding of Messages that Reference the Wiki \& Archives}

In order to gain a better understanding of how the wiki was used by the community in the email list conversation, I performed a formal content analysis of all messages that referenced the wiki between January 2003 and April 2005. For comparative purposes I also performed a similar analysis of all messages that referenced the email list archive. These messages were identified by regular expression searches for relevant terms (e.g., “wiki," “archive,”) and URLs (including the wiki, private, and public archive URLs). This resulted in 1,787 potential wiki messages and 586 potential archive messages.

I used a grounded theory approach to determine the key reasons why the wiki (or archive) was referenced (Glaser \& Strauss, 1967). I started by reading a subset of these messages and creating preliminary categories that were important in explaining the wiki (or archive) reference. I collected prototypical examples and compared them to aid in the analysis. I continued to modify the original categories through an iterative process until no new categories were needed. At this point, I developed a code book (see Appendix 2) describing the various categories. Categories were not mutually exclusive, as sometimes 
a single message referred to the wiki several times for different reasons. Next, two raters (myself and another graduate student) coded a small sample of messages. After this initial round of coding, we discussed and refined the categories and code book.

Once the categories were established for the final coding, we coded the messages independently using a custom web interface. The interface showed the message that was being coded alongside the list of category names and corresponding buttons. Messages were presented randomly to the coders so that any potential learning bias would be randomly distributed across time. Both raters coded a portion of messages (500 wiki messages and 200 archive messages). This assured that the coding scheme was sufficiently fleshed out and consistently applied. Cohen’s kappa values (k) are reported throughout the paper as a measure of the inter-rater reliability. Scores above .80 are considered high (i.e., they suggest the coders rated messages consistently). When coders disagreed, all positive codes were applied because a review of the disagreements suggested that it was more common to miss a positive code than to use one when it was not intended.

Messages that referred to a different wiki (e.g., Wikipedia) or archive were excluded, as were messages that only referred to the wiki or archive in a quote from a former message (which was not referred to in the current message). This reduced the original 1,787 wiki referencing messages to 1,511 and the original 586 archive referencing messages to 302 messages. Cohen’s kappa scores for these excluded categories were .92 and .94 for the wiki and archive referencing emails respectively. A summary of the final analysis is presented in Table 6.1.

\section{Server Log Analyses}

I performed an analysis of the wiki server logs to determine which wiki pages were most frequently accessed via search engines (see Table 5.2). The most frequently 100 search phrases used during May 2004 and January 2007 that led to the wiki were 
analyzed. Each search phrase was entered into Google (on July 21, 2007) to see which wiki page was returned first. Using Google was appropriate as over $91 \%$ of the searches occurred on Google. I aggregated the numbers in order to show the unique number of wiki pages arrived at from the search engine and the number of searches associated with each page.

I also performed an analysis of the email archive pages visited in July, 2006. The analysis compared the total number of unique archive pages visited with the number of messages in the database during that time period. In addition, the page IDs (which indicate the rank of a message) from the most frequently accessed 1,000 messages were analyzed to determine if older or newer messages were more frequently visited.

\section{Additional Analyses}

Throughout this process, I conducted various other ad hoc analyses to help validate (or disconfirm) hypotheses generated from various sources. For example, I analyzed all of the Site Check pages on the wiki to see how many times they had been edited and how many comments they received from different authors in order to show that they have not been successful. An analysis of the RecentChanges wiki page suggested that most edits were small in nature (e.g., "fixed typo", “added link”), a fact that was corroborated by the statistics on page growth over time and interviews. I also captured the history of certain wiki pages (e.g., IE7 page) in real time to get a better understanding of the short-term activity on the wiki. Another analysis was performed to

measure the correlations between wiki page edits, their size, and the number of times they were referenced. Throughout this entire process preliminary findings were vetted to other researchers familiar with the study, written up, and presented to css-d community members in order to strengthen the arguments and consider alternate explanations.

\section{Ethical Considerations}


Before analyzing any data I received IRB approval to work with css-d and approval from the css-d administrator and community as a whole. Community member names and personally identifiable information are not presented in this thesis. When possible, I received approval to use direct quotes from the author of the email list messages. When not possible the contents of the messages were combined or modified slightly to make the author less identifiable.

\section{Conclusions}

This chapter has introduced an empirical study of a technical support community (css-d) and described the goals, research questions, data sources, and methodology of the study. The next 4 chapters present findings from the study. The primary research question examined in the study was: How is activity organized to leverage both the threaded conversation and the wiki repository? The research question was addressed by using a mixed method approach relying upon a number of different data sources and iteratively examining and comparing them. These included content analysis (some of which was formalized through coding of messages), quantitative analysis of participation patterns, an analysis of wiki server log reports, semi-structured interviews, and a genre analysis. This mixture of methods allowed me to look at the primary activities occurring at css- $d$ (within both the email list and wiki), the types of work necessary to perform those activities, and the ways in which the email list and wiki draw from one another. It also provided a glimpse of the ways that people use the wiki within the ongoing conversation and as a secondary resource. 


\section{CHAPTER 4}

\section{CSS-D EMAIL LIST}

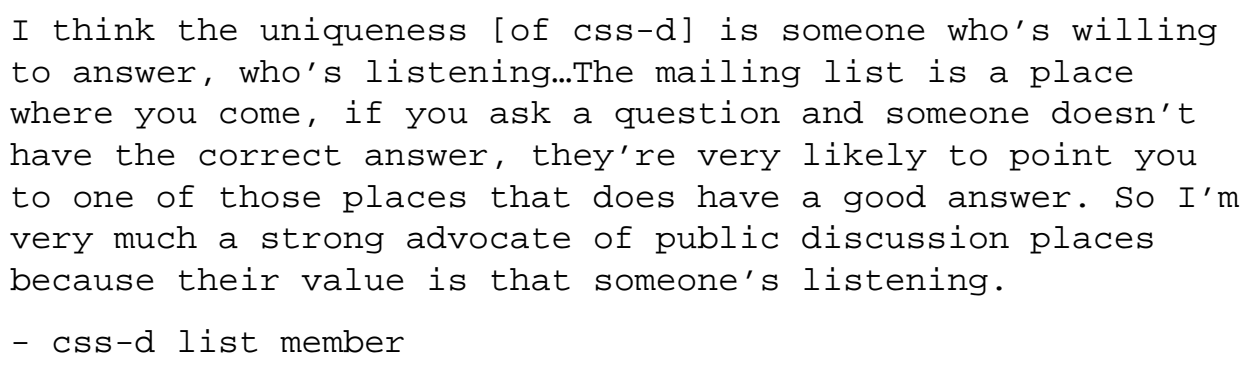

The email list is the backbone of the css-d community. It is the place where the majority of the action takes place. The purpose of this chapter is to provide a nuanced look at the primary activities and social roles found within the email list. Throughout the chapter, I discuss the implications of the current arrangement on the reuse of information that is shared within the community. The chapter includes the following sections:

- History

- Technical Infrastructure

- Governance

- Genres and Activities

- Participation and Community Roles

- $\quad$ Reuse of the Discussion Archives

- Conclusions

\section{History}

The css-d email list was started by a well-known web design expert in January 2002. In contrast to more general purpose web design email lists (e.g., webL), css-d was 
limited to discussing CSS technology and specifically, the practical use of CSS in web design. There were over 650 members within just 2 days and over 1,250 within the first month (see Appendix 1 for later subscription numbers that were stated by the administrators periodically in messages to the list). Many initial members mentioned finding out about the new list from other web design email lists such as webL, as well as from the personal websites of the founder and other web design gurus. Interviewees suggested that the large number of subscribers was attributed to the notoriety of the founder, as well as pent up demand for a CSS-only email list.

The founder expressed his excitement and surprise at the initial success of the list in a message posted a few weeks after its creation: "With 1,252 subscribers as I send this, it's obvious that interest in CSS is quite high indeed, much higher than I ever would have dared hope.” This comment hints at the somewhat marginalized position that CSS played at that time in the world of web design. Although the original CSS1 specification had been around since 1996 (Lie \& Bos, 1996), early browsers only half-heartedly supported it. Internet Explorer 6, which supported CSS much better than prior versions, had been around since October 2001, so using CSS had recently become more practical. However, debates about the value of using CSS for layout purposes (as opposed to HTML tables) were common at this time. Although a popular website design site implemented its website using CSS in February 2001, it wasn’t until October of 2002 that the first major corporate website, Wired.com, fully adopted CSS for its site’s design. The fact that Wired considered its accomplishment worth a press release suggests that CSS was still largely marginalized at the time (Rochmis) (see Appendix 1 for CSS and css-d timeline).

Although CSS is now central to website design and used on countless personal and professional sites, its initial marginalization likely contributed to the sense of community among initial css-d members. Each CSS-related problem that they solved became an argument in favor of using CSS, and each newcomer they helped became a potential CSS advocate. The less marginalized CSS became, the more likely it would be 
supported by future browser releases, design tools, and web technologies. Furthermore, the skills of those that knew CSS would be in greater demand.

The email list has continued to grow in membership to over 7,500 members as of July 2006. This growth prompted the original administrators to solicit the help of additional volunteer moderators in November of 2004. The growth also led to several significant changes to the email list technical infrastructure. First, the email list's growth required migration to new servers hosted for free by Incutio, a web design company, beginning in December of 2002 (almost a year after its creation). Second, the high volume of messages prompted a few members to suggest that an email archive, wiki, or FAQ page be created. As a result, one of the active list members created a searchable, public archive of list messages and a community wiki in August of 2002, both of which are still in use. Even with these changes, interviewees suggest that the list's focus has not changed over time, although some suggest that it has become more focused on helping newcomers and less tolerant of off-topic discussion over time.

\section{Technical Infrastructure}

The css-d email list has always used the Mailman email list management tool (Viega, Warsaw, \& Manheimer, 1998). Anyone can subscribe to the list (i.e., become a member) by providing a valid email address and password. There is no manual gatekeeping to assure that those who register fit certain criteria. Once subscribed, a member can send messages to the group email address, which will be sent to all other subscribed users. In order to reduce spam, non-subscribed users may not post to the list. Messages sent to the list include “[css-d]” in the subject line for easy identification.

Each individual is free to use any email client of her choosing to read the messages. Discussions about managing email using various email clients crop up occasionally, especially when list policies such as quoting too much material are violated. 
Although there is variety in how people view messages, css-d is a typical example of threaded conversation as described in Chapter 2.

\section{Digest Mode}

Members can receive messages in a daily “digest” rather than receiving each message individually. With around 50 messages a day the digest can be quite large, which is one reason members are encouraged to reduce the amount of quoted material in their messages. Digest users often ask those replying to their messages to copy them in directly so they don't have to wait until the digest comes. Digest subscribers who reply to messages are encouraged to change the subject line to a meaningful one rather than the default digest subject line (i.e., “[css-d] css-d Digest, Vol 39, Issue 46”). However, this is not always practiced.

\section{List Configuration}

The list is unmoderated, meaning that messages are not reviewed by any members before being sent to the list. This means that troublesome messages can only be dealt with after-the-fact.

The default "Reply-To" behavior is to reply to the sender, NOT to the email list. An individual must choose "Reply-to-All” (in his email client) in order to send his reply to the entire list. This decision is not universally appreciated, but is strongly defended by the list administrators. The hope is that people will only send messages to the list when it is of general interest. Interviews revealed that many messages are, in fact, sent to individuals directly.

No attachments are allowed on the email list. When there is a need to share a file (e.g., a CSS file), members typically post it to the web and include a URL in the message. Because the list is for website developers they can typically do this without much problem. 


\section{Private Archives}

All messages sent to the list are included in the private email archive. The archive is accessible to subscribers through the community website. It includes the contents of each message sent to the list. Members can browse messages in the archive by month. For each month, messages can be sorted by thread, subject, author, or date. In addition, members can download text files including all messages for a given month. These files are not easily imported into email client software. The result is that there is no easy way to search the entire private archive. One must search each month separately by using the search functionality built into browsers or text editors or simply browse through each month individually.

\section{Public Archives}

Because of the limitations of the private archive, a list member created a public archive that is searchable in August of 2002. Messages prior to that time were also made available. The archive removes all email addresses included in the header or body of each message so that email spam bots could not harvest them. Recent messages are shown in reverse chronological order with the subject line (which links to the content of the message), author, and date displayed. Visitors can browse messages by month or search the entire database via the search box at the top of the page.

The public archives were an important addition to the mailing list for two primary reasons. First, it made the contents of the email list accessible to a much wider audience, especially since search engines could index the contents. The result was that many people were introduced to the list through search engines. Second, the entire email archive became searchable. This allowed newcomers to more efficiently learn from the prior experiences of the list members. Unfortunately, the search functionality does not work as many individuals expect, as indicated by the many complaints about it. Specifically, if 
more than one search term is entered the Boolean OR operator is used rather than the AND operator. This results in searches with low precision. To overcome this problem members recommend using Google’s site search feature to search the public archive.

\section{Governance}

Like many online support communities, css-d relies upon volunteer administrators, policies, and social norms to help maintain social order. The distributed, voluntary, computer-mediated environment of email lists pose many new social challenges and old challenges in new guises. For example, the combination of anonymity and lack of social cues due to text-only conversation can lead to an increase in hostile messages, or flames (Dery, 1993). Other social challenges common to css-d, as well as other online communities, include motivating participation, keeping the discussion ontopic (Kollock \& Smith, 1996), and preventing offensive behaviors such as over-quoting of prior messages, asking frequently asked questions, and misusing subject headers (McLaughlin, Osborne, \& Smith, 1995).

Several experienced online community administrators and authors have recognized the need for strategies and technologies that help address these social challenges within online support communities. For example, Preece (2000) argues that the need to design for "sociability" is at least as important as the need to design for "usability.” Other popular books on building online communities focus on fostering appropriate social interactions, rather than the details of the technologies (Kim, 1999;

Powazek, 2002). Likewise, the Listserv ${ }^{\circledR}$ manual provides detailed suggestions for email list owners (i.e., administrators) on how to deal with flame wars, promote proper “netiquette,” and welcome newcomers ("LISTSERV Introductory List Owner's Guide," 2003). Butler, et al. (2002) use a survey methodology to characterize the current work and motivations of several email list administrators and members. They found that list administrators play a unique role within communities - one that encompasses both 
technical and social responsibilities. This is true at css-d as well as described in the following section. They also found that other members shared in the community building activities through participation, recruitment of new members, and even managing social dynamics. This is also true at css-d, where members play an active role in helping maintaining social order through their use of snide remarks, gentle reminders, and on occasion the use of the silent treatment (i.e., ignoring a message that blatantly violates community norms). In Chapters 5 and 6, I will discuss the additional activities of administrators and core members as they relate to a wiki repository.

\section{Administrators}

The css-d list is notably influenced by the active role of the list "chaperone" and a handful of “admins” who keep the discussion running smoothly. The admins are all longtime, active community members who are generally well respected CSS experts, as evidenced by the many times members positively refer to their work in the list. The original list founder was largely responsible for establishing the values, tone, and scope of the list. He considered himself a "benevolent dictator" and took on the name "list chaperone” after a community-wide naming contest because it captured his own perception of his role.

The css-d email list's policies and scope were established during the first month by the list founder, with some input from other list members. They have not changed significantly since, although their enforcement was increased after the list founder solicited the help of 4 volunteer administrators in November 2004. The policies were originally posted to the list (in a series of email messages by the founder) and added to the founder's personal website. Later, they were added to a css-d website ("Cssdiscuss.org," 2002) and the wiki. Some of them were imported from other lists, while others were developed as a result of problems that occurred at css-d. Although this is not always the case, at css-d the formal written policies and values are fairly consistent with 
the actual experience and perceptions of list members. The following sections describe these policies and guiding principles in detail.

In addition to managing email list software (e.g., keeping the membership list current), admins deal with the typical social maintenance issues that come up on unmoderated email discussions. Admins post public messages at least weekly to remind members of list policies (e.g., trimming quoted material) and to keep the discussion friendly and on-topic. These messages often include the word "ADMINS:” in the subject line and are typically sent in reply to the offending message(s). They generally include an appeal to the list rules and policies, state the authority of the administrator, and sometimes offer arguments about the rationale for the policy. Admins also send messages in private, especially for more minor or private offenses (e.g., asking a new question in an existing thread). Although the threat of removing individuals from the email list is mentioned regularly, it is only followed through with on rare occasions for the most egregious of sins (e.g., posting job solicitations or automatic replies). This act is tempered by the fact that members can re-subscribe and in many cases do (presumably having learned their lesson). Currently, administrators take turns monitoring the email list on given days so that they can be fairly responsive to problems that may arise.

Challenges to the admins' authority are rare and are encouraged to occur offline. Several members have complained to me personally or in public messages posted to the list that the admins are too authoritarian. However, others have defended the admins’ approach in public messages and interviews.

\section{List Policies}

The list policies define acceptable and unacceptable activities. Their purpose is to improve the signal-to-noise ratio, keep the discussion friendly and focused, and explain the requirements of the software. The policies are included in their entirety at the main css-d website ("Css-discuss.org," 2002). I have summarized them below: 
- trim quoted material in emails and keep signature files short

- avoid auto-reply messages, job solicitations, advertisements, unsubscribe requests, "test” messages, and cross-posting

- don't send attachments or HTML email to the list

- avoid "flaming", being offensive, and taking offense

- post answers below a quote of the original question, rather than posting the answer above the question ("Jeopardy Style") [this is a strong suggestion, not an official policy]

- don't republish messages without permission from the message author or list administrator (including on automatic gateways such as SMTP to NNTP gateways)

In addition, the policies clarify the following points:

- messages cannot be deleted from the archives

- individuals should send messages from the email address that they registered with

- if your email address "bounces" you will be removed from the list

The policies conclude by stating that "People who violate the goodwill of the list community will be unsubscribed with extreme prejudice. Not to mention haste.”

In addition to these policies, the css-d website includes information about the scope and purpose of the email list more generally. The following three sections describe the recommendations and the actual practices related to these important governance issues.

\section{Establishing the Boundaries of the List (i.e., determining what is On and Off-Topic)}

One of the most significant challenges many help-based communities face is the need to keep the discussion on-topic. Members who come to learn about the community topic (e.g., CSS) can easily become frustrated when the community conversation drifts too far from its stated focus because they must filter out off-topic messages. Kollock \& Smith (1996) argue that staying on-topic is critical to the coordination of community 
knowledge sharing, but unfortunately, is challenged by the free-rider problem. In other words, while the collective good is best served by people staying on-topic, there is an individual temptation to post off-topic messages in order to reach more people. Other more innocent posters may simply not understand what constitutes an on or off-topic post. For example, at css-d many well-intentioned individuals post off-topic messages because it is not obvious to them what distinguishes a practical CSS question (which is on-topic) from a theoretical one (which is not). In addition, there are many gray areas at the intersection of CSS and related technologies (e.g., using a scripting language to serve different CSS pages to different browsers). Finally, some people post off-topic messages because they have formed friendships with community members and want to share meaningful experiences with them, even if they aren't directly related to the topic at hand.

At css-d the first month was particularly important in establishing what is on and off-topic. When asked if the boundaries will "evolve naturally" or if there were "predefined markers," the list founder indicated that he was "counting on a natural evolution, as happens with all lists.” However, after only a week some members vented their frustration at the amount of theoretical discussion and pointless debate to the list founder. The list founder recognized this as the "first constitutional crisis" and agreed that "the primary purpose of the list, to give practical advice and help to newer authors becoming more skilled with CSS, is in danger of being lost.” He provided some examples of message he considered off-topic (e.g., debates about the role of CSS and "standards", client relations, and which tool is best) and suggested places where they are more appropriately addressed. Later messages were sent out to reinforce the original purpose of the list and the list policies were updated to read as follows:

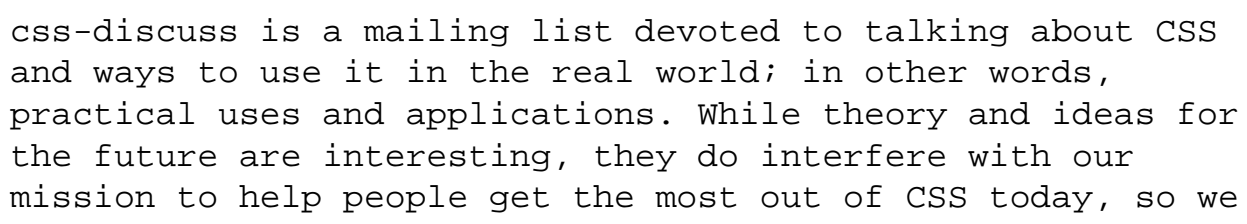


tend not to spend a lot of time on theory. There are other

lists and groups for that sort of thing anyway.

Over time, the list founder, other core members, and later list administrators have regularly posted messages warning members when they have drifted too far off-topic. Their vigilance has not been universally appreciated. One member volunteered this information to me after I announced my project to the list: "Personally I think the list moderators are a bit anal about what people talk about on this list. Anything that seems a hair off-topic is shutdown... Sometimes you can actually be talking about css and still be kicked off.” List administrators also mentioned this as a sore spot for many members, but also felt there was support for their position, which was confirmed by other interviewees. They appreciate the fact that css-d, as it is currently defined, fills a unique niche in the broader CSS landscape and don’t want to see it lose its focus.

Members who have something to say that may not be strictly on-topic have adopted several strategies for sharing their comments in acceptable ways. One common strategy is to bundle their off-topic comments with a useful one. When the off-topic content is not too far astray, this strategy is tolerated (see next section for a related discussion). Another strategy is to send off-topic messages directly to the other list members. For example, when someone asks how to serve different CSS stylesheets to users with different browsers, a replier can send javascript code directly to the requester. The downside of this is that the information sent to them is not peer reviewed by the entire list. Finally, some members have used the community wiki to post off-topic, but related content as described in detail in Chapter 5.

\section{Supporting Newcomers}

One of the core values of css-d has been the need to support newcomers. In fact, the list founder mentioned in his interview that this was the primary reason for the list to be created. Although there were some Usenet groups that discussed CSS, they were 
becoming "beginner hostile" and there was a tendency to reply to basic questions with "an answer that pretty much ran verbatim: go read the specifications, URL and that was it.” The list policies express css-d's different approach best:

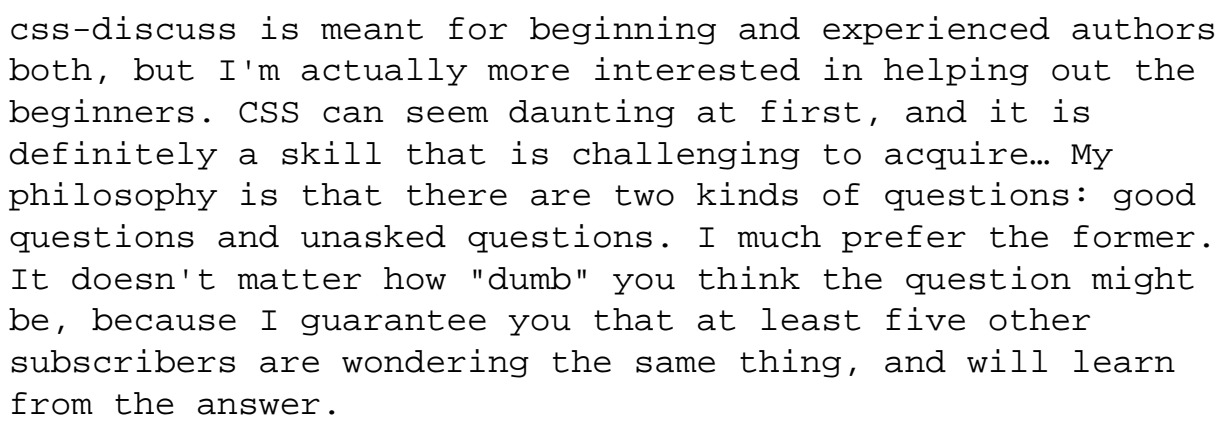

The list policies go on to suggest how members should answer novice questions:

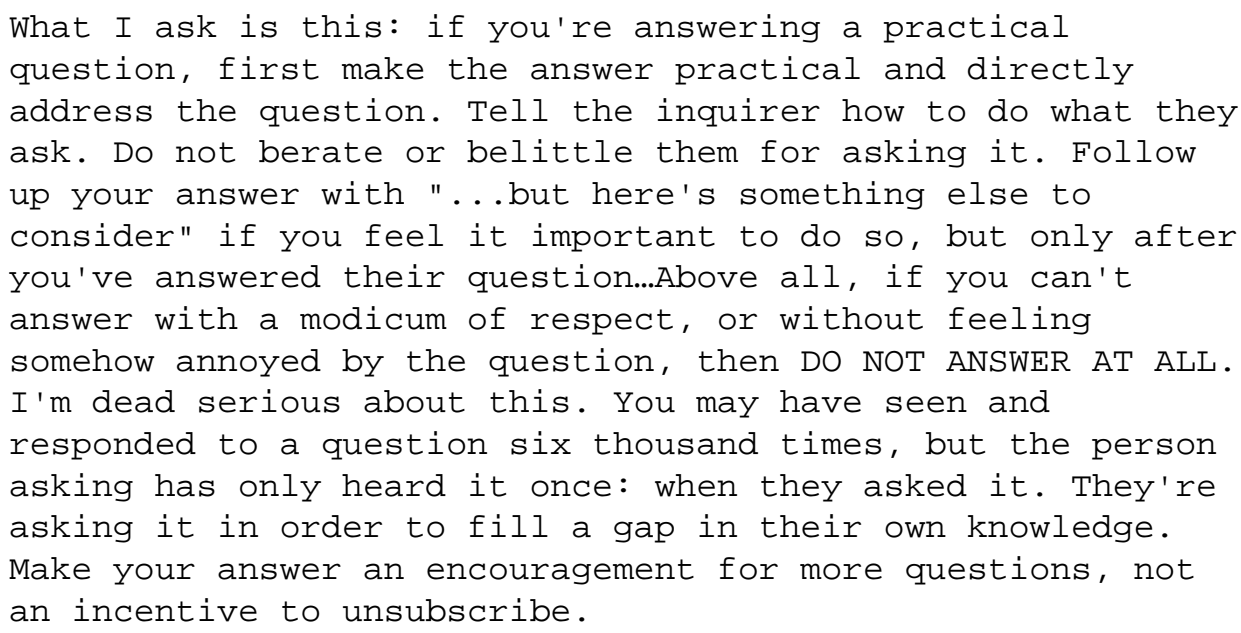

For the most part, the list has followed this counsel. Nearly all interviewees mentioned the friendly and welcoming attitude towards novices as a key difference between css-d and other email lists that they have been a part of. On the rare occasions when a member does belittle a novice for asking a frequently asked question, other members quickly jump in and chastise the belittler. The welcoming attitude towards newcomers may at least partly explain the continued increase in membership, as newcomers are more likely to stick around if they had a good experience (Lampe \& Johnston 2005). Some interviewees who are now core members mentioned the warm welcome they received as a major motivation for their continued participation. However, 
interviews revealed that a few core members have left because they felt like the level of discussion had degraded (at least in part due to the many basic questions getting asked by novices).

\section{Dealing with Frequently Asked Questions}

Because of the constant influx of new members in many online communities, the same questions are bound to come up repeatedly. Communities vary in their tolerance for repeat questions and their approaches to overcoming the challenge. Although css-d's leadership wants to support newcomers and their basic questions, they also recognized that the list needed to keep the discussion interesting enough for the more experienced members and not too burdensome. The official policy reads:

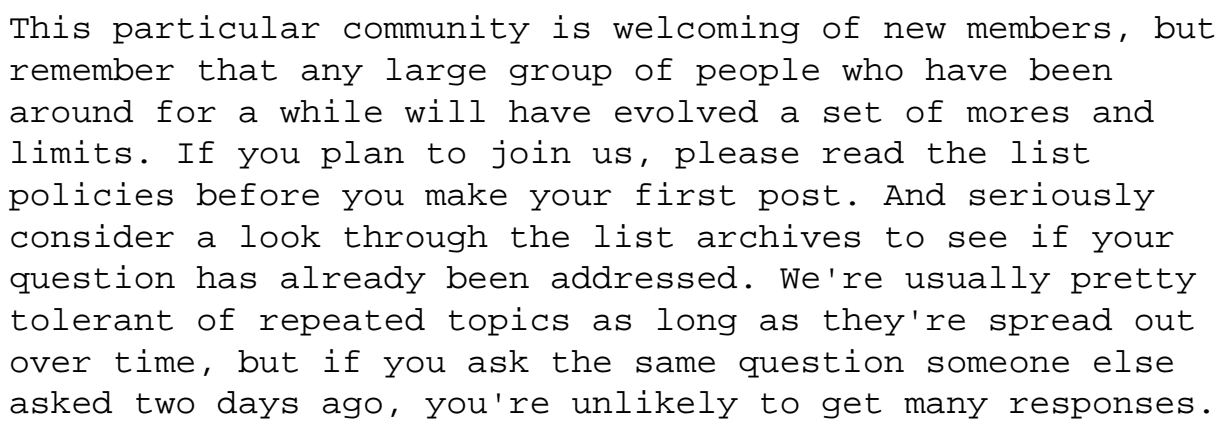

This statement was written when only the private archives were available; a time when performing a comprehensive search on a topic was not very practical. Early on, members asked for copies of old digests, ways of downloading the private archives, and if an FAQ document for the list existed to help overcome the limitations of the private archive. Some members suggested creating an FAQ document or wiki when they learned that one didn't exist as in the following message sent only a month after the list was created:

This list is too prolific and informative; I'm drowning under the volume of messages. Judging from a number of repeat questions floating by I'm not the only one.

The archives at [URL for private archives] are a great help but they aren't searchable. 
Is there anybody with bandwith and some space to spare who would consider putting up a wiki? or at least a FAQ page?

Although some members volunteered to set one up, nothing was acted upon initially. This may have been because members pointed to an existing wiki called AllMyFAQs that had been around for a while and covered website design topics including CSS.

Six months later, list members raised the issue again because of the many repeat questions and it ultimately led to the creation of the public list archives and the css-d wiki (see timeline in Appendix 1). Although members were always encouraged to read the private archive before posting, the public archive and wiki made this more practical and members pointed FAQ askers to those resources fairly often. However, the initial tone of friendliness to newcomers was still enforced, as evidenced by the fact that of the 331 messages that sent people to the public archives only 9 (2.7\%) explicitly stated that they should check the archives before posting $(k=0.78)$. Likewise, only 20 of the 1,308 messages (1.5\%) that referenced the wiki explicitly stated that the wiki should have been consulted first $(\mathrm{k}=1)$ (see Table 6.1). Instead of chiding newcomers for not having looked in these resources, the typical response was to simply answer the question or link to the archive or wiki (often with a comment about its general usefulness) and provide a few sentences about how it applies to the individual.

This general approach of including more than just a URL as an answer was encouraged by the list founder in the policies and early messages sent to the list, which stated:

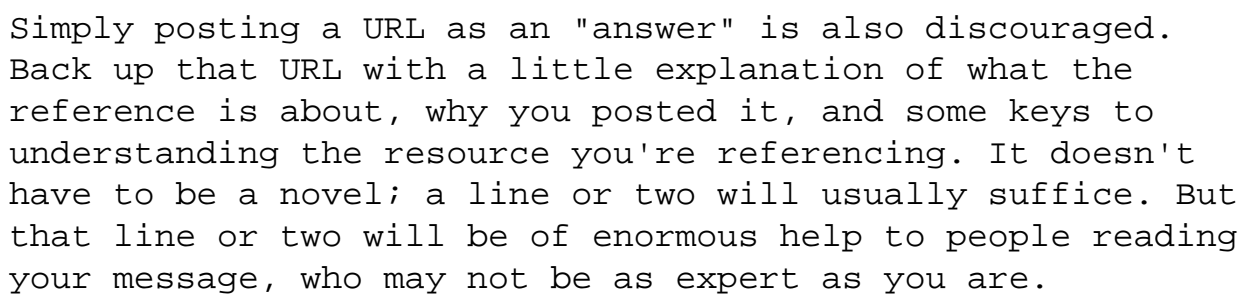


The approach was summarized by a list member in the following post, sent as a reply to the administrator's original message outlining the policy:

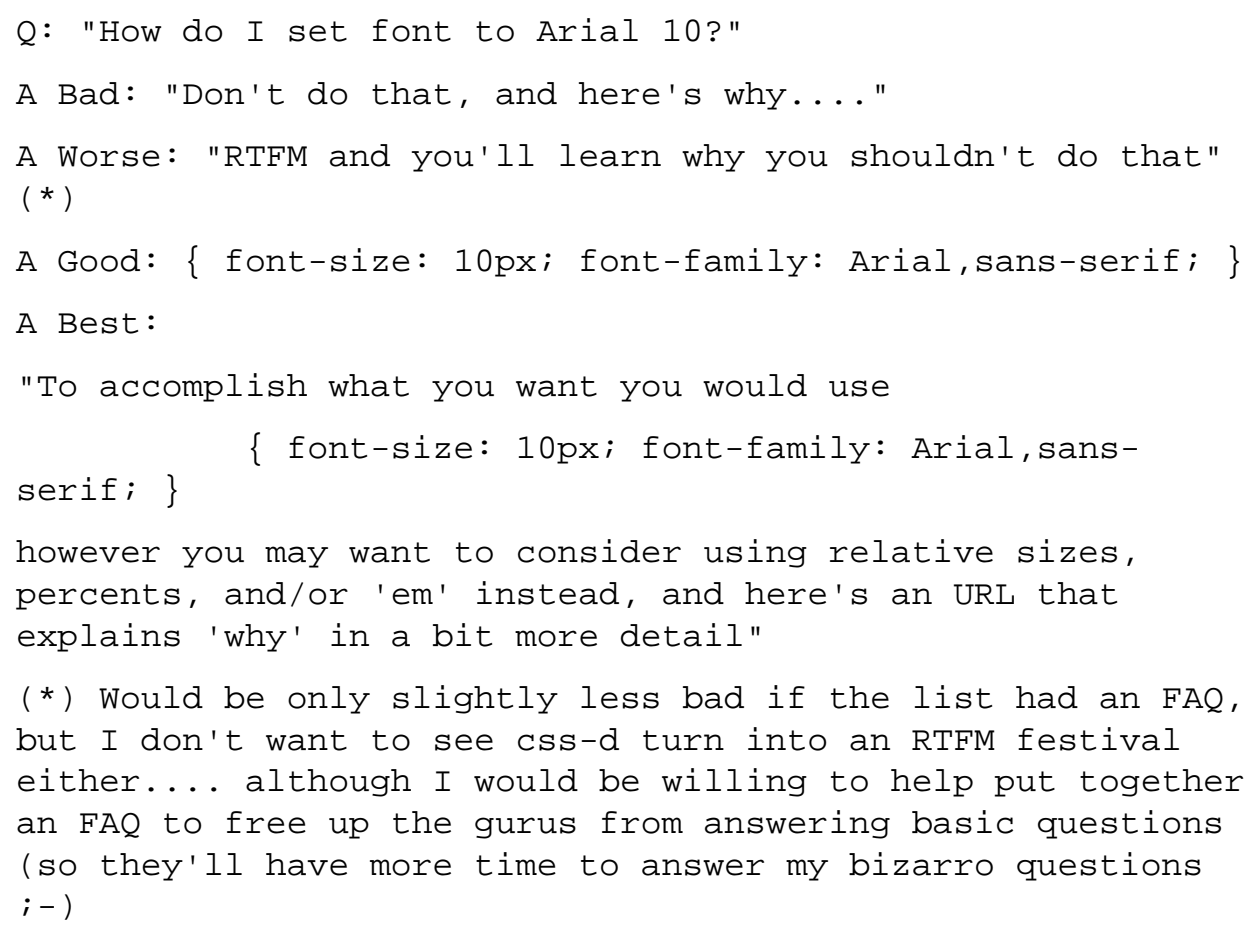

In this example a basic, newcomer question is asked and several different approaches to answering the message are compared. The "worst" approach includes a harsh statement that the questioner should Read The Flipping Manual (polite form of RTFM) without actually addressing the question. The comment indicated by the “*” suggests that in this case a helpful "manual” (i.e., FAQ document) does not exist. This message was written when the searchable public archive was not yet available.

There is evidence that some members (although certainly not all) do consult the archive or wiki before posting. One interviewee mentioned that he rarely posted questions to the list because he found answers to most all of his questions in the wiki and archive. Although hard data is not available to definitively prove that fewer frequently asked questions arise on the list since the wiki and archive came along, two long-time members mentioned that they felt like the quality of questions had increased since those resources became available. Coding revealed 227 cases where the questioner explicitly 
mentioned that they had consulted the wiki before posting and 60 cases where they explicitly mentioned consulting the public archive (see Table 6.1). Although these numbers represent only $2 \%$ of all first posts (most of which are questions), there are likely many cases where people consulted the resources but did not feel the need to explicitly say so in their message. The fact that many members don’t feel obligated to justify their question is likely a reflection of the welcoming attitude the list takes towards newcomers.

\section{Genres and Activities}

As in most threaded conversations, activity is organized around threads. A thread is started when a member posts a message to the list with a new subject header. At css-d this is most often a question, request for feedback on a web site the author created, or an announcement to the list. Members typically continue the thread by replying to the original message, as well as replying to others who have replied. Thus, each thread ideally stands on its own as a brief conversation about the issues brought up in the original post. However, as discussed in the prior section, members posting to the list are encouraged to read related messages in the public archive or related wiki pages. In this sense, each thread fits into a larger information context. Although some members that were interviewed indicated that they read every message, others indicated that they selectively read messages based on the subject header. For this reason, meaningful subject headers are encouraged and some norms around their use have been established (as described below), although they are not explicitly mentioned in list policies or commonly enforced by administrators.

On average, threads are 3.3 messages long, although threads can include dozens of messages - especially when a contentious topic is raised. This is comparable to other active online support communities (Butler, 2001; Galegher et al., 1998; Lakhani \& von 
Hippel, 2003). Although threads often last for days, it is not uncommon for replies to be sent within minutes.

\section{Css-d Genre Repertoire}

As discussed in Chapter 3, the characterization of threads and messages into communicative genres can be a useful way of understanding the shared activities of a community. The use of shared, communicative genres helps coordinate activity and improve the efficiency of communication (Yates \& Orlikowski, 1992). In this section I characterize the key communicative genres found at css-d as a way of describing the major shared activities. Later, I discuss the social roles and individual participation of members.

Our study of existing communicative genres enacted in the css-d email list identified three conceptually unique genre systems: Question and Answer (Q\&A), Site Checks, and Holy Wars. In addition, two genres at the individual message level were identified: Announcements and Administrative. Each of these is described below by focusing on the primary situation in which they are invoked, their substance (e.g., social motives, purposes, and topics being discussed), and their form (e.g., common physical and linguistic features). Although it is meaningful to talk about these as being distinct genres (and genre systems), in practice multiple genres can occur in the same email thread or even email message. Thus, a person answering a question (as part of a Q\&A genre) may begin a Holy War by providing a provocative statement about best practices. Thus, our selection of genres and genre systems is not an attempt to create a mutually exclusive classification scheme for threads or messages. Instead, it is an analytical tool used to describe the communicative actions most salient to the community itself.

\section{Q\&A Genre}


By far, the most common communicative form is the Q\&A genre system. This genre system begins when a list member recognizes an information need and brings it to the community. The typical (and suggested) practice is for her to post her question in a new thread. The new thread signals to other members that there is a new question to be addressed. Later messages reply to the initial thread providing answers, hints, comments, and links to useful references, as well as commentary on other members' suggested solutions. Below is a discussion of the question asking and answering portions of the Q\&A genre.

\section{Question Asking}

The following example is typical of a beginning message in the Q\&A genre:

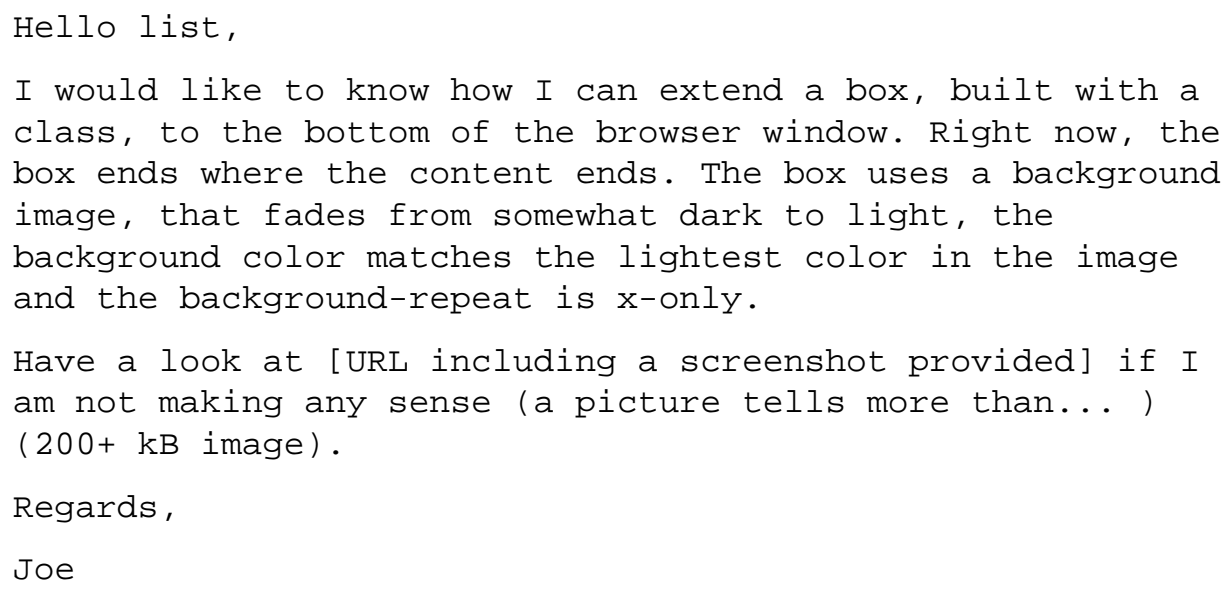

In this example, Joe is unable to display the structure of a website in the desired way. He includes a brief salutation and signs his comment as is typical. The question asks how to accomplish a particular effect (i.e., extend a box when content doesn't fill it up). As is common, he provides some details about the context of his question and a link to a screenshot displaying the actual problem. It is also common to link directly to URLs and CSS files. His comment, "a picture tells more than...” underscores the visual nature of website design and the weaknesses of text-only communication. Other authors also 
struggle to find appropriate words for certain behaviors such as the "weird, bouncy disco dance” that occurs when clicking on an input field.

Providing a link to the website being discussed is a very common practice. The list policies say it this way:

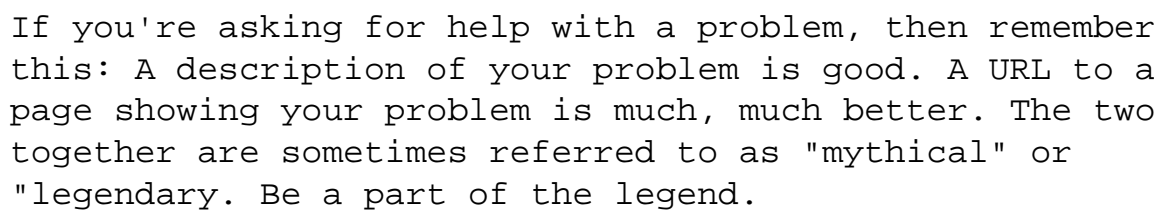

This official policy is constantly reinforced in the ongoing discussion.

Newcomers see others frequently posting URLs to help explain a problem or solution.

When members fail to post a URL when it would have been useful, they are often encouraged to do so as in the following example:

Now I have to apologize, because even with your explanation and the code you provided, you lost me. Is it possible for you to provide a URL to the page in question so we can give it a look see? If the content is restricted, strip it out and replace it with dummy text. Working with the actual page generally offers the best opportunity for someone to provide helpful advice.

While this practice of providing URLs of webpages is central to the work of asking and answering questions, it causes problems related to the reuse of the information. The problem is that most of the URLs are temporary, or get updated. The email list includes many examples of times when a member visited a URL to help answer a question, only to find out that the URL no longer displayed the problem.

Another typical example of a question is provided below:

Hello,

Well I did some google-searching, and some css-d-archivesearching but couldn't find answer to my query. A possible reason for this is because I have no idea how to describe the problem to the search engines and... so that might be why I didn't find anything.

What's happening is that until I apply 'border' to a div that contains an unordered list whose elements are floated left, the div is not the height I would expect. It's as if 


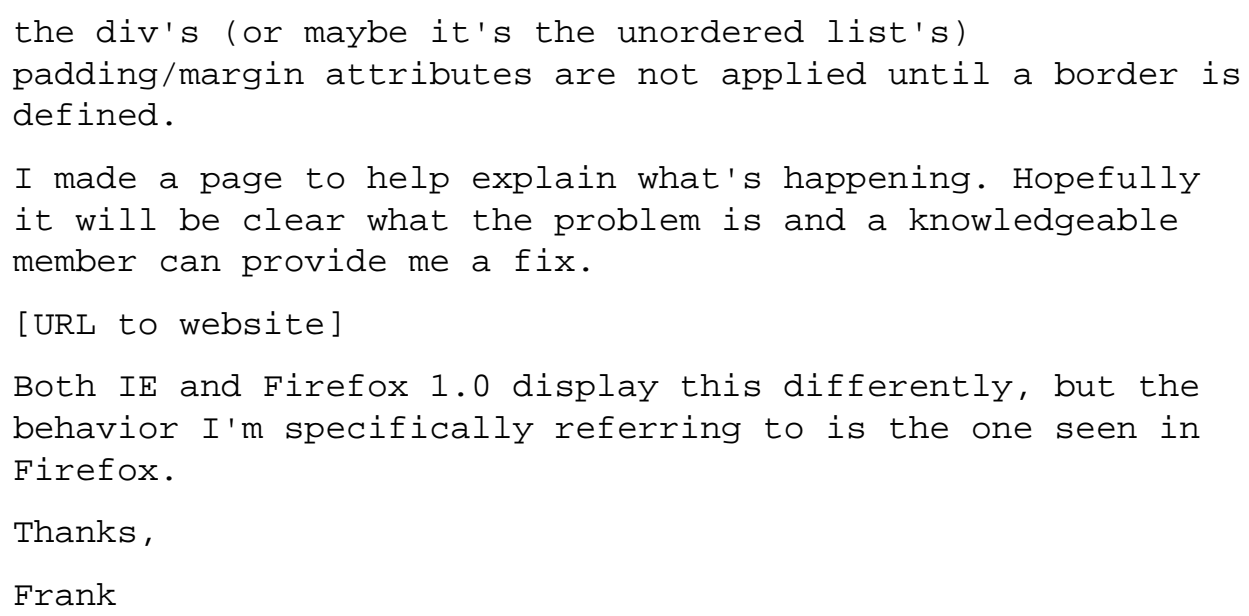

In this example, Frank is trying to diagnose an unexplained behavior related to the spacing of material on a website. This is a common type of problem discussed on the list. Frank mentions the difficulty of searching for this type of problem. This difficulty arises from the visual nature of the problem and the complex situation and numerous factors that could cause the unexpected behavior to occur. Other messages also express this difficulty (e.g., "I attempted to search the archives/Wiki on this but realized I didn't know *what* exactly to search for...). Although diagnosing problems like this one pose a difficult information retrieval problem, CSS experts are often able to quickly recognize the underlying problem because of prior experience and content expertise (see the following section for the resolution to this one).

This example also highlights the common practice of including information that is anticipated to be of use to those answering the question. As in the prior example, he links to a page that demonstrates the effect since it is visual in nature. He mentions that his page was created in order to "help explain what's happening.” This is a suggested practice - to create a "test case" web page that displays the problem, but not any other CSS code. This makes it so other members don’t have to look through pages of CSS code to understand the problem. Frank also includes a tentative hypothesis of the reason behind the unexplained behavior (“It's as if the...”). This may suggest ideas to readers that would help them answer the question. In addition, he provides information about 
which browsers display the problem and suggests which one he is most interested in. This helps members understand his particular situation and needs.

Note that both of the examples are the result of an immediate information need and are presented in a highly contextualized manner. This is the norm. Questions include stories about how the person ran into the problem, what solutions they've tried or initially thought were valid, and even occasionally why it is so important that they find an answer. These details make the interaction more personal and meaningful, as well as provide insights about the underlying information need, not simply the compromised need that is presented to the community after it has been recast in anticipation of what they can offer (Taylor, 1968).

Personal comments are also used to also help justify why a message was posted to the list and why it deserves an answer. Members explain that they have searched for the answer elsewhere but ran into dead ends, or are confused by what they've read. They also provide assurances that replies will be well appreciated (e.g., "any assistance well appreciated”). On occasion they also make comments about their intention to help others (by answering questions) once they know enough themselves.

\section{Question Answering}

Questions are typically answered as reply messages sent either to the individual or to the list as a whole. Some members, including at least two admins, check to see if all of the questions received at least one reply. If they haven't, the members will answer them or "point them to where they'll find more information.” Thus, nearly all questions have at least one reply message. Answering can occur within minutes or sometimes not for days, although most messages are addressed within the first day. The following example is a reply message sent by a CSS expert within 20 minutes of the original question (see Frank’s question above). 


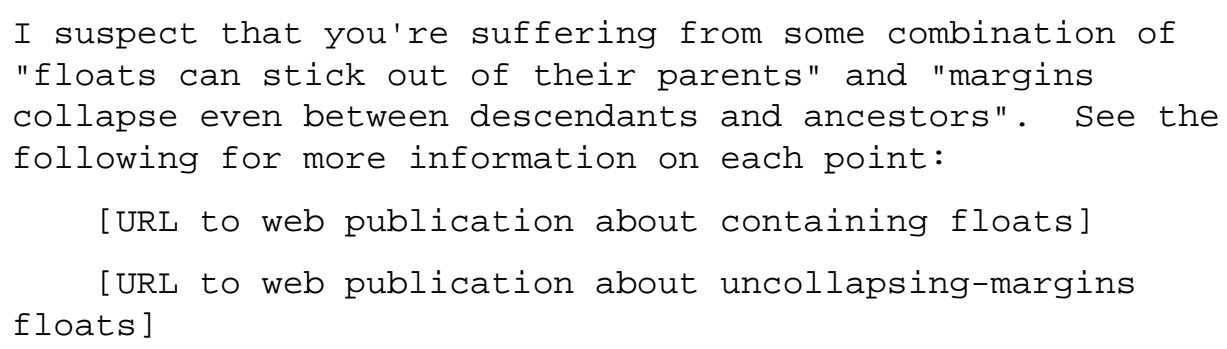

This example highlights the value of human to human interaction that is supported through the email list. Diagnosing a complex problem is a canonical example of the type of work that human experts are adept at (Anderson, 2004). It is also the type of work that is most difficult to capture and codify in written materials and even expert systems (Dreyfus \& Dreyfus, 2005). Here, the question that was so difficult to even explain in words was quickly recognized by an expert.

This example also highlights a common way that resources are used on the email list to help answer questions. Here, the responder's primary contributions were recognizing the underlying cause of the problem and identifying resources that are helpful in overcoming that problem. For an expert familiar with the problem and resources, this activity takes relatively little time (in this case less than 20 minutes). If the resources are indeed helpful in explaining the problem and how to overcome it, then this approach is sufficient to help the questioner without the need to write a treatise on the topic in the email message. If it is not sufficient (as sometimes occurs), the questioner can post a more informed follow up message using terms with which other members are more familiar. The usefulness of simply providing appropriate resources (at least for some individuals) is suggested by the many messages that explicitly ask for a link such as the following ones:

- Can some one please point me to a fix/hack?

- A link to a URL or prior message would be great. 
As discussed in the "Dealing with Frequently Asked Questions” section above, members typically provide a sentence or two providing context for the URLs that are provided as in the reply to Frank's message.

Like questions, answer messages are typically provided in a highly contextualized way couched in the terminology of the current problem at hand (e.g., "You are confusing ID's (\#foo) with classes (.foo)...”). Some members go to extreme lengths to provide personalized answers to questions. It is not uncommon for members to download a questioner's CSS page, modify the code to fix a problem, and then provide the modified code to the original questioner.

In addition to answering people's immediate needs, some authors like to educate the questioner about related best practices, alternate solutions, more general lessons to be learned, and additional resources. For example, after answering a specific, highly contextualized question, one author included the following line: "For your information, I diagnosed your page using my diagnostic user style sheet. See [URL]. Try it yourself...” The value of this approach was discussed by one interviewee:

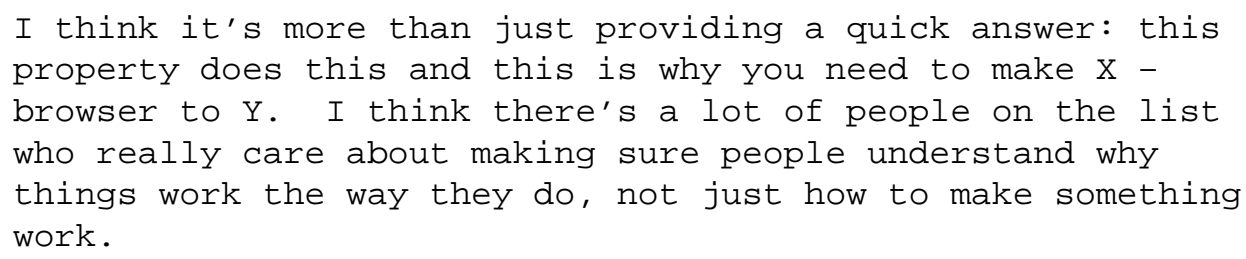
property does this and this is why you need to make $X$ browser to $Y$. I think there's a lot of people on the list who really care about making sure people understand why things work the way they do, not just how to make something work.

Question answering is occasionally a collaborative endeavor. Most questions receive at least two replies, and some of the more challenging or interesting ones can spur long discussions. Indeed, in the early days of css-d when the technology was new and many of the problems in implementing it had not been solved, the discussions ran longer. One list member, reminiscing about the "fun" early days, mentioned how members were “always experimenting and trying different things and having long intense discussions about the things we were discovering." Some of these discussions led to the discovery of now well-known CSS browser bugs or workarounds. 
Currently, collaboration in question answering is not generally around group problem solving, although it does happen on occasion as candidate explanations and solutions are debated and discussed. More generally, members fill in details that were missing from prior answers, post additional links to resources not mentioned, challenge or back up a particular answer, and clarify the potential problems or benefits of various proposed solutions. The question asker is also often part of this collaboration, as they provide additional relevant information - often at the request of list members. The following edited thread is typical:

Subject: Background Image Link

Sent by Mark

What I would like to do, but doubt I can, is make a background image an active link, so clicking anywhere in the image does its thing.

Reply \#1

\section{Sent by Kevin 5 minutes later}

You cannot do this with CSS. You could do it with HTML and JavaScript by creating a main layer on which everything else was placed and running a JS function when that layer is clicked.

[signature]

\section{Reply \#2}

\section{Sent by Stephanie 3 minutes after prior message}

[quote of original message content]

You might want to ask yourself, if this image needs to be a link, is it actually content? If so, it would be better to include it on the page via the <img> tag, not as a background image. Background images are just decoration.

If it really is decorative image that must be a link, you'll have to do some hacking/trickery. Such as, place a transparent image in the div with the background, size it the same as your bg, and make that image the link.

Steven

\section{Reply \#3}

Sent by Mark (again) 2 hours and 29 minutes after prior message

Purpose - this will be a substitute page, for a lot of individual pages that have a link to a list of the pages. 


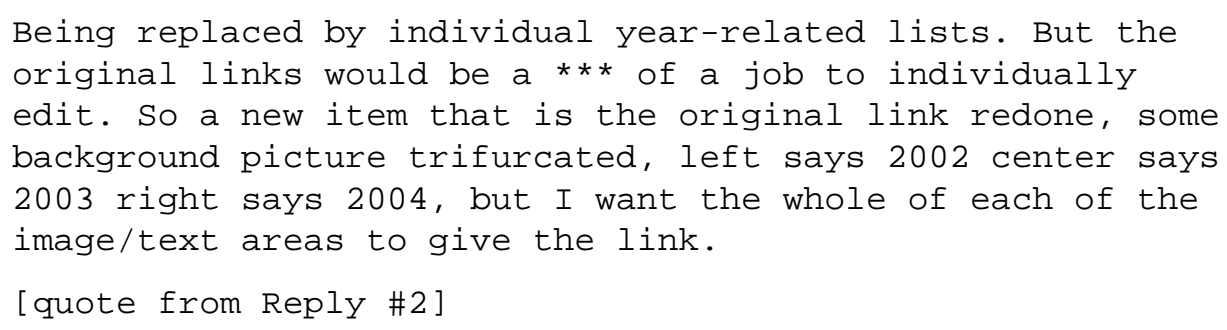

\section{Reply \#4}

Sent by Joseph 7 hours and 10 minutes after prior message Hello list,

$>$ What I would like to do, but doubt I can, is make a background image > an active link, so clicking anywhere in the image does its thing.

It's possible with $(x) h t m l$ and css, no Javascript:

html:

$<$ div $><$ a href="\#" title="\#" $><$ span $></$ span $>$ link text $</$ a $></$ div $>$ css:

[includes 16 lines of cSS code]

At least it's the classical IR [image replacement]. disadvantage: in IE-Versions, you won't get the cursor while hovering.

greetings

Joseph

This thread highlights the value of having an entire community involved in question answering. The first message represents the questioner's compromised need expressed as a question about the feasibility of a specific technique using CSS. The first reply answers the question directly (in the negative) and provides some very brief pointers to non-CSS techniques that may work. The second reply questions the appropriateness of the original question and attempts to get at the underlying need through a question to the questioner. It also suggests a CSS technique that may be appropriate, depending upon the underlying need. After this, the original questioner replies with a more complete description of the underlying need. Finally, another member replies by including a technique that works with a combination of (x)html and css. The example is presented as actual code, which is common at css-d. It also includes a caveat as to the limitations of the approach. As is also generally the case, there is no final 
message from the original questioner explaining his final decision. This makes it difficult for others to learn from his experience.

Occasionally, a discussion will ensue among answerers regarding the appropriateness of various, competing solutions. Thus, questions often provide a jumping off point for community discussion and debate, some of which may only loosely apply to the initial questioner's situation (see "Holy War” section below). Interviews and content analysis suggest that these debates were much more common during the first year of the list than more recent years. In recent years arguments about the appropriateness of various techniques are typically spoken in the language of the questioner's original need as in the thread shown above. However, question answerers often find ways to insert their own opinions about more philosophical issues and best practices as side comments when answering a question (e.g., “warning, opinions follows”; “...but I digress”, “but you really may want to consider the implications of...”).

In summary, the Q\&A genre system is composed of a question message followed by one or more reply messages. The majority of these messages include highly contextualized text based on the immediate need of the questioner, with frequent inclusion of code snippets and URLs. Most threads include at least 2 reply messages, with some being truly collaborative endeavors to answer a question effectively. The socially recognized (and "approved”) purpose of such threads is to satisfactorily meet the needs of the questioner, as well as further educate the questioner and other list members who read the thread. In meeting this social recognized purpose, members also find ways to share their opinions on related issues, although opinions without a practical value to the questioner (and to a lesser extent other members) are generally frowned upon by the administrators.

Site Checks 
Another common genre system is referred to by the community as Site Checks. The socially recognized purpose of a Site Check is to provide meaningful feedback on a website, as well as to further educate the requester of the Site Check and other list members. These threads begin when an individual solicits comments from other community members on a website that she has designed using CSS. Because the same CSS code is rendered differently by different browsers, Site Checks often include requests for members to view the site in a browser not immediately accessible to the poster. Reply messages provide comments on the site and are often sent directly to the individual, especially if they are about non-CSS issues. Site Check threads often include the words "site check," "site help," "browser check" or some derivation of those in the email subject line. Identifying them solely on this basis, they make up approximately 6 percent of all list messages, an average of about 3 per day.

\section{$\underline{\text { Site Check Requests }}$}

A typical Site Check request message is shown below:

\section{Subject: Site check - from Windows users, please Sent by Jessica}

Hi all,

I'm testing a layout for my website here: [URL included]

I've included a few options to change colours and fonts in the sidebar on the right. Screenshots of how the pages look on Mac OS $X$ in Safari are also linked from there.

If anyone on Windows systems (or any non-Mac systems basically, as Macs are all I have access to at the moment) could let me know if they notice anything out of place or significantly different from those screenshots, I'd be really grateful.

Don't pay attention to the text - also, colours in template 3 and 4 are still being worked on...

of course, if you also want to add which fonts/colour schemes you like best, or have any suggestions for improvement whatsoever, that'd be a plus :) but the positioning and alignment of elements is really what I need to check most. 
Thanks a million in advance!

Jessica

In this example, Jessica used a subject line indicating her need for a Site Check and the platform/browser she needed it checked in. Specific requests for browsers are common because designers often don't have all platforms available to them. As is also common, she provides some idea of what she would most like feedback on ("positioning and alignment of elements). Other Site Check requests include even more explicit questions (e.g., "could someone please test the new page to see if the bottom row of navigation works?”). In addition to specific needs, Jessica asks for feedback on non-CSS issues (“fonts/colour scheme”) and general "suggestions for improvement.” Administrators (and other members) allow these technically "off-topic” requests to be posted as part of a Site Check, although members are encouraged to reply directly to the sender if their comments do not relate directly to the CSS aspects of the page. Finally, as is generally the case, Jessica thanks members in advance for their comments.

The following Site Check request illustrates another common practice of justifying certain practices:

Subject: site check

Sent by David

thanks for the help previously on the print style sheet for this site, it looks great and my boss likes it. can I get a site check? Especially those with macs, but any check would rock. i've test in IE6 and firefox 0.8 .

[URL included]

$i$ have to use tables b/c $i$ am required to use a university template since it is a academic website. i don't like it, but $i$ 've tried to clean them up as much as $i$ can.

thanks for the help, david.

In this example David thanks others for their help in answering a question in a prior thread and indicates that the solution pleased his boss. He then requests a Site Check and specifies the desired platform and those that he has already tested. In the final 
paragraph David justifies his use of HTML tables to the community as an external requirement imposed upon him. The css-d community often chides people for designing websites that rely on HTML tables for positioning instead of pure CSS code. His justification of tables allows him to save face with the community and removes the need to educate him about the "evils" of positioning with tables. Other Site Check requests indicate unfinished sections of the layout, also in order to avoid unnecessary work by community members.

\section{$\underline{\text { Site Check Replies }}$}

Reply messages provide reactions to the specific requests made, as well as general comments about the overall visual appeal, usability, and use of CSS on the site. They are often sent quickly after the original replies, which is part of their value. For example, some members mention that they need feedback on a particular platform since they won't have access to it until the evening. I did not have access to messages sent outside of the list (probably the majority of them), but interviews suggest that they are similar in nature. The key difference is that those sent to the list are more focused on the CSS issues related to the site, since that is considered on-topic.

The following were replies sent to the list in response to the first Site Check request shown above:

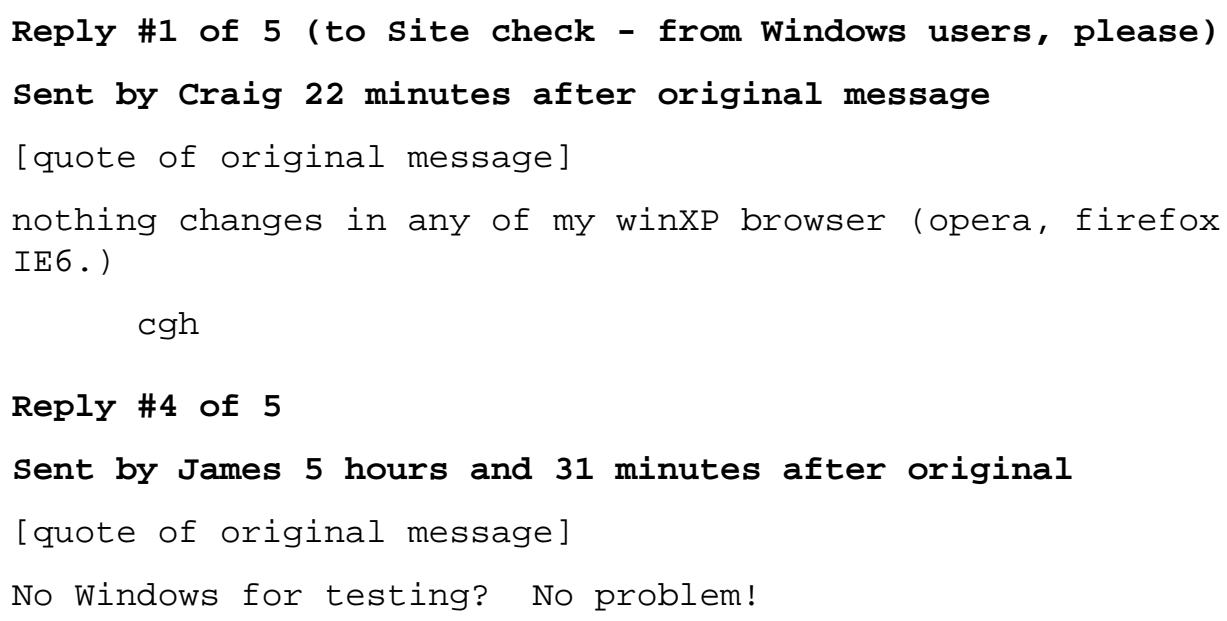


[URL to web tool provided] gives you screenshots in:

Internet Explorer 6.0

Internet Explorer 5.5

Internet Explorer 5.01

Internet Explorer 4.01

Mozilla Firefox 0.8

Opera 7.23

James

[signature]

These are examples of very brief replies with little personal commentary. The second one introduces a web design tool that creates screenshots of a page as they look using various browsers. This is a typical example of how knowledge about tools and resources are shared among list members in the context of a specific need. Replies \#2 and \#3 include comments and a follow up post from the original author and are not shown for brevity reasons. The final post in the thread is included below as an example of the details that are often provided.

Reply \#5 of 5

Sent by Peter 7 hours and 24 minutes after original

[quotes from original message and follow up message]

I've looked at your page in opera 7.50 , Firefox 0.8 and IE 5.0 on win2K-pro.

You've probably got it as consistent as anyone can make it, as the page holds well on screen-widths from 600 to 1600

px. Some might like the text a little larger to begin with, but none of my browsers breaks that page when resizing within the browsers, so I think it's fine at it is.

- Opera and Firefox can take that page down to a width of about 500px without creating a scrollbar, and it looks just fine.

- IE 5.0 is not centering the page, so it doesn't look the same there as in the other browsers. I wouldn't know if I couldn't compare, so the page isn't breaking or anything. It just leaves all the empty space at the right side, and breaks the page on smaller width than about 600px.

Maybe the "centering-method for IE-win" might be the thing here, but it looks good as it is too. 


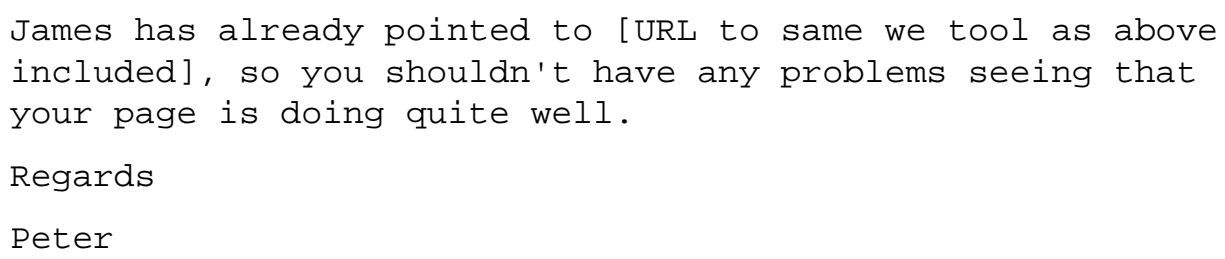

Like Q\&A discussions, Site Checks like this one are typically focused on the immediate needs and the language is highly contextualized to the particular situation. For example, Peter talks about the centering not working in IE 5.0 and the width at which Jessica's page needs a scrollbar. The result is that Jessica gets a personalized discussion about her site. She is also introduced to new relevant resources (e.g., the screen capture web tool) and potential techniques (e.g., the “centering-method for IE-win” technique briefly mentioned by Peter) that allow her to address some of her questions on her own later if desired.

One more thing to note from this example is Peter's response to the issue of text size that was brought up earlier in the thread (in the messages not shown here). Just as question answering can be a collaborative effort, so can Site Checks. However, this is only the case for messages that are sent to the entire list. Messages sent to individuals cannot be “peer reviewed” by other list members.

The following quotes taken from several Site Check responses highlight the fact that many techniques and best practices, as well as opinions are shared during Site Checks:

- I'd consider adding some :hover effects to your links. From an accessibility perspective, it's a good visual cue that you're correctly targeting the link, particularly for users with limited vision or mobility who might otherwise have trouble telling when they're able to click.

- Personal note: That whole "control the user's browser to meet my design needs" is a bit irritating.

- Never enclose URLs in single quotes. Use either double-quotes, or none at all. That will allow IE5/Mac to see all those backgrounds. 
Although these insights are raised in response to specific individual's websites, they are often of value to others. Several interviewees mentioned that they learned a great deal from Site Checks because they were able to see fresh designs, as well as overhear expert critiques of them. It can be a source of design inspiration, as well as a warning sign against common problems. While Q\&As generally address the known needs of website authors, Site Checks address the unknown needs that are recognized by experts but not the original designer. They also serve as fodder for discussion about best practices, common pitfalls, and novel approaches.

\section{Holy Wars}

The term Holy War, as applied to threaded discussions is not unique to css-d. Wikipedia defines it as "a drawn-out disagreement" where the "root of the disagreement is a clash of values” ("Flaming (Internet),"). The Netlingo Internet Dictionary defines it as "Perpetual BBS discussions that never die, the arguments never change, and no one's opinions ever budge one iota" ("Net Lingo: The Internet Dictionary," n.d.). These Holy Wars have the potential to lead to hostile interactions such as personal attacks (i.e., flaming), although they need not in order to be considered a Holy War. As one administrator explained in a message to the list, "Such discussions will at best clog up the list with fruitless back-and-forth, and at worst ignite a massive flame war. Neither is acceptable.” This explains why administrators and some members try their best to shut them down or nip them in the bud.

The Holy War genre system at css-d is a bit different than others discussed so far. Indeed, its status as a genre could be questioned because of its unique characteristics. Like other genres (and genre systems), it is recognized by the community as a particular type of communication with certain characteristics and elements of form. Unlike traditional communicative genres and genre systems (e.g., office memo, recruitment process), Holy Wars are not started in order to serve a particular socially recognized 
purpose (Orlikowski \& Yates, 1994). Instead, they typically are only identified as Holy Wars (or potential Holy Wars) in order to stop them from continuing (or prevent them from occurring. Like other communicative genres, the concept of a "Holy War" is useful as a "shorthand" that can make communication more effective (Orlikowski \& Yates, 1994). For example, when people refer to a thread as a Holy War, members understand the social implications and activities occurring within the thread - even without having to read it. However, unlike other communicative genres, Holy Wars do not improve communication efficiency - in fact, in many ways they are the antithesis of efficient communication. Although their classification as a genre system could be debated, I classify them as such because the concept of a Holy War is well defined and shared among css-d members and is useful as an analytical category that can be referred to later.

Holy Wars are relatively rare at css-d, although they are very salient to the community. It is difficult to quantify them since the specific words "holy war" are not always applied. A search for the words "holy war”, "holywar”, and "holy-war” revealed only 42 messages in a 32 month period (less than $0.01 \%$ of all messages). However, interviews revealed that members know about the common topics that lead to Holy Wars and even the common perpetrators.

Unlike the other genre systems discussed so far, Holy Wars rarely begin with the first message in a thread, although the first message may bring up the confrontational issue. Instead, they begin when a member of the community posts a statement of opinion and continue when others reply to the statement. This can happen as part of other genres as the following example shows:

Subject: Comments Requested on website.com Sent by Nathan

I have recently launched the *beta* version of my new blog at wWw.website.com. There are no graphics yet, but the layout is in place.

I'd like to get some public feedback on the display. Do you notice any browser-specific quirks? Is the text too small? 
I already know of two issues.

1) If using IE Win, with text set to "smaller," you can't read anything. This is because the font-size is set to $\odot .67 \mathrm{ems}$.

2) [non font-related issue discussed]

Special things you might want to look at:

1) [non font-related issue discussed]

2) [non font-related issue discussed with code snippet]

3) Being an accessibility kind-of-guy, you'll find that the text of the individual articles is in a larger font size. So you young whippersnappers with eagle sharp eyesight can read the tiny stuff, and those of us who are nearly blind from starting at computer screens for years can read the "larger-type" version. If you have any thoughts on this approach, which I think is rather unique because everyone else uses style-switchers, I'd like to hear them, although they may be better served for off-list.

This is a typical Site Check as described above. Nathan's request for feedback on the size of his text and his novel approach to sizing text (as part of this Site Check request) served as fodder for a holy war. In CSS there are a number of ways to specify the font size including using pixels, percentages, ems, keywords, and points. Each method has different implications for different browsers and user groups. In this case, the discussion degraded into a fruitless debate about whether or not users should be expected to change the browser's default text size settings. A few quotes taken from the 16 followup messages (all sent in less than 27 hours from the original) are typical of the Holy War genre:

Reply \#2 of 16

Sent by Jun

$>3$ ) Being an accessibility kind-of-guy, you'll find that the

$>$ text of the individual articles is in a larger font size.

Your stylesheet sets the body font-size to $0.667 \mathrm{em}$ ! That's only $2 / 3$ of the user's preferred font-size! Where's the larger font-size you promised?

Oh, I see! After I've squinted through the tiny text, I come to a red link that says "View this entry with comments". When I click on that, I finally get the normalsized version. Whew! 
OK, I exaggerated slightly to get my point across <g>.

If you really want to be more accessible, I'd suggest you

reverse the order: As a default, don't style the body text

at all; and as optional extra, provide the text in tiny

font-size.

Reply \#4 of 16

Sent by Nathan (original author)

OK, so I switched it over to $1 \mathrm{em}$ now. Ugh. Way too big for me. Now I've made it $0.8 \mathrm{ems}$. Ugh. Pixels are looking better and better in my mind. So now the text size is 11 pixels. Well, since most of the folks that I *hope* will visit this are members of various lists, and mostly savvy users, then I think that pixels will be my answer. Tough luck Win IE users.

Stupid browsers. I'd rather the browser makers agreed on font sizing and typographic issues than some other things.

Reply \#6 of 16

Sent by Jun (same author as Reply \#2)

If the default font-size is too big for you, then adjust your browser settings.

Hmm, I guess I'm thick. What does being a member of a list and/or or being web-savvy have to do with ridiculously small font-sizes?

After these messages 2 other members joined in and debated about whether or not users should be expected to change their default settings. The thread was finally shut down by an administrator with the following message:

Reply \#15 of 16

\section{Sent by Administrator}

If there one thing this list will never, ever make possible, it's a final resolution to the Font Sizing Holy War. This is why we established a Wiki page that gives people a good jumping-off point for reading up on various perspectives: [URL to FontSize page included]. The next time the war breaks out, let's just refer each other to that page and move on to discussions of a less religious nature. If there are non-font-sizing comments to make about website.com, let's get back to making those-- otherwise, I think it's time to move on. Thank you.

[signature]

This particular Holy War was started in Reply \#2, when Jun used sarcastic

remarks and strong language to discredit the approach taken by the Site Check requestor 
Nathan. It seems that Jun was partly reacting to Nathan's claim that he was an “accessibility kind-of-guy” (the line that was quoted by Jun in his reply). Although Nathan and Jun posted several of the messages, several other members joined the Holy War.

As happens on occasion, the Administrator shut down this Holy War. The details of his approach (i.e., referencing the wiki) will be discussed in Chapter 6. For our purposes it is enough to recognize that administrators do not like when Holy Wars continue and often try to shut them down. It is also common for members or administrators to mention their desire to steer clear of a Holy War when asking a question on a controversial topic like font sizing.

\section{Announcements}

Another somewhat frequent communicative genre is the Announcement message genre. The socially recognized purpose of this genre is to inform list members of new material. Although a few members identify messages as announcements (i.e., by using ANNC: in the subject header) it is rare. Announcements are about new articles posted about CSS (often by the list member authors), CSS or web-design related events (e.g., conferences), updates to CSS specifications, solutions to common CSS problems, major website redesigns using CSS, and new browser releases. The form is flexible, with some Announcement messages containing only a sentence with a link and others including extended commentary. A typical example is provided below:

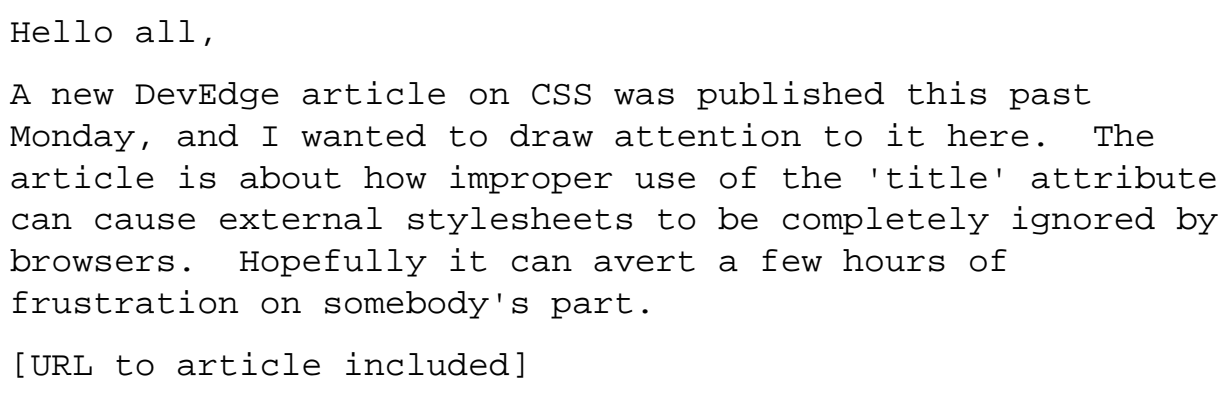


In general, new CSS-related content at DevEdge can always

be found at [URL included]. We're currently working to

make the site even more useful, so look for improvements in

the next couple of months.

This announcement was sent by a well-known CSS expert on the list. As is typical, this announcement includes a brief summary of the topic being announced and a link for more information. Announcements rarely include questions, although they do often lead to discussions about the content. Although these discussions, like all activity at css-d, are encouraged to stay on-topic, a bit of commentary typically follows. The following quote was taken from an announcement of a new website redesign and sent by an administrator who is modeling the type of behavior he hopes encourage: "I'm mentioning [the site redesign] mostly so anyone interested can go check out our work and the articles we wrote about it; I definitely don't want to hijack the list with a thread debating the pros and cons of our design choices.”

\section{Administrative}

Finally, there are a handful of messages that fall into the Administrative genre. Most of these messages are sent by the official list administrators, but a significant number are posted by other core community members. Those sent by official administrators often include the word "ADMIN:” in the subject line. The purpose of these messages is to enforce the governing rules outlined in this chapter such as keeping the discussion on-topic, friendly, and in line with list policies (e.g., no job solicitations or excessive quoting). In addition, some of them discuss technology-related issues with the email list when there are problems or changes. An example of an administrator shutting down a Holy War has already been shown. Another typical example, posted by an administrator, is shown below:

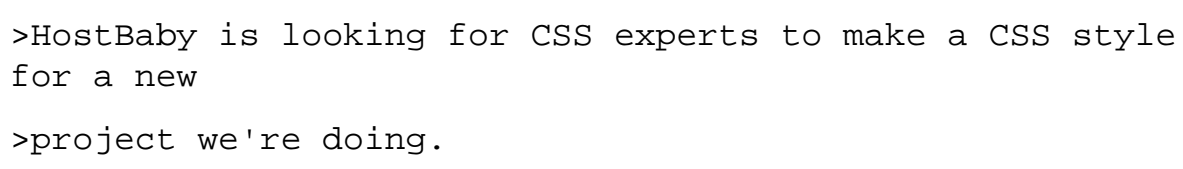




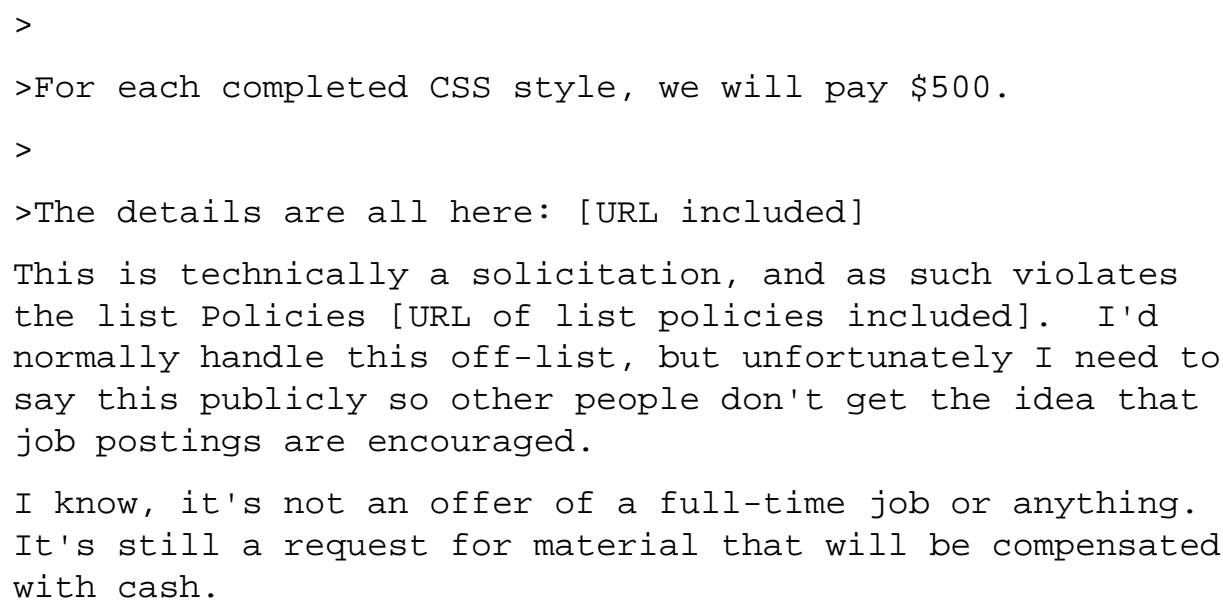

Notice that this message, like most Administrative messages was sent in reply to another message where there was some type of "misbehavior" occurring. Interviews of administrators revealed that many of these messages are sent in private - especially when they relate to private problems such as not trimming posts. When they deal with more public issues or the point needs to be re-emphasized to the community at large then they are replied to on the list as was the case here.

Administrative messages often include an appeal to the list rules and policies (see example above), the authority of the administrator, and sometimes arguments about the rationale for the policy. Most Administrative messages are short and to the point and they use strong language at times (e.g., “This has been said before, twice. Let's drop it people. If your post doesn't have anything to contribute to the practical uses of cascading style sheets, DO NOT POST IT! The thread is over.”). Some of them solicit a response by other community members who discuss the appropriateness of certain admin decisions, however such discussions are often encouraged to take place offline. Thus, the socially recognized purpose of this genre is to clarify list policies and curb "inappropriate” behavior.

\section{Participation and Community Roles}

\section{Email List Work}


As is typical in online technical support communities (Galegher et al., 1998; Hiltz \& Turoff, 1993; Lakhani \& von Hippel, 2003; M. Smith, 1999), participation in the email list is highly skewed. During our 28 month period, approximately 50\% of all messages were sent by the 157 most prolific providers (4\% of all providers; Gini coefficient of 0.75). The top poster accounted for $3.2 \%$ of all messages. A third of posters only started threads (generally by asking a question), $16 \%$ only replied to others' messages, and the remaining 53\% did both at least once. It is not possible to calculate the number of "lurkers" (subscribed members who do not post messages to the list) for the specified time period, but as of July 2006 there are over 7,500 members, far more members than the 3,894 individuals who have posted a message since the list's inception.

Although I have discussed the major shared activities (i.e., genres), there are a number of specific activities that happen within the constraints of those genres. As the prior examples show, members express gratitude (e.g., through "thank you”s), provide favors (e.g., Site Checks), show off their websites and CSS knowledge, and discuss best practices. They also rant about the poor support of CSS in browsers and reminisce about the days when it "took guts to go without tables.” However, they rarely discuss personal issues unrelated to website design. Several members also regularly use humor especially humor that includes references to website design and CSS.

There is also a great amount of work that occurs behind the scenes. As we saw in the prior examples, members validate other's sites; download, modify, and test their CSS code; and capture screenshots of a webpage in a particular browser. They diagnose problems that are difficult to identify (e.g., browser bugs) and help develop workarounds (i.e., hacks) to known browser bugs. They also find and share resources (typically online resources) such as CSS design templates and new hacks. Although some of this is solitary work, much of it is done for social reasons.

\section{Community Roles}


Members of css-d play a number of social roles. I have already discussed the role of administrator. Here I discuss a few other common roles, some of which have been identified in other studies of online technical support communities (e.g., Lakhani \& von Hippel, 2003; Zhang, Ackerman, \& Adamic, 2007). Specifically, I discuss core participants, intermittent participants, and newcomers/lurkers.

Core participants are active members who have known each other for a long time. Data show that 45 of the 50 most prolific posters have participated for over a year, and several of them have been around since the beginning of the list. Like core participants of other online technical support groups, those at css-d have a high level of expertise (Lakhani \& von Hippel, 2003; Zhang et al., 2007). Many of these members are known as CSS experts both within the community and outside it. In fact, several of these members collaboratively author articles for well-known website development outlets. Interviews revealed that they frequently communicate with one another outside of the list via email and phone. Core members are heavily involved in answering newcomer's questions, as well as discussing their own work and projects. They often advocate CSS to newer members, helping win them over to the cause. In fact, several of the current core members joined the list as relative novices and through their participation became more central community members with high levels of expertise and a desire to "give back" to the community. They also participate in the list to keep up on the latest CSS developments.

Intermittent members post less often than core members, although many of them have been a part of the community for a long time. For example, the median number of posts for the 474 members who have participated for over a year is 19, suggesting that there are a significant number of long-term, intermittent members. Interviews suggest that some of them read all of the messages in order to learn from them, although they rarely post, while others only read and post messages when they have a pressing question or need feedback on their newly developed site. Their level of expertise also varies 
considerably. Some of them have considerable expertise in non-CSS website design technologies (e.g., scripting languages) and participate when questions related to them come up. Although these members may crack jokes and share their excitement over new discoveries, they are less likely to communicate frequently with other list members outside of the list.

Newcomers are the least active members with the lowest level of CSS expertise. Most of them ask a single question and then leave, although some of them stick around and hide in the shadows as lurkers. They are primarily concerned with getting answers to their own immediate questions and are far more likely to ask questions than answer them. Although they do not reveal strong relationships with other members, many of them frequently reference the core members by name suggesting that they are familiar with the community. It is also common for these members to include social niceties and expressions of appreciation, perhaps in hopes that their questions will be replied to.

\section{Reuse of the Discussion Archives}

The community discussion is read by a very large number of non-community members through the public archive. For example, in July 2006 there were approximately 190,000 unique visitors with nearly 790,000 page visits. In other words, 25 times more individuals accessed the discussion as a secondary resource than there were members of the list. Visitors came to the site primarily from search engines (71\%), sometimes from bookmarks or direct access (26\%), and rarely from external web sites (3\%). This pattern

has been stable for the entire time period for which there is data (October 2004 to June 2007). Although most visits (76\%) lasted less than 30 seconds, there were over 10,000 visits that stuck around for over 30 minutes. Tellingly, over 66,000 of the 77,669 messages in the archive (i.e., 89\% of all css-d messages) were accessed during July 
$2006^{3}$. The 1,000 most frequently accessed messages were just as likely to be old messages as new ones. For example, the median message rank (with 1 being the oldest message) was 39,921 , which is close to 38,835 - the expected median rank of a randomly selected subset of the entire corpus based on a uniform distribution (i.e., half of total number of messages in the corpus). Likewise, the $25^{\text {th }}$ percentile and $75^{\text {th }}$ percentile rank messages (23,709 and 53,753 respectively) are similar to their expected values (19,167 and 57,501$)$. So many different messages are accessed because such a variety of search phrases ultimately lead to the archive messages. For example, over 150,000 unique search phrases (using 17,000 unique keywords) were used in July 2006, a month with 300,000 unique page visits from people coming from search engines.

\section{Conclusions}

This chapter has introduced the css-d email list community’s technical infrastructure, governance policies, primary genres and activities, participation patterns, and social roles. The focus has been on how those activities occur and the implications of them for information sharing and reuse of information within css-d.

Several important conclusions can be drawn. The first key insight is that the most common use of the email list discussion is its reuse by lurkers and non-members who search the public archives. However, the content that is created in the email list is not typically created with reuse in mind. What's more, many of the things that make the email list most useful to its members are the same things that make its reuse problematic. These are summarized in the following paragraphs.

${ }^{3}$ This number is based on the reports produced by Advanced Web Statistics 6.5 software package (Destailleur). It is supposed to have removed the effect of search bots, but because the raw data was not available, I was not able to verify this. Thus, it is possible that the percentage of unique messages visited via a search engine is overstated. However, the fact that there were 150,000 unique search phrases suggests that the numbers are reasonable. 
The email list is very good at providing personalized answers to questions or feedback on web sites. In the words of one interviewee, the unique value of css-d comes from the fact that "someone’s listening” and is “willing to answer.” Answers to questions at css-d are customized to the questioner's specific needs and even customized feedback is given in Site Checks. This can be extremely helpful, especially when inexperienced individuals are trying to do difficult things like diagnose a problem or implement a complex technique. However, this personalization of information makes reuse of the content difficult, since other readers' situations are rarely exactly the same. Furthermore, it takes considerable expertise to de-contextualize and re-contextualize the content, expertise that many archive readers and lurkers may lack.

The email list is a social place where friendships are developed, favors are provided (e.g., Site Checks), appreciation is expressed, jokes are laughed at, and topics people care about are debated. While this can lead to some social challenges such as Holy Wars and negotiating the boundary of on and off-topic discussion, its social nature is what ultimately makes it an enjoyable place to hang out. It is what makes it a community, not just a help desk. However, again we see that the very same social activity that is so necessary for the community to exist makes reuse of information problematic. When people come to the archives searching for an answer to a question, they must weed through all of the social niceties, fruitless debates, and "thank you"s. If this were simply a matter of ignoring the "social content" it would be annoying but straightforward. However, the information content is not entirely separate from the "social content," rather it is shaped by the social context in which it is created. The result is that it sometimes requires knowledge of the social context to extract the information content accurately. Understanding that a particular comment was written as part of a Holy War or that the person who said it is known on the list as an extremist may be important in knowing how and when to apply it. 
The immediacy of the email list is also a core strength. As we saw, people post Site Check requests while at work so they can get help before returning home. Questions are often answered within minutes, or at the very worst, hours. Announcements are sent to the list as soon as they hit the press and sometimes before. Email, with its push technology, is well equipped to support this immediacy. The quick pace allows members to post messages without having to decide if what they are posting is of "lasting value" or not. This is helpful to members because they are living in the moment. They can post guesses at solutions or half-baked ideas because they know others have the chance to react to it. However, this can be harmful for those searching through the archive after-thefact because the archive includes things like bad guesses at solutions, outdated announcements, and Site Checks with now-defunct URLs. The analysis of the email archive suggests that many of these problematic messages serve as entry points to the discussion, since nearly all messages were visited at least once when arriving from a search engine.

The inherent challenges of reusing the existing conversation help us see the wisdom of the css-d administrators' careful balance between encouraging the reuse of prior conversation and recognizing its limitations. List members are encouraged to find answers to their own questions in the archive (or wiki) if they can, but the inherent challenges are well recognized and so members are repeatedly told that "there are no stupid questions.” Too much emphasis on sending people to the archives (or wiki) can result in disgruntled newcomers, while no emphasis at all can result in an added burden on long-time list members who must deal with repeat questions. The practice of answering basic questions with a reference to a helpful resource (including the archives or wiki), while still contextualizing that resource, is an important one that has worked well at css-d.

Although I have highlighted the challenges of reusing conversation in this chapter, it is important to recognize the value of archived conversation. As we saw, a vast 
number of people continue to use the css-d email archive. Thousands of people return to it, often through bookmarks, suggesting that they continue to learn from it. It is clearly a resource of great value, even if that value is difficult for everyone to extract. Interviews suggest that this value comes from the fact that it is so comprehensive compared to other resources. Furthermore, it benefits from the fact that the topics of discussion are driven by the real-world needs of the everyman (i.e., the novice), while the substantive discussion is provided by experts. Thus, the challenge is to transform the conversation into a more reusable information object without losing its value or disrupting its flow. The css- $\mathrm{d}$ wiki discussed in the next two chapters is designed to address that very challenge. 


\section{CHAPTER 5}

\section{CSS-D WIKI REPOSITORY}

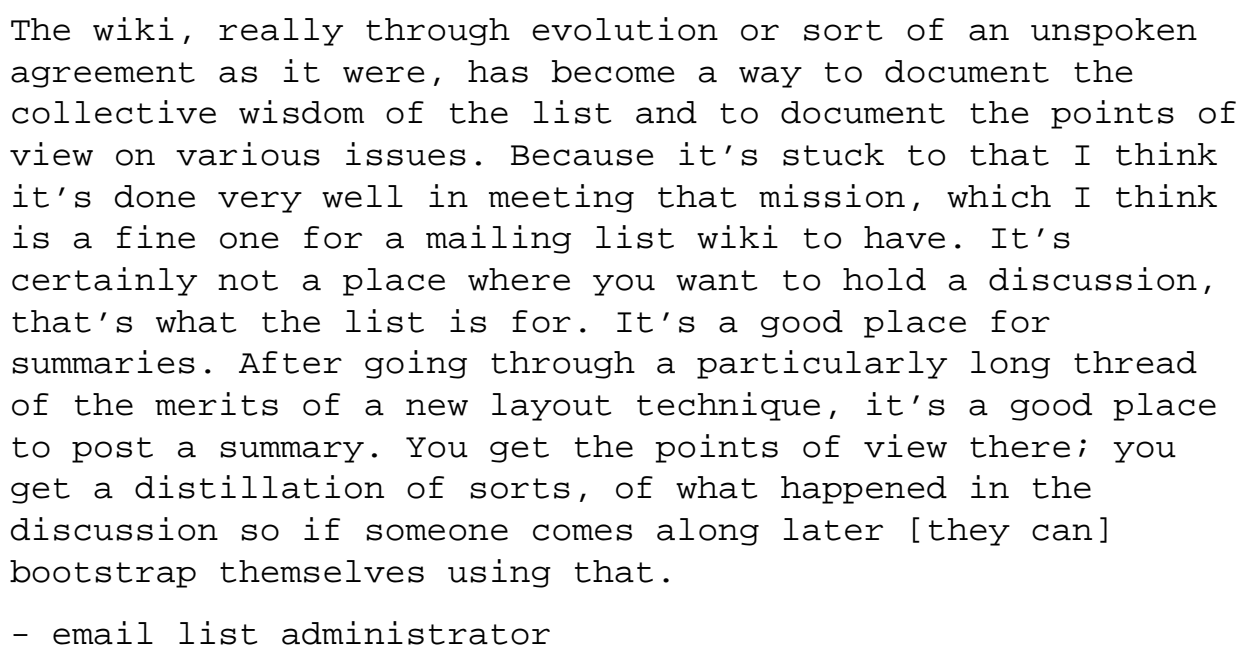

The css-d wiki is an important supplement to the email list, as well as a resource of more general interest. It is not technically linked to the email list (e.g., no shared membership information), but is closely coupled with it socially. Many of the same individuals contribute to both, the contents of each one refer to the other, and readers of the email list are expected to have looked for answers in the wiki as described in the previous chapter. The purpose of this chapter is to provide a closer look at the primary activities and social roles important to the creation and maintenance of the wiki, as well as provide an idea of the types of content that populate it and how they relate to other CSS resources. At the end I discuss some implications for design. The chapter includes the following sections which mirror those of the prior chapter:

- History

- Technical Infrastructure

- Governance 
- Genres and Activities

- Participation and Community Roles

- Conclusions

\section{History}

The wiki was created by a list member in August 2002, eight months after the lists inception (see Appendix 1 for timeline). The same member created the public archives around the same time. As discussed in Chapter 4 (Dealing with Frequently Asked Questions section), the need for an FAQ or wiki repository was brought up before this time period, but not initially acted upon. Interviews suggest that those who created the wiki did so in order to help document (and make accessible) the new CSS techniques they were developing (such as new browser hacks), help answer frequently asked questions, and help summarize Holy War arguments so fights wouldn't break out on the list. The quote at the beginning of this chapter, by one of the administrators who gave his approval for the wiki argues that the wiki has been successful because it has stuck to this initial vision.

Once the wiki technology was installed, a team of 5-6 volunteers helped seed the wiki with content before introducing it to the community. These members included the list chaperone, a couple of CSS experts who were active list members, and two people who were familiar with wikis but not as knowledgeable about CSS. They believed seeding the wiki with high quality content was important in motivating future high quality contributions as indicated by the following interview quote by one of the seeders:

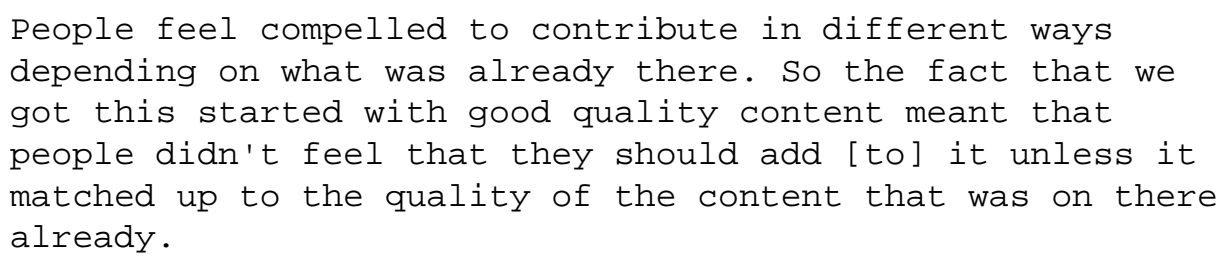


One member of the community believed this approach worked "extremely well" and another one suggested that the wiki has largely succeeded because it followed the pattern originally laid out.

The seeding of the wiki took approximately 1 month, after which time the wiki was introduced to the community. Other community members contributed for another month, after which time the wiki was introduced to the public (see Appendix 1 for timeline).

The wiki has shown moderate and consistent growth over time as shown in Figure 5.1, which summarizes data captured during several snapshots in time (see Chapter 3 for a discussion of the dataset). The total amount of content increased from $267 \mathrm{~KB}$ on April 21, 2003 to $750 \mathrm{~KB}$ on May 30, 2006 (an increase of 181\%). This represents an average of 437 Bytes per day or about 65 words. During this same time period, the total number of wiki pages increased from 220 to 546 (an increase of 148\%), representing an average of 2 new pages per week. Both the number of new pages and new Bytes decreased significantly around January 2005, although the exact reason is not known. More recently (i.e., between January 2005 and May 2006), about 1 new page per week and 290 Bytes per day are added. Only a handful of pages have been deleted. For example, only 5 of the 220 pages available in April 2003 were deleted by May 30, 2006. 
Figure 5.1: css-d Wiki Content Over Time

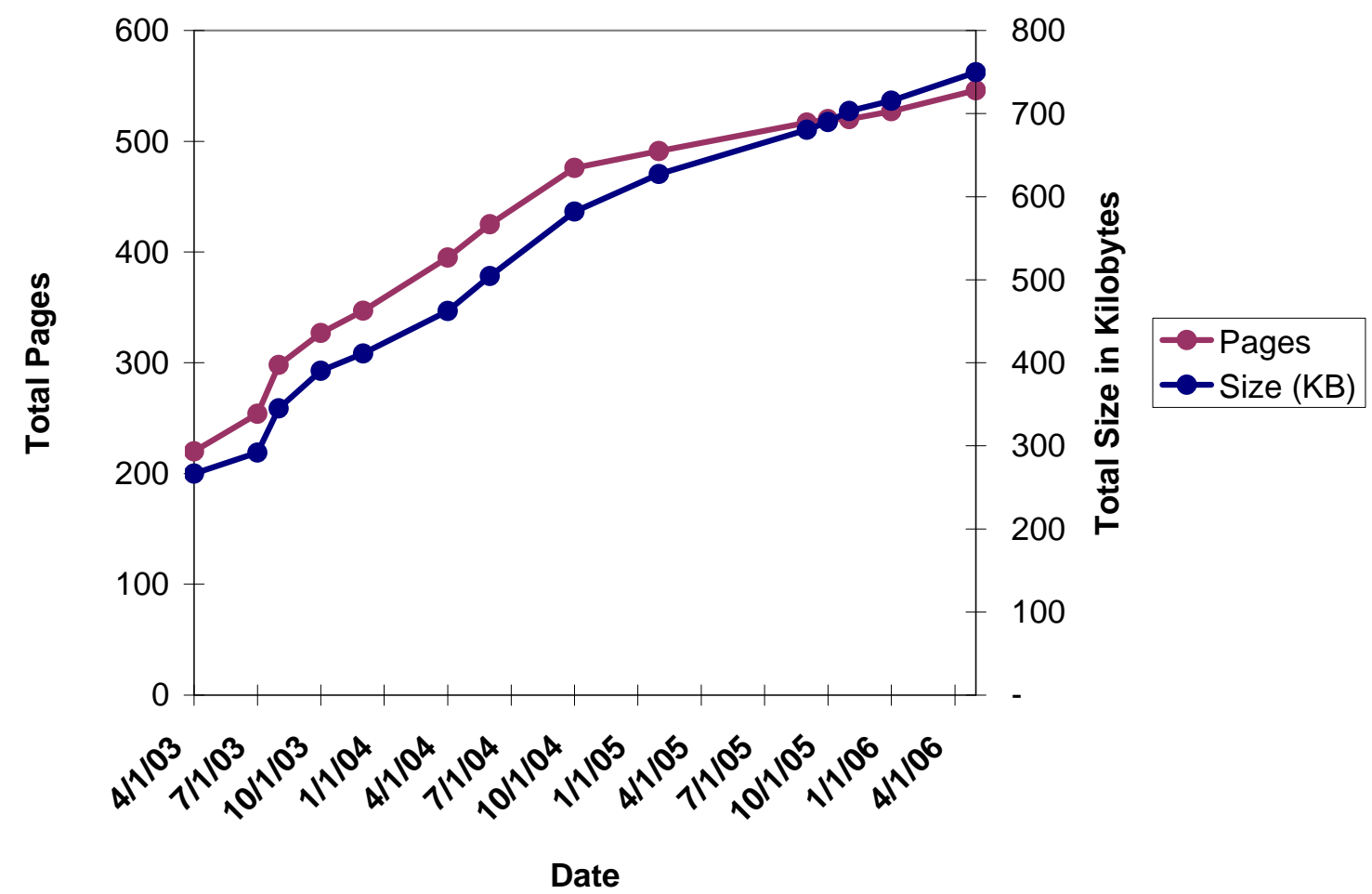

The use of the wiki has also increased over time. Community members have referred to the wiki in the email list more often over time (see Figure 6.1). Likewise, more individuals have accessed the wiki site over time as shown in Figure 5.2., which is based on wiki log server report data. For example, in June 2004 (the earliest time period for which I have data) there were 13,572 unique visitors that accessed wiki pages 124,805 times. These numbers have increased to 79,764 unique visitors (almost 6 times more) who accessed wiki pages 339,128 times (2.7 times more) in January 2007. 
Figure 5.2: Unique css-d Wiki Visitors Over Time

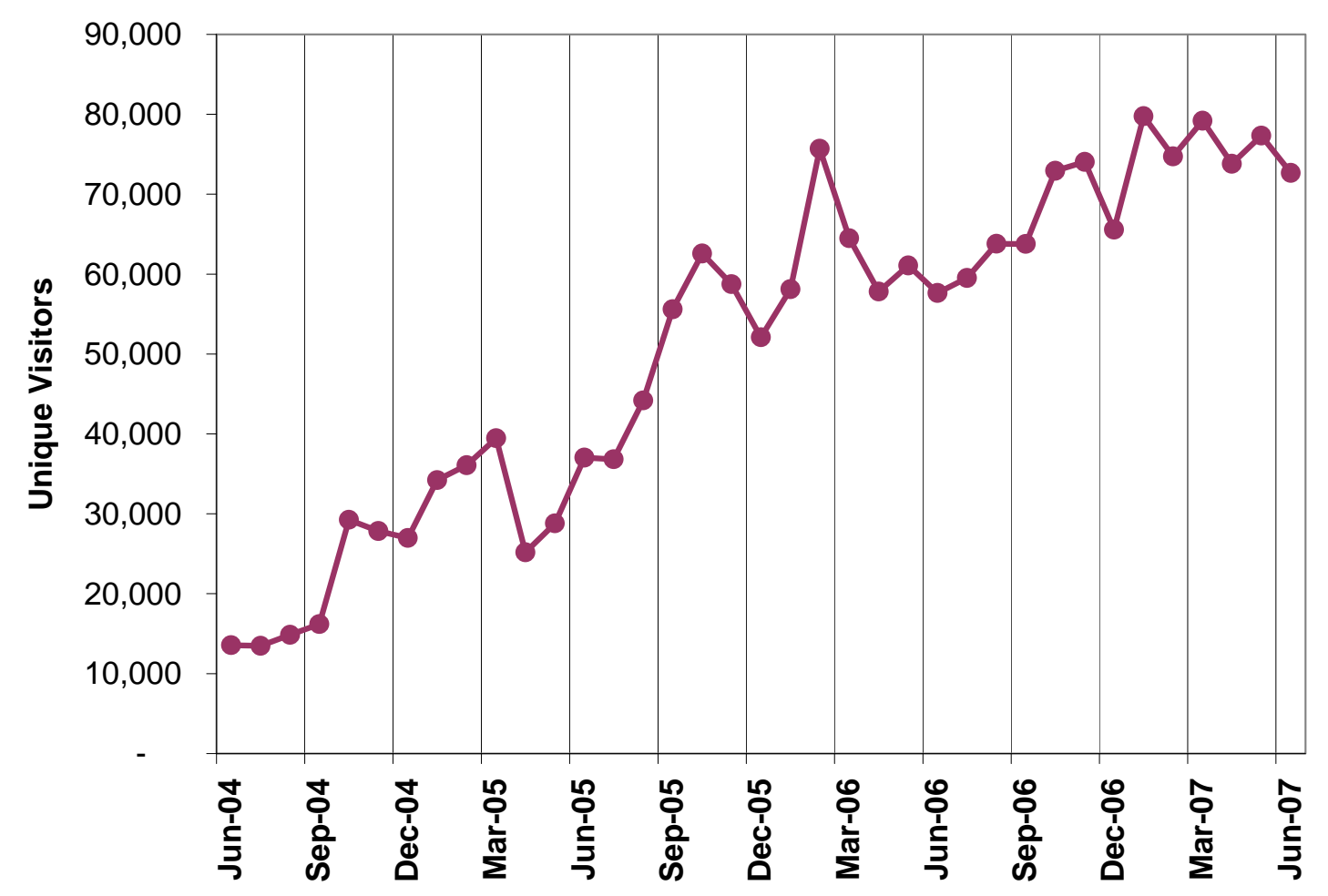

The increase in unique visitors comes from the fact that more visitors are referred to the site from search engines and other websites. The number of times visitors come to the wiki via bookmarks or directly (e.g., by clicking on a link to the wiki in an email client) has remained relatively constant over time. This suggests that the wiki was originally (and continues to be) an important resource to community members and regular visitors, while over time it has become increasingly used by non-community members who happen upon it through search engines and links from other websites.

\section{Technical Infrastructure}

A wiki is a collaborative authoring tool, or simply put, "a website that allows visitors to add, remove, and edit content" ("Wiki," n.d.). There are several different wiki software packages known as wiki “engines.” The css-d wiki uses the WikkiTikkiTavi 
wiki engine, also called Tavi wiki ("WikkiTikkiTavi," n.d.). The core features are outlined below, as well as some comments about how they are used at css-d.

\section{Wiki Pages}

A wiki consists of a network of specific wiki pages. A typical page is shown in Figure 5.3. Each wiki page has a unique URL that includes the title of the wiki page. For example, the URL for the page shown in Figure 5.3 ends with the page title: UnderstandingWiki. Hyperlinks are used to connect wiki pages together. Wiki pages are editable by visitors. To edit a page, a visitor can click on an "Edit this document” link at the bottom of any page (see Figure 5.3). This will take the visitor to a different view of the page (see Figure 5.4) that allows him to make changes to the text. Prior versions of pages are stored (and accessible to all visitors) for a two week period by clicking on the "View document history" link at the bottom of each page. This allows people to "revert" (i.e., undo) edits, for example to remove spam that someone has posted to a page or fix an inadvertent mistake. New wiki pages can be created by any visitor by linking to a nonexistent wiki page (see section below for details), following the link, and then editing the page as you normally would. 


\section{Figure 5.3: UnderstandingWiki Page}

\section{Getting Started}

- WikiGuide - an overview of how and why wikis work

- Wiki:WhyWikiWorks

- How ToCreateAPage, How ToEditAPage - a (hopefully) simple explanation of how to make a contribution.

- Editing Tips - the quick reference for markup used by this wiki. Answers how do I make text strong or emphasized or ...? - MultilineMonospace - carrying monospaced text over more than one line

- SandBox - where you go to experiment with wiki markup

- ToWikiOrNotToWiki - don't know whether to post to CssDiscussList or to the wiki?

Making contributions

- SignedAdditions - how to sign your comments

- ThreadMode - conversation style editing is easy

- DocumentMode - formal document styles can be more easily understood Getting along with the community

- CssWikiCoreGoals?

- WikiCulture

- RevisingWritingofothers

- ContentFolding?

Organizing Content

- CategoryCategory - The root of all categories used to organize pages into useful topics.

- FrontPage - The starting point for most people, giving visibility to important topics affecting the largest audience.

Edit this document | View document history

Document last modified Sun, 07 Dec 2003 23:01:05

Search:

In addition to regular content pages like the one shown in Figure 5.3, the wiki also has some dynamically generated pages that provide statistics. Several of these are linked to from the FrontPage (i.e., the wiki’s home page) including: PageSize, LinkTable, OrphanedPages, WantedPages, PageLinks and PageRefs. These pages can be used to find pages that are not linked to by other pages (OrphanedPages), pages without content that have been linked to from other pages (WantedPages), and various page statistics related to size, number of links on a page, and number of pages that link to a given page. Some wiki communities regularly use the OrphanedPages and WantedPages to help maintain orderliness on the wiki. It does not appear that users of the css-d wiki use these pages regularly because of the large number of pages on each of them. For example, the OrphanedPages includes one third of all wiki pages (169 of the 546 total wiki pages) and there are 201 pages that are linked to that have no content (i.e., WantedPages). Most of the pages currently listed as orphaned or wanted have been there for over a year. Also, no 
members volunteered any information about using these pages when interviewed and asked about their typical use of the wiki.

\section{Wiki Syntax}

Each wiki page can include structured elements (e.g., tables, bullets), hyperlinks, and formatting (e.g., headers, bold, italics). These elements are controlled by a markup language that is intended to be easier to use than HTML. For example, a “*” character at the beginning of a line followed by a space and some text will insert a bullet before the text when the page is displayed (compare Figures 5.3 and 5.4). Multiple equal signs surrounding a text string make the text string a header (e.g., see the "Getting Started" header at the top of Figures 5.3 and 5.4). Hyperlinks to other pages within the wiki are automatically created when the page title includes two capitalized words without a space between them (e.g., "WikiGuide” or "RevisingWritingOfOthers”). Linking to other

websites can be accomplished by surrounding the URL followed by a description of it in square brackets. For example, the wiki syntax “[http://abc.com ABC’s homepage]” would show up as [ABC’s homepage] and would take you to http://abc.com if selected. Finally, wiki syntax can be used to insert images, but is almost never used in this way at cSs-d. 


\section{Figure 5.4: UnderstandingWiki Page (Edit View Excerpt)}

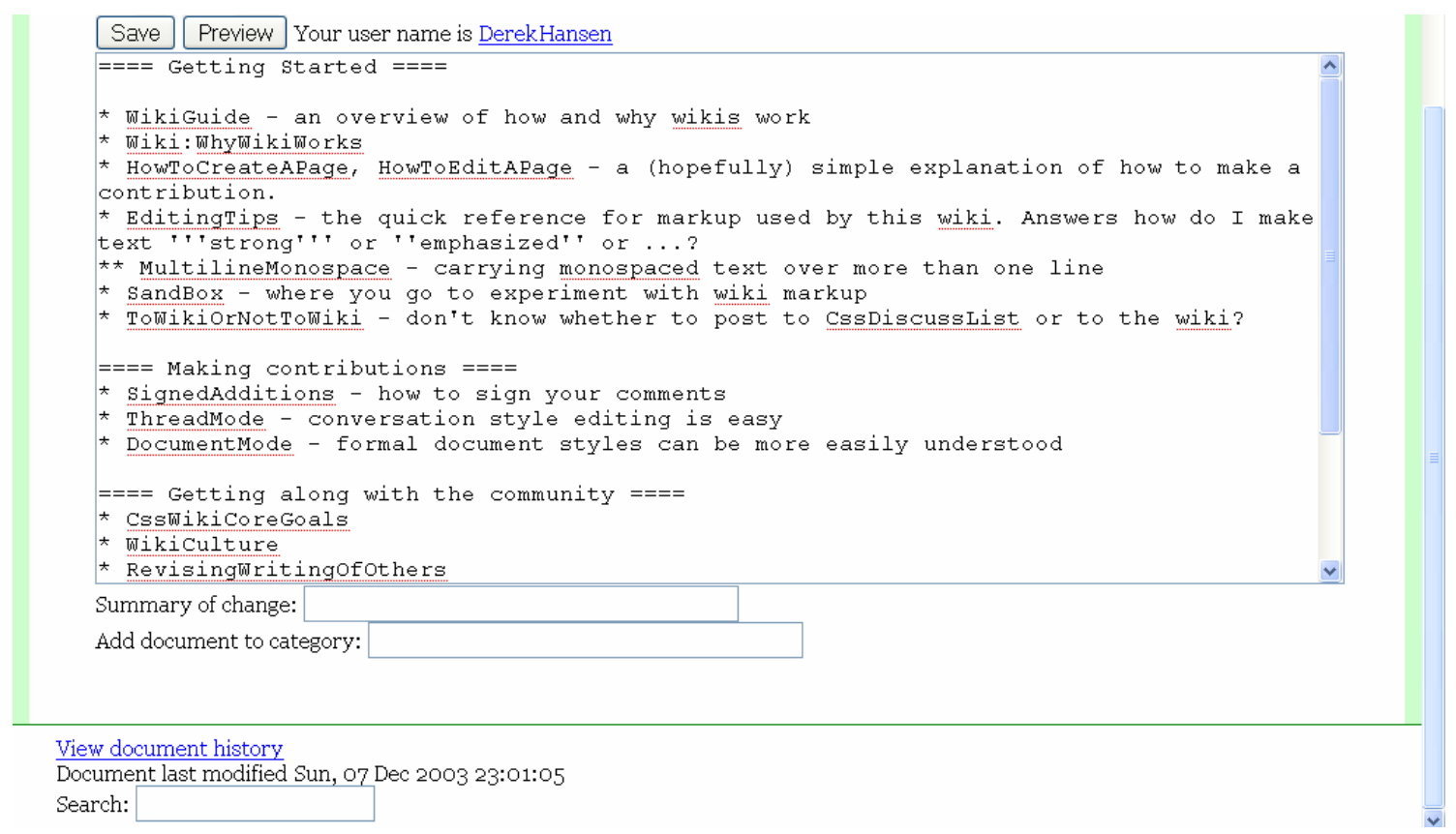

\section{Navigation}

Visitors to the wiki can navigate through it in several ways. One method is to follow hyperlinks from one page to another. Some pages, such as the FrontPage (the site’s homepage) serve as a manually created index to many other wiki pages (see Figure 5.5), while other pages include hyperlinks to pages from within a paragraph or in a special "Related Pages" section of the page. 


\section{Figure 5.5: FrontPage (Excerpt)}

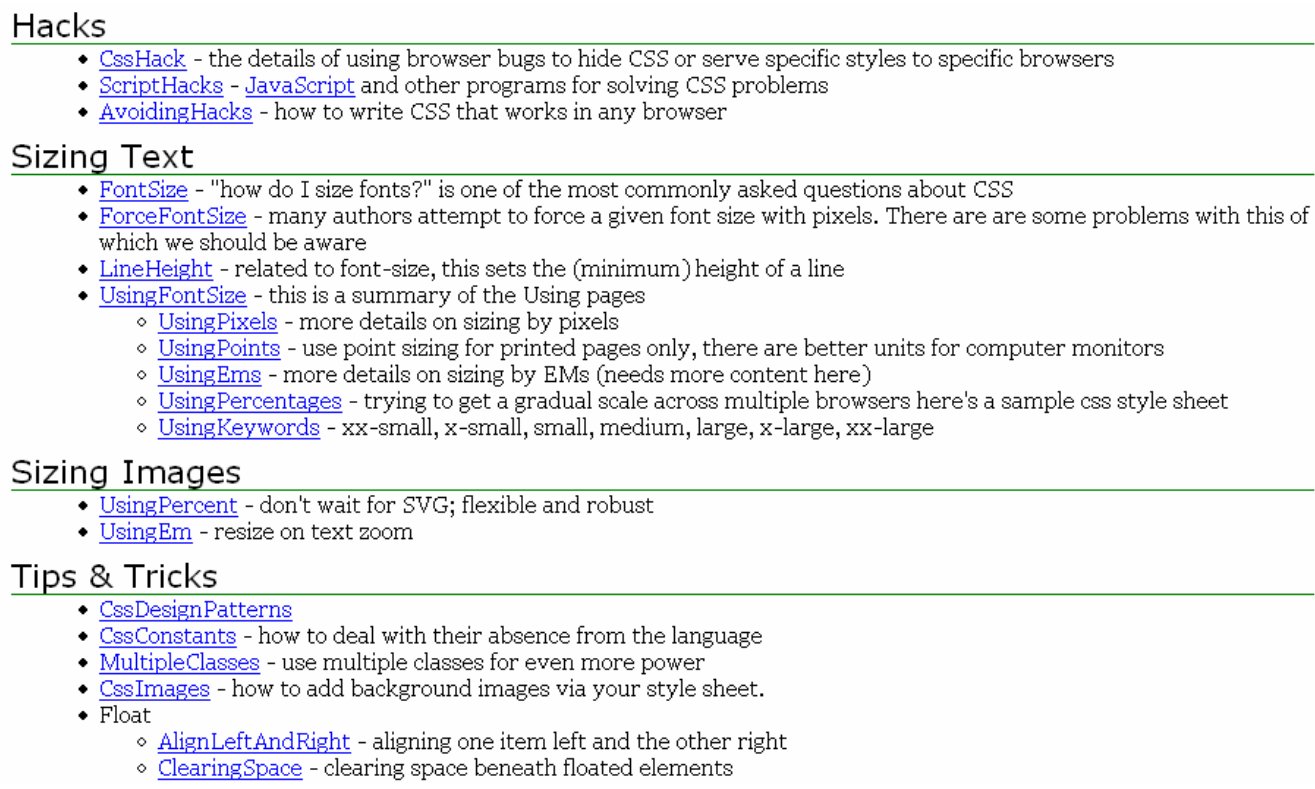

Another way to navigate through the site is to perform a keyword search using the search box at the bottom of each page (see Figures 5.3 and 5.4). When a search phrase (e.g., "background image”) is entered into the search box and the "Enter” key is pressed, a dynamically generated wiki page listing the titles of all wiki pages that include the search phrase is displayed. The search is not case dependent. When multiple words are included in the search phrase, the search treats the entire phrase (including the spaces between words) as a single text string and looks for exact matches. This is comparable to using double quotes around a search phrase on Google.

Members can also click on the title of a particular wiki page and be taken to a list of all pages that reference that page. This is called a backlink. A handful of pages rely on this feature to create special "Category" pages that list all of the pages in a given category. For this to work, someone must insert a link to the relevant Category page (e.g., CategoryBrowser) on each of the pages that should be part of that category (e.g., FirefoxBugs, OperaSix, MsIEsix). Then, when a person visits the Category page (e.g., CategoryBrowser) and clicks on the title, they will be shown all of the pages within that category. This practice was imported from other wikis and has not been consistently 
implemented or used at css-d. For example, the CategoryHomePage includes only $35 \%$ of all user homepages (i.e., Biography pages) identified through our manual coding. Likewise, the CategoryBrowser page does not include any of the recently created browser pages such as IE7. Only 2 of the 11 category pages are linked to from the FrontPage, which is the main directory of important pages. This is likely because of confusion about how to use category pages. For example, some members try to use category pages as indexes themselves rather than linking to them and using the backlink feature.

Finally, some people use the RecentChanges page to find pages of interest as described in the following section.

\section{RecentChanges Page}

The RecentChanges page (see Figure 5.6) is intended to help people keep track of edits to the wiki. All other wiki pages link to it (e.g., see Figure 5.3 directly under title). It displays information about the most recent edit of each page, sorted in reversechronological order. For example, the first line of the list shows the time of the particular edit (2006.12.05 22:02:01), provides a link to the page’s history where members can see exactly what was changed, a link to the most recent version of the page (BoxModelHack), and information about who made the edit (Figure 5.6). When a member makes an edit after logging into the site (or returning to the site with cookies enabled), the member’s username is shown (e.g., “John Smith” or “JanetteHansen”). When they are not logged in, their IP address is displayed instead. After the editor information, there is an optionally provided description of the edit shown in square brackets and bolded (e.g., “[Update]” or "[fixed defacement]”). These come from comments that people type into the "Summary of change:" box after making an edit to a page (see bottom of Figure 5.4). Finally, visitors can subscribe to the RSS feed for the page in order to receive notification when new edits are made. A few individuals mentioned using this feature to help hunt down wiki spam after a well respected member 
solicited help on his personal blog. However, none of the 6 members I asked during interviews had subscribed to the RSS feed, even though they were all active wiki participants. Despite this fact, these and other interviewees did mention regularly viewing the RecentChanges page to keep up on recent activity.

\section{Figure 5.6: RecentChanges Page (Excerpt)}

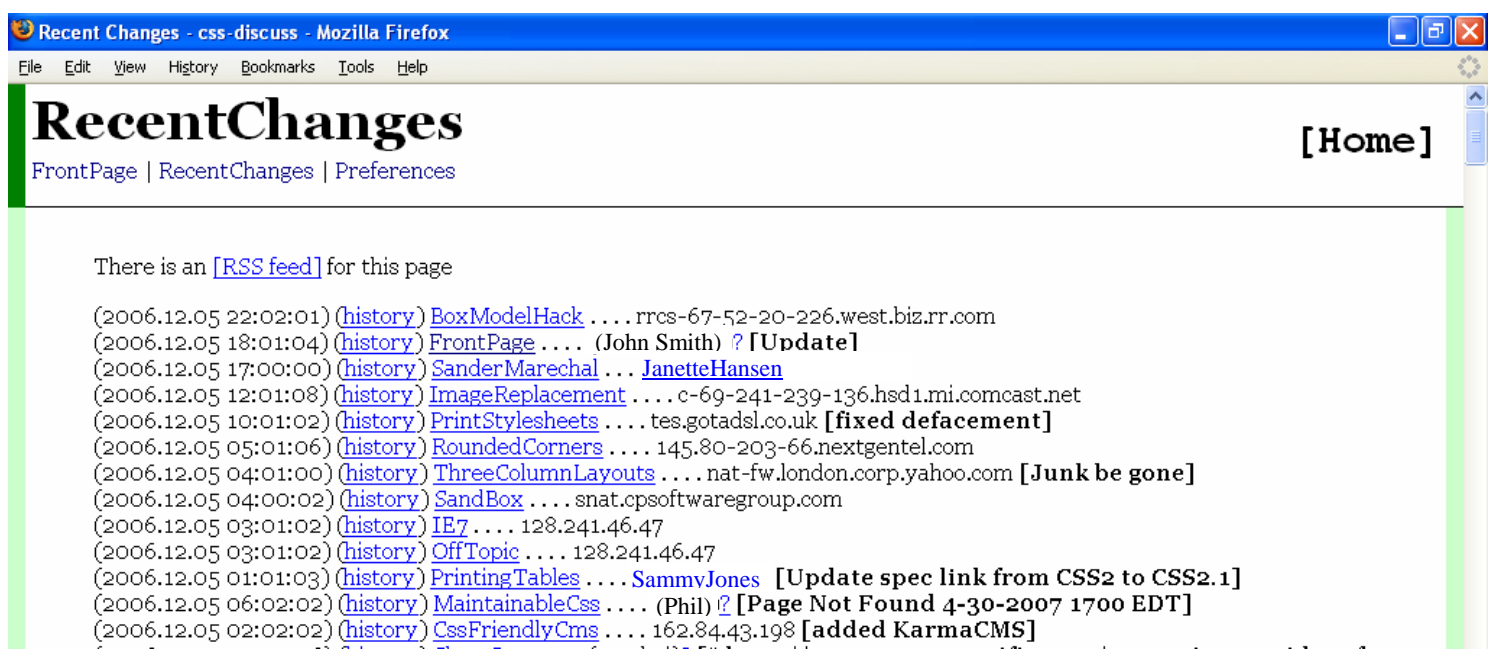

\section{Preferences}

Members have control over a few preferences, which they can change by following the "Preferences" link at the top of each page (see Figure 5.3). There is a space for a member to optionally include his name, which will be shown on the RecentChanges page as discussed previously. Most of the edits shown on the RecentChanges page only show IP addresses, suggesting that people either don't bother to edit their preferences or want to post anonymously for other reasons. The preferences page also allows users to control the size of the edit box (e.g., see Figure 5.4), the number of days displayed on the RecentChanges page (Figure 5.6), and the number of edits shown on individual page histories. 


\section{Spam Protection}

Many wikis, including css-d, struggle with spam that is added by anonymous users or spambots. This has been a continuous problem at css-d, as evidenced by several calls for help in removing spam, the creation of a WikiSpam page where members brainstormed ideas on how to fight spam, and the many summary comments describing edits as "spam removal.” One approach used at css-d is to block IP addresses of known spammers. Even after implementing this approach the amount of spam continued to increase. In 2004, at the request of a member, a simple password was required to edit. A hint was given so that members reading the message would know to enter in "css" as the password, but spambots and non-native English speakers would not. This was later replaced with a Tavi plugin that requires page editors to complete a challenge-response test before saving their edits (i.e., recognize some ASCII patterns as letters). However, even with these practices in place there has continued to be spam. Because these technical solutions are not perfect, individuals have monitored the wiki and removed spam manually.

\section{Governance}

The rules and policies associated with the wiki are not as well defined and enforced as those related to the email list. This is not indicative of the technology itself; rather it is a result of the way that this particular community has used the technology. Unlike some other wikis (e.g., WikiWikiWeb, Wikipedia, MeatballWiki), relatively little social interaction occurs through the css-d wiki since most of it occurs on the email list. This results in less of a need for administrator participation and policies/norms detailing appropriate behavior than is seen in Wikipedia (Bryant, Forte, \& Bruckman, 2005) or the WikiWikiWeb (Leuf \& Cunningham, 2001). 
There are no wiki administrators at css-d. A few individuals have defined a set of policies and practices related to the wiki on several pages within the wiki. However, they are not regularly enforced. This is likely due to the fact that so many people edit pages anonymously making it hard to know how to reach those that violate norms or policies. Most of the recommended policies were imported from the WikiWikiWeb wiki (Cunningham, n.d.), although interviews revealed that members also brought ideas in from other wiki communities including MeatballWiki, Wikipedia, and Twiki.

\section{Wiki Policies}

The wiki policies and suggested practices are spread out across several wiki pages. First time members are encouraged to read the WikiGuide page, which is linked to at the top of the wiki's home page (i.e., FrontPage). This page includes a mixture of suggested practices, instructions on how to use the wiki, links to other useful pages for beginners, and explanations of how contributors keep the wiki from becoming chaotic and disorganized. In addition to the material about how to use the technology, the following points are raised:

- It is "advisable (but not compulsory) to set your user name on the Preferences page.” The policies recommend that your name conform with a particular format (e.g., DerekHansen).

- Members are told that vandalism (i.e., spam) can easily be removed by "the next well meaning visitor" who fixes them, and that the RecentChanges page (and its RSS feed) can be useful in monitoring recent edits.

- Wikis are "an ideal medium for refactoring.” In other words, members can organize content and make it coherent with a few minutes of cutting and pasting.

There are additional wiki policy and help pages linked to from the bottom of the FrontPage. The following suggestions are scattered throughout these pages:

- Members who want to practice making edits should do so on the SandBox page. 
- Members should choose titles for pages that are descriptive of the content and specific.

- It is "good manners" to sign new contributions with a link to a personal biography page. However, signatures should be avoided when contributions are small (e.g., fixing a typo) or if it doesn't matter if others know who posted the information.

- $\quad$ Pages can use Document Mode (where the content is like an article with commentary expressed at the bottom) or Thread Mode (where signed comments are manually organized into threads).

- Members should not remove or rewrite prior comments entirely. Instead they should "leave the original text as a reference. Much like a conversation, all that is said is valid. All that is added is heard."

In addition, a few additional links are provided to pages found on the WikiWikiWeb website (Cunningham).

Nearly all of the css-d policy pages have remained unchanged or only slightly modified since April 2003 (the earliest time period for which I have data).

\section{Appropriate Wiki Content}

Members are also encouraged to read the ToWikiOrNotToWiki page, which is linked to at the top of the FrontPage. It is a comparison of the types of information most appropriate for the email list and the wiki respectively. The contents of this page as of January 2007 are shown below:

\section{When to Wiki, When to Discuss}

Do you have a question to ask or tip to impart and are not sure where to post it?

\section{To Discuss}

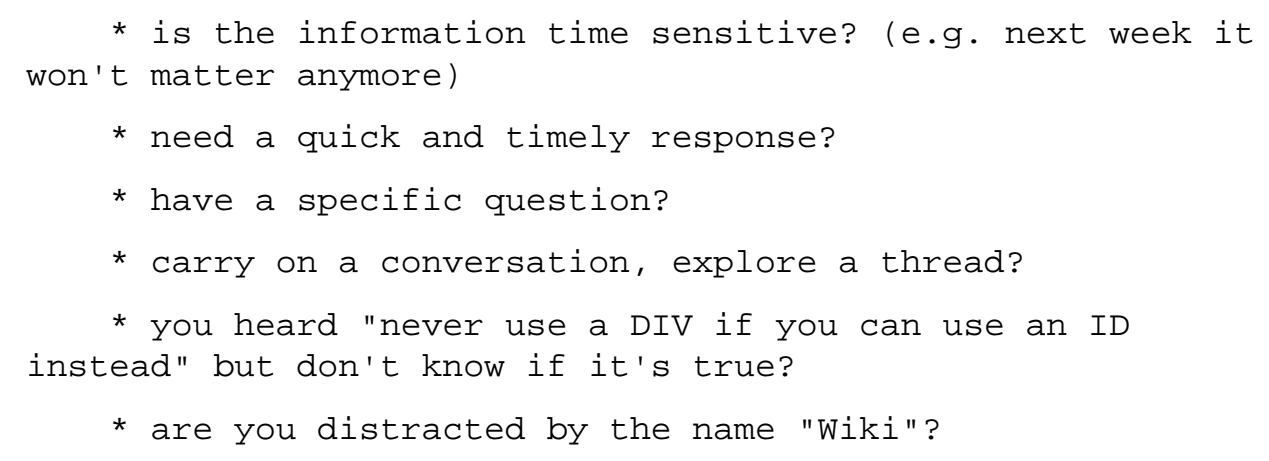




\section{To Wiki}

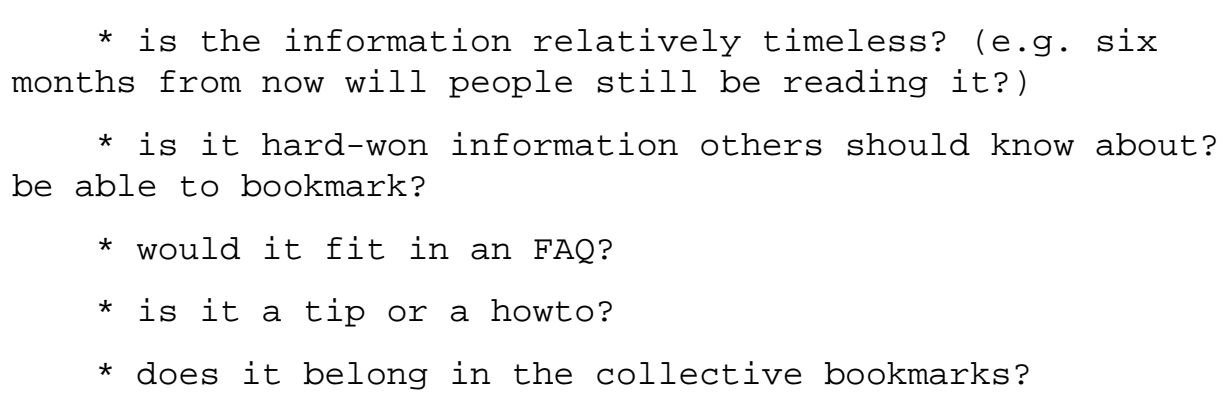

While the email list policies focus on the appropriateness of the subject-matter and whether it is on-topic (e.g., CSS related, practical in nature), the wiki policies focus on the qualities of the information itself (e.g., "time sensitive”) and its intended use (e.g., “would fit in an FAQ”). In fact, several pages on the wiki pertain to website topics that are of interest to the community, but are not considered on-topic in the email list (e.g., Javascript techniques). This was a concern to one member who posted the following comment during 2003 on the WikiSuggestions page, “The number of off topic pages on this wiki seems to be growing. Perhaps there needs to be some effort to reduce them?” A frequent list and wiki participant replied to this comment by creating the OffTopic page, which listed several off-topic content areas for the email list, provided other forums where those topics would be considered on-topic, and mentioned that "you might find limited information about them on this Wiki where they impinge on the practical use of CSS.” The implications of allowing more off-topic content on the wiki than on the list will be discussed in Chapter 6.

For the most part, the content in the wiki matches the suggestions on the ToWikiOrNotToWiki page. There are a few examples of people trying to hold conversations or get answers to questions on the wiki, but they are relatively infrequent. One interviewee (who was not a member of the email list) posted a question to the wiki and the slow response by only one individual made it clear that the wiki was not a good place to ask for immediate help.

\section{Genres and Activities}




\section{Content Overview}

As of May 2006, the entire css-d wiki included over 100,000 words (750 KB of content), spread across 546 pages (see Figure 5.1). (For comparative purposes that is about the size of the text from this dissertation!) The distribution of page size has remained relatively stable over time. Data from May 30, 2006 shows an average page size of $1.4 \mathrm{~KB}$ (e.g., 210 words). The distribution is highly skewed (Gini coefficient of 0.68). The largest page, PrintStylesheets, includes $16 \mathrm{~KB}$ (2.1\% of all KBs), and the largest 54 pages (10\% of all pages) account for half of the total wiki content.

The 546 wiki pages available May 30, 2006 have been categorized into different content areas to give an idea of the overall makeup of the wiki (See Table 5.1). Although there is some overlap with the wiki genres presented in the next section (e.g., Biography Genre), these categories are only intended to convey content areas, not elements of form and purpose.

Table 5.1: css-d Wiki Page Statistics

\begin{tabular}{|l|l|l|l|l|l|l|}
\hline Content & Size & \% Size & Pages & \% Pages & Edits* & \% Edits* \\
\hline Web Design & 599,489 & $78 \%$ & 292 & $53 \%$ & 3,424 & $71 \%$ \\
Biography & 54,777 & $7 \%$ & 144 & $26 \%$ & 545 & $11 \%$ \\
Wiki Admin & 67,799 & $9 \%$ & 36 & $7 \%$ & 487 & $10 \%$ \\
Site Check & 17,782 & $2 \%$ & 22 & $4 \%$ & 123 & $2 \%$ \\
Email List & 16,176 & $2 \%$ & 9 & $2 \%$ & 99 & $2 \%$ \\
Category & 7,990 & $1 \%$ & 12 & $2 \%$ & 83 & $2 \%$ \\
Spam/Junk & 3,710 & $0 \%$ & 31 & $6 \%$ & 84 & $2 \%$ \\
\hline Total & 767,723 & $100 \%$ & 544 & $100 \%$ & 4,845 & $100 \%$ \\
\hline *Edits represent the total number of page edits for all pages within the 'Page Type,' however the pages \\
SandBox and FrontPage with 881 and 656 edits per page were excluded from these numbers, since they \\
serve different purposes that require significantly more editing than the rest of the pages. \\
\hline
\end{tabular}

Just over half of the wiki pages include content related to CSS or website design more generally ('Web Design’ category). These pages are the largest and are frequently edited, accounting for $78 \%$ of the total wiki Bytes and $71 \%$ of all edits. A quarter of the 
pages include personal information about an individual such as contact information, personal websites, and topics of particular interest. Although these 'Biography' pages account for $26 \%$ of all pages, they only account for $7 \%$ of all wiki Bytes and $2 \%$ of all wiki edits. The ‘Wiki Admin' and 'Email List' pages provide background for newcomers learning how to use the wiki or the email list and explain policies. The 'Wiki Admin' pages also include dynamically generated pages (e.g., OrphanedPages), and pages for suggestions to the wiki (e.g., WikiSuggestions).

The 'Site Check’ category includes pages where members ask for feedback on their website (similar in purpose to the Site Check genre of the email list, as discussed in Chapter 4). The 'Category' pages include pages used to organize content through the use of backlinks. Finally, the 'Spam/Junk’ pages include non-webdesign related pages, accidentally created pages, and test pages with no apparent ongoing purpose.

In general, the topics addressed in the wiki closely mirror those discussed in the email list. Content is organized for browsing on the wiki's FrontPage, which contains an annotated list of links to just over 100 specific wiki pages organized into various categories and subcategories. Some of these include "css-discuss mailing list," "CSS Concepts,” “CssLayouts,” “Tips \& Tricks,” “Testing,” “Learning,” “Debatable,” “Sizing Text," and "Meta" (pages that deal with the wiki itself). Although these content areas are similar to those discussed on the list, the form the information takes and the flow of communication is very different as outlined in the following sections.

Unfortunately, I could not analyze the wiki server log reports to determine which pages are most frequently accessed. However, I could get an idea of the most frequently accessed pages via search engines by analyzing search phrases and the pages they ended up at. The 100 most frequently used search phrases from June 2004 and January 2007 were analyzed. These accounted for 38\% (May 2004) and 30\% (January 2007) of all search phrases during each month. I entered each search phrase into Google (on July 21, 2007) and associated the first wiki page listed in the search results with each wiki phrase. 
Using Google was appropriate as over 91\% of the searches occurred on Google. Ideally, the searches would have been performed during the original time period, as search results vary over time. However, the fact that a wiki page was found in the search results in all but 3 cases suggests that the approach worked reasonably well, although it is possible that some of the pages suggested recently are different than the ones originally suggested by Google.

The analysis suggests that most individuals coming to the wiki from search engines end up at specific wiki pages rather than the wiki Front_Page. All but two of the pages arrived at via search engines were in the 'Web Design' category (the others were classified as 'Email List' and 'Spam'). The analysis also suggests that some pages are accessed via search engines far more often than others. Table 5.2 shows the wiki pages most often accessed via search engines based on the analysis for the two months. It is noteworthy that many of the same pages were among both lists, even though the searches were performed several years apart.

Table 5.2: css-d Wiki Pages Most Often Accessed via Search Engines

\begin{tabular}{|l|l|l|l|l|}
\cline { 2 - 5 } \multicolumn{2}{l|}{} & \multicolumn{2}{l|}{ May-04 } & Jan-07 \\
\hline Page Name & rank & searches & rank & searches \\
\hline ThreeColumnLayouts & 1 & 408 & 1 & 4,987 \\
\hline BoxModelHack & 2 & 365 & 7 & 921 \\
\hline Front_Page & 3 & 360 & 9 & 816 \\
\hline StyleInEmail & 4 & 218 & 16 & 304 \\
\hline FontSize & 5 & 186 & 3 & 1,743 \\
\hline CssLayouts & 6 & 174 & 6 & 1,119 \\
\hline RoundedCorners & 7 & 149 & 5 & 1,307 \\
\hline CssHack & 8 & 132 & 2 & 3,981 \\
\hline VerticalAlign & 9 & 123 & 15 & 332 \\
\hline ImportHack & 10 & 98 & 4 & 1,691 \\
\hline CssEditors & 21 & 18 & 8 & 875 \\
\hline FormElements & N/A & N/A & 10 & 690 \\
\hline
\end{tabular}




\section{Wiki Genres Repertoire}

Activity on the wiki is typically organized around individual wiki pages. Pages are created, added to, and refined, through an iterative process. Wiki pages typically stand on their own, although they may link to other related pages. As the wiki policy suggests, the title of a page is important in signaling what the content on the page is about. Pages on the css-d wiki are primarily organized through the FrontPage, which serves as an index, and hyperlinks that connect related pages. Most content is descriptive and prescriptive in nature. Rather than being a question and answer format, most information is presented as facts, methods on how to accomplish something, and opinions that are typically signed by the author. Many pages are edited years after they were initially created, in contrast to email threads that typically only last a few weeks at most.

The genre repertoire for the wiki includes several types of wiki pages as outlined in Table 5.3. Some of these are referred to by the community including Biography pages (also called Home Pages by the community), Site Check pages, and Debate pages, while the others are not. The newly proposed genres are not entirely foreign to how members describe the wiki contents, they are just more generalized. For example, members talk about the "layout pages" as having similar aspects of form and purpose (i.e., including lots of links to page layouts and serving the purpose of finding an appropriate layout for one’s specific needs). Most of these pages are a subset of the more general Annotated Links genre that I propose, which includes the layout pages, as well as pages such as CssEditors and GoodBooks. These other pages are similar to the layout pages in form and purpose, but they are not specific to the content area of page layouts. I have taken this analytic approach in order to explore issues of more general appeal than CSS content. As is typical of genre studies, not all pages fit into a definite wiki genre and some fit into multiple genres. This is especially true on the wiki because genres occurring in new media take time to become standardized (Yates \& Orlikowski 1992). 
Table 5.3: css-d Wiki Page Genres

\begin{tabular}{|c|c|c|c|c|c|}
\hline $\begin{array}{l}\text { Wiki } \\
\text { Genre }\end{array}$ & Purpose & Authors & $\begin{array}{l}\text { Contribution } \\
\text { Size }\end{array}$ & Form & Examples \\
\hline $\begin{array}{l}\text { Annotated } \\
\text { Links }\end{array}$ & $\begin{array}{l}\text { Help user find } \\
\text { appropriate, } \\
\text { existing resource }\end{array}$ & Many & $\begin{array}{l}\text { Small (single } \\
\text { entry) }\end{array}$ & $\begin{array}{l}\text { List of } \\
\text { references } \\
\text { with } \\
\text { annotations }\end{array}$ & $\begin{array}{l}\text { ThreeColumnLayouts, } \\
\text { CssEditors, CssHack, } \\
\text { DropShadows, } \\
\text { GoodBooks }\end{array}$ \\
\hline 'How To' & $\begin{array}{l}\text { Explain how to } \\
\text { accomplish } \\
\text { something }\end{array}$ & $\begin{array}{l}\text { Few or } \\
\text { Many }\end{array}$ & $\begin{array}{l}\text { Medium to } \\
\text { Small }\end{array}$ & $\begin{array}{l}\text { Procedural } \\
\text { knowledge; } \\
\text { organized by } \\
\text { competing } \\
\text { approaches } \\
\end{array}$ & $\begin{array}{l}\text { BoxModelHack, } \\
\text { ConstantsInCss, } \\
\text { ClearingSpace, } \\
\text { CenteringBlockElement, } \\
\text { StyleSwitching }\end{array}$ \\
\hline Biography & $\begin{array}{l}\text { Introduce oneself } \\
\text { to community }\end{array}$ & Single & $\begin{array}{l}\text { Small to } \\
\text { Medium }\end{array}$ & $\begin{array}{l}\text { Description } \\
\text { of self }\end{array}$ & $\begin{array}{l}\text { Not included for privacy } \\
\text { reasons }\end{array}$ \\
\hline Debate & $\begin{array}{l}\text { Outline } \\
\text { competing } \\
\text { arguments and } \\
\text { reduce } \\
\text { contentious and } \\
\text { off-topic } \\
\text { conversation on } \\
\text { list }\end{array}$ & Few & Medium & $\begin{array}{l}\text { Self- } \\
\text { identification } \\
\text { of } \\
\text { confrontation } \\
\text { al nature; pro } \\
\text { and con } \\
\text { discussion or } \\
\text { list }\end{array}$ & $\begin{array}{l}\text { TablesVsDivs, } \\
\text { TablesVsLists, } \\
\text { UsingFontSize, } \\
\text { ToHackOrNotToHack }\end{array}$ \\
\hline Article & $\begin{array}{l}\text { Provide an } \\
\text { overview of a } \\
\text { topic, describe } \\
\text { how something } \\
\text { works, and/or } \\
\text { present results } \\
\text { from tests or } \\
\text { surveys. }\end{array}$ & $\begin{array}{l}\text { Few to } \\
\text { Many } \\
\text { (often } \\
\text { single } \\
\text { editor) }\end{array}$ & $\begin{array}{l}\text { Medium to } \\
\text { Large }\end{array}$ & $\begin{array}{l}\text { Primarily } \\
\text { declarative } \\
\text { knowledge; } \\
\text { long; links to } \\
\text { further info }\end{array}$ & $\begin{array}{l}\text { PrintStylesheets, } \\
\text { OverlappingAndZIndex, } \\
\text { CodeValidation, } \\
\text { ProgressiveEnhancement }\end{array}$ \\
\hline Definition & $\begin{array}{l}\text { Defines a term or } \\
\text { phrase. }\end{array}$ & $\begin{array}{l}\text { Single } \\
\text { author or } \\
\text { few }\end{array}$ & $\begin{array}{l}\text { Small to } \\
\text { Medium }\end{array}$ & $\begin{array}{l}\text { Declarative } \\
\text { knowledge; } \\
\text { brief }\end{array}$ & $\begin{array}{l}\text { DocumentTypeDefinition, } \\
\text { QuirksMode, } \\
\text { ChildSelector, DocType }\end{array}$ \\
\hline
\end{tabular}

\section{Annotated Links Genre}

One particularly successful genre on the css-d wiki is what I call the Annotated Links genre. An example, the ThreeColumnLayouts pages is shown in Figure 5.7. While this is not a name used by the community, it captures the key characteristics of a set of pages that are similar in purpose and form. Some of the prototypical example pages from this genre include: ThreeColumnLayouts, CssEditors, CssHack, GoodBooks, DropShadows, and ShowCase. The purpose of these pages is to help an individual 
compare and select an appropriate resource (e.g., layout, book, text editor, wiki page).

The bulk of the content is a list of links with brief descriptions of each.

Figure 5.7: ThreeColumnLayouts Page (Excerpts)

\title{
ThreeColumnLayouts
}

FrontPage | RecentChanges | Preferences

[Home]

\begin{abstract}
The question of finding good 3 column layouts comes up in the mail list from time to time. Here are some that I have found, along with a few notations about what features they include and how they work. The judgment of "good" is left to you and your needs. Each offers interesting techniques for the CSS student. By the way, when visiting these places, look around; many of these people offer other layouts that I have not included here.
\end{abstract}

I'm sure there are more that I have missed. Add the others you know, or mail me and I'll add them. of course, don't hesitate to mail me about any errors or misrepresentations I've made.

- Enjoy!-

\begin{tabular}{|l|l|l||l||l|l||}
\hline layout & header & footer & fluid & NN4 & notes \\
\hline [Position is Everything's] One True Layout & Y & Y & Y & Y & $9,34,35$ \\
\hline [rayRO's] 3col bg, validating XHTML\&CSS (no hacks!), 2-1-3 ordering & Y & Y & N/Y & N/Y & 9 \\
\hline [rayRO's] 3col background-color Layout & Y & Y & Y & N & 22, 32, 33 \\
\hline \hline [Rob Chandanais'] Blue Robot "3 col Flanking" & N & p & Y & N & $1,7,29$ \\
\hline [Eric Costello's] glish.com "3 columns, the holy grail" & Y & N & N & N & 1 \\
\hline \hline [Holly 'n John] "Three Column Stretch" & Y & Y & Y & N & 1 \\
\hline \hline [Mark Newhouse's] nn4_3col_header & Y & Y & Y & Y & $1,2,3$ \\
\hline
\end{tabular}

\section{[Page Cropped]}

Notes about technique:

1. Central content column has large margins. Absolute positioned menu/sidebar columns sit in the left and right margins of the central column.

2. Rightmost column is positioned absolute using a \% distance from the LEFT so that it works in NN4.

3. Needs, and includes, a script to reload the page when re-sizing Netscape 4.

4. Each column is absolutely positioned and uses $\%$ widths.

5. Illustrates the use of IE Conditional Comments to accommodate IE5. Explore!

6. Uses HRs to force minimum widths in some browsers.

7. Footer spans only the central content column.

8. Absolute positioned central content column has a large left margin. The absolute positioned left columns sits in the left margin of the central column. The right column is defined as in note \#2.

q. All floats.

As is typical of this genre, the page begins with a paragraph describing what the page is about and encouragement to add other links. This is not always in the first person, but is in this case. Other pages include information about the selection criteria that should be used when adding links. For example, the ShowCase page, a list of good CSS designs, includes the following recommendation: "Any one individual should add no more than two of their favorite sites, and should not add their own work: if your site is good enough, someone else will notice and add it.”

The ThreeColumnLayouts page includes links listed in a table format with comments about the links shown in the table fields to the right of the link and notes at the 
bottom. The tabular format allows for easy comparison of various layouts on a few dimensions, although the notes section makes it clear that not all useful information about the layouts fits into the structured form. The content of the annotations relates to the specific techniques used to achieve the layout (e.g., absolute positioning), special features of the layout (i.e., includes a footer), and browser support for the layout (e.g., NN4 support). These are all important factors for a designer to consider when selecting a specific layout to use as the base for her website.

More often, links are displayed in bulleted lists (e.g., ShowCase, GoodBooks, CssEditors). These lists are often organized into sections using headers and include specific comments to the right of each link. Although the text of the CssEditors page is not readable in Figure 5.8, the structure of the page is evident.

\section{Figure 5.8: CssEditors (Excerpt)}

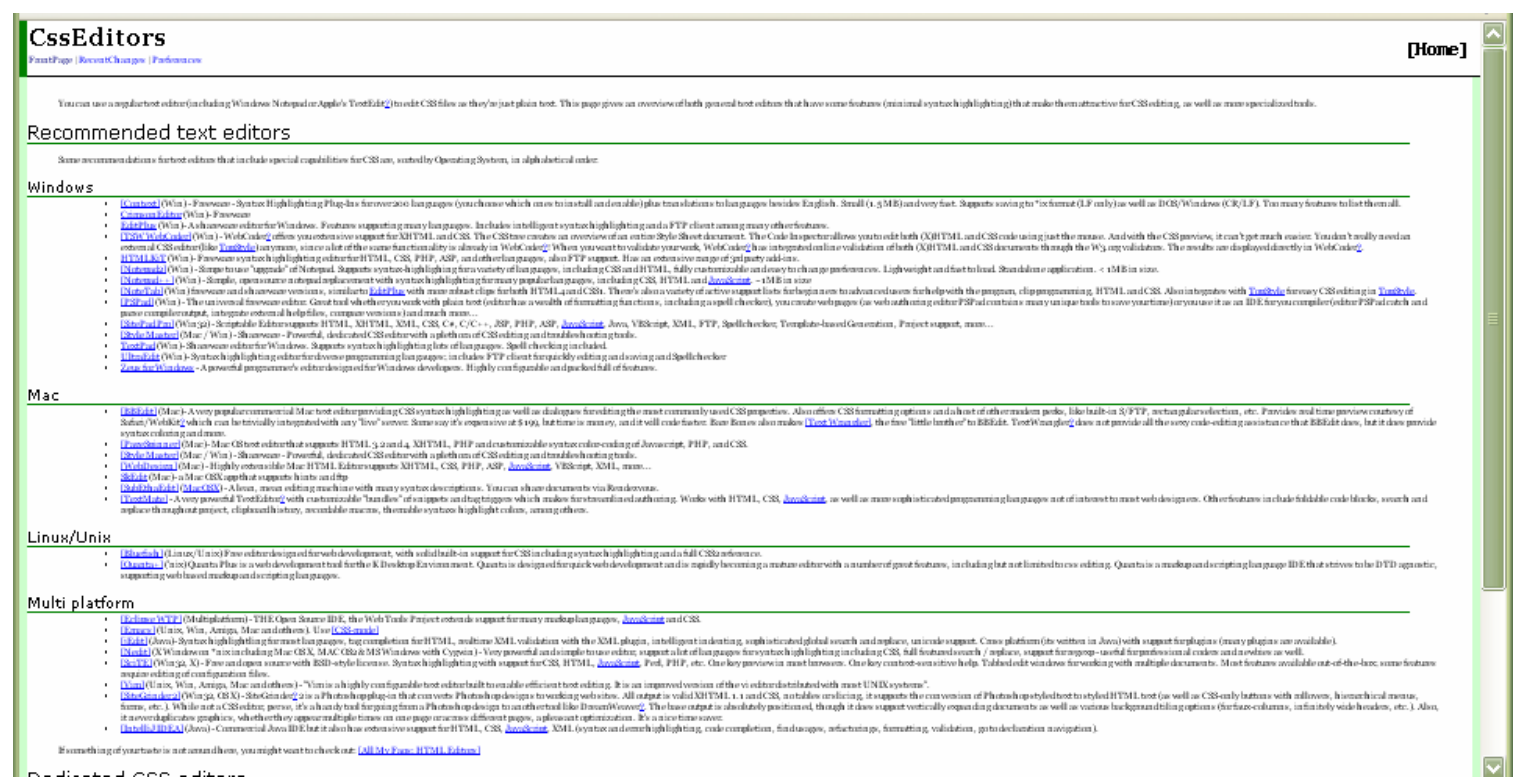

Annotated Links pages are typically among the most frequently edited pages. The structured nature of the content makes it clear to visitors what a contribution should look like (i.e., an additional link with a description). Because a typical contribution is small, little effort is required to make one. However, the aggregate value of them together can be great. This is especially true in situations where the knowledge about the items is 
distributed among many individuals. For example, the ThreeColumnLayouts page includes links to many design layouts that are scattered all over the web on individual designers' websites. Members agree that it is hard to know of all of them and that the ThreeColumnLayouts page is a one-of-a-kind resource because of its ability to pull them all together in one place. This is supported by the fact that it is the most popularly referenced wiki page in the email discussion (see Table 6.2), and the most popular wiki page referred to by search engines in the two months analyzed (See Table 5.2). Other Annotated Links pages such as CssEditors and CssHack are also commonly referred to by search engines (Table 5.2) and list members (Table 6.2).

\section{‘How To’ Genre}

Because the css-d community is interested in the "practical use of CSS," much of the content in the email list and wiki is procedural in nature. Although people do describe "why" some things happen, they are mostly interested in "how" to accomplish various things using CSS. As a result, many wiki pages are primarily 'how to’s. Because of the nature of CSS, these 'how to' pages often include one goal (i.e., create rounded corners on a website layout) with many different techniques. Each technique has its own drawbacks (e.g., it’s not supported by a certain browser) and situations where it is not appropriate to use (e.g., won’t work with a fixed position layout). Many of the wiki pages capture this type of knowledge as well. Thus, the purpose of a 'How To' page at css-d is not simply to show a single solution. Rather it is to display several potential solutions along with information that will help people decide which one is best for their particular situation. A few pages typical of this genre are BoxModelHack, ConstantsInCss, ClearingSpace, CenteringBlockElement, StyleSwitching, and MysteryBug. 
The ConstantsInCss page discusses several methods for updating colors in a CSS file, since CSS does not provide a way to declare a constant. After discussing the problem, several “options” are listed as quoted below:

\section{Option 1: Search and Replace}

The simplest option is just to include the color value in every rule you want it in, and use search and replace when you want to change it. This is pretty trivial with a good text editor.

\section{Option 2: Create a Class for the Constant/Variable}

Since an element can have more than one class on it (see Multipleclasses), you can make classes for each constant or variable you want to create, then assign these classes to the (X)HTML elements you want to affect. Since an element can have more than one class on it, this shouldn't interfere with any existing uses of the class attribute.

for example: [CSS code included below]

Then if you want to change the color, all you need to do is change the class definition.

\section{Disadvantages}

* If used extensively, the (X)HTML can get pretty bulky with all those extra classes assigned.

* The author has to remember to assign the correct class. This can be especially problematic if the content creator is not the same person who created the css (an unknowing client, for instance, who is maintaining her own page) and has to be told when and where to add a class.

* Not all browsers support this.

Option 3: Use a Server-Side Language to Write out the cSS [discussion of technique with PHP code example and ideas for other scripting solutions]

\section{Examples}

* PHP script by John Daniels [hyperlinks to outside resources]

* PHP script by Tom Hall

* Article by Seth Richards

\section{Disadvantages}

* CSS is not cached (but this can be worked around?).

* The cSs author needs to know the programming language. 
The organization of the page into various competing techniques is typical of this genre. So is the inclusion of actual code, a vital part in showing how to accomplish something. Although some of the page discusses the practical procedural knowledge needed to accomplish the various methods, an equal amount of content compares the various methods. This is also typical of this genre. This can be done in bulleted form as in this example, or through signed comments at the end of various methods. For example, the following signed comment is shown on the ClearingSpace page at the end of "Option 5” (i.e., the 5th method for clearing unwanted space discussed on the page).

Something to consider regarding this option: when a page
using this method is viewed in a css-incapable Useragent,
the horizontal rule would act as an "end-of-DIV" marker
that may or may not be desired. Options 2 and 3 shouldn't
be nearly as obvious. -- JosephTanner

The CssInConstants Option 3 example regarding server-side scripting languages points out that not all content in these How To pages needs to be strictly CSS-related. Unlike the email list where discussions of scripting languages and inclusion of PHP code are discouraged, there is no evidence that members are discouraged from including this information on the wiki. In fact, this particular content was added by an administrator on the email list.

Several of these 'How To' pages are commonly referred to from the email list or search engines. For example, the BoxModelHack and Rounded Corners pages are among the most commonly referred to from both sources (see Table 5.2 and 6.2).

\section{Biography Genre}

The socially recognized purpose of the Biography or Home Page genre is to introduce oneself to the community and make it possible for your username to show up on the RecentChanges page when you make edits. These pages are typically separate from other genres and fairly easy to identify. They comprise 144 (26\%) of the 544 wiki 
pages, although they are relatively small pages so they only account for only $7 \%$ of all wiki content (as measured in bytes) (see Table 5.1). Because of the personal nature of these messages, names have been changed and content from different Biography pages has been combined in the examples that follow.

Biography pages typically include the person's username as the title. This may be in the form of a WikiWord (e.g., "JohnSmith,” "GreyWolf”) as suggested in the wiki policy pages or may be a single word (e.g., "steve,” “James”). Biography pages contain an average of approximately 50 words per page. Some of them contain only a name, suggesting that they were created simply to show the username appropriately on the RecentChanges page. Most of them include a few words about the person and link to a personal blog or website they are developing. The following is typical:

I am a 26 year old Computer Science graduate from Austin TX, currently working for abc.com

I have an occasionally updated blog: [URL]

Specific contact information such as phone numbers and mailing addresses are rarely provided, but email addresses are occasionally shared. People often share specific web design topics they are interested in (e.g., "I'm interested in webstandards, CSS and XML” or “Besides CSS, I'm interested in the Opera browser and PHP programming”). Rarely, individuals mention their role within the css-d community as in the following examples taken from different Biography pages:

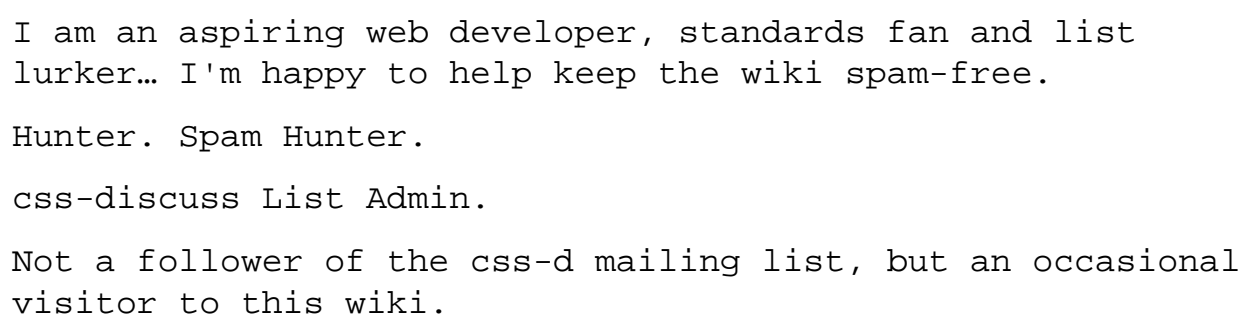

A few active wiki participants use their own Biography page as a place to share their personal opinions and accomplishments on website design issues. For example one Biography page included several paragraphs outlining the author's position on the 
inappropriateness of using CSS hacks. Others included "rants" or "ramblings" about CSS topics and their personal position related to them (e.g., "Most of the page's text size should be set to medium. It’s the default font size for a reason!”). Other Biography pages list “css-accomplishments” and various technical skills (e.g., “SQL, XML, C++”).

Finally, a few Biography pages are used as a place to have a discussion with the owner of the page. This practice is also common on other wikis such as Wikipedia. When authors leave a messages on another member's Biography page they typically sign the comment with a link to their own Biography page. For example, one member left the comment “Awesome job! Thanks a lot :)” followed by a signature on the Biography page of a self-declared "spam hunter.” In a few other cases individuals have a brief threaded conversation on a Biography page. For example, three members debated the appropriateness of removing a particular link on a website from within a member's Biography page. These discussions typically occur among active wiki contributors who have had experience with other wikis and are not active list participants. In this way the Biography pages serve as a backchannel for personal communication among wiki users.

Biography pages are not used in the email list discussion or frequently accessed via search engines. This suggests that their use is limited to coordinating work and introducing oneself to other wiki contributors. This is particularly important for individuals who are not part of the email list, where members get to know one another through frequent interaction.

\section{Debate Genre}

The purpose of the debate genre is not necessarily to carry out a debate, rather it is to outline competing arguments in a well-known debate (i.e., Holy War). This genre is relatively rare, but its unique purpose and relationship with the email list make it worth exploring in some detail. The debate genre was originated by administrators who wanted to outline the arguments of various Holy Wars on the wiki so that members would not 
have to revisit them in the email list discussion. This explains the introductory statement: “Since HolyWars are by their nature long winded and boring let’s keep this one tucked away on its own page where it won’t bother anyone who doesn’t want to be bothered.” Some prototypical debate pages include: TablesVsDivs, TablesVsLists, FontSize (and other related pages such as UsingFontSize), and ToHackOrNotToHack. Many of these, and a few other pages that include individual's opinions on contentious topics are grouped together under the heading "Debatable” on the wiki's FrontPage. Portions of the ToHackOrNotToHack page are shown in Figure 5.9.

Figure 5.9: ToHackOrNotToHack (Excerpts)

\section{ToHackOrNotToHack}

FrontPage | RecentChanges | Preferences

[Home]

The topic of whether or not to use CssHacks is often hotly debated, and could be thought of as a HolyWar. Since HolyWars are by their nature long winded and boring let's keep this one tucked away on its own page where it won't bother anyone who doesn't want to be bothered.

\section{Arguments For CSS Hacks}

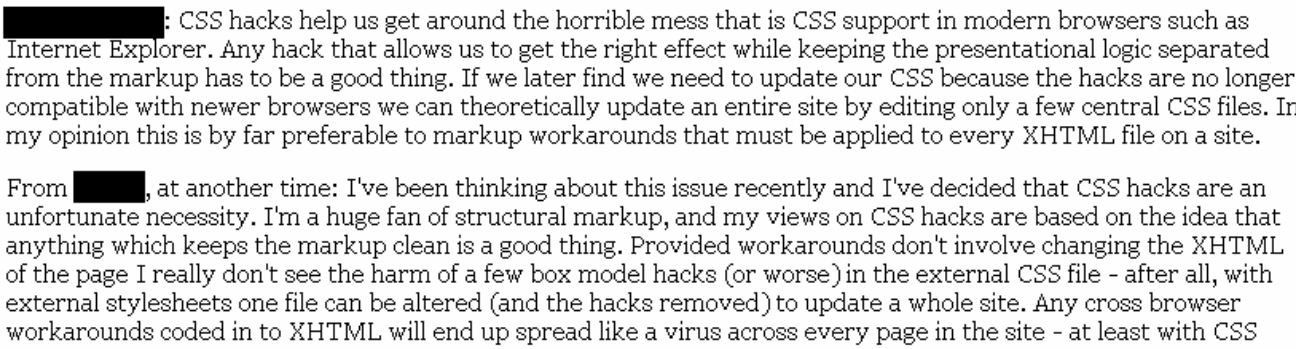

[Page Cropped]

\section{Arguments Against CSS Hacks}

has written his views on his personal page.

Interesting thoughts: http://allmyfaqs.com/faq.pl?Fix_the_wrong_problem

Why CSS hacks are evil: http://www.digital-web.com/columns/keepitsimple/keepitsimple_2003-11.shtml

PPK thoughts on CSS Hacks: http://www.quirksmode.org/css/csshacks.html

\section{The origins of the box model hack and a reflexion on} hacks

Tantek Celik, creator of the box model hack and many famous others, wrote on September 262005 about its genesis and consequences: "Pandora's Box (Model) of CSS Hacks And Other Good Intentions" (http://tantek.com/log/2005/11.html\#d26t1820) is an essential read about CSS hacks. 
As with other debate pages, the topic of this page is openly recognized as a "hotly debated” topic and a Holy War. In this case the debate is about the appropriateness of using CSS hacks, or CSS workarounds used to target different browsers. Other pages like the FontSize page outline the debate in a neutral tone and discuss why it is contentious.

The following excerpt is telling:

Some authors argue... The flip side of the debate holds... Both approaches have their strengths and weaknesses, and it is left to the individual to decide which he or she would prefer to use. To assist in making that decision, here are some articles, examples, opinions, and discussions from both sides of the fence.

The structure of a Debate page is organized around the arguments "for" and "against” the different positions. Figure 5.9 shows the use of headers to clearly delineate these sections. Other pages, such as the UsingFontSize page use tables to compare various alternatives as shown in Figure 5.10 below:

Figure 5.10: UsingFontSize (Excerpt)

\section{UsingFontSize}

FrontPage | RecentChanges | Preferences

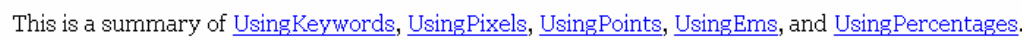

Also see: $\underline{\text { AvoidingHacks }}$

\section{Summary}

\begin{tabular}{|c|c|c|}
\hline Method & Advantages & Disadvantages \\
\hline Keywords & $\begin{array}{l}\text { Not influenced by nearby font sizes so easier to } \\
\text { manage }\end{array}$ & Limited choices, only 7 sizes \\
\hline Pixels & Consistent across browsers & $\begin{array}{l}\text { Fixed size disregards user needs. Physical size is smaller } \\
\text { as display resolution is increased. IE/Win buggy, see } \\
\text { ForceFontSize. }\end{array}$ \\
\hline Points & $\begin{array}{l}\text { Good for print style sheets (but with possible user } \\
\text { accessibility problems) }\end{array}$ & $\begin{array}{l}\text { Inconsistent (e.g. between PC and Mac) on screen; } \\
\text { depends upon system DPI, which is rarely set close to } \\
\text { reality on a PC. }\end{array}$ \\
\hline Ems & Relative sizing & Cascade bugs in IE (fixes available) \\
\hline Percents & Relative Sizing & $\begin{array}{l}\text { Cascade math requires a little more thought then } \\
\text { non-relative sizes }\end{array}$ \\
\hline $\begin{array}{l}\text { Ems w/ } \\
\text { Percent Base }\end{array}$ & $\begin{array}{l}\text { Relative sizing / Good browser suport / Centralised } \\
\text { control in the CSS file, Easily overridden by } \\
\text { UserStylesheets }\end{array}$ & Easily overridden by user stylesheet \\
\hline
\end{tabular}

The table works well in this situation because there are many different font sizing techniques that are part of the debate, rather than just two. 
Although Debate pages often include signed comments with people's opinions (e.g., see Figure 5.9 noting that the signed names have been blacked out), they typically do not include personal attacks or a significant amount of back-and-forth discussion. This is a result of the social perception of the css- $\mathrm{d}$ wiki, not the technology itself as is evidenced by the many arguments and flaming that occur on other wikis such as the WikiWikiWeb. Although some members do use a conversational tone at css-d, most members think of it as a repository of information, not a place to debate things. This perception was reinforced by the administrators who initially set up some of the Debate pages using a neutral tone. When talking about creating the FontSize page he mentioned presenting the content "with as little heat as possible on the wiki."

Although Debate pages are few in number, some of them are used frequently in the email list discussion and are referred to by search engines. The FontSize page is the most notable example (see Table 5.2 and 6.2).

\section{Article Genre}

A few pages include a mixture of declarative and procedural knowledge on a broad CSS-related topic (e.g., creating printer-friendly websites; validating CSS code). They are similar in spirit to articles on a given topic, although they are typically not as polished (e.g., they may include bulleted lists instead of polished prose). Article pages are often long and frequently edited. For example, the PrintStylesheets page is over 2,500 words and has been edited 59 times (see Table 6.2). Several of these pages have a selfdeclared editor who originally created the page and maintains it over time. However, others contribute to these pages as well through signed comments and other edits. Some typical pages in this category include: PrintStylesheets, OverlappingAndZIndex, CodeValidation, and ProgressiveEnhancement.

The following quotes are taken from the introduction of the PrintStylesheets page: 


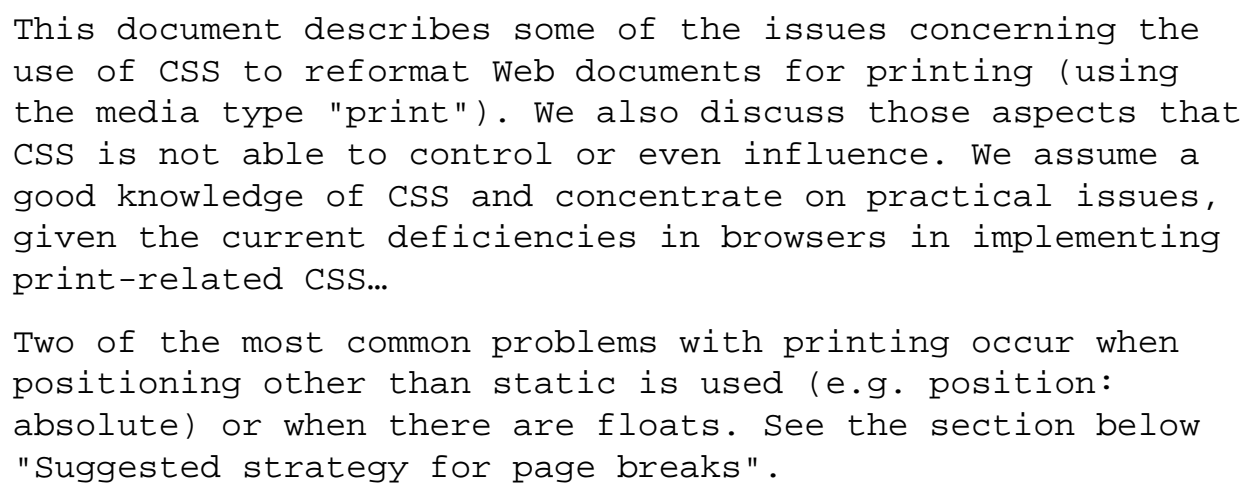

Notice that this page is described as a “document” and is written in a tone more formal than many pages on the wiki. It also introduces the organization of the page and what will be discussed. Rather than quickly jumping to bulleted points as many wiki pages do, the page continues in prose. It covers many subtopics related to creating printer-friendly CSS. These include common problems and solutions to them (e.g., dealing with page breaks), as well as more advanced topics (e.g., high quality printing). Finally, it ends with references to other article on the topic and the following comment: “This page was created by Billy Meyers in June 2003, who welcomes more research; questions; comments; and corrections.”

Some of these pages are commonly referred to by email list members and search engines. For example, the PrintStylesheets and ScreenreaderVisibility are among the most commonly referenced wiki pages in the email list (see Figure 6.2) and are referred to many times by search engines (e.g., they were ranked $13^{\text {th }}$ and $19^{\text {th }}$ most frequently linked to pages in our analysis for May 2004).

\section{Definition Genre}

The Definition Genre includes brief write-ups on a specific CSS concept. These are similar to traditional dictionary entries, except that they also may include examples of the concept in use and comments about its usage (e.g., what browsers don't support it). Many of these pages are linked to from the CssGlossary index page and the CategoryCSSConcept category page. Other pages also link to them when using the term 
that is being defined. Some examples include DocumentTypeDefinition, QuirksMode, ChildSelector, and DocType. These pages rarely include signed comments and appear as if they were authored by a single person. They are not frequently edited, likely because the content in them is relatively stable over time. Although longer than some, the DocType page below is illustrative of several common elements of this genre:

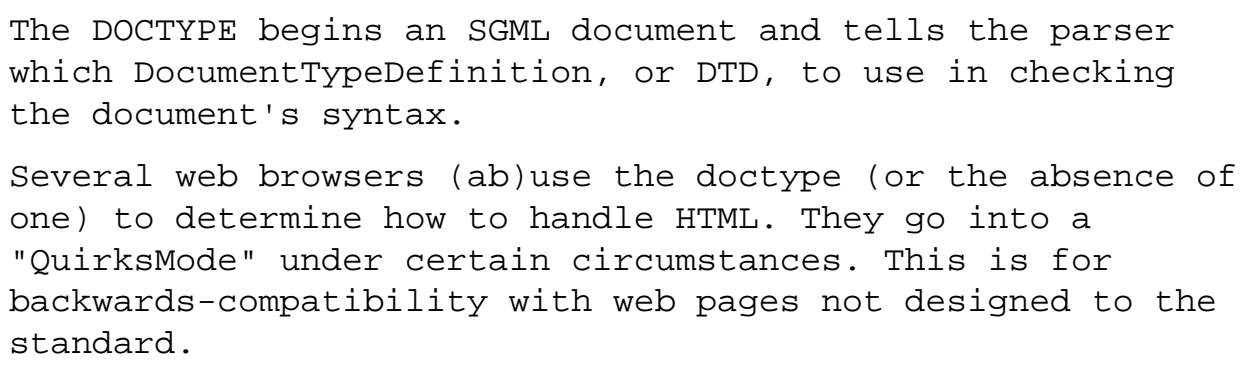

HTML 4.01

\section{Strict}

<!DOCTYPE HTML PUBLIC "-//W3C//DTD HTML 4.01//EN"

"http://WWW.w3.org/TR/html4/strict.dtd">

\section{Transitional}

<!DOCTYPE HTML PUBLIC "-//W3C//DTD HTML 4.01

Transitional//EN"

"http://WwW.w3.org/TR/html4/loose.dtd">

[Five additional examples are shown]

\section{See Also}

[Four external URLs to articles discussing doctypes are included]

As is typical, the DocType page is named after the item it describes (i.e., document types). It begins by describing the item. As is typical, comments about its importance to website designers are included (e.g., "several web browsers (ab)use the doctype...”). As in this case, many of these comments relate to problems that can arise because of the topic. Finally, the page ends with a list of examples and links to related external articles. 
The ShorthandProperty example below demonstrates that these elements are often found even in much smaller Definition genre pages:

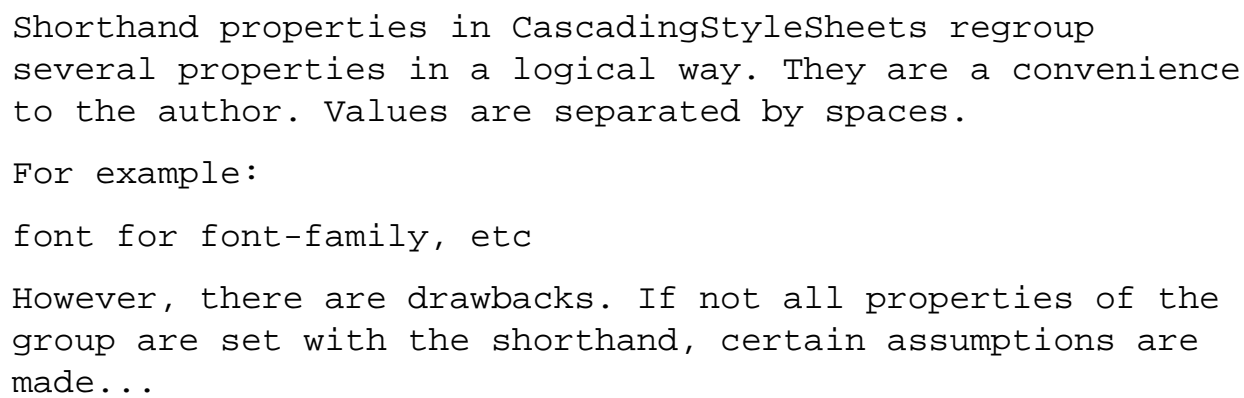

The Definition genre pages are not referred to often in the email list discussion or search engine results. Instead, they are linked to often from other wiki pages as described above.

\section{Failed and Missing Genres}

Before leaving the genre repertoire discussion, I will discuss one failed genre, the Site Check Genre, and one missing genre, the Q\&A genre. These are discussed because they are so central to the work occurring on the email list, yet have not played a significant role on the wiki.

\section{Site Check Genre}

When the wiki was initially set up, one of the individuals who seeded content on the wiki thought it would be a good idea to use the wiki to help with Site Checks (see Chapter 4 for a description of the Site Check genre system in the email list). He created the SiteCheckPlease page which recommended a series of steps that people should take when desiring feedback on their site. Sections of the page are included below:

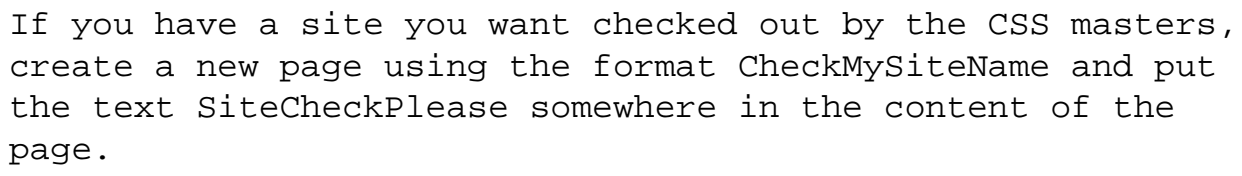




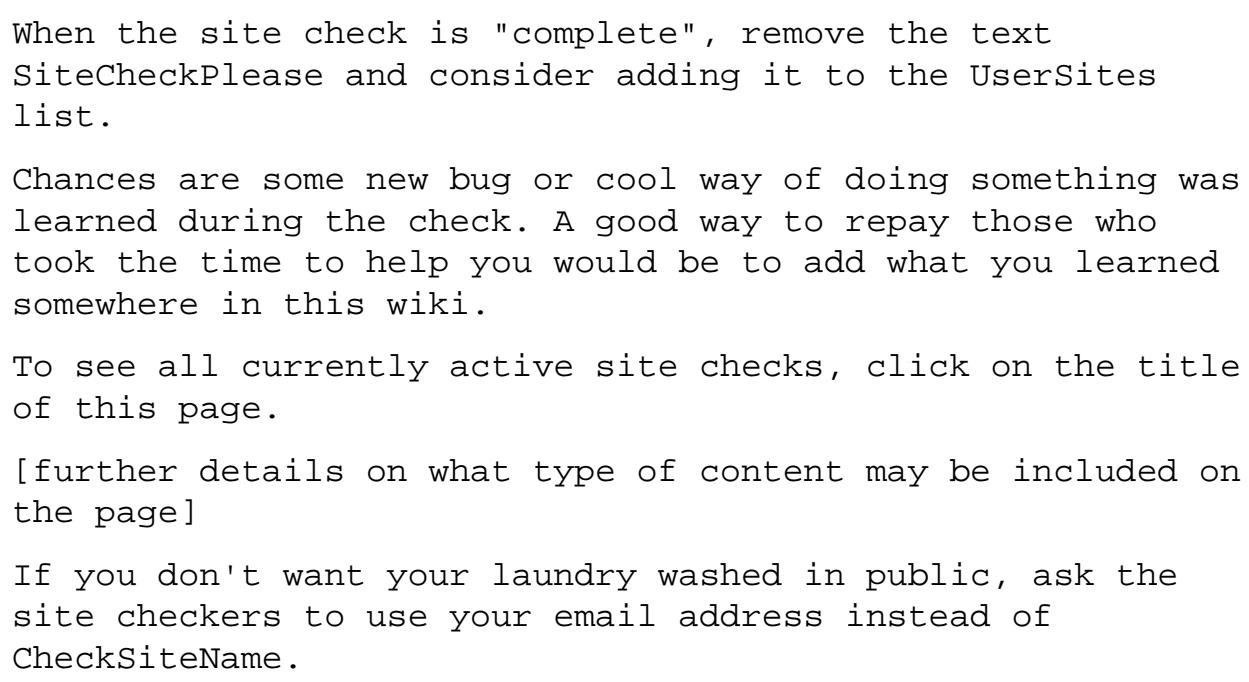

In total, 22 pages following the prescribed format (e.g., CheckAbcCom) were added to the wiki. These wiki pages include the same characteristics as the Site Check genre email messages described in Chapter 4. Of these, 15 (68\%) didn’t receive any feedback on the website, and the most comments anyone received was 3 . In a few cases members were frustrated and subsequently posted messages to the css-d email list asking for a Site Check. List members discussed the appropriateness of the wiki for Site Checks a couple of times, but did not come to a definitive conclusion for several years. Some people argued that having them on the wiki reduced unnecessary traffic on the list, while others argued that the extra step of going to the wiki was unnecessarily burdensome. The confusion of how to use the wiki also contributed to its not being used frequently.

Finally, in late 2006 an email list administrator changed the content of the SiteCheckPlease wiki page so that it sent people to the email list for Site Checks instead of the wiki.

\section{Q\&A Genre}

Noticeably absent from the list of wiki genres is a Q\&A genre, which serves as the foundational genre of a traditional FAQ document. There are relatively few actual question and answer pairs on the wiki, despite the fact that the majority of content was 
created in order to help answer questions in the email list. One person attempted to create an FAQ page on the wiki, but nobody added to it and nobody has referred to it on the list. Interviewees suggested that this was the case because the Q\&A format was too restrictive and people thought of the entire wiki as an FAQ document, so creating a single FAQ page seemed pointless. To be clear, questions (especially frequently asked questions) still drive the majority of the wiki content; it is the presentation and emphasis of that content that is significantly different than a traditional FAQ.

Interviews suggested that this is due to the complexity of the question and answer landscape and the importance of context in satisfying an individual's information need. For example, most questions take the form "how do I perform X?" where there are several ways of performing $X$, each with their own set of contingencies, costs, and benefits. For example, one popular question is: "how do I create a three column layout using CSS instead of tables?” As is typical, there is more than one solution. As we saw, dozens of them are catalogued on the ThreeColumnLayouts wiki page (see Figure 5.7), each with a list of characteristics and notes on techniques used to achieve them. It quickly becomes apparent that a single question and answer pairing would be insufficient, as there are too many answers and issues to think about when selecting one. The opposite case also occurs, where there are many situations that require the use of a particular technique (e.g., a browser hack).

\section{Wiki Content Problems}

Although many individuals have praised the wiki for its valuable content, it is not without its problems. Interviews and content analysis revealed two major problems with the wiki: its inclusion of outdated information and its lack of consistency.

Many pages have remained unaltered for years, even though the CSS specifications and browsers have changed considerably, making the original suggestions outdated. Content is rarely deleted from the wiki. In at least one case when a member 
deleted what they considered to be an outdated best practice, another member put it back in explaining that they still thought it was good. This shows how socially, it is easier to let something be than force a confrontation. People don't have knowledge about how useful a comment is to all of the readers (which would be necessary to comfortably delete something). In contrast, they only need knowledge that something was useful to themselves to justify adding it. The result is that some content becomes outdated and not selective enough.

The second criticism of the wiki has been its lack of consistency and a “professional look.” Many pages switch between first and third person. Different pages use different formatting techniques for the same purpose (e.g., headers versus italics). Some people sign comments, while others don't. Different writing styles and tones are used within the same page.

Although some people would prefer the wiki to look more professional, it is not clear that its unprofessional feel is critical. Most times when people came to the email list because they couldn't understand something on the wiki, the problem had to do with the specific wording of the content, not issues of consistency. However, it is possible that some visitors (especially those unfamiliar with the email list) don't give the wiki content a chance because they "judge the book by its cover."

\section{Participation and Community Roles}

\section{Wiki Work}

There are many ways that individuals contribute to the wiki. Members add content by creating new pages and adding to existing pages, edit existing content and page names, organize pages through adding hyperlinks, and delete content (e.g., spam). Interviews and content analysis reveal that much of the new content added to the wiki comes from the email list discussion. When information is transferred between the two it 
is often summarized and distilled before being entered into the wiki. Thus, the wiki encourages some new forms of work (e.g., distilling, organizing, re-writing) to occur; work that is different than that occurring on the email list. The work of distilling, organizing, and editing also requires a different set of skills than the work occurring on the email list. Although most people seem to prefer to contribute to the email conversation, some of the individuals I interviewed enjoyed contributing to the wiki far more.

Qualitative analysis of the "summary of change” descriptions (from the 14 wiki snapshots summarized in Figure 5.1) suggests that most contributions are minor in nature. Common examples include fixing a broken link or typo, removing spam, clarifying an ambiguous statement, and adding a small section to an existing page. This is corroborated by the data. For example, the average Bytes added per edit for all wiki pages (excluding SandBox and FrontPage) is 156 (just over 20 words).

On occasion, individuals make major contributions to the wiki. For example, a single member initially created the ThreeColumnLayouts page with dozens of entries with notes. Many of the Article pages were also begun as major projects. In a few cases, an individual has spent considerable effort reorganizing pages (e.g., editing the FrontPage; tagging Biography pages with a Category tag), although these are infrequent.

Contributions to a particular wiki page are often bursty in nature. This happens for a number of reasons. First, some individuals notice their own mistakes after making an edit (e.g., they notice a formatting error when viewing the recently edited page). Second, people following the RecentChanges page are more likely to visit (and potentially edit) a page that was just modified. Third, external events like the release of a new browser can lead to many edits to pages related to the browser. And finally, pages discussed on the email list are brought to the attention of numerous individuals at the same time, many of whom visit (and potentially edit) the page, especially when the author of the email message solicits such activity. Although edits are often bursty in nature, this does not 
suggest that after a particular burst of activity the page will remain the same inevitably, as evidenced by the periodic editing of many of the original wiki pages. In summary, wiki editing activity for a given page can typically be thought of as periods of drought interspersed with occasional downpours rather than a steady drizzle, although the type and popularity of page does seem to impact the climate.

\section{Community Roles}

There are several distinct roles played by individuals who use and contribute to the wiki. The discussion of these roles is primarily based on interviews, as data on wiki usage and contribution patterns was imperfect (e.g., few members sign their name when making edits and no person-level detail was available from the wiki log files). The analysis of the RecentChanges page from the 14 wiki snapshots suggests that contributors to the wiki can be numbered in the hundreds. This is in comparison to the tens of thousands of unique visitors of the wiki each month (see Figure 5.2). Active wiki participants who regularly monitor the RecentChanges page suggest that there are a handful of active contributors and many infrequent contributors. However, even they have trouble knowing who is contributing because many people don’t sign in when making edits. Chapter 6 will discuss how the wiki is used by various email list members. Here I focus on the social roles related to wiki contributions.

Interviews of several of the most active wiki contributors suggest that there are two distinct groups of active participants. This was confirmed through content analysis of wiki pages. The first group can be thought of as 'Bridge Builders' because of their active role in the email list and wiki. These individuals include several administrators and prominent members of the email list. They are CSS experts, but have little prior experience using wikis. Bridge Builders transfer information from the email list to the wiki after distilling it, and often encourage others to do so as well. Some of them contribute to numerous wiki pages, while a handful each focus on a particular page or 
two that they actively maintain (e.g., see Article genre section above). Bridge Builders also refer others to the wiki in their email list conversations. Many of these individuals have contributed to the email list and wiki for several years. Two of those that I interviewed mentioned having recently stopped their participation in the email list (due to the time it took to read all the messages), although they still occasionally contribute to the wiki (e.g., to maintain sites of interest).

The other group of active wiki contributors can be thought of as 'Wiki Experts.' These are individuals who have significant prior experience using other wikis (e.g., Wikipedia, MeatballWiki, Twiki) and rarely, if ever, post to or read the current email list messages. However, they often use the email list archives. When Wiki Experts do post to the list it is likely to be about the wiki. These individuals are more likely to sign in when making edits and to have longer Biography pages with personal opinions. They are also more likely to use the wiki as a means of communicating with other members through signed comments. Some Wiki Experts have tried to implement practices common to other wikis (e.g., the use of Categories) with mixed success. These members seem to come and go more rapidly than Bridge Builders, although their impact on the wiki is noticeable long after they leave.

In addition to the active wiki contributors, a few individuals contribute more sporadically. One recognizable group of infrequent contributors self-identifies themselves as "spam hunters.” These individuals actively monitor the RecentChanges page (some use the RSS feature) and remove spam when it is posted to a page. Some of these individuals feel like their level of CSS expertise is not sufficient to help others. They remove spam as a way of giving back to the community (see Biography genre section for relevant quotes).

\section{Conclusions}


There is far less interaction on the css-d wiki than in other popular wikis (e.g., WikiWikiWeb, Wikipedia). Rather than serving as a site for interaction, it has primarily been used as a knowledge repository where items of lasting value are stored. This was the original intent of the wiki (as evidenced in the original wiki policy pages) and has largely remained the same. The technological features of the wiki (its ability to collaboratively edit and structure page content, organize pages, and hide past page versions) have supported this activity well. The technology has also facilitated the creation of new genres that were not practical to have in the email list such as the Annotated Links, Debate, and Article genres.

The widespread use of the wiki in the ongoing conversation (see Chapter 6) and by the public at large (see Figure 5.2) suggests that this content is valuable, although some of it is outdated and not as consistent and professional as some would like. It seems clear that the wiki could be improved by assigning a "clean up crew" or wiki administrator whose could help delete or update outdated material, organize content, and format pages consistently (see Chapter 7 for further discussion). Indeed, two interviewees suggested this idea.

It is telling that even though members recognized both the problem and a possible solution, the solution has not yet been tried. Unlike a novel, which is typically read as a whole, the css-d wiki is a collection of very different pages that typically stand on their own. Thus, my use of a well-maintained wiki page is not inhibited because other wiki pages are outdated. This may contribute to the members feeling that it is just not worth the effort to clean up the entire wiki, which (even with its flaws) seems to be working well enough. Members have never thought of the wiki as a comprehensive, authoritative manual on CSS. Rather, it has been viewed as a collection of documents designed to be useful to the community when answering frequently asked questions and closing down Holy Wars. These basic functions can be met by referencing the most important pages (see next chapter) which are typically well-maintained, without needing to make sure that 
all of the other pages are perfectly maintained and professional looking. Although they may be right about the wiki being good enough to meet the needs of the conversation, it is possible that outsiders visiting wiki pages that are not well maintained may assume that the entire community is behind the times. This suggests that it may be important to establish the role of wiki administrators or clean up crew members to make sure that newcomers and outsiders are more inclined to respect and join the community.

Like the content in the email list, the content on the wiki is highly practical to real-world problems. This is because of its close association with the email list, which is focused on answering practical CSS questions. Although some of the practical suggestions are lost in the distillation process, most pages have transformed the specific suggestions and considerations into more general ones. Instead of simply defining a CSS technique, the Definition pages often include warnings about the use of the technique. Instead of simply listing all of the ThreeColumnLayouts, helpful annotations about the browsers they support and the techniques they rely upon are provided. Instead of a drawn out debate about font sizing, the font size Debate pages include summarized lists of practical issues to consider.

Members recognize the unique value provided by the wiki because of its practical nature. They talk about it being the most comprehensive storehouse of "tips and tricks" on the web. Although this is useful to the email list members, it may be of even more value to people who would not be willing to search the email archives or register for the list. Thus, the wiki helps bring to light all of the practical pieces of wisdom that come out of the highly contextualized discussions in a help-based email list.

One of the most useful characteristics of the wiki is its ability to aggregate information from many different people. Several of the most actively edited and popular wiki pages are Annotated Links pages. The wiki's ability to structure content through tables and bulleted lists made it possible to aggregate links and add annotations to help with comparison. Pages like the ThreeColumnLayout page are not possible to create or 
maintain in an email list. Furthermore, the easily editable nature of the wiki encouraged micro-contributions, enabling pages to include content from numerous authors. This is especially important when trying to aggregate knowledge that is disparate and rapidly changing.

The How To pages also nearly always aggregated various techniques into a single page. Interviewees discussed how these pages were useful when looking for a specific technique, because they helped people decide which technique is most applicable to their particular situation. Some members mentioned using the wiki as a "collection of bookmarks” because it did such a good job of collecting them into one place. Some of the Debate pages like UsingFontSize also demonstrate the usefulness of the wiki's structured formatting in helping aggregate and compare various arguments.

\section{Email List and Wiki Comparison}

Although the topics are relatively similar on the email list and wiki, the form they take is quite different and complementary. Most of the web design content pages include information in a summarized and de-contextualized form intended to be useful to many people. Several interviewees contrasted this with the personalized, interactive nature of the email list as in the following interview quotes from different interviewees:

- The Wiki is a filter of the discussion so it's after people have talked about it for a few hours, days, weeks. You put the final true stuff on the Wiki. So you filter out all the noise and you get the answers in the Wiki. So that's my view of it. That's why I go to the Wiki first 'cause I don't have to wade through thousands of emails, threads and discussions, back and forth bantering. I can just go to the Wiki and get the answer that I'm looking for.

- The purpose of the list is more to solve a problem with CSS someone has encountered while designing a real page. The wiki generalizes the problem and the workaround. 
Members refer to “distilling," “summarizing,” and “collating” email list threads into wiki pages. The following wiki page content taken from the ConstantsInCss page is illustrative:

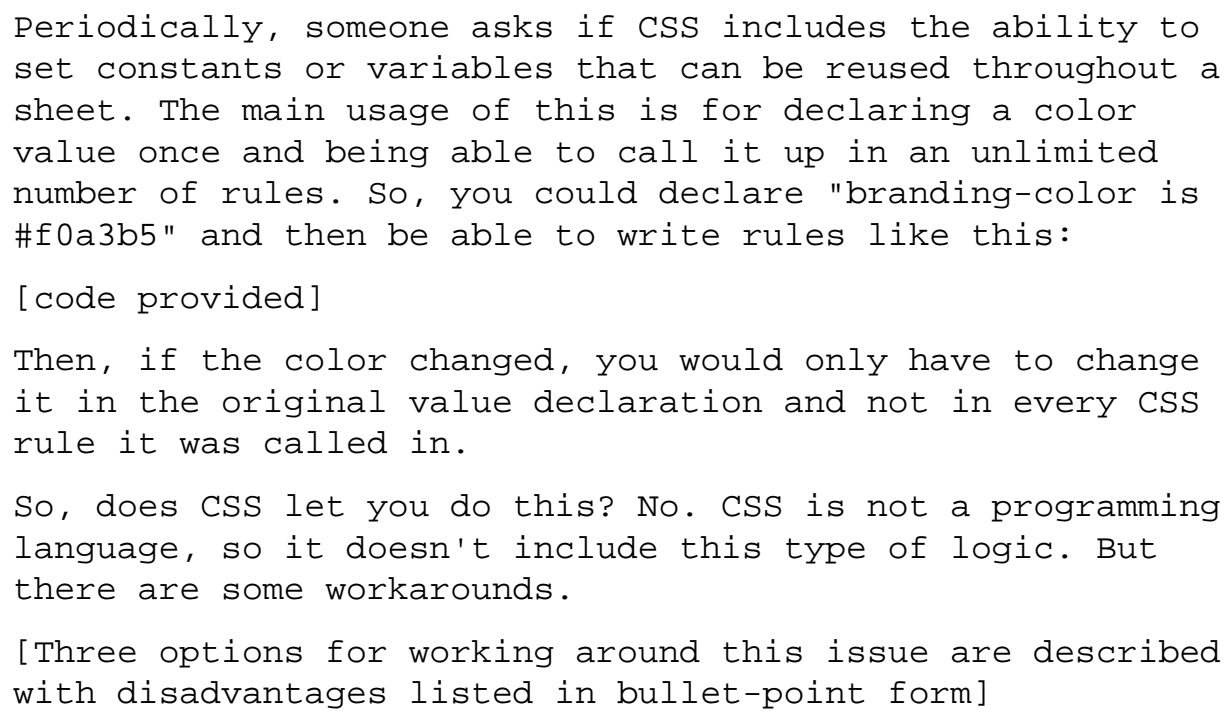

In this case, the introductory paragraph describes the issue in a generalized form (i.e., does CSS include the ability to set constants?). It also provides an example of when this issue may arise (i.e., when declaring a color value). This particular example was, in fact, the original question asked to the email list that led to the creation of this page. None of the information about the questioner's particular situation and needs (which was included in the email thread) or the redundant and unhelpful replies it received were added to the wiki. Instead, only a brief summary of the generalized issue with a summarized version of the "main" need that led to the issue were included, along with summarized versions of various techniques and their disadvantages. Thus, the original context was not entirely removed, although it was de-emphasized and recast in more general terms.

The de-emphasizing of the context is important because it allows for write-ups to apply to many different, but related situations. The summarization is important because it 
makes the information more findable and easier to digest. Combined with the modular format of the wiki with its persistent URLs, the content of the wiki is ideal for reuse.

Unfortunately, the decontextualization can also make pages difficult to find and even when found they can be difficult to apply to particular cases. For example, some people would not think to look at a page titled ConstantsInCss to help them overcome the problem of changing colors in their CSS file (see Chapter 6 for a discussion of this issue). And even if someone did find the page, they may not know which suggested method is best for their own situation, because it may require a knowledge of CSS that they do not have.

Fortunately, the strengths of the email list conversation help to compensate for these limitations of the wiki repository. Individuals can explain their own problems in a highly contextualized manner (which is easiest for them to do) and experts on the list can fairly easily point them to resources (e.g., wiki pages) pertinent to their situation. As we saw in Chapter 4, this is especially helpful when list members follow the suggested practices and help explain how the resource applies to their situation.

In the following chapter I will further explore the complementarities between the email list and wiki and the processes, community roles, and activities necessary for their existence. 


\section{CHAPTER 6}

\section{CSS-D EMAIL LIST \& WIKI COMPLEMENTARITIES}

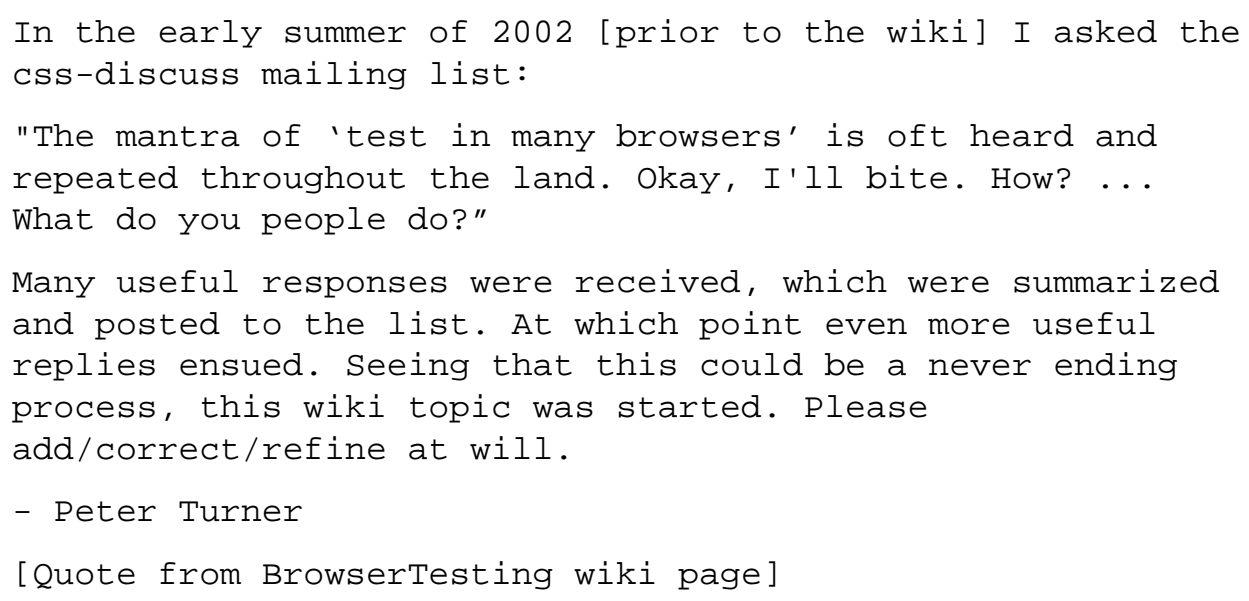

Although the wiki and email list are not technically linked together, they are socially coupled quite closely. They share many of the same participants and the content from each of them influences the content of the other. In this chapter I describe how the two are coupled and the implications of this coupling on the information that is shared within the community. I argue that like a symbiotic relationship, each benefits from the other creating a situation where the sum is greater than the individual parts.

Before looking at the specific ways that the email list and wiki influence each other, we need to understand the social practices and activities that link the two together. The first two sections of this chapter describe the social roles and referencing behavior that connect the two. Next, I will discuss how each influences the other, ending with conclusions.

- Coupling of Email List and Wiki

- Coupling Behavior

- Community Roles \& Activities 
- Email List $\rightarrow$ Wiki

- Wiki $\rightarrow$ Email List

- Conclusions

\section{Coupling of Email List and Wiki}

\section{Coupling Behaviors}

There are many social actions used by members to connect the email list and wiki. For example, members refine questions based on content from the wiki, they refer to wiki pages when answering questions in the email list, and they suggest that content be added from the email list to the wiki. This section describes these and other social actions that are central to the coupling of the two. I first provide an example of this coupling by tracing the history of a particular wiki page (ConstantsInCss) and the related email list messages. Quantitative findings follow.

\section{ConstantsInCss Example}

One frequent question that arises on the email list is whether or not constants can be defined in CSS. The answer is “no," but there are many ways around the issue. This question generally comes up when website developers want to change the color scheme of a site and are frustrated that they must change the color in a number of places throughout the CSS code. The following thread shows how the discussion of this frequent question led to the initial creation of the wiki page ChangingTheColorScheme.

January 2005 Thread: Definition of Constants?

1st of 10 messages in Thread; Sent by Adam in early 2005

$\mathrm{Hi}$ everybody,

I am about four weeks into cSS now, and I have the following question:

Is it possible to define constants/shortcuts/names/... for colors (or any other kinds of values that a property might take? 


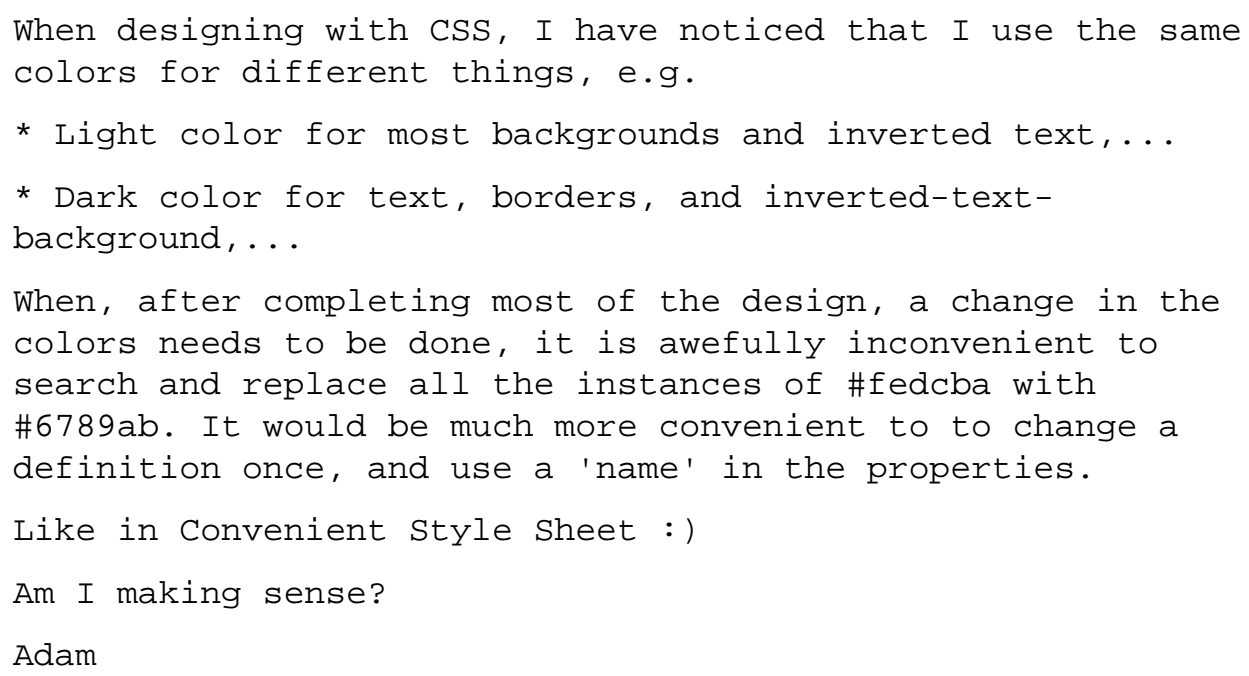

As is often the case, the questioner, Adam brings the question to the email list because he is dealing with some current problem.

Messages 2-7 of this thread state that there is no way to define constants using CSS, and then go on to propose various workarounds (e.g., using a scripting language such as PHP, adding CSS comments to aid with "find and replace”). As is typical, there are multiple ways of dealing with the problem, each with its own set of potential costs and benefits. In this case, no more than one technique was suggested by each author. Furthermore, there is often not universal agreement about recommended techniques. For example, one member warned others away from another member's recommendation: “ouch. I wouldn’t do that...IMHO, too much work just to have pseudo-constants.”

The final two messages in the thread relate to transferring the knowledge to the wiki.
9th of 10 messages in Thread; Sent by Beth (an administrator) about 23 hours after original post

This has become an FAQ, so I encourage one of you generous souls to start a wiki page for it. :-) Then send the URL to the list, and others can help you fill in the holes.

Thanks, Beth [one of the email list administrators] 
When questions, such as this one, frequently occur in the email list, it signals that the topic is of interest to many people. Core community members like Beth who actively read most email messages are able to recognize these frequent questions. Because Beth also knows the wiki quite well, she realizes that there is not currently a page on the topic and recommends that some "generous soul" start one, and then tell the list so they can help “fill in the holes.” Beth's status as a list administrator and active participant make her suggestion acceptable. This is in contrast to other instances where newcomers propose that old-timers add something to the list, only to receive a brief "lecture" about how anyone can edit the wiki and the newcomer should take the initiative.

The prior message motivates Adam (the original questioner) to create a new wiki page called ChangingTheColorScheme. This was also added to the wiki’s FrontPage under the "Tips and Tricks" subcategory with the following description: "Changing the color-scheme after the design has been mostly completed.” The wiki page was introduced (as suggested by Beth) in the following email.

10 th of 10 messages in Thread

Sent by Adam about 32 hours after the original message

$>$ This has become an FAQ, so I encourage one of you generous

>souls to start a wiki page for it. :-)

Like this? [URL to ChangingTheColorScheme wiki page]

$>$ Then send the URL to the list,

Done :)

So, everybody who gave advice: Please take my summary on the wiki as a 'Thank you',

Adam

This thread demonstrates how the email list can influence the creation of wiki content and the social roles and actions important for this to occur. The topic for the wiki page was suggested by its repetition in the email list, a signal of its importance to many people. This required a member to actively read most messages to recognize the 
frequency of the topic. The content for the wiki page was also generated in the email list, although it had not yet been distilled. This content was also peer reviewed in the email discussion. This influenced the quality of the wiki content, as evidenced by the fact that the rejected technique was not included. Thus, the wiki page included (and excluded) ideas from several list members after they had been publicly vetted, even though it was added by only one individual. Finally, the motivation for performing the work of distilling the thread into a wiki page was provided because of the email list conversation (i.e., the member who added it did so as a "thank you" to the list). He also did it as a response to a publicly offered suggestion by a core community member and administrator.

About a month later a new thread was started by a newcomer to the list asking the same question. The question was followed by a few suggestions and some back-and-forth discussion about the accuracy of a claim made in one of them regarding a PHP script. The following message was sent by Carl (one of the administrators).

February 2005 Thread: Constants in CSS

7 th out of 8 messages in thread

Sent by Carl about one month after prior thread

$>$ I looked through the archives (briefly) but couldn't find >anything on this.

[Email Archive URL, Subject, and Date of the prior thread included]

In fact, last time round, fellow list moderator, Beth asked for the info to be written up on the wiki since it's a perennial "favourite"

[URL for ChangingTheColorscheme included]

If anyone wants to improve on that, make the name better, or anything else they can think of, please feel free... >Does anyone know why cSS doesn't have the ability to define a >constant, Yes, the W3C's working group on cSS. Ask them. (actually don't - search their archives first ;)

[URL to W3C email archives] 


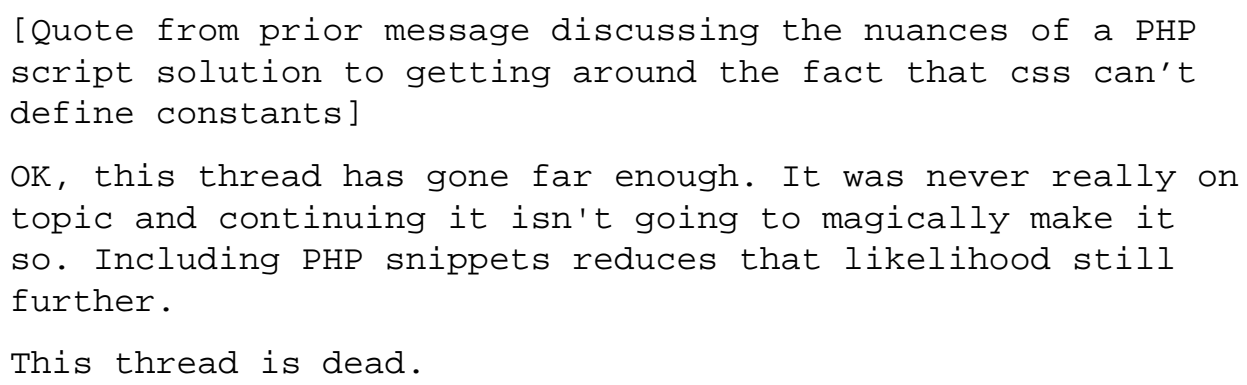

The primary purpose of this Administrative message was to shut down an offtopic thread. In contrast to the former thread that discussed several practical workarounds (and was considered on-topic), this thread speculated on whether or not future CSS releases would include this capability and debated about the details of a non-CSS solution. A secondary purpose was likely to remind list members to check the email archives and wiki more thoroughly before posting questions - although Carl recognizes that the wiki page (with a title about color schemes not css constants) may not have been very easy to find. Carl's link to the wiki page serves several purposes. First, it provides potentially useful content to the question asker that is in a reusable format. Second, it provides a way of shutting down an off-topic discussion without losing the potentially useful information being discussed. Finally, it encourages list members to incrementally improve the wiki page.

The topic has continued to come up on the email list periodically and has led to further refinements of the page. Two external links that were announced to the email list (and related to the topic) were added to the wiki page. The page was renamed (nearly a year after the original suggestion) by Beth, who still remembered Carl's implicit challenge to "make the name better." Beth announced this change to the list in a message titled "FYI: CSS Constants wiki page updated” and encouraged others to "explain” and "flesh out" the scripting language suggestions outlined in "option 3" since that was not one of her areas of expertise. A handful of changes were made to the page in the "option 3” section, although not right away. 
This example shows how the wiki page was useful in the discussion (to help answer questions and shut down off-topic discussions). It also shows how this use led to its maintenance over time. For example, Carl recognized that the original page name (ChangingTheColorScheme) did not reflect the frequently asked question he was dealing with and suggested its modification (which eventually happened). In another message, Beth announced that she had changed the page title and used that as an opportunity to request that others clean up the "option 3" section.

In summary, the ConstantsInCss example shows us how members couple the email list and wiki and hints at some of the benefits of this coupling. We will now look more broadly at this coupling behavior within the list.

\section{Wiki Referencing Behavior}

One of the primary ways to better understand how the email list and wiki are related to one another is to examine how the wiki is referenced in the email list conversation. This helps us understand the situations where the wiki is most useful to the conversation. It also helps us see how the email list discussion can influence the wiki.

Of the 45,580 total messages sent between January 2003 and April 2005, 1,787 (3.9\%) referenced the wiki, an average of 2 per day. ${ }^{4}$ I will refer to these messages as “wiki emails.” Although this is a relatively small percentage, they have had a significant effect on the wiki content and conversation. The percentage of these wiki emails has increased over time (see Figure 6.1), with an average of 5.5\% of emails referencing the wiki during the final 6 months of the dataset. Interviews and content analysis also suggest

${ }^{4}$ These messages were identified by searching for the wiki URL, as well as the word "wiki". Content analysis of these messages (presented later in this chapter) revealed that a small percentage of these messages refer to wikis other than the css-d wiki. See Chapter 3 for details of the coding methods. 
that it is common for individuals to provide pointers to the wiki in private email messages sent to list members.

Figure 6.1: Percent of All css-d List Messages Referencing the Wiki

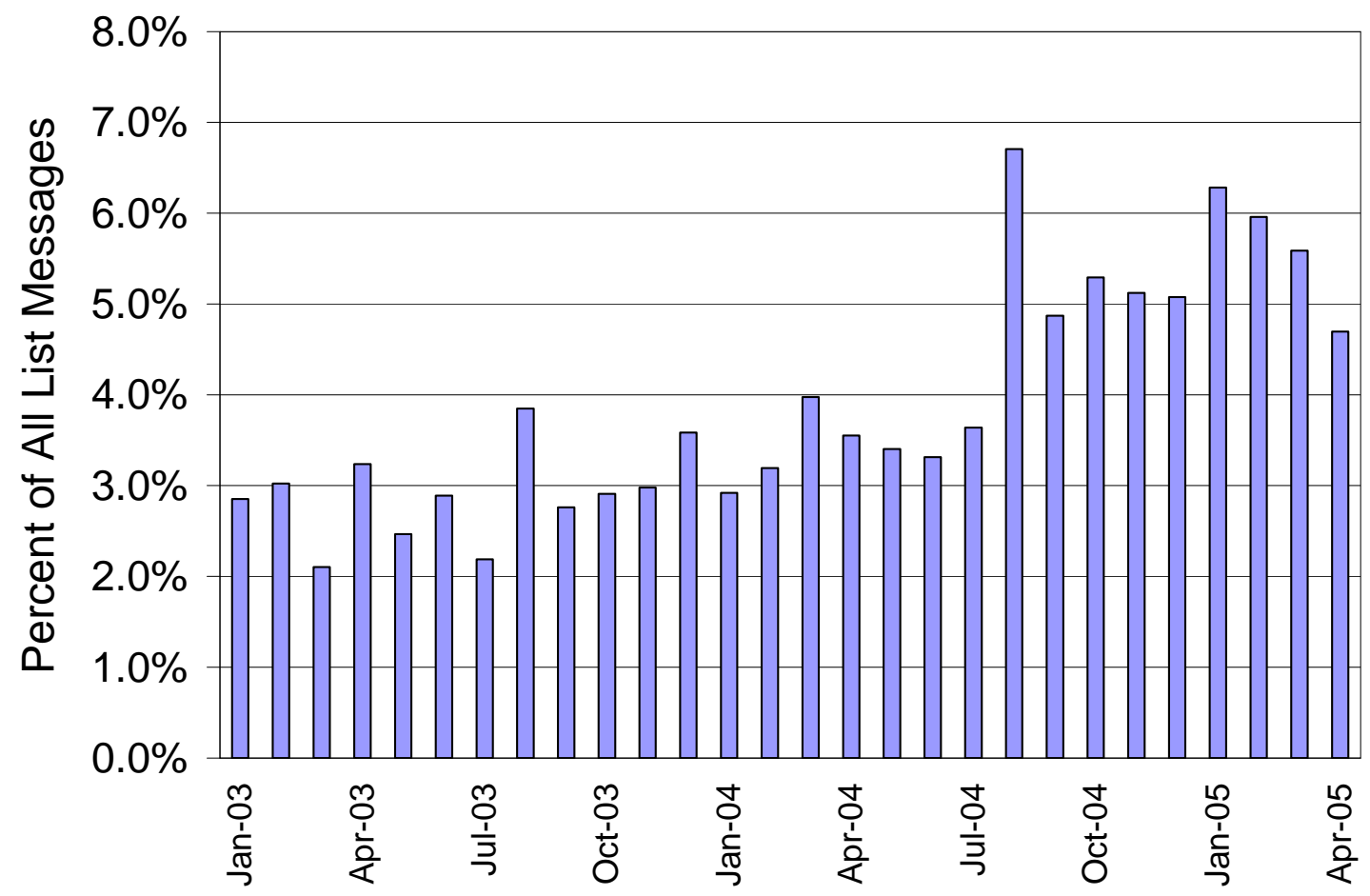

Wiki referencers are numerous (530 or $14 \%$ of all posters). The distribution of wiki emails sent by each individual is highly skewed, similar to the distribution of total emails sent by individuals. The top wiki referencer (who is also the most frequent list contributor) posted 152 wiki emails (8\% of all wiki emails) and the top 28 wiki referencers (representing 5.3\% of all wiki referencers) accounted for $50 \%$ of all wiki emails (Gini coefficient of 0.62). The majority of the wiki referencers (61\%) only referenced the wiki once.

Wiki referencers are a heterogeneous group including members of all participation levels in the email list (see Figure 5.2). Frequent posters are more likely to reference the wiki. For example, the most frequent posters, defined as those who account for $20 \%$ of all email list messages, posted $31.6 \%$ of all wiki emails. However, infrequent 
posters still reference the wiki regularly. The least frequent posters, defined as those who account for $20 \%$ of all list messages, contributed only $12.5 \%$ of all wiki emails. Thus, although the wiki is referenced more often by the most active members, it is still referenced considerably from members all across the spectrum of participation. The wiki's widespread use suggest that the wiki is of value to all of the email list community roles described in Chapter 4 including core members (who are frequent posters), intermittent members (who are moderately posters), and newcomers (who are infrequent posters).

Figure 6.2: Percent of All css-d Emails vs. Wiki Emails by Posting Frequency

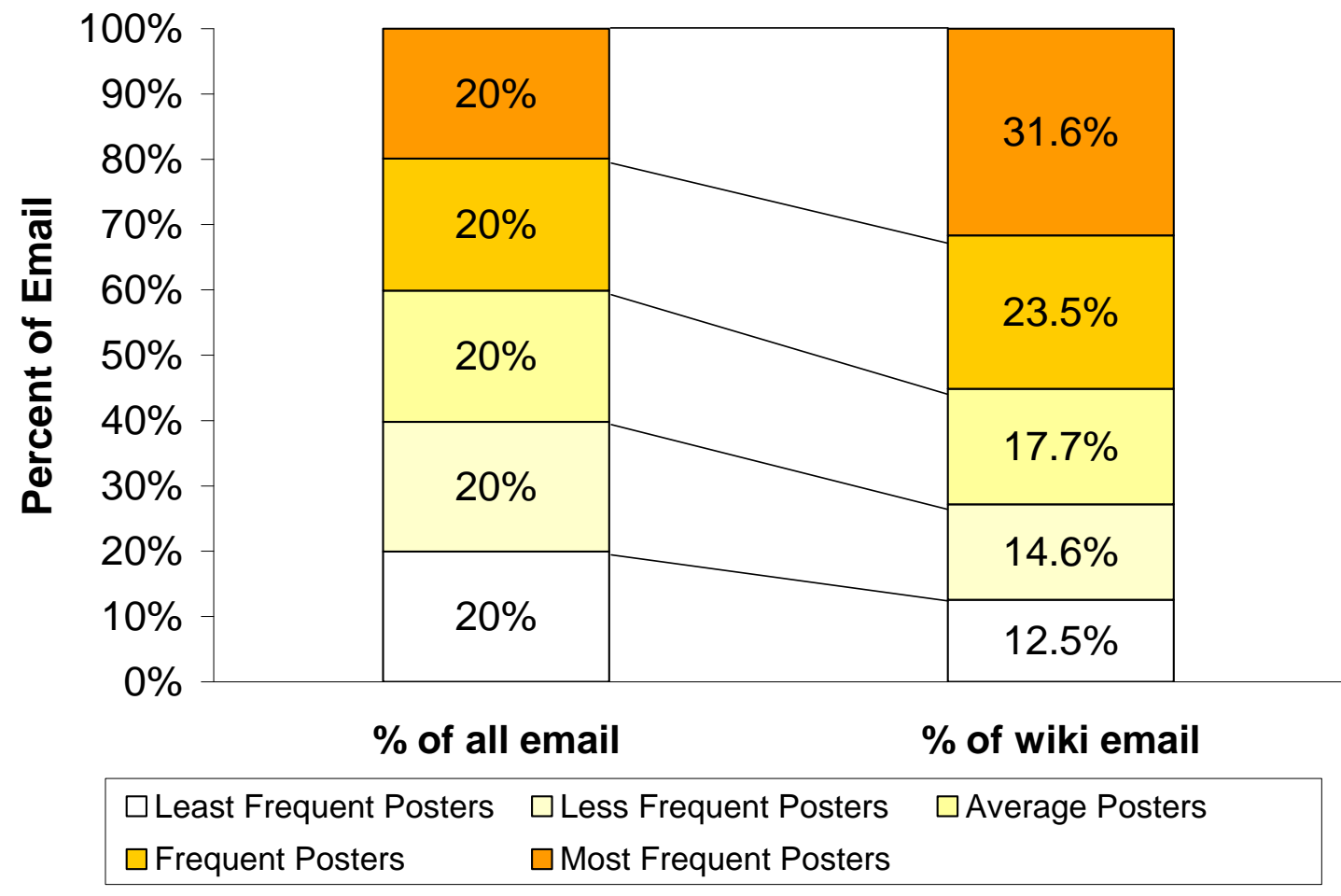

\section{How and Why is the Wiki Referenced?}

In most cases, links to specific wiki pages are provided, as opposed to general pointers to the wiki. Of the 1,787 wiki emails, 1,117 (63\%) of them included at least one URL linking to a specific wiki page other than the wiki’s home page (i.e., FrontPage). 
Many others suggested search terms or topics to look for on the wiki. Referencing specific wiki pages is important because signals about the wiki content's quality and relevance are tied to specific pages. Not only are readers (i.e., questioners) more likely to visit the appropriate page, those inserting the links (i.e., answerers) nearly always visit the page in order to copy the sometimes non-intuitive URLs and make sure the content is still relevant. Interviews revealed that this occasionally leads the question answerers to edit the visited wiki page.

The wiki is referenced for a number of reasons. Table 6.1 shows the major reasons that the wiki was referenced, as well as the reasons the email archive was referenced, for comparison (see Appendix 2 for a full description of the categories and Chapter 3 for a discussion of the message coding procedures). Note that not all 1,787 wiki emails used in the prior analysis were analyzed, as some of them referenced the wiki only in quoted material or referred to a different wiki leaving a total of 1,511 (see Chapter 3 for details). Cohen kappa values were above 0.80 except where noted with an asterisk in Table 6.1.

The groups in Table 6.1 are not mutually exclusive: a single message may include a reference to the wiki to help answer a question, as well as a discussion of prior edits made to the page. It is also important to recognize that the purpose of the email messages is not being coded. Rather, the coding is of the reason for referencing the wiki. On average, a single wiki message was classified into 1.16 categories and a single archive message into 1.13 messages.

Table 6.1: Reasons for Referencing the css-d Wiki \& Email List Archives

\begin{tabular}{|l|l|l|l|l|}
\hline Reference Reason & Wiki & Archive & Wiki \% & Archive \% \\
\hline Answer question or educate & 917 & 158 & $61 \%$ & $52 \%$ \\
\hline Help ask question or justify a post & 248 & 60 & $16 \%$ & $20 \%$ \\
\hline Meta discussion about its proper use & 82 & $25 *$ & $5 \%$ & $8 \%$ \\
\hline
\end{tabular}




\begin{tabular}{|l|l|l|l|l|}
\hline Offload discussion & 74 & $3^{*}$ & $5 \%$ & $1 \%$ \\
\hline Logistical issues (e.g., usability) & 44 & 51 & $3 \%$ & $17 \%$ \\
\hline Tell others to search before posting & 32 & $10 *$ & $2 \%$ & $3 \%$ \\
\hline Other email list procedures & 23 & 1 & $2 \%$ & $0 \%$ \\
\hline Give thanks & 77 & 12 & $5 \%$ & $4 \%$ \\
\hline Discuss a prior edit (wiki only) & 66 & $\mathrm{n} / \mathrm{a}$ & $4 \%$ & $\mathrm{n} / \mathrm{a}$ \\
\hline Discuss a future edit (wiki only) & 152 & $\mathrm{n} / \mathrm{a}$ & $10 \%$ & $\mathrm{n} / \mathrm{a}$ \\
\hline Refer to prior thread (archive only) & $\mathrm{n} / \mathrm{a}$ & 12 & $\mathrm{n} / \mathrm{a}$ & $4 \%$ \\
\hline Other & $43^{*}$ & $10 *$ & $3 \%$ & $3 \%$ \\
\hline All Messages & 1,511 & 302 & & \\
\hline
\end{tabular}

These numbers are discussed throughout this chapter. I emphasize only two general trends here. First, the wiki is referenced five times more often than the email archives. In contrast, the archives are visited 3 times more often than the wiki (8 million times versus 2.5 million times between Oct 2004 and Jan 2007). This suggests that the wiki is particularly useful as a community resource, while both resources (and especially the comprehensive archive with over 90,000 messages) are useful to outsiders. Second, both resources are primarily used in the community discussion to help ask and answer questions, although they are also used considerably for administrative purposes.

\section{What Wiki Pages are Referenced?}

Because some wiki emails reference multiple wiki pages, there were more specific wiki references $(1,248)$ than wiki emails that link to at least one specific wiki page $(1,117)$. In total, 112 unique wiki pages were referenced. These pages accounted for $60 \%$ of all wiki content as measured in bytes (using February 2005 wiki data since it is in the time period of the email messages). The number of times each wiki page was referenced is a long-tailed distribution (see Table 6.2 for the most referenced 13 pages that account for half of all references) with 15 pages being referenced only once (Gini 
coefficient 0.61 ). It is also apparent from Table 6.2 that a relatively large number of individuals link to the most referenced pages. For example, over half of the references to the BoxModelHack page were from different people.

Table 6.2: Most Referenced css-d Wiki Pages

\begin{tabular}{|l|l|l|l|l|l|}
\hline Page Name & $\begin{array}{l}\text { Times } \\
\text { Referenced }\end{array}$ & $\begin{array}{l}\text { Cumulative } \\
\text { References }\end{array}$ & $\begin{array}{l}\text { Unique } \\
\text { Referencers }\end{array}$ & $\begin{array}{l}\text { May 06 } \\
\text { Size (Bytes) }\end{array}$ & $\begin{array}{l}\text { May 06 Page } \\
\text { Edits }\end{array}$ \\
\hline ThreeColumnLayouts & 111 & $8.9 \%$ & 40 & 12,053 & 121 \\
\hline BoxModelHack & 87 & $15.9 \%$ & 46 & 15,027 & 94 \\
\hline PrintStylesheets & 71 & $21.6 \%$ & 22 & 16,425 & 53 \\
\hline AnyColumnLongest & 60 & $26.4 \%$ & 28 & 4,782 & 29 \\
\hline FooterInfo & 47 & $30.1 \%$ & 25 & 2,442 & 44 \\
\hline CenteringBlockElement & 41 & $33.4 \%$ & 20 & 6,431 & 43 \\
\hline RenderingMode & 40 & $36.6 \%$ & 21 & 3,717 & 40 \\
\hline OffTopic & 32 & $39.2 \%$ & 14 & 2,285 & 21 \\
\hline ClassesVsIds & 29 & $41.5 \%$ & 19 & 6,624 & 25 \\
\hline ScreenreaderVisibility & 28 & $43.8 \%$ & 14 & 14,272 & 49 \\
\hline FontSize & 27 & $45.9 \%$ & 18 & 4,077 & 40 \\
\hline CssEditors & 25 & $47.9 \%$ & 14 & 8,561 & 110 \\
\hline CssHacks* & 24 & $49.8 \%$ & 18 & 47 (3,866) & 75 (45) \\
\hline $\begin{array}{l}\text { *CssHacks was changed to CssHack during this time period. Numbers in parentheses indicate values for } \\
\text { CssHack. }\end{array}$ & \multicolumn{5}{l}{} \\
\hline
\end{tabular}

The wiki pages that were referenced in the list are not representative of the overall distribution of wiki pages as shown in Table 5.1. The vast majority of referenced pages linked to 'Web Design' content pages (91\%). These referenced pages represented $40 \%$ of all Web Design wiki pages and 74\% of the total Bytes of information classified in that group. Six of the 9 'Email List' pages (e.g., OffTopic, CssDiscussListHeaders) were referenced. Only 4 of the 67 pages that made up the 'Site Check,' 'Category,' and 'Wiki Admin’ page types were referenced, and no pages classified as ‘Biography' or 'Spam/Junk' were referenced. 
Interviews and content analysis suggest that individuals who reference a particular page are likely to have edited the page in the past. One important example of this is when individuals create a new wiki page and introduce it to the list (something that has happened over 20 times by more than 11 different individuals). As discussed in Chapter 5, several wiki pages (e.g., PrintStylesheets, ScreenreaderVisibility, StyleInEmail) have a self-identified editor who maintains the page and refers to it in the email list when applicable. Although the initial references to these pages come from their authors/editors, other members often catch on and reference them as well.

\section{Community Roles \& Coupling Activities}

There is a significant overlap in membership between the email list and wiki. This overlap is important in coupling the two together. Members need to be familiar with the wiki content in order to reference it in the email list. Likewise, members need to know what topics are discussed in the email list to know what wiki pages would be of most benefit. There are a few specific social roles that have helped couple the email list and wiki in meaningful ways. Chapters 4 and 5 discussed social roles related to the email list and wiki respectively. Here I highlight those social roles that have the most significant impact on coupling the email list and repository.

\section{Bridge Builders}

In Chapter 5 I described Bridge Builders, or individuals who transfer information between the email list and wiki and encourage others to do so. Here I provide more details on their particular actions and separate them into two groups. Although some individuals fit into both groups, other members fit one or the other, and the separation is analytically meaningful.

Wiki Advocates - these are individuals who help promote the wiki within the email list, as well as encourage list members to improve the wiki. Some of these 
members are admins, although not all are. Having list administrators play this role seems to work particularly well, since they have some authority when suggesting that others perform a certain way (e.g., add to the wiki; reference the wiki more often; search the wiki before posting). Other core members often share a similar authority, even if it is not explicitly provided. However, even a few intermittent posters speak with authority concerning the wiki because they have contributed to it so much.

Wiki Advocates are among the most active wiki referencers. They reference many different wiki pages and suggest that others reference the wiki as well. They also point out areas where the wiki could be improved. They may suggest new pages (as in the ConstantsInCss example), or suggest edits to an existing page (as also happened in the ConstantsInCss example). A typical example sent from a Wiki Advocate in reply to a question about “dot leaders" is shown below:

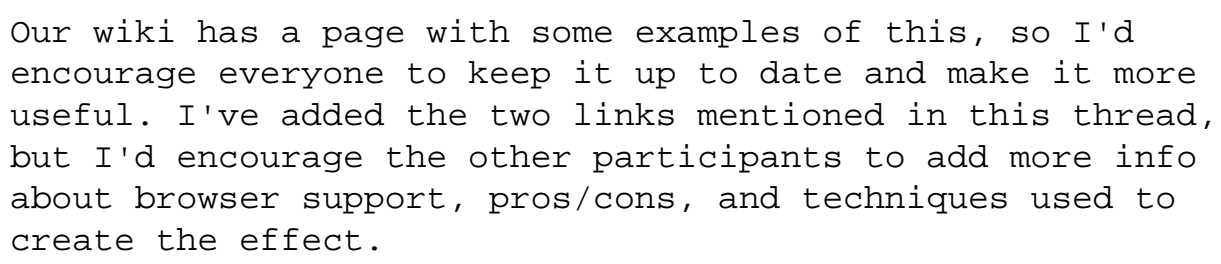

As is typical, the Wiki Advocate leads by example ("I’ve added the two links...”) and provides specific suggestions on how the wiki can be improved ("add more content about...”). They also thank other members for their contributions to the wiki, providing public recognition of others efforts. Wiki Advocates thus play an important role in transferring information between the two resources and motivating others to do so as well.

Wiki Page Editors - these are individuals who take a special interest in a particular topic, create a wiki page on it, reference the page in the email discussion, and update the page based on the email discussion. Typically, these pages are comprehensive 
Article pages on css sub-topics (see Chapter 5). The following email sent by a Wiki Page Editor is telling:

Folks,

I started a thread on this subject recently. Thanks to all those who replied. As a result of the interest shown, I have created a page on the Wiki for more permanent reference: -

[DiagnosticCss wiki URL]

It includes a link to the example diagnostic style sheet which I originally offered. I have since made an improvement to the header $\langle\mathrm{hx}\rangle$ diagnostic and have improved some of the notes and explanations.

I would really appreciate feedback (on-list or off-list) from people actually using it (what does it tell you about your own sites and others? how does it help you? have you modded it?).

Experienced listers who use the technique to diagnose other people's problems (it doesn't solve everything of course!) might like to refer to the Wiki page in their on-list replies. This will raise awareness of the technique and enable others to solve some of their own problems in future.

My continuing thanks to Brandon Hall, whose articles gave me the original inspiration.

[signature]

This example shows how the Wiki Page Editor originally collected ideas (and assessed interest) from the email list and then created a page on the topic. She then encouraged members to provide feedback on the wiki page content. Although not mentioned here, feedback that was received was added to the page by the Wiki Page Editor. Finally, she suggested that members reference the page in the email list discussion in order to "raise awareness of the technique and enable others to solve some of their own problems in future.” The Wiki Page Editor followed her own suggestion by referencing the page several times in the future. As is also typical, the Wiki Page Editor wrote the wiki page in first person and identified herself as the author, suggesting that people contact her with any suggestions for the page. Other wiki pages (e.g., 
ScreenreaderVisibility, PrintStylesheets, StyleInEmail, ProgressiveEnhancements) follow a nearly identical pattern.

\section{How the Email List Influences the Wiki}

Now that we have an overall framework for understanding how the email list and wiki are coupled, it is possible to look directly at the ways in which the email list and wiki influence each other. In this section I focus on how the email list influenced the wiki and the social and technical factors that underlie its successes. Specifically, I claim that the email list helps the wiki by:

- providing a foundation on which to build,

- motivating contributions,

- $\quad$ suggesting topics

- providing and reviewing content, and

- identifying problems with wiki content (or lack of content).

\section{Providing a Foundation on which to Build}

The existing foundation of the email list was immensely important to the initial success of the wiki. Indeed, when asked how the wiki would be different without the email list, one prominent list member suggested that the wiki would not exist because it is focused on a "fairly narrow technical topic." In other words, the email list helped gather a critical mass of contributors in a situation where it would be difficult to gather otherwise. People are drawn to the email list because they can have their immediate needs met. They often stick around because they learn from overhearing others' contributions and because of the sense of community and friendships they develop. Thus, the email list served as the community base, which enabled the wiki to be introduced as a worthwhile community project with all of the backing that implies. In other words, the critical mass of wiki 
contributors needed to assemble for reasons other than to simply create a useful repository.

The email list provided more than just a critical mass of potential wiki contributors. It also provided an existing social structure that helped focus attention and establish norms around the wiki. For example, the backing of the email list administrators and core members was important to the success of the wiki. Not only did they provide some of the initial content, they also advocated the use of the wiki on the email list. The following reply message was sent by a well-respected, core email list participant only a few months after the wiki was rolled out:

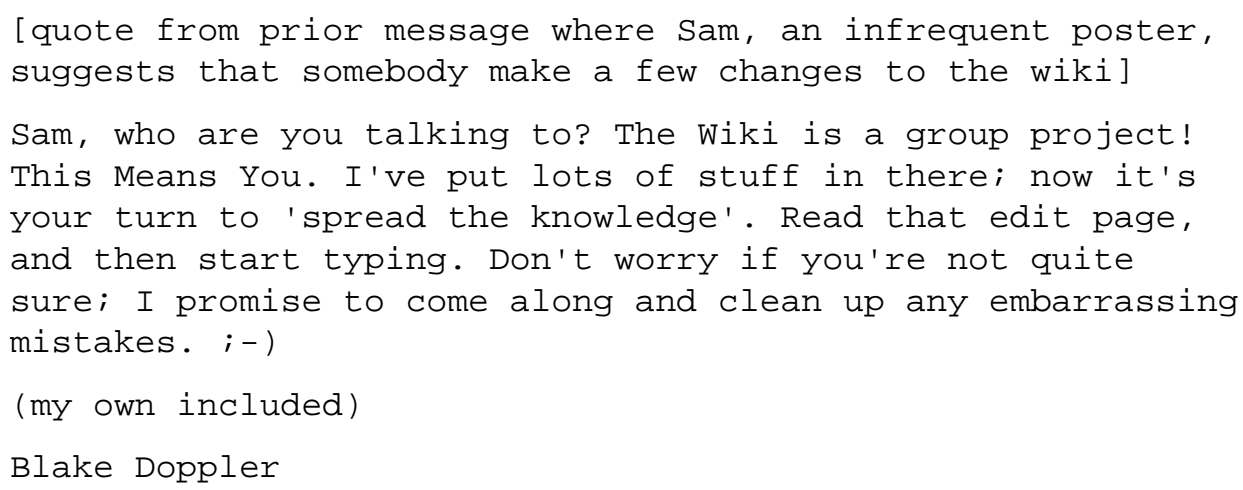

Because members recognize Blake and his social standing within the community, they are willing to accept his encouragement to make their own edits. The fact that he had made wiki edits before, including the creation of a few pages, likely helped his argument that it was someone else's "turn” to contribute. In this case, the suggested edits were made, although data does not exist to indicate who made them. This type of encouragement by core members and admins has been consistently provided to list members. For example, the original author of the ThreeColumnLayouts page mentioned that for a while he posted new layouts that people would email him, but more recently he has been "encouraging people to do more of their own work” by telling them “ “It's a wiki! Do it yourself.'” In a distributed environment with a lack of traditional incentives, the existing social structure helps members coordinate work in a largely acceptable 
manner. These examples show how social structures can help assign work, as well as provide a framework under which social norms about who can contribute can be developed and spread.

Not only was the email list community important in establishing the initial content, it has continued to play a key role. The creation of the IE7 page demonstrates the value of having community backing. When a new beta version of Internet Explorer 7 was released in late 2006, an administrator posted a message to the list announcing that he had created a new wiki page, IE7. He was concerned that discussions of the new release would swamp the email list and suggested that the page be used to collect information on how the browser handled CSS. Within days the community had posted CSS test results and bugs to the page, which was useful to many website designers and even the Microsoft IE7 development team. The timeliness and coordination of effort was largely a result of its “community backing.”

\section{Motivating Contributions}

As discussed in Chapter 2, one of the challenges of developing knowledge repositories is motivating individuals to contribute their knowledge. Although some individuals contribute to the css-d wiki for reasons unrelated to the email list (e.g., enjoyment; for personal use), the existence of the email list often provides additional motivation.

Interviews and evidence from the RecentChanges page suggest that most of the new wiki content is provided by core email list participants. These core members say that their major motivation for contributing is to lessen the amount of effort they will need to expend on answering frequently asked questions and dealing with Holy Wars. Indeed, this was the original motivation for the creation of the wiki in the first place as described in Chapter 5. The following quote from a core member who became an active wiki contributor is typical: 
After I spent a few months answering those questions, I realized the complimentary role of the wiki, that is, the same sort of question is arising over and over again.

Rather than having to draft a reply each time, it's much easier simply to refer to the relevant page on the wiki. That's when I started writing pages on those subjects on the wiki.

An administrator mentioned that he created the IE7 page so that the list wouldn't "drown in posts about IE7 Beta." Similarly, members that helped initially seed the wiki mentioned that they were motivated to create pages on Holy War topics so that they could offload the conversation when it became contentious.

One benefit of motivating core participants to contribute is that core participants are those with the most content expertise. This means that the pages addressing frequently asked topics or Holy War topics are written by individuals most likely to provide high quality content.

Another common reason for an individual to contribute to the wiki is because another list member has suggested they do so. The examples from the prior section describe how this is particularly effective when a prominent list member recommends that another member add something to the wiki. It also happens when typical members compliment another member on a particular post and encourage him to add it to the wiki, as in the following example:

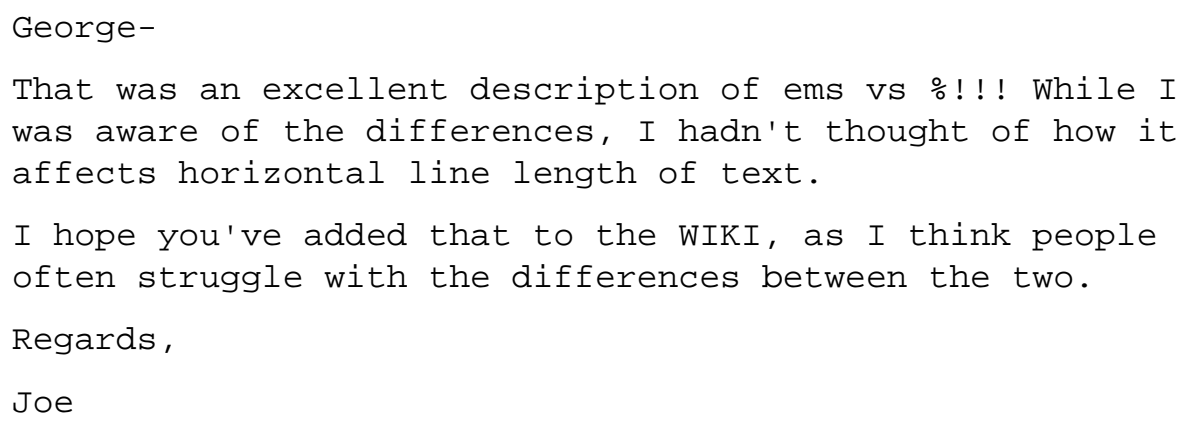

This message prompted George to create a page, announce it to the list, and suggest that others should "feel free to edit" since "It's a wiki. :-)". The validation of the original post can act as a strong motivator and indication that the content is, in fact, of 
more general interest than the original poster may have thought. This is strengthened by the fact that the praise and suggestion were offered in a public setting with thousands of people listening in. Even when people contribute to the wiki of their own volition (i.e., not at the suggestion of others), they can receive a virtual "round of applause" by announcing their edits to the list.

The email list also helps motivate wiki contributions because members who benefit from it want to "give back" to the community. As mentioned in Chapter 5, several individuals have helped remove spam from the email list as a way of saying "thank you” to the email list community which helped solve many of their problems. Many of these individuals are not content experts, and thus cannot contribute substantively to the email list conversation. However, removing spam, fixing spelling errors, and copying links from an email list message to a wiki page do not require content expertise.

Finally, the cost of contributing to the wiki is lowered substantially because content can be transferred from the email list rather than being created from scratch, as will be described in later sections.

Before leaving the topic of motivation it is important to recognize that the email list also introduces some motivational challenges. Several core members who were interviewed expressed frustration that other members did not contribute to the wiki more often. The following quote by an active email list participant is telling: "Unfortunately I didn’t invest much time in editing these [wiki] pages. I think people that get help from others on the css-d email list might update the wiki to give knowledge back to the community, to participate in the helping process.”

\section{Suggesting Topics}

The email list helps improve the wiki by suggesting relevant topics and providing peer reviewed content. Although these are related, we will address them each separately for analytic reasons. 
Many potential wiki topics are suggested by their frequent recurrence on the email list, whether they address frequently asked questions or frequently debated Holy War topics. The ConstantsInCss example above is illustrative. The nice thing about topics that are suggested in this way is that they are assured of being relevant to a large number of people. This is the power of the traditional FAQ. The questioners are the ones who drive what gets talked about, not a publisher or author standing apart from the crowd. Evidence from interviews and content analysis of wiki pages suggest that most wiki web design related pages were created as a result of their frequent recurrence on the list. In fact, all three members that were interviewed who played a role in initially seeding the wiki mentioned that the initial content was there to address frequently discussed (and debated) topics. Indeed, this was the rationale for the wiki in the first place. This purpose has largely been retained, as new topics are added due to their recurrence on the email list. The following quote from an email message sent at the end of a thread about a particular topic is typical:

I'll see if I can produce a page on the Wiki for this whole issue since it turns up from time to time.

Although most of the time experts are the ones who recognize repeat questions, sometimes novices inadvertently suggest content that should be added to the wiki (e.g., "I have looked on the wiki for vertical centering help, but don't see any.”)

Many similar messages indicate specific areas or issues that are not currently addressed by the wiki. They act as a signal to other members that the wiki should be updated to include information on the topic, or, if it is there, made more findable.

Importantly, this process leads to the creation of information that helps fill broader information gaps beyond those discussed within the community. The css-d community is only one patch within a broader information landscape including CSS books, tutorials, social networks, other web design email lists, and thousands of individual websites. Members at css-d send people to other CSS-related websites far 
more often than they send them to the wiki, because so many useful resources already exist. Indeed, many of the most frequently cited resources were created by list members who are CSS experts. However, in the few cases where good resources do not exist (or they are too hard to find), members use the wiki to fill the gap. This process assures that the wiki covers content with a high value added. This likely explains why several of the pages most often referred to in the email list discussion (e.g., ThreeColumnLayouts, BoxModelHack, CssHacks) are also popular outside of the email list (as evidenced by the search term analysis that identified them as pages most often referred to by search engines - see Table 5.2).

Another set of topics are suggested by the email list conversation because of their novelty, not necessarily their frequency. The email list has led to many novel approaches to working with CSS (e.g., new browser hacks or ways of achieving some design effect like rounded corners), especially in the early days of the list when CSS's interaction with browsers was not as well understood. The following quote from a long-time member discusses the role of the community in creating these novel approaches. It was offered in response to the question "Is there any content on the wiki that you don't think would've been there without the email list?”:

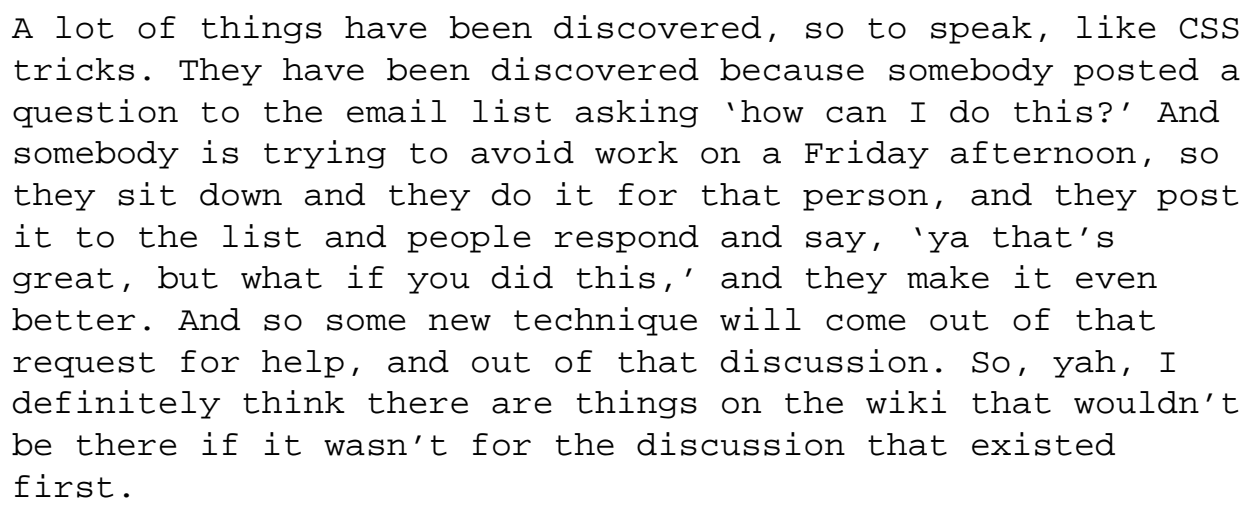

Another long-time member discussed the vision that early members had for the wiki as one of addressing frequently discussed topics, as well as preserving insights that were developed in the email list: 


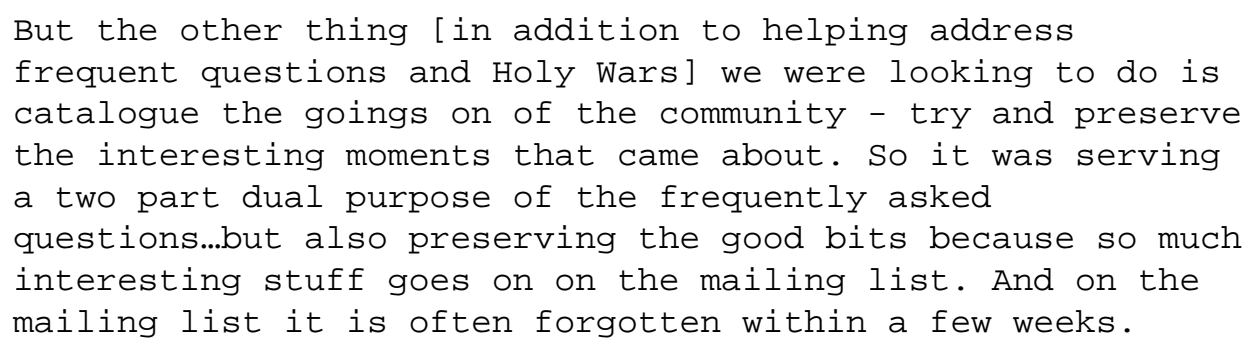

Other members have described making edits for similar reasons, namely, to capture some technique of lasting value that might otherwise be buried in the email list. As discussed in the previous section, several wiki pages were created as a result of suggestions from other members who thought the information was worth capturing in a more permanent way on the wiki. In these situations, the email list is helpful in suggesting the topic, as well as creating the environment where the information was made explicit in the first place.

\section{Providing and Reviewing Content}

The email list is also important in providing the actual content that populates pages in the wiki. Those contributing to the wiki don't need to start from scratch. They also benefit from the multiple perspectives and peer review that occurs in the email list, which helps them decide what the best content is to include in the wiki.

The ConstantsInCss example shown earlier demonstrated how an individual created a new page using content from an email list thread. Although the wiki page mentions that this is a question that some people come across, it does not explicitly talk about the source of the content. This is typical. Although a few wiki pages link to email archive threads (3\% of all wiki pages), and a few more mention that content was adopted or directly copied from email threads, most of the time the provenance of the content was not identified. However, all interviewees, except one wiki contributor who is not a member of the email list, mentioned the email list as a primary source for many of their 
edits. Furthermore, nearly all of the 66 messages discussing prior edits (see Table 6.1) make it clear that the edit was related to content that was discussed in the email list.

Not only is the current conversation an important source of content, the public archive is also important. Several interviewees mentioned using the archive to help gather information on a particular topic before posting it to the wiki. One wiki page begins with the following quote, which references the author's "long and hard" archive search for the material:

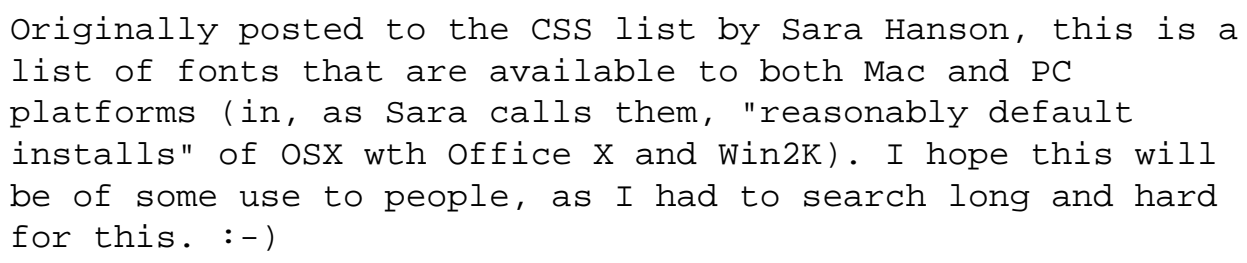

Another wiki contributor described how he found a helpful technique on a subject he was researching in the email archives and "posted it to the wiki because I was frustrated that I didn't find it in the wiki, so I went and found the answer and then I organized it and then put it into the wiki.”

This last quote hints at the additional work performed when transferring information from the email list to the wiki (discussed more fully in Chapter 5). The work of decontextualizing, summarizing, organizing, and aggregating information often requires content knowledge. We saw in the ConstantsInCss example how one member was hesitant to edit the text in a particular section because they feared introducing some problem. Other similar cases exist. Thus, members without expertise on a given topic may sacrifice coherence for accuracy. At the other extreme, editors of Article pages are often the most knowledgeable and most able to summarize threads into a coherent and reusable form.

In addition to reducing the effort required to contribute to the wiki, transferring content from the email list helps improve the diversity of the wiki content. One of the strengths of the wiki (discussed in Chapter 5) is its ability to aggregate various techniques 
and information. This is partly a result of its close association with the email list. Even when a single person contributes content to a wiki page, they are often including comments from a number of other individuals (e.g., the ConstantsInCss example). One active email list member who does not edit the wiki mentioned that he contributes to the wiki by providing information to others who put it on the wiki. This suggests that some members recognize their email list contributions as potential wiki contributions.

The quality of the wiki content is also improved because it comes out of the email list. This happens for a few reasons. First, as discussed in the prior section, some content is added because it was identified by other members as particularly insightful. This "biased" selection process assures that the wiki content will be of higher quality than an “average” email list message. Second, the conversation is occasionally used to validate information being added to the wiki. The following quote from an active email list and wiki participant describes this process:

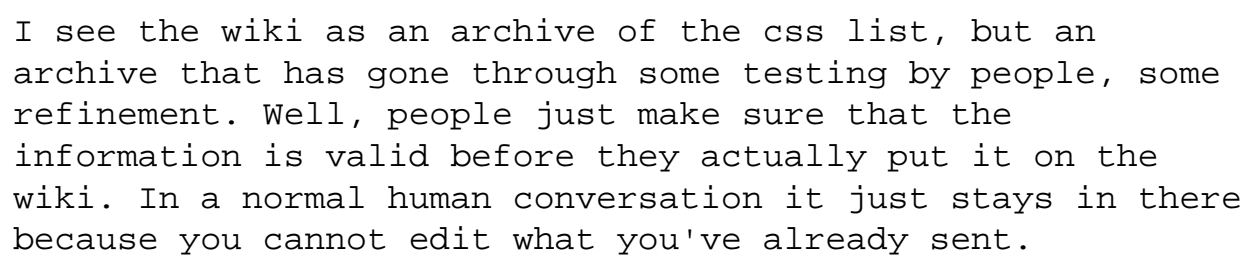

Many of the 152 messages that discuss future wiki edits (see Table 6.1) explicitly ask for other members to review a contribution either before or after transferring it to the wiki. The following quotes taken from a few of these messages are indicative:

$i$ think $i$ will add that to the "ShowCase" wiki page if no one objects.

I'd add it to the WIKI, but do we want it as a commercial site, or should we add a non-profit heading?

After being surprised that it wasn't there, I added a quick article about Dropcaps to the wiki. Contributed wisdom and useful links are welcome.

once we all agree, can someone post it on the css-discuss wiki? ;)

Should this be renamed "CustomCorners"? 
Many of these requests lead to edits or comments about the appropriateness of certain edits. This may be as simple as a validation that something is a good idea, or it may include long discussions about a particular topic and discussions of specific wording to be added.

Even when individuals do not explicitly solicit the community, much of the content is still reviewed as part of the regular email list activities. The ConstantsInCss example demonstrated how a particular technique was rejected by another member as not being a best practice. The result was that it was not added to the wiki.

\section{Identifying Problems with Wiki Content}

Even with the selection and review process that information goes through before getting transferred to the wiki, the content is imperfect. Problems occur in various forms, such as confusing or ambiguous language, grammatical and spelling errors, redundancies, confusing content organization, broken links, outdated information, missing content, and incorrect information (e.g., errors in a CSS code snippet). Because much of the wiki content is reused in the email list, many of these problems are identified and brought to the attention of individuals who have the expertise to fix them. In this way, the wiki content that is reused in the email list conversation is maintained over time. This maintenance occurs in several ways as described in this section.

Many problems with wiki content are initially identified by novices who search the wiki before asking a question to the list. Their lack of content expertise makes them particularly adept at recognizing certain types of problems such as ambiguously worded statements and the excessive use of jargon. These problems are brought to the attention of the email list either explicitly or implicitly when the novice asks for help in resolving their concern. This acts as a signal to the list experts that there is a problem with a page, or perhaps an opportunity for improvement. The following message posted by a novice is illustrative: 


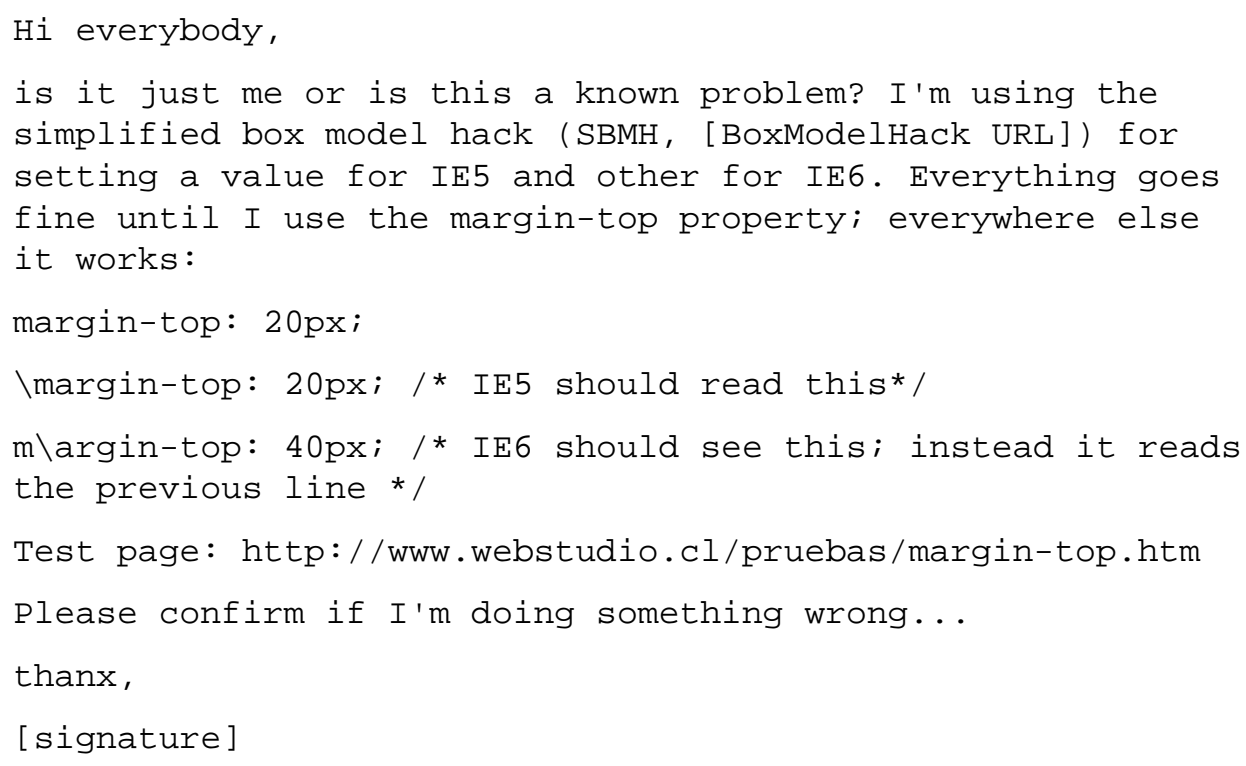

List members immediately replied to this message indicating that the author had misinterpreted the paragraph in the wiki. After a bit of discussion among core members, one of them posted a message indicating that he had changed the page in order to "avoid confusion on this in the future.” This example shows how the email list made visible the reader's misinterpretation of the wiki content. In other cases, wiki readers explicitly recognize problems with wiki content and verify their hunch with the list, as in the message that began "I think there's a mistake on this otherwise excellent resource, penned (I believe) by Danny Gilles.”

Problems are also identified and fixed because experts use wiki pages to help answer email list questions. As previously discussed, the primary reason for referencing the wiki is to help educate another member (see Table 6.1), typically as part of an answer to a question. This is nearly always done by linking directly to a wiki page. Interviews revealed that experts often visit wiki pages before linking to them. This is done for two reasons. First, they often visit a wiki page in order to copy and paste the URL into the email message (since they are not easy to remember). Second, they sometimes check to see if the page actually addresses the particular question posed in the email list. These visits by experts have led to edits that help maintain and improve wiki pages. Sometimes 
experts simply see spam that needs to be removed, while other times they notice information they "thought was incorrect or poorly organized" and "stop and clean it up." One member mentioned that he "wouldn’t ever send someone to a page without first checking through it that the content hadn't changed.” One nice outcome of this process is that experts visit sites that they might not otherwise visit, not for themselves, but so they can use them to help answer other member's questions. This assures that even topics of interest primarily to novices still get occasionally reviewed by experts.

Data suggest that wiki pages that are referenced in the conversation are edited more often than those that are not. When excluding the SandBox and FrontPage (because of their unique purposes), the average number of edits of all referenced wiki pages is 24 (median 19); for all wiki pages, the mean number of edits is 9 (median 4). Likewise, the average size of all referenced wiki pages (3.7 KB; Median 2.8 KB) is also larger than the average size of all wiki pages (1.4 KB; Median 0.5 KB). This is not surprising since most edits of a page add content to it.

Data also suggest that pages that are referenced more often in the conversation are more likely to be edited than those that are referenced infrequently. For example, among the 112 referenced wiki pages, there is a positive relationship between the number of times that pages were referenced and the number of times that pages were edited (0.65). A multiple regression was performed to assure that this correlation was not attributable to other variables including the age of a page and the popularity of the page's topic (estimated by the number of inlinks to the page from websites other than the wiki or archives). ${ }^{5}$ The results show that the positive correlation of references and edits is

${ }^{5}$ A linear OLS regression was performed after taking the natural log of all variables to account for their skewed distribution. The resulting regression equation was: $\operatorname{Ln}($ Edits $)=+0.18 * \operatorname{Ln}($ Email List References $)+0.24 * \operatorname{Ln}($ Inlinks $)+1.02 * \operatorname{Ln}($ Days Since Creation) -5.20 . The $\mathrm{R}^{2}$ was .56. All variables were significant at the .01 level. The number of days since the page's creation is not an exact number, but is based on the 
significant $(p=0.009)$ even when accounting for the other variables. The coefficient suggests that a $1 \%$ increase in the number of references corresponds to an $0.18 \%$ increase in the number of edits on a page (doubling the number of references would lead to an $18 \%$ increase in edits). The other variables were also significant at the .01 level and in the expected direction: a $1 \%$ increase in the number of inlinks corresponds to a $0.24 \%$ increase in page edits, and a 1\% increase in the number of days the page has been around corresponds with a $1.02 \%$ increase in the number of edits.

The positive relationship between references and edits is suggestive that the maintenance cycle is occurring and that it works better for pages that are referenced often than for those that are infrequently referenced. The causal link likely goes in both directions. Individuals discuss prior wiki page edits in $4 \%$ of the messages that reference the wiki (see Table 6.1). They also discuss future edits (e.g., recommend that someone make an edit to a page) in $10 \%$ of all messages (see Table 6.1).

Although the reuse of wiki content helps improve its quality, it is important to recognize the limitations of this process. The major limitation is that not all wiki content is reused. The majority of pages are rarely, if ever, referenced in the email list discussion. Furthermore, because the css-d wiki does not have well-established roles related to cleaning up the wiki (as other wikis such as Wikipedia do), pages that are not part of the reuse cycle are prone to become outdated and not improve. Indeed, nearly all members that mentioned problems with the wiki identified the existence of obsolete or dated information as the major one (see Chapter 5 for more discussion on this).

\section{How the Wiki Influences the Email List}

snapshots of the wiki. Inlinks for each page were taken from Yahoo! Search Site Explorer on August 18, 2007 and exclude inlinks from the wiki and archive's home domain. 
In this section I focus on how the wiki influences the email list. Specifically, I claim that the wiki helps the email list by:

- making question asking and answering easier due to reuse

- providing a place to offload problematic discussion

- attracting new members and helping them integrate into the community

- retaining long-time members

- motivating and focusing contributions

\section{Making Question Asking and Answering Easier Due to Reuse}

The most obvious way that the wiki influences the email list is by facilitating reuse. Content that is captured on the wiki can be referred to in the email list without having to recreate it. Furthermore, because the wiki is a shared community resource, members can assume that other members know about it and are familiar with it. Although reuse of wiki content in the email list discussion can influence all email list activities (see Chapter 4 for a discussion of them), this section focuses on its importance in aiding question asking and answering, the primary activities occurring on the email list.

\section{Question Asking}

Like an FAQ document, many of the wiki pages (e.g., BoxModelHack, RoundedCorners, CenteringBlockElement, FontSize) are written primarily by experts so that they can use them to answer novice questions in the future. The result is that the wiki includes topics that are suggested by novices, along with summary write-ups that are intended for novices. These pages often help members ask questions more effectively and efficiently. The following quotes from different messages are typical:

- We'd like to implement the @import hack (as described at [ImportHack wiki URL]) to provide Netscape 4.x users with... The trouble is that... 
- I've browsed through the excellent compilation of cSS based "floated" designs found in the wiki of this mailing list, and found several I can use as a basis. However, I've run into some challenges/problems concerning the header.

These messages show how members asking questions can use the wiki as shorthand for explaining a concept. Instead of describing the entire “@import” hack, the member simply links to the wiki page that describes it. Instead of describing all of the "floated designs" they have considered, the author can point to a page with all of them and explain that none of them work with his particular problem. This reduces the amount of work of the author, and since following wiki URLs is fairly common and easy, it does not significantly increase the reader's burden.

The practice of referencing URLs to help ask questions is not only restricted to the wiki. In fact, members most often reference URLs from other sites. However, referencing the wiki differs because it is a community resource and it can be assumed that others are familiar with it. Thus, members can raise questions such as the following with confidence that other members will be familiar with the layouts, techniques, and topics referenced:

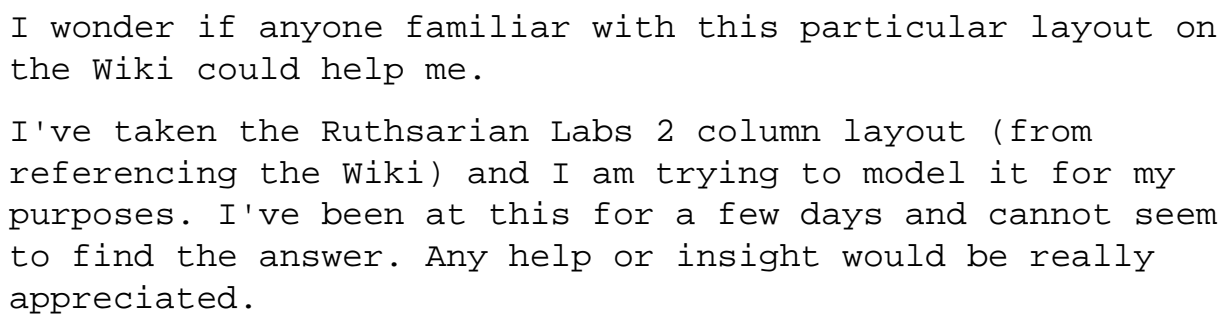

This technique of referencing the wiki can also help eliminate duplicate effort.

When answering a question it is difficult to know what the question asker already knows and has already considered. This problem is particularly difficult, because most help is provided to strangers. One technique that question askers have used is to reference the wiki to point out techniques that have been tried, but not been successful, as the following examples do: 
- Unfortunately, it doesn't work in IE for Windows, and the various hacks I've come across (almost all of which are listed on [ScriptHacks wiki URL]) don't work.

- I have been searching here: [ThreeColumnLayouts URL] and a few other places and have been unsuccessful [in finding a layout that fits the following criteria].

- I have looked at the technique used in the cSSDiscuss WIKI, but I'm experimenting with another method.

During the 28 month period, a total of 248 messages referenced the wiki in order to help ask a question or justify a post (see Table 6.1 ). This represents $16 \%$ of all wiki emails. Analysis of the 248 messages suggests that members use the wiki to help know what to ask and how to ask questions, refine their questions, ask questions more efficiently (by referencing a particular technique described on the wiki), and justify that they have done their homework (and thus deserver an answer). During the same time period, only 60 messages referenced the email archive (see Table 6.1). Messages that referenced the wiki and email list were envoked for similar reasons. However, they differed in that individuals that referenced the wiki were more likely to mention specific techniques that were borrowed from the resource and to link to specific wiki pages. Individuals that referenced the email archive were more likely to simply justify their question by stating that they had searched the archives without finding the answer. This analysis suggests that the wiki is more frequently used than the archive to help ask questions and more useful when questioners want to explain the use of a particular technique.

One newcomer described how he regularly searched the wiki before posting in order to "get more know-how to ask a question," especially since it was "going to a lot of people - CSS experts.”

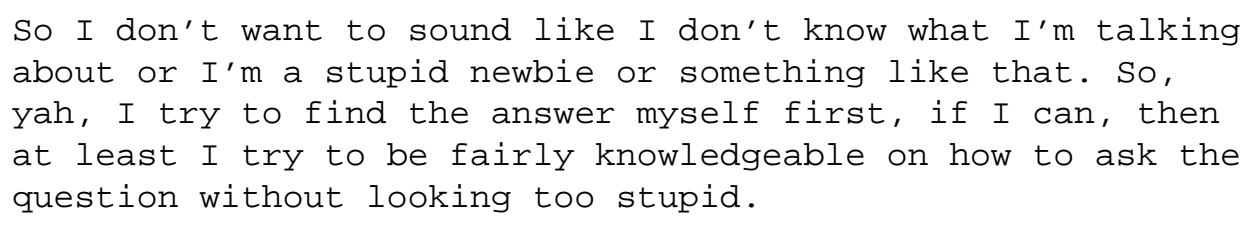


This ability to become knowledgeable before acting enables peripheral participation (Lave \& Wenger, 1991), which is an important factor in helping people move from a peripheral to a central role.

In addition, members can use a technique from the wiki (and post questions about it) with confidence that it is an accepted technique recognized by the community.

Although this can be done with techniques from the email archive, those on the wiki have increased credibility because of its selective nature. They are also beneficial because they are summarized and organized in a single place. The following quote explains this benefit:

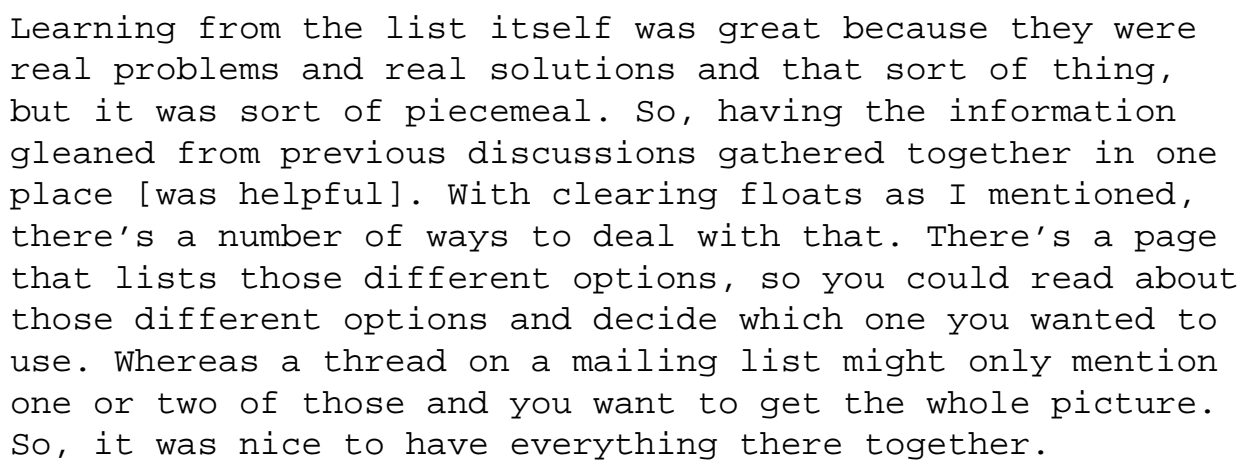

\section{Question Answering}

The wiki also helps efficiently answer questions, especially frequently asked questions, when they come up on the list. This is especially important at css-d because of their policy that there are "no stupid questions.” As discussed in Chapter 4, the list policies make it clear that "simply posting a URL as an 'answer' is also discouraged." Members are told that they should back up that URL with "a little explanation of what the reference is about, why you posted it, and some keys to understanding the resource you're referencing.” Most members follow this counsel when referencing the wiki, so that instead of serving as a substitute for answers it is used primarily as an addendum to them.

The usefulness of the wiki in helping to answer questions is evidenced by its frequent use for that reason as compared to the archives use for that reason. The most 
common reason for referencing the wiki (61\% of all wiki references) is to help answer questions and educate others (see Table 6.1). The email archive is used for a similar purpose (51\% of the time), but is only referenced 1/6th as often as the wiki (158 times compared to 917 in the same time period) (see Table 6.1). Both resources are used for this purpose so often because answering questions is so common an activity. However, the fact that the wiki is referenced 6 times more often than the archives suggests that it more effectively supports the reuse of information than the archive when answering questions.

The following example is typical of how questions are answered by referencing the wiki:

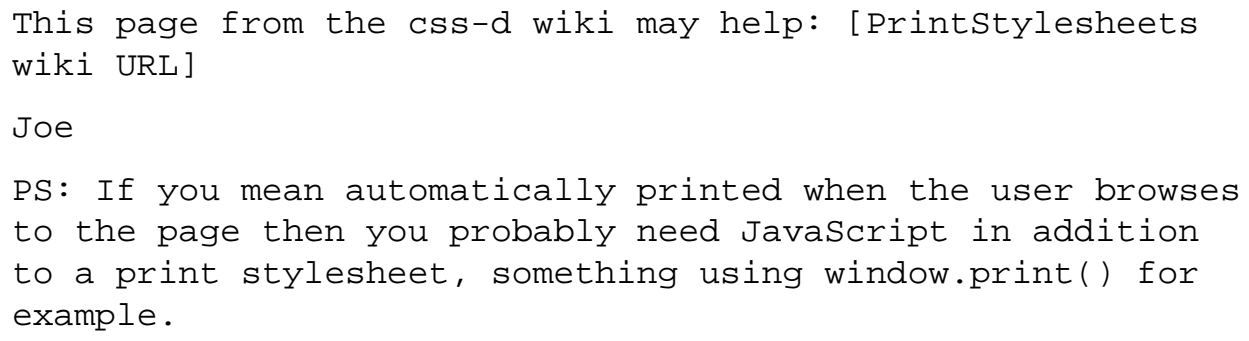

This short reply answered a brief question that was somewhat vaguely worded.

The reference to the PrintStylesheets wiki page is intended to give the person some ideas about how to approach the problem. As is typically the case, Joe links to a specific wiki page (as opposed to providing a general link to the wiki). Referencing a specific wiki page occurs $86 \%$ of the time when the wiki is referenced in order answer a question or educate. As is typical, more than just the link was provided. The wiki is particularly helpful in addressing these difficult-to-answer questions (either because they are poorly asked, too general, or difficult). One admin described how he used the wiki to help answer the questions that were left unanswered by others:

I try to look for the unanswered things and go answer them or I'll point them to where they'll find more information. So that's one way the wiki is really handy. Someone on the list may not have a direct answer or may not have wanted to take the time to answer someone a particular question, so 
if you can point people to a page on the wiki, at least it gets them off in another direction.

In other situations, especially when the question is more specific and clearly worded, the answer is longer and includes a link to the wiki as a source of additional information on the subject. The following is typical:

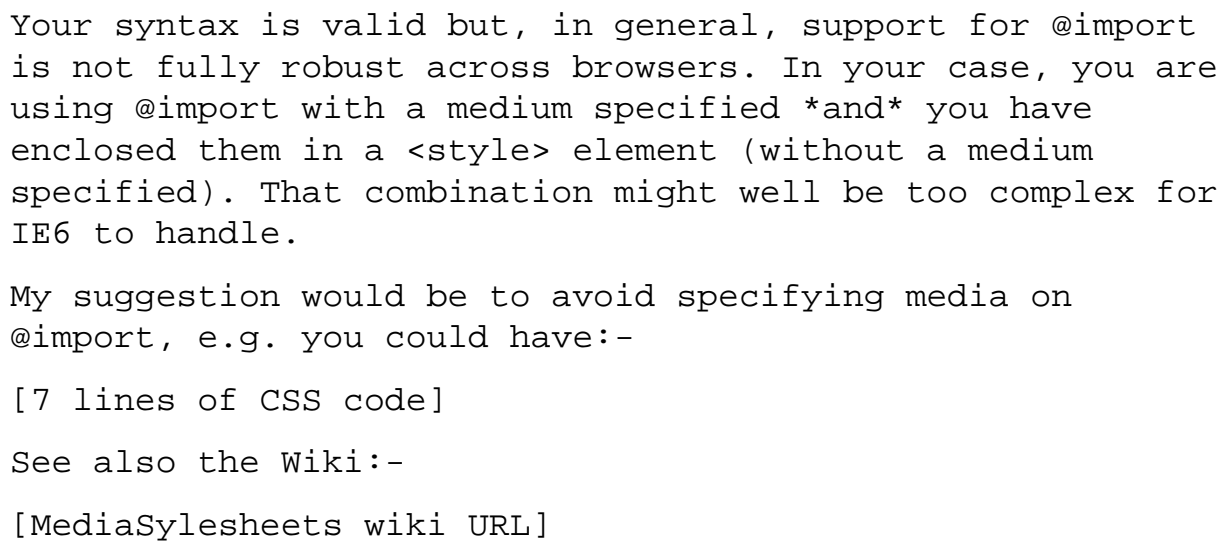

In this example a specific solution was proposed, followed by a link to a wiki page as an addendum. This practice helps educate the reader more generally, making it more likely that they will understand why the suggested solution will work. In fact, wiki pages are often referenced in order to explain the "why" behind things, since they often discuss topics in more depth than the average email post. The following message is telling:

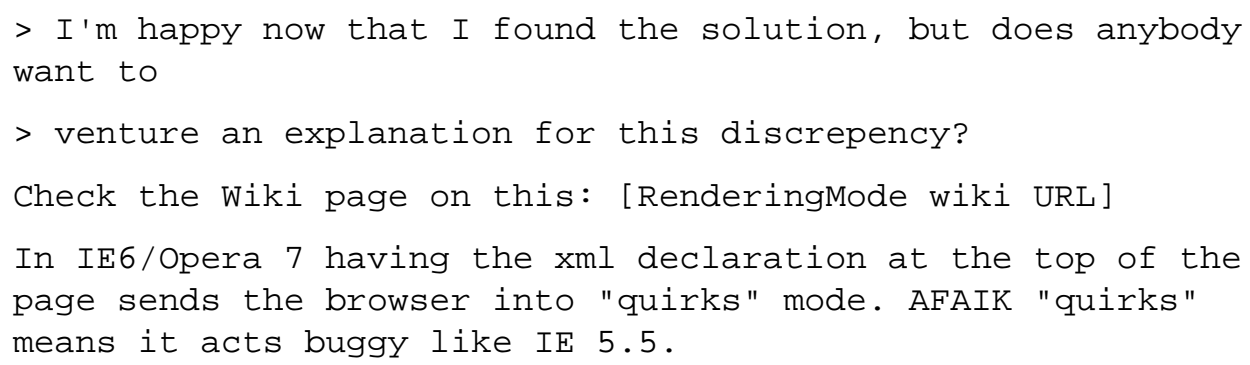

Although there is a brief explanation in the email, a more complete description is provided on the wiki page. As discussed in Chapter 5, many wiki pages are decontextualized summaries, and/or aggregate information from disparate places. Thus, 
when members refer question askers to a wiki page, it is likely that the content there will be more in-depth and comprehensive than they would receive in the email list by itself.

Finally, the wiki serves as an external memory to help question answerers find answers. Even the most expert members mentioned using the wiki for this purpose. The following quote by a CSS expert was provided in response to an interview question asking how he uses the wiki currently (as opposed to how he used it when he first joined the list):

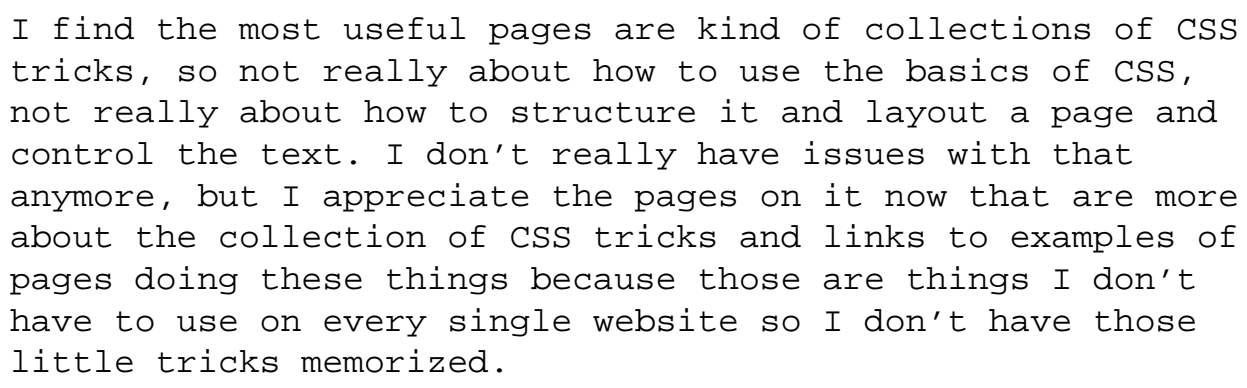

This member later mentioned how he uses the wiki as a collection of bookmarks because he likes "having everything together in one place.” This example demonstrates how even experts rely on external memory resources.

In summary, referencing the wiki helps question answerers to easily include extra information that is often more comprehensive and detailed, as well as refresh their memory when answering others questions. This lowers the cost of answering questions, as well as increases the efficacy of the answer.

\section{Providing a Place to Offload Problematic Discussion}

This section describes how the wiki has been useful in offloading two types of problematic discussion: off-topic discussions and Holy Wars. Table 6.1 shows that 74 cases were found where the wiki was used to help offload the discussion because it was off-topic or considered a Holy War. Interview data suggest that these are only a small fraction of the total times the wiki was referenced to help offload the discussion, since the 
majority of times these messages were sent directly to individuals rather than to the list as a whole. In contrast, only 3 messages during the same 28 month time period were identified that referenced the email list archives for a similar reason. The following discussion helps explain why the wiki has been particularly useful in managing these issues.

\section{Avoiding Off-Topic Discussion}

As discussed in Chapter 4, css-d (like many online support communities) must cope with the challenge of keeping the discussion on-topic, while still meeting the immediate needs of its members. The wiki has been used to help successfully negotiate this boundary by providing a new method for dealing with off-topic content. To understand how this plays out, a typical off-topic example is presented below. The example began when a "newbie” asked for recommendations of Content Management Systems (CMS software) that were CSS compliant. Within a few hours he had received 7 replies from community members, which suggests that this topic was of interest to several members. It was, however, technically not on-topic. The final message in the thread was sent by a list admin who shut down the thread as shown below:

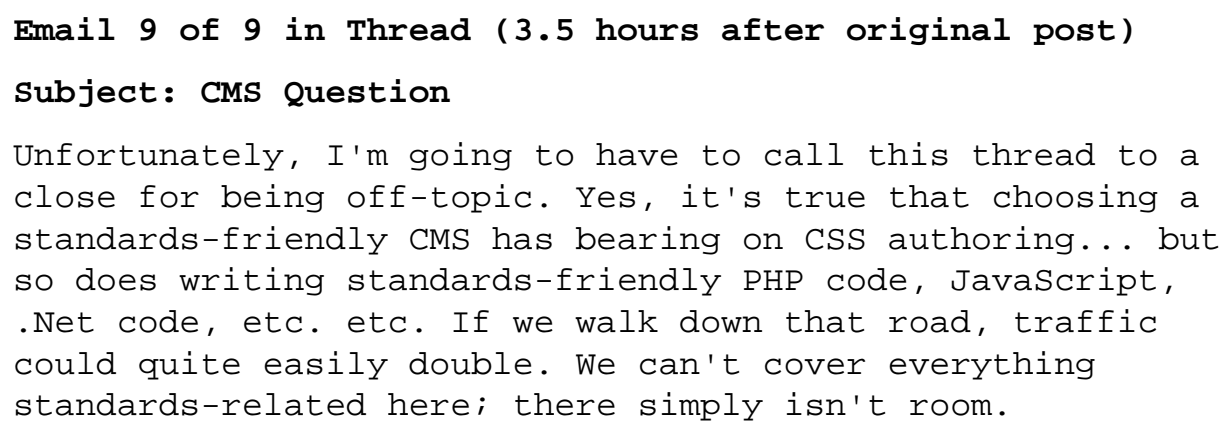

A great place to ask your question would be Webdesign-L. There's also evolt's thelist, and I'm sure there must be other venues where

CMS discussions are on topic. For a short list of forums, see [URL for OffTopic wiki page]. On the topic of CMS software, see the page others in the thread have brought up: [URL for CssFriendlyCms wiki page]. 
Thanks, and sorry to have to end the thread.

There are several things to note from this example. First, the admin is more courteous than he is in some other comparable posts, most likely because the offender introduced himself as a "newbie.” This is evidenced by the apology for ending the thread, the pointers on where to go, and the explanation justifying the list policy. Second, the admin references the wiki's OffTopic page, which is an annotated list of links to related discussion groups. It also includes a brief description of what is on- and off-topic at css-d. Finally, the admin links to another wiki page (CssFriendlyCms) that discusses the questioner's very topic and had already been pointed out by other list members. The page includes details on how well various CMS products interact with CSS.

In this example (and others like it), the wiki complements the email list discussion. While the email list is a push technology that is broadcast to all members of the list, the wiki is a pull technology that requires members to actively seek it out. Members at css-d have used this combination to their advantage. Wiki content is allowed (and even encouraged) to cover topics that are "on the margins of on-topicness" but are technically off-topic (e.g., CssFriendlyCms, Javascript Hacks). These pages do not force themselves into anyone's email box; hence, they bother people less. However, members that do care about the topic can use the less invasive wiki technology to educate one another and collaborate if necessary. Likewise, when newcomers post off-topic messages, it is possible to send them to a useful, community-created page rather than blowing them off altogether or taking the time to answer their question in a private message.

The existence of the OffTopic wiki page is also helpful in reducing the admins' workload, while being friendly to newcomers. One admin put it this way: "if you just tell them that [a post is] off-topic, they will often email back and say, 'well, where else can I learn about this?' And so then you gotta point them to Webdesign-L's list or Evolt's thelist or a bunch of other common mailing lists, so instead it's just all there. So we don't have to type the same email out to them several times.” The OffTopic page also helps 
non-admins keep the discussion on-topic by lending credibility. In fact, one-third of the references in emails to the OffTopic page were posted by non-admins.

Although the examples discussed so far occur after-the-fact, the wiki has also been used to preemptively offload discussion on certain hot topics. For example, in order to reduce some of the list traffic about the Internet Explorer 7 beta release, one admin created an IE7 wiki page and encouraged members to use it as a place to collect and share testing results. One of the primary reasons this approach was taken was to "avoid having the list drowned by IE7 testing results and related traffic.”

\section{Avoiding Holy Wars}

Another way the wiki helps the email list is by helping the community shut down or avoid Holy Wars, or un-resolvable debates with little practical value (see Chapter 4 for details). Admins try to avoid Holy Wars because, like off-topic discussion, Holy Wars are generally only of interest to a few, but demand the attention of many. Furthermore, when they lead to flaming, relationships can be damaged, members may become more hesitant to ask questions of their own, and members that are sensitive to conflict may even leave the community. The following message was sent by an administrator after a font sizing Holy War broke out, where members continued a fruitless debate about whether or not users should be expected to change their browser's default text size settings. As often happens in these cases the debate was also becoming personal as evidenced by members' use of strong language, numerous exclamation points and all-caps statements for emphasis, and comments directed at one another rather than the issue at hand.

Email 16 of 17 in Thread (23 hours after original message)

If there is one thing this list will never, ever make possible, it's a final resolution to the Font Sizing Holy War. This is why we established a Wiki page that gives people a good jumping off point for reading up on various perspectives: [URL for FontSize].

The next time the war breaks out, let's just refer each other to that page and move on to discussions of a less 


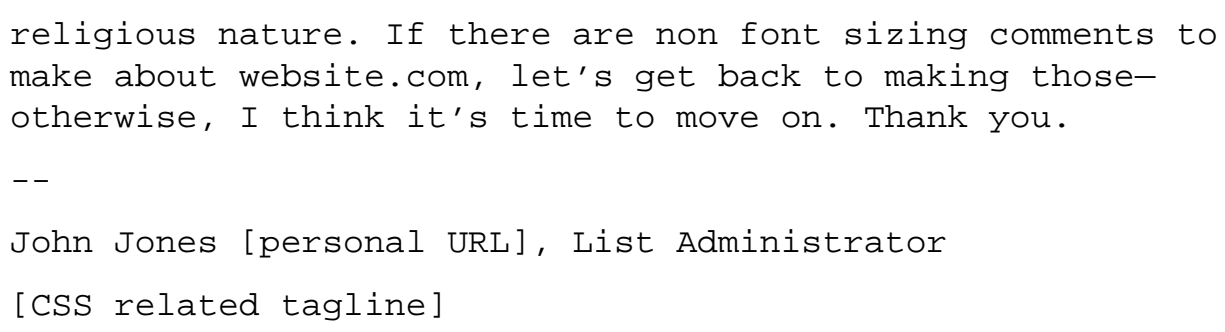

In this example, a list administrator ended the holy war by pointing list members to the FontSize wiki page (a typical example of a Debate wiki page described in Chapter 5). That page and related pages describe how to use the various font sizing techniques and summarize their potential benefits and drawbacks. The admin's suggestion to reference the wiki page to shut down the font size Holy War was followed many times in the future, as were linking to other Debate pages to shut down Holy Wars on other topics.

Diverting holy wars by pointing to an FAQ on the topic is not new. However, the differences in who can edit the page and the format of the page are significant. Because the wiki is editable by those who are being diverted, they have the option of contributing their knowledge rather than being completely shut down. However, their contributions are not forced upon anyone since the wiki is a pull technology. In fact, some members have recognized the usefulness of this combination and diverted Holy Wars by combining personal email with proposed wiki edits as in the following example taken from an email list message on a potentially contentious topic:

Since I * know$^{*}$ this could turn into an opinion bashing spam
hate-war argument flame arena....... Please do NOT respond
to the list. Instead, please respond to me directly and, as
I said, I will be happy to include my findings in the Wiki.

The format of the wiki and the shared understanding of its purpose help make it effective at shutting down Holy Wars and not simply moving them to the wiki. One list administrator described his approach this way,

What I stressed was to present [the contentious topic] with as little heat as possible on the wiki. If there were two sides to an issue they should be presented fairly and sort of neutrally. Document the pros and cons. And if somebody couldn't think of any cons for their preferred method, they 
could write up the pros and someone else could think up the cons and can add them.

As described in Chapter 5, several of the Debate pages have done just that. The structured format and established neutral tone of the wiki has encouraged the useful distillation of even the most problematic discussions. Indeed, a previous holy war participant, when interviewed, stated that she did not feel put off by this approach because she felt like she could still voice her opinion on the wiki. The result is that holy war participants are encouraged to turn their "weapons into plowshares,” by converting their arguments into a useful information product instead of using it to endlessly debate or belittle others.

\title{
Attracting New Members and Helping Them Integrate into the Community
}

\author{
Attracting New Members
}

Another way that the wiki has helped the email list is by helping attract new members. As discussed in earlier chapters, the css-d community has several possible online entry points including the wiki, a static community website, the public email archives, and the founder's website. Google searches for “css discuss” and “css discussion list” made on January 27, 2007 brought up the wiki’s home page as the first result, suggesting its importance in relation to other pages. However, server logs suggest that the email archive receives 3 times more unique visitors than the wiki. Despite this fact, the wiki plays a significant and unique role in the promotion of css-d.

Many members are initially introduced to the community through the wiki. In January 2007, there were approximately 10 times more unique visitors to the wiki than members subscribed to the list. As discussed in Chapter 5, most connections to the wiki site are from search engines (44\%) or websites not affiliated with css-d (18\%), avenues likely to attract individuals new to the community. In comparison, 33.7\% of connections were direct access (i.e., from bookmarks or following links in email clients) and 4\% were 
from other css-d websites such as the archives, avenues likely to be used by existing members. Analysis of the search terms suggests that newcomers find the wiki by using search phrases on specific CSS related topics like "three column layout," "css hacks," and "css font size" and are initially taken to the specific wiki pages on these sub-topics (see Table 5.2). In fact, only 7 (0.7\%) of the 1,000 most frequently used searches were related to the name of the community (e.g., "css-discuss”, “css wiki”, “css-d” and other variations).

The technical structure of the wiki and its use by the community have contributed to its ability to reach new members. The persistence of wiki page URLs allows individuals to link to a page with confidence that it will be there in the future. Although it is technically possible to change the URL of a wiki page, it has rarely been done. Because content on a wiki page can be updated, it is more likely that pages will stay up-to-date than links to unchangeable, archived email messages. Thus, the combination of persistent URLs and changeable content makes it likely that individuals will link to specific wiki pages. In contrast, the email archives have a persistent URL for each message but are not updatable. This is likely why so few people (2.6\% of all visitors in January 2007) come to the archives by following non-search engine external links. In fact, almost 3 times more people follow external links to the wiki than to the public archives.

An analysis of inbound links to the wiki and archive was performed on August 16, 2007 using the Yahoo Search Site Explorer. All inbound links from sites in the Yahoo directory were included except for those originating from the wiki and email archive. A total of 545 inbound links pointed to the email archive main page and none pointed to specific email list message pages. In contrast, there were 35,629 inbound links pointing to the wiki. Of these, 16,247 (46\%) linked to specific pages other than the FrontPage, RecentChanges, and rss feeds. For example, over 4,000 links were directed at the ThreeColumnLayouts page, and over 1,000 were directed at the BoxModelHack and IE7 pages respectively. This supports the argument made above, that the wiki's structure 
encourages others to link to specific wiki pages, in contrast to linking to specific archive messages.

Linking to wiki pages from external sites increases the chance that others will stumble upon the wiki (and thus the community) and increases the likelihood that individual wiki pages will show up high in search engine results (i.e., it increases the wiki’s Page Rank) (Brin \& Page, 1998). Furthermore, this process makes it more likely that newcomers are exposed to the most popular (and presumably most useful) wiki pages first. For example, the IE7 page was created to document how well the new version of Internet Explorer supports CSS. Dozens of community experts posted CSS test results and bugs to the page. Because of its usefulness and timeliness, 1,133 external sites linked to the IE7 page. The result was that many individuals (including the Microsoft IE 7 development team) were introduced to the community through the page which showcased the expertise of its members.

\section{Integrating New Members}

Not only does the wiki attract people to the community, it also helps newcomers integrate into the community. The most notable way this occurs is through helping them ask informed questions as described earlier in this chapter. In addition, members can refer to a technique found in the wiki (as they often do) with confidence that the technique is an acceptable one (or at least recognized one) by the community. This can be particularly important when discussing questions related to contentious issues such as font sizing.

Because the wiki content is heavily influenced by the email list discussion, it represents the core values, knowledge, and interests of the community. Potential members can browse through the wiki pages indexed on the front page and get a quick overview of topics most salient to the community. Although exploring the public email list archive can also provide this information, its lack of organization, repetition, and 
length (of over 85,000 messages) make it hard to quickly digest. The following excerpt from a new member's first post suggests the value of the wiki for newcomers:

The penny dropped about why CSS was a good idea only last Friday, but since then, thanks to running across cssdiscuss Wiki site early on, I am learning quickly. I am bowled over by this excellent site - valuable information, clear presentation, helpful people and I love the idea of Wiki.... Thank you all for the time you have already taken to share your hard won knowledge with me.

By reading the wiki this member was able to "learn quickly" (i.e., increase their knowledge of CSS) because he found the wiki early on and because it includes such valuable and clearly presented information. This will help him know enough to ask informed questions, as he did in the rest of the email. Notice also that the author was able to ascertain from reading the wiki that the members were "helpful people" willing to share their "hard won knowledge." As described in Chapter 4, these are some of the most central characteristics of the community (i.e., being helpful to newcomers and capturing hard won knowledge).

The wiki also includes pages describing list policies (e.g., PostingGuidelines), as well as helpful suggestions for newcomers (e.g., a page explaining how to reduce excessive quoting when using Gmail). These pages help newcomers learn how to follow the established norms of the community. Although some of these are available elsewhere (e.g., the css-d website and list chaperone's personal webpage), the wiki includes some content that is not available elsewhere (e.g., SearchCssDiscussList, GmailAndCssDiscuss, OffTopic). For example, the page helping newcomers use Gmail without over-quoting was created, at the suggestion of an admin, by a non-admin core member. Because the wiki is editable by any community member (unlike the css-d main site that is only accessible to the admins), they are able to contribute to these policyrelated issues in a permanent place.

\section{Retaining Long-Time Members}


The wiki has helped the email list retain core email list participants as well. While some attrition naturally occurs in any technical support community (as people change jobs or lose interest in the subject-matter), other times it is caused by the tension between supporting both newcomers and old-timers. Newcomers often have "newbie” questions that wear on the patience of old-timers. Conversely, old-timers often want to discuss more advanced topics or continue conversations without having to bring everyone up to speed. At css-d, where the primary focus has always been on supporting newcomers, there is a stronger possibility that old-timers will not stick around. Although some core members no longer actively participate in the list, there are many who have stuck around for extended periods of time. The wiki and the social practices around its use have played an important role in retaining these old-timers while still meeting the needs of the newcomers as described below.

As discussed in Chapter 4, newcomers are encouraged to look for an answer to their questions in the wiki and email list archives before posting to the list. However, as in other community question answering systems, this practice is not strictly enforced or adhered to (see Ackerman \& Palen 1996), leading some members to complain that newcomers don't use the wiki and archive enough. Even so, several long-time members mentioned that they believe the wiki has led to fewer basic, repeat questions since some newcomers go there before posting to the list. Occasional messages like the following suggest that individuals do avoid posting messages to the list because of the wiki, although most of the time they do not mention it on the list:

You can ignore my previous message (and this one, for that matter)... I found the wonderful Wiki at [FrontPage wiki URL] and have answered my own question already.

Thanks for such a great resource, Bill

Interviews with core members indicated that they believed the quality of questions improved because of the wiki. For example, one interviewee said that the wiki 
was useful in "encouraging long-term members like me to stick around, since we can move FAQs to the wiki and don't have to be bored or frustrated by those threads as often.” Although it is hard to definitively prove that fewer frequently asked questions arise on the list, in this case the members' perception is more important than the reality because it is their perception that influences them to stay or leave. Even when frequent questions do arise, the wiki helps to efficiently answer them (as described in the previous section).

In addition to dealing with FAQs, the wiki has also helped some active members who cannot spend the time reading all of the email messages to stay at least partially connected to the community. Two members mentioned in interviews that they continue to review and edit wiki pages even though they don't have time to subscribe to the email list any longer. By providing a new form of lower cost participation, the wiki has enabled experts to continue to share their knowledge through the wiki even if they don't through the list.

\section{Motivating \& Focusing Contributions}

Earlier we saw how the email list helped motivate contributions to the wiki. In this section we look at the opposite direction, how the wiki helps motivate individuals to contribute to the email list. This occurs when individuals explicitly mention that they will add contributions they receive in the email list to the wiki.

Below are some examples taken from different email list messages showing how members have used the wiki to help motivate higher quality email list contributions:

- Any extras or techniques beyond the simple things I've listed would be great. Any results I'll compile up and put on the css-d wiki.

- Can fellow listers help out here or point me to existing sources or examples? If we can assemble such a set of features I'll offer to put it up on the Wiki. 
- I'm sure this IE 6 bug has come up *many* times here before, but $i$ could not find anything about it in the wiki. I'll happily write something up and put it in if I can get some more info.

- (I had a quick look on the Wiki and couldn't see anything, so i'll add the solution there if it's possible to do this)

List members who read these messages are assured that their contributions will not only benefit the individual asking the question, they will also benefit all the people exposed to the wiki. One list member who does not himself contribute to the wiki mentioned that he had contributed through others by responding to these types of inquiries. This suggests that these promises have an effect.

As was previously described, this approach can be used to help avoid problematic discussion as well, when coupled with private messages. Thus, the wiki helps motivate members to contribute content (to individuals), while helping them keep the list policies (by posting only on-topic conversation to the list). The following example adds to the one provided in the prior section: “/Please/ don't answer this part of the thread. Any suggestions and critiques please send off list to me. I'll collect them and will bring it to the wiki if anyone thinks this could be of interest."

In addition to motivating contributions, the wiki has been useful in focusing attention on a particular topic and encouraging in-depth discussion of that topic on the email list. Several of the most frequently cited Article pages began as email list announcements intended to solicit ideas and see if the topic was of general interest. This helped motivate members to share their thoughts on the topic in the email list discussion. When pages were created and announced to the list, it prompted further in-depth discussion about the content. The following example shows how this cycle plays out:

Web designers want to make accessibility information heard but not seen. Reports about what this and that screenreader does are always helpful, but spotty. Which screenreaders pay attention to CSS, to linked CSS, to imported CSS, etc? Which is better display:none or visibility:hidden? 


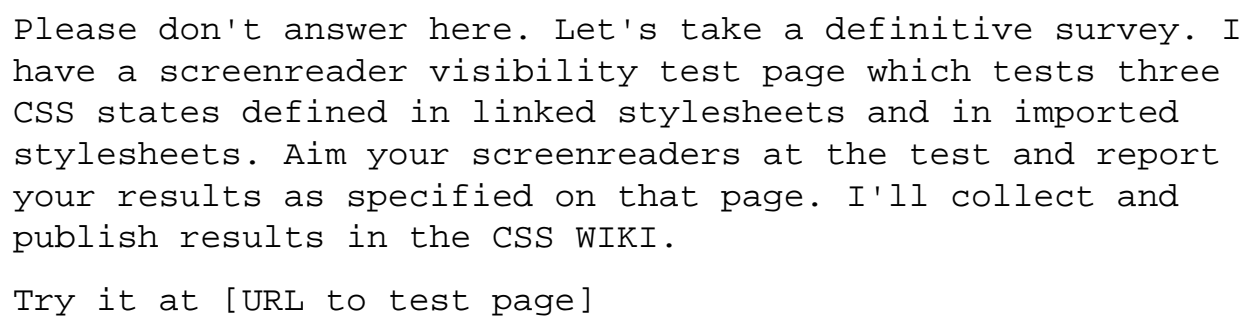

This message relates to the ability of CSS to work well with screenreaders used for visibly impaired individuals. After many people responded to this email the author created the ScreenreaderVisibility wiki page displaying the results in a table with some background and commentary. He announced the page to the list and had dozens of replies discussing the findings and proposing new solutions. This led to another round of testing (of a new technique proposed and refined by various list members) and further commentary. The editor continued to add content to the wiki based on the discussion, as did a few others. Several people indicated their gratitude for the collaborative work being done.

This example demonstrates how the wiki has been used to help motivate contributions, as well as focus attention on a particular topic. In this case, members were motivated to test pages and report the results so that a wiki page would be created summarizing them. Once it was created, it acted as a focal point for the conversation. Although people had discussed screen reader problems in the past (and how to get around them), they now had a working draft of a document explaining the key issues and proposed solutions. The work changed from a traditional Q\&A where people help a single individual, to a collaborative authoring challenge where the community helped to suggest to the world (via the wiki) the challenges and best practices in this area. This helped motivate and focus attention so that the email list discussion progressed past the typical question and answers. Other cases, such as the IE7 page, have helped focus the email list discussion on performing useful work of lasting value that otherwise might not have occurred in the list or by the community. 


\section{Conclusions}

In this chapter I have drawn attention to the synergies that exist between the email list and the wiki repository, as well as the social activities and roles that couple them together. Data show that the wiki is referenced in approximately 2 messages per day (4\% of all messages) and that it is referenced by members of all participation levels for a variety of purposes: to educate others (e.g., as when answering a question), ask a question, offload the discussion (e.g., avoid a holy war), discuss prior and future edits, give thanks, and discuss its proper use. The email list archive was referenced far less during the same time period (302 times versus 1,511 times) suggesting that the wiki is better suited for reuse. Over 112 of the 544 wiki pages were referenced in the email list conversation, with the top 13 pages accounting for half of the total wiki references.

The email list was found to influence the wiki in several ways. It helped provide a critical number of contributors, as well as a working governance structure that helped focus attention and establish norms around the wiki. It helped motivate contributions to the wiki, as when expert question answerers create pages on frequently discussed topics so they can later refer to them. It helped suggest topics of interest to the community, because of their frequent occurrence in the discussion. It provided content that could be copied and summarized in the wiki, as well as commentary on that content by other members who provided alternate ideas and peer review. And finally, it helped identify problems with wiki content (or lack of content), as when members came to the list after having tried to apply problematic CSS code from the wiki.

The wiki was found to positively influence the email list in several ways as well. It helped make question asking and answering easier because content could be reused (i.e., referenced) rather than recreated. It provided a place to safely offload problematic discussions (e.g., holy wars and off-topic conversation). It helped attract new members (since it showed up high on many search results) and helped them integrate into the 
community. It helped retain long-term members by lowering the number of repeat questions and reducing the cost of answering them. And finally, it helped motivate high quality email list responses, as when members promised they would summarize any responses they received into a wiki page.

As far as I know, this is the first empirical description of the work of coupling a wiki repository and email list together and the resulting benefits from such a relationship. It can be profitably used in a few different ways. First, it has helped describe some of the underlying processes, activities, and social roles that were key to the successful coupling of the repository and email list. These are characterized into suggested best practices in Chapter 7, and are validated in other communities in Chapter 9. Second, the analysis can be useful in determining the appropriateness of certain design choices. For example, a designer might suggest that it would be useful to automatically insert links to relevant wiki pages into email list messages. While this could indeed be the case, the current study points out that some pages are edited by experts because they must manually visit the page in order to copy the URL link. Recognizing this possibility, a designer may consider other methods that encourage experts to view and edit wiki pages when answering questions, rather than simply inserting relevant links. 


\section{CHAPTER 7}

\section{CSS-D PRACTICAL LESSONS LEARNED}

The past three chapters have provided an in-depth look at the email list and wiki and how they are coupled together. I have also argued that much of their success is derived from their close coupling, as well as other best practices that have been implemented by the community. This chapter is a summary of the practical lessons learned from the css-d study. They can be thought of as design suggestions in the broad sense of social and technical design. These lessons are prescriptive in nature and are intended to inform other online support communities interested in combining an email list and wiki repository. In fact, Chapter 9 will discuss how they were used to help develop the training materials (see Appendix 3) used to help several online medical support communities adopt a wiki repository. Each of the following sections discusses a suggested best practice.

\section{Seed the Wiki with Content}

The first recommended best practice is to provide some high quality initial content that will be immediately useful to community members before rolling it out to the community. In other words, seed the wiki with high quality content. As is discussed in Chapter 5, early members of css-d believed that seeding the wiki with high quality content helped encourage equally high quality contributions in the future. It also helped members get an idea of what the wiki repository could be used for (e.g., brief descriptions of frequently discussed topics and summaries of holy wars).

The css-d wiki took about a month to seed with content and included contributions from several members including the list administrators. Contributors had a 
range of CSS expertise (although they were likely more expert than the average list member) and a couple of them had experience contributing to other wikis. This combination seemed to have worked well, although no data on contributions from that period exists, making it impossible to see how this played out.

Finally, some individuals who seeded the wiki mentioned using the email list archives to help gather content for the wiki pages. As we saw more generally in Chapter 6 , the provision of topics and content from the discussion can help create wiki pages relevant to many people and can lower the cost of contribution. Thus, communities interested in adding a wiki to an existing email list conversation may consider drawing upon the email list archives when seeding the wiki.

\section{Establish Which Activities Should Occur in Each Resource}

The second suggestion is to establish which activities should occur on the email list and which should occur on the wiki. As I argue in Chapters 4 and 5, css-d has chosen to use each technology in a way that leverages its particular technical features. The email list, with its push technology, is used for time sensitive discussion. The wiki, with its pull technology, is used primarily as a resource upon which to draw and not as a place of interaction. The email list's emphasis on who authored messages and its chronological, threaded organization facilitate ongoing discussion. Activities such as asking and answering highly contextualized questions, announcing new browser releases, and getting feedback on draft websites are ideal for this setting. Meanwhile, the wiki's support for structured content, long pages, hyperlinks, and formatting facilitate document creation. Activities like aggregating and comparing information, summarizing (and decontextualizing) arguments and techniques, drafting comprehensive write-ups on subtopics, and refining existing pages are facilitated by the wiki technology. This natural combination of the two has worked well, so that the two resources work as complements 
rather than substitutes. In cases where different combinations were tried (e.g., the Site Check wiki pages), they were not successful.

An early recognition of the activities that should occur in each place by members seeding the wiki was important to its successful use. The content on the ToWikiOrNotToWiki page (see Chapter 5) suggests that early members at css-d understood the basics of which activities should occur where, although the failed attempt at the Site Check wiki system indicates that they did not have a perfect understanding. Their view of the wiki as an FAQ-like document helped them create pages that would be useful in the ongoing discussion (e.g., when answering questions). Their insightful use of the wiki to create Debate pages on contentious topics is one that may be overlooked by other communities, but has proven useful at css-d. Although not initially noticed as a useful thing, the inclusion of off-topic, but peripherally related content on the css-d wiki has been enormously useful. It has allowed members to divert some off-topic discussion on the list while still meeting members' needs. In addition, it has led to the creation of wiki pages that are among the most frequently referred to by email list participants (e.g., OffTopic and CssEditors) (see Table 6.2), as well as search engines (e.g., CssEditors) (see Table 5.2). All of these examples suggest that members should develop wiki content that serves the needs of the discussion, rather than try to create a comprehensive encyclopedia on the topic.

\section{Promote the Wiki Often Through Its Ongoing Use}

The third suggestion is to promote the wiki through its ongoing use and give it a prominent place in the community. Chapter 6 emphasized several ways that members coupled the two resources, many of which helped to reinforce the wiki's important role in the community and encourage others to participate in it. Below is a discussion of a few of the ways that css-d has successfully promoted the list to its members and the outside world. 
Official list policies suggested that members should search for answers to their questions on the wiki (and email archives) before posting to the email list. When administrators enforce these policies they also often reference specific wiki pages (e.g., they refer to the FontSize page when closing down Holy Wars on the topic).

The wiki URL was linked to from the main css-d website ("Css-discuss.org," 2002), as well as added to the footer of each list message with the following description: “List/FAQ - [URL]”. Later, when Internet Explorer 7 came out (a significant event in the website design community), a link to the IE7 wiki page was also added to the footer. Likewise, many community members link to the css-d wiki from their personal webpages. This is important because it helps people find it (indeed approximately $20 \%$ of visits to the wiki are from other websites not including search engines). It is also important because it increases the likelihood that it will show up highly in Google search results because of the Page Rank algorithm (Brin \& Page, 1998).

Members regularly link to specific wiki pages for a variety of reasons (see Figure 6.1 and Table 6.1). They link to it to ask and answer questions, perform administrative functions, announce new wiki pages, discuss problems with its content and prior edits, suggest future edits, and discuss its usefulness and place within the community. The correlation between the number of times a page has been referenced and the number of edits it has received is suggestive that this referencing is important in encouraging wiki contributions. The frequent links to the wiki also reinforce its existence to the many people listening in on the conversation. Having both novices and experts contribute to the wiki is important because each of them can identify different problems with the content. The fact that discussion of the wiki has never been considered off-topic is also telling (especially given that the discussion of most email list policies such as the reply-tosender default setting are considered off-topic). This suggests that the wiki is truly a community project where members can have significant input. All of this underscores the 
need to establish a strong norm of linking to specific wiki pages (rather than simply sending people to the wiki) and allowing people to openly discuss the wiki on the list.

Finally, Chapter 6 discussed the importance of the wiki as a resource for helping people find the community and learn about it initially. Server logs suggest that most people come to the wiki from search engines, some of which join the community. This suggests that the wiki repository should be open to the public at large if it is to help attract members.

\section{Establish Wiki Related Community Roles}

The fourth suggestion is to establish community roles related to the wiki. The experience of css-d suggests what a few of these roles might be, although they have not officially recognized these roles (see Chapters 5 and 6). Bridge Builders were identified as core email list community members who helped transfer knowledge between the two resources. They must be active participants in both communities to fulfill this role. They help identify topics that would be good ones for the wiki, contribute content, frequently reference the wiki, and encourage others to add content to the wiki. Two types of Bridge Builders are discussed in Chapter 6: Wiki Advocates and Wiki Page Editors. Wiki Advocates keep up-to-date on their knowledge of wiki pages and frequently reference them, as well as encourage others to reference them when appropriate. They also recognize new opportunities for using the wiki by identifying frequently discussed topics or debates and encouraging others to create pages related to them. More generally, they encourage people to read the wiki often. Page Advocates act as editors of specific pages. They solicit input from the community on the topic, create a page (typically an Article wiki page), refer to the page in the email list, and then update the page based on the discussion and new insights they gain from other sources.

Another group of contributors, Wiki Experts, was identified as experts related to the use of the wiki, although not necessarily CSS. They help organize content, structure 
documents, and generally “clean up” the wiki. Finally, a few individuals (Spam Hunters) help remove spam from the wiki, a task that requires no CSS knowledge and may be performed by novice lurkers on the email list as a way of giving back to the community.

The most common complaints from interviewees about the css-d wiki content were that some of it is outdated, disorganized, or not as "polished" as it should be. This is likely because many of the most active contributors are Bridge Builders rather than Wiki Experts. For example, only a few members mentioned using the RecentChanges page regularly at css-d, whereas members in other wiki communities such as Wikipedia rely heavily upon the RecentChanges feature. The establishment of a new Clean Up Crew role may be desirable, and was recommended by two interviewees. Individuals who are in the Clean Up Crew would help delete outdated content (which rarely happens at css-d), identify pages that need updating, help organize content, and improve consistency and readability throughout the wiki. The official recognition of such a position may make this work more apparent to the other community members who may not be aware that it is occurring. This may in turn act as a motivator for members to perform these forms of work. Tools that aided them in these efforts could also be useful. For example, highlighting old text, unused pages, or even sending out automated reminders about updates could benefit members.

\section{Consider \& Support Wiki Genres}

The fifth suggestion is to actively consider which wiki genres would be most appropriate for certain situations and support their use. Chapter 5 outlined several wiki genres that have been particularly useful to the css-d community, such as the Debate, Annotated Links, Article, and 'How To' genres. Some of these pages were created from the start, while others came about later. There is evidence that the existence of a certain genre helped inspire others to create new pages of a similar type. For example, the ThreeColumnLayouts page was the first Annotated Links page dealing with layouts. 
After it became well known, several other similar pages (e.g., TwoColumnLayouts page) were created. It is likely that seeing one page of a particular genre may help members to see other possibilities of topics that would fit well with the same genre. In this way, members of other communities may benefit from being able to see a palette of wiki genres that have been useful in online support communities.

\section{Be Patient}

The sixth and final recommendation is to be patient when introducing a wiki repository. The experience of css-d suggests that members starting a wiki repository should expect it to take some time. Statistics presented in Chapter 5 suggest that the wiki has grown at a relatively slow, but steady pace of only a few sentences a week. Although there are likely hundreds and possibly thousands of people who have edited the wiki, initially it was primarily the work of only a handful (i.e., those who seeded it). In fact, one member sent an email to the list expressing his concern that the wiki pages he had seen were often the work of just 2 or 3 people. And this was after the wiki had been around for several months. This suggests that communities implementing a wiki repository should not give up too early if numerous individuals do not contribute. Instead, they can continue to actively promote the wiki (as described above) and celebrate small but significant improvements.

\section{Summary}

Table 7.1 summarizes these suggestions.

Table 7.1: Suggestions for Implementing a Wiki Repository

\begin{tabular}{|l|l|}
\hline Suggestion & Details \\
\hline Seed the wiki with content & $\begin{array}{l}\text { Make sure initial content is high quality } \\
\text { - }\end{array}$ \\
& $\begin{array}{l}\text { Cone active list participants and individuals with wikitially experience } \\
\text { Use the email archives to suggest what topics should be addressed } \\
\text { and to gather content }\end{array}$ \\
\hline
\end{tabular}




\begin{tabular}{|c|c|}
\hline $\begin{array}{l}\text { Extablish which activities } \\
\text { should occur in each } \\
\text { resource }\end{array}$ & $\begin{array}{l}\text { The threaded discussion is better for addressing immediate needs, } \\
\text { things of little lasting value, social activities, announcements, and } \\
\text { is a place of last resort. } \\
\text { The wiki repository is better for capturing content of lasting value; } \\
\text { summarizing, decontextualizing, and aggregating information; } \\
\text { outlining debates; and including off-topic, but related content. }\end{array}$ \\
\hline $\begin{array}{l}\text { Promote the wiki often } \\
\text { through its ongoing use }\end{array}$ & $\begin{array}{l}\text { - Link to it in the email list footer and other websites } \\
\text { Encourage its use in the list policies and link to wiki pages when } \\
\text { enforcing policies (e.g., when closing down a Holy War) } \\
\text { Establish a norm of linking to specific wiki pages when asking and } \\
\text { answering questions } \\
\text { Encourage discussion of wiki content on the email list (e.g., } \\
\text { announcements of new pages, suggestions for what should be } \\
\text { added, potential problems with a page) } \\
\text { Make the wiki repository open to the public, not just the email list }\end{array}$ \\
\hline $\begin{array}{l}\text { Establish wiki related } \\
\text { community roles }\end{array}$ & $\begin{array}{l}\text { Wiki Advocates who are active list members and ideally content } \\
\text { experts so they can recognize what needs to be added to the wiki } \\
\text { and have the credibility to suggest that others add it. They should } \\
\text { also be proficient at using the wiki so they can make their own } \\
\text { additions. } \\
\text { Wiki Page Editors who are active list participants and content } \\
\text { experts on a sub-topic of the community topic. They help create } \\
\text { and maintain Article pages by soliciting input from the } \\
\text { community, contributing their page(s), referencing their page(s) in } \\
\text { the email list, and updating it. } \\
\text { Clean Up Crew members who are wiki experts. They can help } \\
\text { orgainize the content, format it consistently, and delete (or at least } \\
\text { identify) outdated content and spam. }\end{array}$ \\
\hline $\begin{array}{l}\text { Consider and support wiki } \\
\text { genres }\end{array}$ & 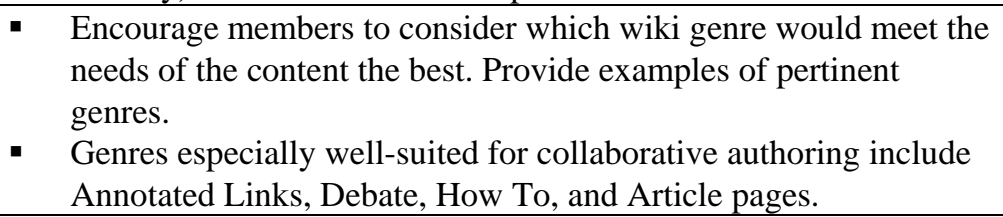 \\
\hline Be patient & $\begin{array}{l}\text { - } \quad \text { Establish realistic expectations for growth and community activity } \\
\text { - Celebrate minor milestones }\end{array}$ \\
\hline
\end{tabular}




\section{CHAPTER 8}

\section{ONLINE MEDICAL SUPPORT COMMUNITIES}

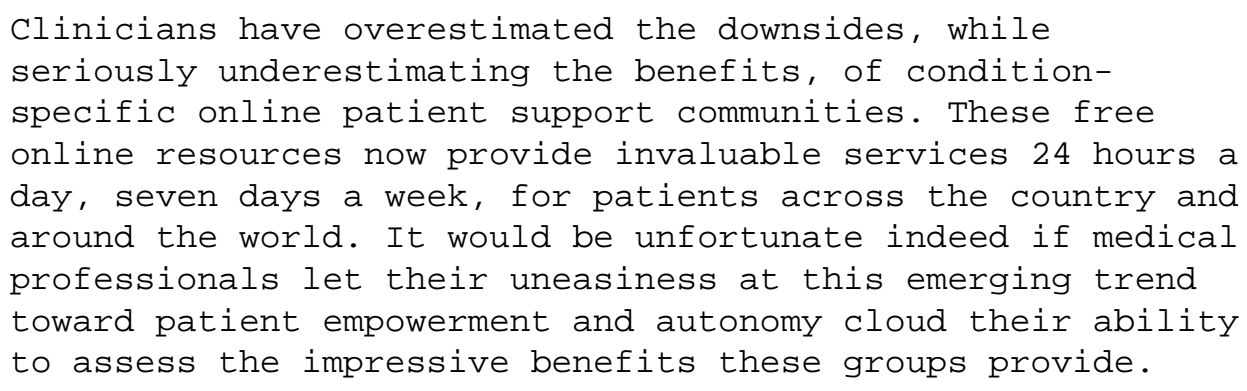

(Hoch \& Ferguson, 2005)

The purpose of this chapter is to introduce a sub-type of online support

communities, namely online medical support communities (OMSCs) and to contrast them with the technical support community (css-d) discussed in Chapters 3-7. This comparison is important in translating the proposed best practices from the css- $d$ study (see Chapter 7) into the medical support community context. I also use this chapter to identify the unique challenges and consideration of OMSCs when introducing a wiki repository, as well as discuss important design considerations when working with OMSCs. The following sections are included in this chapter:

- OMSC Overview

- Advantages \& Disadvantages of OMSCs

- Differentiating Factors of OMSCs

- Activities

- Comparison of OMSCs and CSS-D

- Design Oriented Studies

- Discussion 


\section{OMSC Overview}

Since the early days of the Internet, individuals have participated in OMSCs (Ferguson, 1996). Like all online support communities, OMSCs are “distributed, computer-mediated communities of practice focused on supporting the needs of individuals who are trying to make sense of, and deal with, a particular situation or knowledge domain” (Chapter 2). They are differentiated from other online support communities in that their support topic relates to a medical condition (e.g., diabetes, depression), health-related behavior (e.g., weight loss, smoking cessation), or other health-related issue (e.g., the use of a medical device such as a g-tube) from the perspective of a patient (see Chapter 2 for a discussion of support topics). This patientcentric, medical focus often leads to similar activities (e.g., sharing social support), communication styles (e.g., empathetic), and concerns (e.g., dealing with complex medical terminology). Thus, they are an important subset of online support communities that share important characteristics with one another.

It is hard to estimate the total number of OMSCs, but they are clearly numerous. For example, over 38,000 Yahoo Groups were listed in the "Health \& Wellness > Support” category in June 2007. Another popular collection of online support communities, ACOR.org sends out more than 1.5 million emails a week (Ferguson, 2007). A recent Pew Internet and American Life survey estimates that 79 million Americans have participated in an online support group (Rainie, 2005). Many of them

reach even more individuals through content such as email list archives that are indexed by search engines.

The popularity of OMSCs has attracted significant attention from patient advocates, and medical professionals, policy makers, and the media. Advocates suggest that they are an important new way of empowering patients and countering the strong power structure of existing medical practice (e.g., Ferguson, 2007; Hoch \& Ferguson, 
2005). Meanwhile, medical practitioners are concerned about the quality of information shared within these communities (e.g., see Culver, Gerr, \& Frumk, 1997; Esquivel, Meric-Bernstam, \& Bernstam, 2006), as well as their potential at improving health (Eysenbach, Powell, Englesakis, Rizo, \& Stern, 2004). Policy makers have examined the implications of the rise of OMSCs on professional-client relationships and broader social policy issues such as social exclusion (Burrows, Nettleton, Pleace, Loader, \& Muncer, 2000). All of this attention suggests that OMSCs are already an important information resource to many, despite their relatively recent appearance on the scene.

\section{Advantages \& Disadvantages of OMSCs}

A great deal of literature has discussed the benefits and disadvantages of OMSCs (e.g., Ferguson, 2007; Rimer et al., 2005; Shaw, McTavish, Hawkins, Gustafson, \& Pingree, 2000; White \& Dorman, 2001). These are summarized below:

\section{Advantages}

- Accessibility. They are available 24-7, even from many remote areas where traditional care is limited. Likewise, they are conveniently available from home for many individuals. This is especially important for those who have diseases that impede travel. Their free availability (or in some cases low cost) also makes them more accessible.

- Unique Source of Information. They make finding a critical mass of participants for rare diseases possible, providing individuals with access to peer-provided information that would otherwise be unavailable. They also encourage a holistic approach to care, where not only the immediate medical concern is discussed, but also its implications on all aspects of life and day-to-day management issues (e.g., what to say to the school nurse about your child's medical problem; where to buy cheap medical supplies). This type of information is often lacking from medical professionals and official information resources. They can also be helpful in keeping up on the state-of-the-art treatments and research on one's condition. Members often share recent research articles and talk about where they received new treatments and how effective they were. Finally, because of the ability to post anonymously, members can freely discuss some topics (e.g., sexual dysfunction) that can be embarrassing to talk about in person. 
- Social Support. Because members find others with common experiences, they are able to empathize and provide social support in ways that members' immediate social networks often cannot. They play an especially important role in the lives of individuals who lack strong social networks in the real world. Members also continue to provide support for the incurable, even after other organizations or social networks cannot.

- Interactive and Personalized. Their interactive nature encourages people to become more involved with their care and follow through with it (Neuhauser \& Kreps, 2003). This interactivity also allows individuals to receive customized and contextualized answers to their questions, unlike static information resources.

\section{Disadvantages}

- Deviant Behavior. The potential for anonymous posting allows for deception and flaming.

- Privacy Concerns. When members share personal information it is often made widely accessible (and searchable) via the Internet, which can cause privacy concerns.

- Limited Medium. The text-only medium can make certain types of social support (e.g., hugs) and information sharing (e.g., showing how to clean a medical device) difficult or even impossible. It can also increase misinterpretations of statements, causing discomfort and anxiety, since many non-verbal cues are missing.

- No Quality Assurance. Information is not typically reviewed by medical experts and therefore, may contain errors. However, in some communities these are caught by other members and it is not clear whether or not members act upon misinformation (Esquivel et al., 2006).

- Accessibility. They are not available to some populations including those without access to a computer (much of the world's population) and knowledge of how to use it (e.g., ability to read and write, use a keyboard, and email).

Although members have consistently self-reported positive experiences from

OMSCs (e.g., Ferguson, 2007; Shaw et al., 2000), not enough randomized control trials have been conducted to demonstrate their effectiveness at improving health and psychological well-being (Eysenbach et al., 2004). Despite this lack of evidence, their widespread use and the long-term participation of many members suggests that at least some individuals benefit from their participation in them. 


\section{Differentiating Factors of OMSCs}

Although OMSCs share many similar characteristics, they also differ across several dimensions. It is important to recognize these differences because they can affect the community activities that occur, the community focus, the membership, and the technical solutions that are helpful. As with all online support communities, OMSCs differ in their support topic, community size, membership duration, member diversity, and their approach to social maintenance (see Chapter 2 for a discussion of these factors). Here we emphasize two particularly important factors specific to the medical realm that differentiate OMSCs from one another: sponsorship and the nature of the medical condition.

\section{Sponsors}

Who sponsors a particular OMSC can have a significant effect on its membership, focus, technological infrastructure, and patterns of communication. Many OMSCs are run by patients using free groupware such as Yahoo! Groups and Usenet, sites set up and paid for by volunteer members, or sites that fund themselves through advertisements (e.g., Mediboards). Other groups are associated with a patient advocacy group (e.g., The Endometriosis Association; GIST Support International) that raise awareness of a particular disease, educate patients, and raise money for research and community activities. These groups often have websites with expert-provided content and connections to medical researchers (e.g., www.gistsupport.org), although their OMSCs are often run by patient volunteers. Both of these types of groups are very patient-centric.

Other sponsors include hospitals (e.g., Hartford Hospital who offers CHESS) ("CHESS - Comprehensive Health Enhancement Support System," n.d.), pharmaceutical companies (n.a., 2000), researchers, and private firms (e.g., RevolutionHealth, WebMD). These communities are more likely to have experts that monitor the conversations. They 
also are more likely to have discussions where patients are encouraged to "ask an expert." Some researchers have encouraged the medical system to embrace OMSCs (Johnson \& Ambrose, 2006) in order to help facilitate the downstream health and cost benefits. However, those with extensive experience working with patient-driven communities warn that professionally-run groups are very different from patient-initiated groups and may discourage patient initiative, assertiveness, and empowerment (Ferguson, 2007). For example, one of the communities I have worked with split off from another community because it felt that the other community was too closely associated with the pharmaceutical industry. They were worried this connection would inhibit members from an open dialogue. More generally, the sponsor influences which communities members choose and their actions within those communities.

\section{Nature of Medical Condition}

Chapter 2 already discussed how communities differ based upon the support topic. Here I re-emphasize this point by emphasizing that different medical conditions can influence many factors of the OMSCs. For example, researchers have found that the demographics of the members and the prevalence of certain activities (e.g., seeking information; social support) can differ based on illness (Davison \& Pennebaker, 1997). For example, prostate cancer discussion forums will naturally include more men than women, while breast cancer forums will include more women. Other diseases affect individuals within certain age ranges. These demographics may alter the activities, as women are more likely to share social support and men are more likely to share information (Klemm, Hurst, Dearholt, \& Trone, 1999). The duration of membership may also be influenced by diseases, with chronic conditions encouraging long-term membership more than acute conditions. The rarity of a condition also has implications on the size of a community and the amount of outside resources available to group members. 


\section{Activities}

The majority of research on OMSCs has been descriptive in nature. Many studies have examined the primary activities that occur in OMSCs (see White \& Dorman, 2001 for a literature review of early studies). These studies were based on content analysis of messages from OMSCs. Because different coding schemes were used in different studies, it is difficult to directly compare the prevalence of various activities. However, despite the differences in the specific categories, several common activities were found. Although individual communities differ in their proportion of these activities, they were found in nearly all OMSCs studied. The major activities include sharing of social support (e.g., emotional support, instrumental support), sharing information, and maintaining social order (e.g., keeping the discussion on topic and avoiding contention).

\section{Social Support}

Several studies have emphasized the importance of the provision of social support within OMSCs (e.g., Meier et al., 2007; Shaw et al., 2000; White \& Dorman, 2000; Wright, 2002). The provision of social support in offline settings has been shown to improve patients' quality of life and buffer the impact of stressful life experiences (Cohen \& Wills, 1985; Cutrona \& Russell, 1990), increase sense of self-esteem (Metts \& Manns, 1996), and help patients effectively cope with problems (Kohn, 1996). Epidemiological studies have also shown that social support is associated with positive health outcomes and the lack of it (i.e., social isolation) is associated with negative health outcomes (e.g., Blazer, 1982; House, Landis, \& Umberson, 1988).

Several types of social support have been characterized in the literature, such as emotional support, informational support, and instrumental support (Helgeson \& Cohen, 1996; Meier et al., 2007; Shaw et al., 2000). Informational support involves the “provision of information used to guide or advise” (Helgeson \& Cohen, 1996, p. 135) and 
is discussed below under the Information Sharing section. Emotional support is the demonstration of caring and concern for others through acts such as "listening, 'being there,' empathizing, reassuring, and comforting” (Helgeson \& Cohen, 1996, p. 135). It can help improve restore self-esteem and help individuals express feelings that may reduce stress (Helgeson \& Cohen, 1996; Meier et al., 2007). It may also help individuals recognize purpose and meaning in the disease experience (Helgeson \& Cohen, 1996). Finally, instrumental support involves "the provision of material goods, for example, transportation, money, or assistance with household chores” (Helgeson \& Cohen, 1996, p. 136).

Empirical studies have found that all of these forms of support occur in OMSCs, although in differing levels. We discuss informational support in the following section on Information Sharing. Here we discuss emotional support and instrumental support.

\section{Emotional Support}

Empirical studies have consistently found that many messages provide emotional support, either as the sole purpose of the message, or as an aside to other activities occurring in the message (i.e., such as answering a question). It is hard to compare numbers across studies since different definitions were used. Meier et al. found that emotional support messages ranged between $10 \%$ and $30 \%$ of all messages in 10 different ACOR groups (2007). This is consistent with other studies in that range (e.g., Klemm, Reppert, \& Visich, 1999b; Miller \& Gergen, 1998; White \& Dorman, 2000; Winzelberg, 1997). Other researchers have found higher numbers. For example, Braithwaite et al. found 40\% emotional support messages and another 19\% esteem support messages and Preece categorized 45\% as empathic (1999b). Other studies that use inductive coding methodologies also found social support to be a major theme (e.g., Dickerson, Flaig, \& Kennedy, 2000; Sharf, 1997; Shaw et al., 2000). 
There are different forms of emotional support that occur online. Meier et al. found that expressions of encouragement and empathy were most common, followed by sharing of emotional coping strategies, prayers, esteem support, and solidarity (2007). Preece found no evidence of non-empathic communication (1999b). Pfeil and Zaphiris describe several types of "light support" including encouragement, humor, showing interest, giving best wishes, as well as types of "deep support” such as providing reassurance, giving help, and providing deep emotional support (2007). Fernsler \& Manchester emphasize the importance and prevalence of expressing hope and optimism as a form of emotional support (1997).

Both Meier et al. (2007) and Pfeil and Zaphiris (2007) found that explicit requests for emotional support are rare in OMSCs, despite the fact that offers of support are frequent. Instead, members self-disclose information about themselves by sharing their general feelings, stories, and details about their medical condition, which then leads to expressions of emotional support by others. Self-disclosure is a key activity in many OMSCs (e.g., Dickerson et al., 2000; Klemm et al., 1999b; Miller \& Gergen, 1998; Pfeil \& Zaphiris, 2007; Salem, Bogat, \& Reid, 1997; White \& Dorman, 2000; Winzelberg, 1997) . It is integrated into other community activities such as information sharing, but is especially important at initiating the provision of social support. For example, Pfeil and Zaphiris point out that self-disclosing general feelings and medical situations often trigger empathic responses (2007). In fact, they found that over $70 \%$ of all messages included self-disclosure (Pfeil \& Zaphiris, 2007). Thus, emotional support is sought after through telling stories and describing feelings rather than asking questions or asking for help explicitly. These narratives and explicit descriptions of feeling are necessary in an online context because the other members are not able to deduce them from direct observation (Pfeil \& Zaphiris, 2007).

\section{Instrumental Support}


Instrumental support (i.e., the provision of physical or monetary help) is the least common form of support offered online. While giving rides to the doctor or babysitting for other members may not be possible in distributed settings, many forms of instrumental support such as providing medical supplies, introducing a member to a specialist, and sending money are all possible from afar. Braithwaite et al. (1999) and Salem et al. (1997) found that the provision of tangible assistance was rare but regularly occurred. Perhaps because of the nature of the community, Hansen observed the relatively frequent sharing of medical supplies among members of a community of g-tube users (2003). Members also send monetary support to family members of long-time participants who have passed away.

\section{Information Sharing}

Studies have found that information sharing (i.e., informational support) is the primary activity occurring within many OMSCs. For example, the Health eCommunities Project found that the main reason members join ACOR groups is to gain information (e.g., 63\% strongly agreed that one of the reasons they joined the mailing list was "to find out about the latest treatments for cancer”). Emotional support was another reason, but to a lesser degree (42\% strongly agreed that they joined the mailing list to "get support from other people with my cancer”) (Rimer et al., 2005). Later content analysis of messages from 10 ACOR groups suggested that information sharing was the most common activity (between $70 \%$ and $90 \%$ of all messages), with emotional support prevalent but less common (Meier et al., 2007). Earlier studies found information sharing to be important, but not as prevalent ranging from $17 \%$ to $32 \%$ of the messages (Braithwaite et al., 1999; Klemm et al., 1999b; Preece, 1999b; White \& Dorman, 2000; Winzelberg, 1997). This suggests that the balance of information sharing versus emotional support varies considerably, but both are key activities of OMSCs. Other studies that used a more 
inductive coding method also found information sharing as one of the primary activities (Dickerson et al., 2000; Sharf, 1997; Shaw et al., 2000).

Like in other online support communities, information sharing occurs primarily through questions and answers. These are highly contextualized and often based on the immediate needs of the question asker (White \& Dorman, 2001). For example, members ask about treatment options they need to decide between, how to prepare for a surgery, what side effects they should expect to have from a procedure or medication (e.g., chemotherapy), what to say to their doctor, and a host of other practical issues related to coping with a medical issue (e.g., how to prepare blended food when traveling without a blender). Answerers often refer to personal experience as a way of lending authority to their suggestions (e.g., Galegher et al., 1998). When sharing personal experiences, members describe their actions, explain why they made (or didn’t make) certain choices, and evaluate their choices (e.g., "I’m really glad I chose to...because...”). Answerers also frequently recognize that their own experiences may not perfectly apply because of differences in personal characteristics and circumstances (Galegher et al., 1998). In addition, references to other online sources, many of which are from medical experts, are common in some OMSCs (Wikgren, 2003). These are referred to in order to educate, as well as to support an individual's position in a debate (Wikgren, 2001).

In addition to the question and answer discussions, information is announced and discussed by the group (Burnett, 2000; Wikgren, 2003). Announcements may introduce a relevant new medical journal or news article or a new personal discovery (e.g., "I finally figured out a way to keep my son from being constipated”). Burnett has described how announcements and general discussions about topics in online communities help support the use of the community as an "information neighborhood" (Marchionini, 1995) where “orienting information seeking” (R. Savolainen, 1995) can occur. In other words, people keep connected to the most recent developments in their area through announcements and overhearing others’ conversations. 


\section{Social Maintenance}

Like other online support communities, OMSCs must deal with various social maintenance issues. Most OMSCs have list owners/administrators who help maintain social order, but as in many online communities much of the social maintenance is performed through group enforcement. Burnett \& Burkle compared two medical newsgroups and coded for several "hostile interactive behaviors," as well as collaborative behaviors (2004). They found instances of flaming, trolling, and spamming in both communities, although their prevalence was ranged from less than $1 \%$ of messages in one group to $30 \%$ of messages in another group (counting responses to flaming). Another study of 10 ACOR cancer email list groups discussed the important role of list administraters (Meier et al., 2007). The authors describe how administrators model and encourage appropriate behavior (e.g., follow policies in their own posts; offer praise offline), keep the discussion on-topic (e.g., limiting “cute stories” not related to the topic), and enforce group norms (e.g., shut down inflammatory comments and commercial content; encourage trimming of posts). Maloney-Krichmar and Preece found that strong group norms were sufficient to deter most social maintenance problems without regular administrator attention in one OMSC (2005). Wright found that "off-topic/hostile remarks" was the second most frustrating disadvantage of using an OMSC, with the first being “delayed feedback” (2002).

\section{Other Activities}

A few other activities occur regularly, but less frequently in many OMSCs. Many researchers describe humorous messages (Braithwaite et al., 1999; Klemm et al., 1999b; Sharf, 1997; Shaw et al., 2000), although not all communities encourage them (e.g., see Meier et al., 2007). These messages are used for various purposes, one of which is provision of social support (Shaw et al., 2000). Community and relationship building 
activities such as networking have been found (Braithwaite et al., 1999). Finally, some OMSCs include artistic expressions such as poetry, artwork, and journal entries (Braithwaite et al., 1999).

\section{Comparison of OMSCs and CSS-D}

In this chapter I have introduced the goals and activities of OMSCs, which I consider to be a subset of online support groups. As discussed in the Introduction, I am ultimately interested in translating the findings from the css-d study into the medical support context. To do this, it is necessary to compare the css-d community with OMSCs. This helps provide a better understanding of which design suggestions gleaned from css-d will be most applicable, as well as other issues that should be considered when introducing a community repository to OMSCs.

\section{Similarities}

In Chapter 2, I suggested that all online support communities (including css-d and OMSCs) are distributed, computer-mediated communities of practice focused on supporting the needs of individuals who are trying to make sense of, and deal with, a particular situation or knowledge domain. I also described several shared characteristics of online support communities. In combination, these suggest that css-d is similar to OMSCs because they are all:

- distributed and computer-mediated,

- communities of practice (i.e., must include a joint enterprise, mutual engagement, and a shared repertoire),

- focused on supporting the needs of a group of individuals as they relate to a specific knowledge domain,

- use a threaded conversation design, 
- rely primarily on voluntary participation (including a power-law distribution of participation and many lurkers),

- and are public or semi-public.

In addition, css-d shares several other characteristics with OMSCs as described below:

- Highly contextual and personalized conversation. Both types of communities include a significant amount of question and answer activity. Questions are often asked in highly contextualized ways based on immediate information needs. They are also answered in very personalized messages that often take into account the specific circumstances of the individual in question (e.g., his content expertise, his emotional state). This is largely due to the complexity of the respective domains.

- High variability in expertise among members. Since both technical and medical domains are complex, individuals have a high range of expertise levels. Most OMSCs and css-d attract individuals all across this expertise spectrum.

- Reuse of prior conversation. Although few studies have directly looked at the issues of reuse of conversation, many studies of OMSCs mention as an aside that members are encouraged to read email archives or prior posts.

- Many similar activities. Many similar activities occur including asking and answering questions, announcing new developments, maintaining social order, getting second opinions, debating the merits of a particular action, and developing friendships.

- Welcoming of newcomers. Most OMSCs are very empathetic in their conversations and welcoming of newcomers, who are often newly diagnosed individuals who they see as particularly vulnerable. Although there is less emotion support at css-d (see next section), the emphasis on welcoming newcomers and showing them respect is similar.

\section{Differences}

Despite the many similarities, some significant differences exist between css-d and OMSCs. These are primarily driven by differences in the domains of interest. Website design is visual in nature and is based on code, unlike OMSCs which relate to individuals' health and personal well-being. This leads to several important differences as outlined below: 
- Consequence of errors. The potential benefits and harm caused by medical information is often greater than that of website design. Following bad advice in website design may temporarily damage a website, while following bad advice in some medical situations may cause irreversible damage to one's body - a problem that most people are far more concerned about. Galegher et al. believe this is the main reason why Usenet health support groups spent so much more effort establishing authority than other hobby-related Usenet groups (1998).

- Validating information. The quality of information is often harder to validate in the medical domain. For example, a piece of CSS code can be immediately tested with little to no risk to the designer. In contrast, some medical suggestions (e.g., change of diet) take a long time to go into effect and are difficult to relate directly to outcomes. This can contribute to the difficulty of individual decision-making and the need to rely on outside expertise (e.g., doctors, medical journals) in the medical realm (Galegher et al., 1998).

- Transferring information. The nature of the work also determines how difficult it is to transfer information from one person to another. At css-d, the topic of interest is an information product, CSS code, which can be shared directly with others. In medicine, the topic of interest is not an information product, making it difficult to transfer all that is necessary to accomplish certain goals. Medications and surgical procedures cannot be emailed around (like CSS code is), although information about them can be. This suggests that some activities that are possible at css-d are impractical in many medical contexts. While a member of css-d can directly review another member's website (as is done in Site Checks), a member of a cancer group cannot directly review another member's physical well-being without seeing her in person - although some measures of health (e.g., test results) can be shared.

- Personal stories and self-disclosure. Members of OMSCs often share personal stories and information about their medical condition. For example, members post regular updates on their health after doctor visits or vent about recent occurrences and their current emotional state. They also include signature lines stating their own diagnosis, common medications they are taking, and medical treatments they've received. Although people at css-d share stories of their battles getting clients to accept CSS designs and vent about browser problems, such occurances are relatively rare. The high frequency of personal stories and information that is shared in OMSCs can be attributed to two different things. First, personal accounts are such an integral part of giving and receiving emotional support. Second, the challenges of transferring information (see above) and concerns about giving someone false medical advice encourage members to share their own experiences even when answering questions (e.g., Galegher et al., 1998; Meier et al., 2007; Shaw et al., 2000). The personal information provides important contextual cues for the readers to determine if the author's experience is applicable to the reader's experience (Galegher et al., 1998). It also contributes to a sense of community (Galegher et al., 1998). 
- Privacy concerns. The risks associated with others knowing about your personal information are likely less at css-d than in many OMSCs. Although it is difficult to quantify this, the perception of privacy being an issue is certainly larger in many OMSCs.

- Amount of emotional support. As described above, sharing emotional support is a common activity in many OMSCs; far more common that at css-d. This is not to suggest that it was nonexistent at css-d, especially in the early years of the list due to the marginalized position of CSS in website design. Thus, expressions of encouragement and commonality (e.g., "I can't stand table layouts either) were fairly common, although not at the level of many OMSCs. And some types of support, such as mentioning praying on others behalf were nonexistent at css-d. One implication of this is that some OMSCs may be more willing to allow offtopic discussion of a personal nature in order to allow relationships to be strengthened, although certainly not all OMSCs do (Meier et al., 2007).

- Technical expertise of members. Because website design requires technical expertise, many members of css-d are easily capable of learning to use new technologies (e.g., a wiki). Although there may be a few technical experts in OMSCs there is not the same selection bias that encourages technical experts to participate, especially in groups that use basic software such as email.

\section{Design Oriented Studies}

Most empirical studies of OMSCs are published in medical, communications, and sociology journals and discuss implications of OMSCs on health education (e.g., White \& Dorman, 2001), health outcomes (Eysenbach et al., 2004), specific diseases (Lasker, Sogolow, \& Sharim, 2005), healthcare policy (Burrows et al., 2000), health communication (Wright, 2002), and patient involvement (Hoch \& Ferguson 2005). Fewer studies are published in design-oriented publications and examine social and technical design considerations of OMSCs (e.g., Leimeister \& Krcmar, 2005; Maloney-Krichmar \& Preece, 2005; Preece, 1998), although there is continued interest in improving the design of OMSCs (Neal et al., 2006; Neal et al., 2007). The design considerations raised in these articles are important to consider because they help identify issues unique to OMSCs.

\section{Facilitating Emotional Support and Information Sharing}


Several studies have emphasized the need to provide social and technical support of empathic communication and emotional support more generally (Pfeil \& Zaphiris, 2007; Preece, 1998). Suggestions include providing members with the ability to create personal profiles (Pfeil \& Zaphiris, 2007) that can be used to find similar people (Leimeister \& Krcmar, 2005; Preece, 1999), making sub-group creation possible (Maloney-Krichmar \& Preece, 2005), making private communication via email possible (Preece, 1999), providing synchronous communication opportunities such as scheduled chats (Wright, 2002), allowing members to represent their emotional state (e.g., through various smiley faces (Maloney-Krichmar \& Preece, 2005), and improving moderation techniques (Meier et al., 2007; Preece, 1998). Leimeister \& Krcmar offered personal profiles in an OMSC for cancer patients (2005). They found that core members used personal profiles regularly by posting messages on each other's "guest books." These messages often included social support comments. However, many members (43.1\%) did not allow other members to view their own personal profile. In general, members who revealed more information received more comments in their "guest books" suggesting that there may be a tradeoff between encouraging emotional support and privacy concerns. They also found that members rarely used the "contact search" feature to look for other members. Instead, they visited pages of people they already knew from the discussion, which included a relatively small number of people during the study.

Preece argues that there is a tradeoff between facilitating emotional support and information sharing and that designers must also be wary of disrupting one by facilitating the other (1999). For example, she warns that sending newcomers to an FAQ or inviting medical experts to participate may discourage the sharing of emotional support (Preece, 1999). My own experience with OMSCs suggests that some communities allow off-topic conversation when it is likely to strengthen relationships among group members, and thus the ability of members to empathize with one another effectively. However, this is often done at the expense of those primarily interested in the information sharing activities. 
Shaw et al. suggest that smaller communities are needed for emotional support to thrive (2000), but smaller communities have access to fewer information resources, making them less useful for information sharing. These tradeoffs suggest that there is a need to "examine design solutions to support empathic communication, so that it can co-exist well with factual communication exchanges” (Preece, 1999).

\section{Establishing Trust}

Establishing trust in the community is an especially important design consideration for OMSCs (Leimeister, Ebner, \& Krcmar, 2005; Maloney-Krichmar \& Preece, 2005). There is considerable research on establishing trust in corporate environments (e.g., Shneiderman, 2000), but less research that is specific to OMSCs. Leimeister and Krcmar (2005) suggest that two major factors in establishing trust are perceived competence and perceived goodwill (see also Abdul-Rahman \& Hailes, 2000; Ebner, Leimeister, \& Krcmar, 2004).

Perceived competence is especially important in OMSCs because of concerns about the quality of health information. Leimeister \& Krcmar recommend having trusted third parties provide certification that certain best practices are being followed (2005). For example, the Health On the Net Foundation provides an accreditation process for health websites, which must indicate the qualifications of authors. Other online communities rely on recommender systems where members rate the quality of messages (Resnick \& Varian, 1997), although some authors have suggested that such practices may disrupt the emotional support in OMSCs (Preece, 1999). Wright (2002) and MaloneyKrichmar and Preece (2005) both found that unreliability of service (e.g., having a server

go down) was among the most frustrating occurrences. Frequent breakdowns likely lead to a lack of perceived competence (and thus trust) in the community infrastructure.

Perceived goodwill is also especially important in OMSCs. Wright's survey found that off-topic discussion and flaming were associated with lower perceived emotional 
support (2002). Maloney-Krichmar and Preece suggest that low levels of hostile behavior and high levels of helping behaviors contribute to members' "ability to develop a sense of trust toward the online community” (2005, p. 219). In order to bolster perceived goodwill (and thus trust) in OMSCs, several authors encourage diligent moderation and tools that help moderators (Leimeister \& Krcmar, 2005; Meier et al., 2007; Wright, 2002), while others encourage strong group norms of support and reciprocity (Maloney-Krichmar \& Preece, 2005). Leimeister \& Krcmar also encourage community transparency, welldefined privacy policies, and clarification of community roles (2005).

\section{Supporting Conversation Reuse}

Many OMSCs and community designers have recognized the importance of supporting some form of reuse, so that people who are not actively participating in the immediate conversation can still benefit from it. For example, the ACOR organization was initially started in order to capture and make available the email messages from cancer-related OMSCs (Ferguson, 2007). Nearly all OMSCs discussed in the research literature include email message archives or prior forum posts, although they may only be available to registered members. Some communities strongly encourage new members to read the archive before posting, while others do not.

As mentioned previously, Preece mentioned the potential information sharing benefits of FAQs and recommender systems, but warns that they may disrupt the emotional support activities (1999). Instead, she suggests more conservative approaches such as encouraging people to select meaningful message headers and allowing personal book-marking of bulletin board messages (Preece, 1999). Despite her warning against FAQs, there are many OMSCs that include FAQs or flagged forum messages that administrators encourage newcomers to read before posting. This variation in community policies suggests that there are multiple ways of balancing information sharing and emotional support and that some communities may lean more towards one than the other. 
Most studies assume that the benefits of reuse are primarily related to informational support (i.e., learning more about the medical topic) (e.g., Finn \& Lavitt, 1994; Preece, 1999; Wright, 2000). However, Galegher et al. suggest that members who only read messages (without contributing) may receive some emotional support as well (1998). Specifically, they argue that individuals who read messages from others struggling with similar issues benefit from a feeling that they are "not alone” (Galegher et al., 1998). This sense of validation, especially of negative feelings, is typically supported better by peers with related experiences than existing social networks without similar experiences (Helgeson \& Cohen, 1996). The extent to which members can feel validated by simply reading other's experiences remains to be shown, but it seems reasonable that some benefits are possible.

Galegher et al. also speculate that when "a reader reads advice couched in the terms of informal, person-to-person speech, the reader is likely to take the advice personally because there is no other 'you' physically present to whom the advice could refer” (1998). Thus, when members say things like “Hang in there. You are not alone” or "We are here for you" each reader can read it as a personal suggestion to them (Galegher et al., 1998). This may increase the likelihood that they will take it to heart. Thus, when individuals reuse others’ conversations, they may receive social support benefits.

It is also possible that readers benefit from the positive emotional modeling of others, especially since several studies found that there is a significant amount of positive emotion expressed in OMSCs (e.g., Fernsler \& Manchester, 1997). Maloney-Krichmar and Preece even suggest that filters could display only encouraging messages when desired (2005).

Finally, it is possible that medical practitioners can benefit by reusing content from OMSCs. Dan Hoch describes the many benefits that he received from reading messages from OMSCs related to epilepsy, despite the fact that he is a highly trained medical expert in that area (Hoch \& Ferguson, 2005). These include knowledge of 
practical tips on living with epilepsy and social issues that arise because of the disease, as well as knowledge that many patients want to know the details of their disease and are fully capable of understanding them (Hoch \& Ferguson, 2005). Prior to his participation in the OMSC, he did not provide this type of information to patients (as is typical of medical practitioners in general). However, after his participation, he recognized the value of sharing this practical information with patients, as well as introducing them to the online community.

Other researchers have found the use of illness narratives to be useful in educating doctors and encouraging them to be more empathetic and responsive to the needs of their patients (Charon, 2001). As mentioned previously, many personal experiences are shared in OMSCs. The high opportunity cost of medical practitioners' time makes it unlikely that they will read through entire discussion archives. However, communities could be encouraged to create their own illness narratives, lists of "things I wish my doctor knew," and other distilled community insights. Even if medical practitioners won't read them themselves, members could bring them to their doctors or use them to help frame their conversations. Indeed, many members already use OMSCs to prepare for doctor's visits (Ferguson, 2007).

\section{Discussion}

Online Medical Support Communities are an important and unique resource for individuals dealing with medical concerns, as well as their caretakers. They are particularly helpful at sharing practical medical knowledge in a highly personalized way. Their free, around-the-clock availability from home and rural areas make them a viable option for receiving answers to questions when other options are unavailable or too costly. They provide access to empathetic peers, even for the rarest conditions. And the possibility of remaining anonymous and hidden in a world of text allows people to ask questions and share personal experiences of a sensitive nature and not be pre-judged by 
visible cues. Misinformation is shared within these communities of laypersons, but is often corrected by other members or brought to a doctor's attention. Their virtual nature does not allow some desirable forms of support (e.g., giving a hug), but has allowed strong social bonds to form nonetheless, bonds that have often led to offline relationships. Although their positive effect on medical and psychological outcomes have not yet been systematically demonstrated, numerous studies have consistently shown that members self-report significant positive impacts, while negative impacts are rarely if ever mentioned.

The vast majority of OMSC studies have been descriptive in nature. They have highlighted the activities and values important to these communities. These include activities similar to those occurring in other online support communities such as information sharing through question and answers, and social maintenance activities such as keeping the discussion on-topic and enforcing group norms. They also include a greater abundance of certain activities such as providing emotional support and sharing personal narratives. Like other online support communities, question and answering occurs in a highly contextualized and personalized manner. Unlike some online support communities, most members answer questions and provide advice through sharing their own personal experiences. Even practical question and answer threads are littered with encouragement and expressions of empathy.

While there are some important differences between OMSCs and css-d, many of the core activities and values are similar, making it likely that many of the best practices gleaned from css-d will apply to OMSCs. For example, the prevalence of highly contextualized question and answer discussion, the emphasis on supporting newcomers and keeping the conversation friendly, the strong social ties among core members, and participation patterns are all similar. Although it is possible that the inclusion of a wiki (like an FAQ) could upset the information/emotional support balance (Preece, 1999), a wiki repository is a much more flexible tool, allowing the community to use it however it 
deems appropriate. The wiki repository does not necessarily have to be restricted to hard facts; it can include information of many genres such as personal profiles that have been used to help facilitate emotional support. More generally, the fact that medical information and social support are mixed together in community archives suggests that there may be an even greater need for some type of distillation for those interested primarily in the information content.

However, it is possible that some differences will make insights gleaned from cssd less applicable to OMSCs. For example, the importance of quality information (often associated with certified medical experts) and the difficulty of validating information may discourage "patients" from sharing their ideas in more long-lasting and "official” venues (such as a wiki repository). However, this hesitation may encourage them to cite expert sources or qualify their own experiences so others don't blindly follow them practices already commonly performed in some OMSC discussions (Galegher et al., 1998; Wikgren, 2003). The lack of technical expertise among OMSC members (as compared to css-d members) could be a problem in contributing to a wiki repository. This suggests that documentation on how to use the software and personal demonstrations may be needed in OMSCs where they were not at css-d.

In summary, OMSCs can likely benefit from the introduction of a wiki repository, although motivating participants to contribute may be more challenging than it was at css-d. In addition, the wiki repository may be used for new purposes such as facilitating emotional support. Some authors have suggested that personal profile pages may be particularly useful in this context, but there may be other wiki genres that also facilitate emotional support. Because the wiki is such a flexible medium, it is likely that OMSCs will tailor it to meet their own unique purposes. This would be consistent with the way communities have tailored the comparatively simple threaded conversation medium to serve a surprisingly rich array of purposes. 


\section{CHAPTER 9 \\ MEDSHELF - INTRODUCING A WIKI REPOSITORY}

In this chapter, I introduce an action research project, where existing online medical support communities (OMSCs) were recruited and provided with a customized wiki to use as a community repository. They were also provided with training materials (see Appendix 3) and occasional advice intended to help them use the wiki effectively. The wikis (and training materials) are found at Medshelf.org ("MedShelf," 2007). The advice included in the training materials is based on the best practices identified in the css-d study (and summarized in Chapter 7), along with an understanding of the differences between technical and medical support communities as outlined in Chapter 8 .

This action research project is important for several reasons. First, it helps validate the findings from the css-d study. It also helps to characterize the ways in which context is important in their application, since it applies them in a medical support context instead of a technical support context. I have also used the action research recruitment process to better understand the issues important to communities when deciding whether or not to implement a repository. This information, coupled with the experiences of the 3 communities with which I have worked have helped characterize the types of communities that are good (or poor) candidates for using a wiki repository.

In this chapter I begin by describing the primary research questions and methods used to explore them. Next, I introduce the participating communities and the Medshelf website. I then present findings in two sections ('Considerations when Deciding to Adopt a Wiki Repository' and 'Successfully Introducing a Wiki Repository') and discuss their implications. Below is an outline of the sections:

- Research Questions \& Methods 
- Communities

- Medshelf.org

- Results and Discussion

o Considerations when Deciding to Adopt a Wiki Repository

o Successfully Introducing a Wiki Repository

- Lessons Learned

\section{Research Questions \& Methods}

\section{Research Goals and Questions}

The ultimate goal of the study was to learn how to improve online support communities through the introduction and use of wiki repositories. Although some online support communities, such as css-d, have successfully integrated a wiki repository with their threaded conversations, many have not. Some communities may not be good candidates for wiki repositories. For example, they may be too small or inactive to garner enough labor to maintain one. Other communities may be good candidates, but have never considered it as a possibility or have prematurely rejected the possibility. Still other communities may have considered it (and even tried it), but not know how to successfully introduce a wiki. Unfortunately, no existing research helps inform a community that is considering implementing a wiki repository to know where it falls within this spectrum. Likewise, if a community is a good candidate, little research helps it know how to successfully introduce a wiki repository. This study is a first step in addressing these issues and specifically addresses the following primary research questions:

1. What are the key factors that communities should consider when deciding to adopt a wiki repository? What do these factors imply about the types of communities that are good (or poor) candidates for a wiki repository? 
2. How can existing online support communities effectively introduce a wiki repository (i.e., what social practices, community roles, activities, and technological features are important)?

Future research based on this action research project will address challenges that arise with using wiki repositories for extended periods of time. Future research will also examine which wiki genres are particularly important to OMSCs, as opposed to other online support communities.

\section{Why OMSCs?}

To address these questions I recruited a few OMSCs to participate in the action research project. Selecting only medical support communities was practical because it allowed us to create recruitment and training materials that were customized for this subset of communities. It was also useful from a methodological perspective because it helped reduce the amount of variation across the communities that we studied, making comparison across groups more useful. OMSCs were chosen for the following reasons:

- They are prevalent and thus offer a large pool of potential communities, as well as a large audience for the research findings and suggestions.

- They have many potential barriers to implementing a wiki repository. For example, concerns about privacy, a lack of technical expertise among members, the potential for harm from poor quality content, and the prevalence of social support (in addition to knowledge sharing) all raise potential barriers to implementing and benefiting from a wiki repository. These concerns are common to many other types of online support communities as well, although often to a lesser extent.

- They have been studied more than any other type of online support community. This prior research base provides insights into the issues that will be important and the activities that are prevalent among OMSCs (as outlined in Chapter 8). This knowledge was helpful in tailoring the training materials and suggestions for the communities and knowing what issues to explore.

- They have the potential to significantly improve the well-being of individuals, and thus are an important subset to study even if some results do not generalize to other types of support communities. 


\section{Action Research}

I chose to use an action research approach to address these research questions. The term “action research” has been used by many researchers in different, but related ways and may relate to a general class of methods or a specific sub-class of those methods (Baskerville, 1999). Here, I use it in the broad sense, as introduced to information systems researchers (Baskerville, 1999) and computer scientists (Avison, Lau, Myers, \& Nielson, 1999). In this sense, action research is a collaborative endeavor between researchers and subjects, where change experiments are performed and their effects are studied in a mutually acceptable ethical framework. Like most other qualitative research, action research "aims for an understanding of a complex human process rather than prescribing a universal social law” (Baskerville, 1999).

The goals of an action research project are twofold: to benefit the research subjects and to learn from the experience in a way that informs further research and theory. Thus, the aims of the researchers and subjects must be in line with one another. In this sense, action research is a close sibling to "participatory design” and related methods (Avison et al., 1999). It differs in that it tests and prescribes various forms of social action, as well as technical designs, whereas most participatory design studies are primarily focused only on the design of a technical artifact.

Action research is particularly well suited to address certain research questions including:

- Questions that are practical in nature, such as questions related to system design (Avison et al., 1999; Baskerville \& Myers, 2004).

- Questions that occur in complex social settings where there are many variables and components that may be difficult or impossible to characterize. In such situations it is likely that more will be learned from actively intervening and observing the results than predicting the effects of interventions by other means (Baskerville, 1999). 
- Questions that relate to change processes, such as introducing a new software system to an organization (Baskerville, 1999).

- Questions that relate to phenomena not yet occurring naturally.

All of these factors were pertinent to this study. Thus, an action research approach allowed me to test and develop practical suggestions about the change processes related to implementing a community repository into a complex social setting. Furthermore, because wiki repositories are not currently used by OMSCs, it was not possible to empirically study them in this important context without first introducing them. It was also ethically responsible to continually work with the groups to assure that no harm came to them and that the project succeeded as best it could.

\section{Research Procedures}

In action research it is particularly important to describe the research procedures followed because of the influence the procedures can have on the outcomes. As is typical in action research studies, I took an ongoing active role in introducing the wiki repositories to the communities I have worked with. Throughout the project I documented my own involvement to better understand which actions were initiated by myself and which were initiated by others. In this sense, I have treated myself as a subject of the study and treated my communication with the other subjects as data.

Treating myself as a subject helped me clarify my own role in the project and the impact of my suggestions and actions on the community. However, my suggestions are all somewhat tainted in the sense that I play a unique role (as researcher) that is not likely to exist in other communities. For this reason, I tried to take a backseat role and only intervene when I was asked or when I noticed the project was failing. However, when called upon, I tried to be as responsive and helpful as possible, often sharing my experiences and opinions based on my work with other communities. Most of these interactions were with the community members tasked with rolling out the wiki. As a 
result, most community members only saw the actions of their community leaders and peers, even though I suggested some of those actions.

My involvement included several specific activities. I began by recruiting communities to participate in the study. I encouraged communities that were considering participating in the study to speak with me by phone so that I could explain the project and answer questions directly. I also oversaw the creation of the Medshelf.org website, which is based on MediaWiki, but includes some customization intended to make it more user friendly. Most importantly, it includes suggestions for community leaders about how to introduce the wiki to the community and promote its use (see Appendix 3). It also includes customized help pages that describe how to use the wiki. Even with these pages, community leaders contacted me via email many times to help address their specific questions. I typically reiterated suggestions that I had made in the recommendation pages when providing input. I also occasionally asked for updates on the project and asked if the community leaders had any questions or concerns. This was typically done when I noticed a lag in communication and wiki editing activity and often led to follow up discussions about issues that had arisen.

\section{Data}

Both qualitative and quantitative data were collected throughout the project. This data came from the process of recruiting communities, as well as from the 3 communities with which I worked: The GIST support, Graves’ disease, and Pain management communities (described in the following section). The data sources are discussed below:

\section{Community Messages}

Each of the 3 communities I worked with allowed me access to prior messages stored in an email archive or bulletin board threads. Header information for a recent 2month period from each community was downloaded for quantitative analysis. In 
addition, the bulletin board software used by two of the communities included information on how many times each message was viewed, as well as some overall participation statistics for the entire duration of the board.

\section{Wiki Content}

In addition to the actual wiki page content, the MediaWiki database ("MediaWiki," n.d.) provides information on who makes wiki edits (including a username if the person is logged in and an IP address if they are not), how many bytes were changed, when the edits were made, and various page statistics (e.g., number of edits, page size, how many pages link to it).

\section{Webpage statistics}

Log data has been tracked for each community wiki separately. The Google Analytics tracking features have been used to gather information on the websites that others come from, search terms used to arrive at the sites, and pages that have been visited ("Google Analytics," n.d.).

\section{Interviews}

Six interviews were conducted with members of the three groups to help understand the experiences and perceptions of the wiki by members. Questions related to the barriers encountered when introducing the wiki, the usefulness (or lack of usefulness) of the training materials and technology, the motivation of the individual, and general perceptions of the project. Five of the interviews were conducted via phone and transcribed and one was conducted via email. The main wiki advocate (and list moderator) from each of the 3 communities was interviewed, as well as two active wiki contributors, and one non-contributing moderator who encouraged others to participate. 
Personal communication

All correspondence between myself and community members (typically those who headed up the wiki project) was saved and analyzed. These included over 200 messages. In addition, I asked moderators to copy me in on private email messages sent to other community members so that I would be aware of them. The GIST support community did this frequently, while the others did so only occasionally. In addition, I spoke with community leaders infrequently to describe the project, offer advice, and help them with using the wiki. Brief summaries of these conversations were written immediately after the conversations. In addition to my personal communication with the communities we ended up working with, I also collected all responses and phone conversations with communities that we did not end up working with.

\section{Email questionnaire}

An email questionnaire was sent to the GIST support and Graves’ disease communities that asked questions about use and perceptions of the wiki. Three members from the GIST cancer group and one member of the Graves' disease group responded to the questionnaire. Three of the 4 respondents had contributed at least once to the wiki, and none of them were part of the initial "wiki team.” The one who had not contributed to the wiki was one of the moderators of the GIST cancer community and had promoted the wiki in email messages. None of these respondents were among those interviewed.

\section{Analysis}

Both quantitative and qualitative methods were used to analyze the data. Quantitative analysis included analysis of participation patterns in the community threaded conversations, as well as the wiki. When possible, I tabulated statistics comparable to those used in the css-d community to allow for direct comparison. Because 
I hosted the wiki, I was able to capture better data on wiki participation patterns and website access logs than was available with the css-d community. As in the css-d study, qualitative analysis was performed in the grounded theory tradition where the major themes were derived from the data rather than pre-defined ones (see Chapter 3 for a discussion of how I used these approaches in the study of css-d). Interview data, community posts, questionnaire comments, wiki content, and personal communication were all grouped into preliminary categories, which were later refined through an iterative process. The themes most salient to the communities and directly related to the research questions are presented in this paper.

\section{Communities}

\section{Medshelf Recruitment Experience}

The goal of the recruiting effort was to attract a handful of communities that desired to augment their threaded conversation with a community wiki. Active communities that fit the definition of OMSC provided in Chapter 8 were invited. I hoped to attract communities with different characteristics (e.g., number of members, software platform, medical topics) in order to see how their experiences varied. A random sample of communities was not needed, since the focus of the study was not to generalize findings to all OMSCs. However, a systematic approach to recruiting communities was used in order to learn from the experience of recruiting online communities for an actionresearch project.

Communities were recruited to participate in the study via email. A standard recruitment email (see Appendix 4) was sent to the community administrator(s) explaining the project and some of the potential benefits of participation. Initial recruiting began in the Fall of 2006 (Sept-October). This initial recruiting effort attracted two potential communities: the GIST support community (which continued to participate) and 
another community that stopped participating after we set up the initial wiki for them. After learning from the GIST support community's initial experience, I continued recruiting communities in the early months of 2007 (Jan-March) using an updated recruitment email (also see Appendix 4). This resulted in the recruitment of the Graves' disease and Pain management communities.

A few different sources were used to identify communities of potential interest. These included the following 4 sources:

- CataList, a master list of communities using the LISTSERV® email list software ("Listserv, L-Soft International," n.d.). OMSCs were identified by the community names and descriptions. Only those that included over 100 members and showed regular activity in the recent past were included. OMSCs associated with ACOR.org were not recruited, since many of them are participating in a different study (Meier et al., 2007).

- Yahoo! Groups listed in the "Health \& Wellness > Support" category ("Yahoo! Health Groups," n.d.). OMSCs were identified that had over 400 members and had sent at least 21 messages in the past 7 days. A subset of these (28 out of 42) were contacted. The other communities were not invited because it became apparent that this source was not producing many leads (see Table 9.1).

- Personal referrals. I also used a convenience sample by asking my friends and the University of Michigan's School of Information students to recommend any OMSCs that they knew of who may be interested in participating. This resulted in 4 communities being invited. In addition, 3 communities were referred to me by other communities that I had contacted about the project.

- Websites. A handful of communities were invited because I found their communities through a Google search. Active OMSCs with many members and a high Google ranking were invited.

In total, 4 of the 74 communities (5\%) that received an email invitation had us set up a wiki for them, although one of them stopped participating once we had set it up (for an unknown reason). It is hard to know if this number is high or low, as no comparable studies were identified. Our recruiting method resulted in only 3 failed emails ( $4 \%$ of all 77 sent emails). However, some recruitment email messages were likely not read since they may have been caught in spam filters or sent to email accounts that are not regularly 
monitored. In total, 15 of the 74 contacted communities (20\%) replied to the initial email invitation. Of these, 5 were brief responses declining the offer. Six of them led to extended conversations about the project but no wiki, and 4 led to us setting up a wiki for the community. These results are summarized by source in Table 9.1.

Table 9.1: Medshelf Recruitment by Source

\begin{tabular}{|c|c|c|c|c|c|}
\hline Source & No response & Brief response & $\begin{array}{l}\text { Extended } \\
\text { Conversation }\end{array}$ & $\begin{array}{l}\text { Set up } \\
\text { wiki }\end{array}$ & Total \\
\hline CataList & 28 & 3 & 3 & 2 & 36 \\
\hline Yahoo! Group & 27 & 1 & & & 28 \\
\hline Referral & 4 & & 1 & 2 & 7 \\
\hline Website & & 1 & 2 & & 3 \\
\hline Total & 59 & 5 & 6 & 4 & 74 \\
\hline CataList & $78 \%$ & $8 \%$ & $8 \%$ & $6 \%$ & 36 \\
\hline Yahoo! Group & $96 \%$ & $4 \%$ & & & 28 \\
\hline Referral & $57 \%$ & & $14 \%$ & $29 \%$ & 7 \\
\hline Website & & $33 \%$ & $67 \%$ & & 3 \\
\hline Total & $80 \%$ & $7 \%$ & $8 \%$ & $5 \%$ & 74 \\
\hline
\end{tabular}

The most successful method of recruitment was referrals. This was likely the case because the referrers only recommend groups that they believe would benefit from the experience. Importantly, the two communities that had us set up a wiki were referred from a list owner of a website that we had contacted. This suggests that initial recruitment messages should ask community administrators to recommend other communities that may be interested, even if they themselves are not. The websites that were contacted also led to a high level of response and consideration. This may be because a specific administrator name and email address was provided, which was used in the communication. This was different for the CataList and Yahoo! Groups communities where a standard administrator email address is used (e.g., CommunityNamerequests@CommunityUrl.org) and names of administrators were not available. Finally, Yahoo! Groups were less likely to respond than CataList groups. This may have to do with the way that he administrator emails are processed or used. For example, it is possible that the Yahoo spam filter caught out message or that CataList groups often use 
the generic administrator email address, while Yahoo! Groups do not by tradition. It is also possible that the updated recruitment email was not as effective as the original (since the original was sent to the CataList groups and the updated one sent to the Yahoo! Groups) (see Appendix 4). However, this is unlikely since it was used to contact the websites and some of the referrals, which were successful.

Later sections discuss some of the reasons communities were worried about participating, as well as the reasons they wanted to participate. Next, we describe the 3 communities that used the Medshelf wiki repository.

\section{GIST Support International (GSI)}

GIST Support International (GSI) is an active OMSC and non-profit organization for individuals affected by Gastrointestinal Stromal Tumor, a rare type of cancer. Their mission statement, vision, and guiding principles make it clear that they are a patientcentric community that "reaches out to GIST patients and their families and friends to provide education and support” as well as "promotes and encourages ongoing research in the quest for a cure for Gastrointestinal Stromal Tumor” ("GIST Support International,") They were formally organized in 2002, although many of their members knew each other from other related OMSCs.

GSI is an all-volunteer organization with members that serve on various committees including the operating committee, greeters, phone pals, science committee, website committee, and pediatric committee. Their primary activities include running an email list (using Listserv software) and maintaining a website with extensive information on new research and patient education materials. They have also been involved with creating a television documentary recently. In 2006 they had an operating budget of just under $\$ 200,000$ provided by various grants and a few personal donations. It was spent mostly on a television documentary and website content creation and maintenance 
("GIST Support International - Organization,"). The majority of dollars spent in prior years have gone to the website.

GSI members use LISTSERV® email list software as their primary means of communication ("Listserv, L-Soft International," n.d.). It allows members to send and receive messages sent to a single list community email address. Since September of 2003 all list messages are made accessible to registered users through a searchable and browseable archive. The software also provides administrative functions such as the ability to add and delete members. Unlike css-d, the software is configured so that replies to list messages are sent to the entire list by default, not directly to the sender.

In June 22, 2007 there were just over 856 subscribed members. In April and May of 2007 a total of 236 members posted 1,600 messages, representing an average of 26 messages a day. There is an average of just under 3 messages per thread, a number comparable to the css-d community. As in other online support communities, participation is skewed with $50 \%$ of the content being provided by only $7 \%$ of the posters, and the top poster accounting for $8 \%$ of all posts. In addition, there are many lurkers who subscribed but never post. As is also common, the administrators are among the most active members. The total number of messages per month has remained relatively stable, although it has declined some since the earliest time period for which I have data (late 2003 and early 2004).

GSI members participate in all of the typical OMSC activities described in Chapter 8. A casual reading of all messages sent in April 2007 indicated that most were social support, but information sharing was common and examples of announcements and administrative messages were found. Specific topics include discussions of treatments, medication side effects, doctors, insurance, cancer-related events, clinical trials, and research articles. Members frequently post updates and share their recent experiences with the disease, medications, and interactions with medical professionals. Because of the serious nature of the disease, two announcements of death and a 
significant number of condolence messages were sent to surviving family members. It is clear that many strong relationships exist within the community, as evidenced by frequent references to communication happening outside of the list and regularly scheduled faceto-face meetings organized by the group. These meeting occur at research presentations, as well as members' homes for members who live in surrounding areas.

\section{MediBoard Groups}

Two of the OMSCs participating in the MedShelf study are associated with the MediBoards Network, a for profit company that hosts medical support forums ("The MediBoard Network,"). The MediBoards Network is funded by advertising dollars, which accounts for the numerous ads found throughout the site.

The forums are currently using IP.Board software, a top-of-the-line commercial bulletin board product ("IP.Board, IPS Community," n.d.). They moved over to this platform on April $5^{\text {th }} 2007$ after using a different product since 1998. IP.Board includes the ability to post messages in a threaded format, customize personal profiles, use basic formatting (e.g., smileyfaces, emoticons, bold, italics), display "hot” (i.e., active) threads, send personal messages to other members, and perform administrative functions (e.g., ending a thread, closing a user's account). All prior messages are retained in the forum and can be searched or browsed through. Threads with the most recent message are shown in reverse-chronological order. In addition, some threads have been "pinned" to the top of the forums because they contain information of lasting value.

Data for the MediBoard forums was collected on June 22, 2007. It includes 229,610 messages spread across the 6 forums, as well as information from the personal profiles of the 4,247 registered members. Only two of the forums are participating in the

study, but they account for $99.7 \%$ of all MediBoard posts. They are Graves’ Disease and Thyroid Conditions community (Graves Community) and the Chronic Pain / Pain Management community (Pain Community). A subset of messages for the most recent 60 
days was analyzed from each of these communities to indicate current levels of participation. They were also read in order to gain a better understanding of the activities and themes important to the communities. Below is a description of each of the communities.

Graves’ Disease and Thyroid Conditions community (Graves Community)

The Graves Community is an active, thriving OMSC. It accounts for $61 \%$ of all MediBoard messages. An average of 37 messages was sent each day during the most recent 60 days. In total, the forum includes 139,873 messages found in 16,711 threads (an average of 8.4 messages per thread). During the past 60 days, each thread was read an average of 115 times (including multiple views by the same individuals).

The membership participation information is provided for the entire history of MediBoard. As of June 22, there were 4,147 registered MediBoard users. In addition, there are "Guests" who view posts but do not register. The number of Guests visiting the site at any given time is shown, although it is not tracked over time, making it impossible to estimate. However, most of the times I have visited the site in the past few months there have been a handful of Guests viewing posts. As expected, participation patterns in the Graves Community are skewed with only 25 members (1\% of all members) accounting for $50 \%$ of posts and the top poster accounting for $9 \%$ of all posts.

There are three volunteer moderators of the Graves Community, although 1 of them is not currently active. The two active moderators are the most frequent posters on the list, accounting for almost 13\% of all Graves Community messages. They perform typical moderator activities such as clarifying policies, motivating participation, and discouraging flaming. Off-topic conversation is generally allowed and moderators even participate in it. It is typically either labeled as such in the thread headers (e.g., "Off Topic--Digital camera question”), or mixed in with on-topic conversation. In fact, several of the longest threads were off-topic, suggesting they are acceptable within the 
community. These posts discussed things like bird watching, television shows, and the meaning of login names. It is clear from these discussions that members have developed friendships with one another that carry over to other aspects of their lives and using the list for off-topic conversation is a way of strengthening those friendships. These friendships continue outside of the list through phone calls and even regular face-to-face get togethers for members in a similar region of the country.

Graves Community members participate in all of the typical OMSC activities described in Chapter 8. Because of the nature of Graves' disease, much of the discussion revolves around understanding and interpreting blood tests. Many members’ signatures include results from these blood tests and norms exist around how to properly share the test results with the group. Members also discuss treatment options, side effects of medications and surgery, coping strategies, and provide social support to one another. This social support is especially common since high anxiety, irritability, and depression are all associated with Graves’ disease.

A number of threads have been "pinned” to the top of the forum, so that they are always visible. As would be expected, these threads are read more often (an average of 158 times per thread) than typical threads (e.g., threads in the past 60 days were read an average of 115 times). The thread “Our Personal Graves Disease Stories” is the most viewed thread with 403 visits followed by the "Research Articles and Research Links" thread with 260 visits. Other threads are community related, such as the "Pictures of Us" or related to administration of the list, such as "How to post your labs" and "No personal attacks.”

In addition to the discussion forum, the Graves Community administrators have also created a "Thyroid 101” forum, which includes hand-selected messages intended to educate newcomers. New content can only be added by the administrators. On June 22, 2007 it included 24 threads with 34 replies. Threads within the Thyroid 101 forum were visited an average of 112 times, about the same as the new threads from the past 60 days. 
The content in this forum, as well as many of the pinned items from the discussion forum demonstrated the need of the Graves Community to create resources for long-term use. Many of these materials were later added to the wiki, when it was initially seeded.

\section{Chronic Pain / Pain Management community (Pain Community)}

The Pain Community is currently less active than the other two communities. It accounts for 39\% of all MediBoard messages historically, but is currently much less active with only 5 messages sent on average per day during the last 60 days. In total, the forum includes 89,101 messages found in 10,692 threads (an average of 8.3 messages per thread). During the past 60 days, each thread was read an average of 36 times.

As with the other communities, participation patterns are highly skewed. Only 12 members ( $1 \%$ of all members) account for $50 \%$ of all messages sent to the forum since its inception. The top poster accounted for $14 \%$ of all messages. The sole community administrator is the second most active member and accounts for $6 \%$ of all messages.

Administration of the list is similar to the Graves Community. In general, intervention by the administrator is rare and off-topic discussion is allowed and common among members. Much of the conversation seems to revolve around providing updates on things unrelated to chronic pain such as the weather, vacations, health issues of family members, and experiences at work. The overall tone is reminiscent of a group of longtime friends that keep each other up-to-date on their lives. It is clear that several members have developed close friendships that persist outside of the forum. Members have even created a map that shows where members live.

In addition to the updates and off-topic discussion, there is some information sharing and social support. Members ask questions about insurance, medications, and alternative therapies intended to help reduce pain. As is common in OMSCs, the majority of answers to these questions are based upon personal experience. However, members 
also announce research articles and pertinent news (e.g., the release of a new pain-related drug).

Like the Graves Community, the Pain Community also has messages “pinned” to the top of the forum. These include a welcome message to newcomers, an announcement of the wiki, a birthday list, and information on where people live (with a link to the map showing their locations). These messages show the importance of the social relationships within the community, which overshadow the information sharing that occurs within the community.

\section{Medshelf.org}

The Medshelf.org website is the home of the community wiki repositories. It includes a technical infrastructure for collaborative authoring as well as content designed to help communities effectively implement and use the wikis. A separate wiki was set up for each of the 3 communities participating in the study and is accessible as a sub-domain of the medshelf.org website. This allows each community to have complete control over every aspect of its wiki. We also changed the color scheme and included a logo (suggested by community leaders) for each of the community wikis to help differentiate and personalize them. The technical infrastructure and content that was provided on each of these wikis is described below.

\section{Technical Infrastructure}

Medshelf.org is based on the MediaWiki version 1.9.2 open source software ("MediaWiki," n.d.), which is used to run many wikis, including Wikipedia. The software includes all of the basic wiki features (see Chapter 5 for a description), as well as some advanced features. Advanced features include the ability to assign different user roles with associated actions (e.g., “administrators” also called "sysops” can delete pages and protect pages from being edited), receive updates when pages are edited, revert prior 
edits, insert images, and easily link to Pubmed articles and books by using the PMID or ISBN numbers respectively.

A customized skin was developed in order to emphasize the most common functions (e.g., Edit) and pages (e.g., Main Page, Recent changes, and All pages). A screenshot of the Main Page is shown in Figure 7.1 showing what the page looks like to an administrator who is logged in (using the Graves wiki as an example). When not logged in, only the 'Edit' and 'History' buttons are displayed, along with a single 'Log in / create account' link at the top. When non-administrators are logged in it looks similar to Figure 7.1 except that the 'Protect' and 'Delete' buttons are not shown, as only administrators have the ability to perform those actions.

\section{Figure 9.1: Medshelf Main Page (Graves’ Disease Wiki)}

Graves Disease and Hyperthyroidism Wiki
Main Page | Recent changes | All pages | Upload image
Main Page
Welcome to the Graves' Disease and Hyperthyroidism Wiki! This wiki serves as the community's long-term memory and public outlet
to the world. Please read our About page and Disclaimer and understand that the information found in this wiki is not provided by
medical experts. We hope that you find the content useful and help improve it by making your own edits (see the Wiki 101 page for
basic instructions). After all, it is a community project run by individuals like you!
Graves' Disease and Hyperthyroidism Forum
Gratchlist | My contributions | Log out
Come visit our Community Forum to discuss Graves' disease and other hyperthyroid conditions. Make friends with other Graves'
disease patients, give and share tips on coping. It is an active community with several posts a day.

Other major changes to the standard MediaWiki interface included the removal of "talk" pages associated with each wiki page and the customization of special pages (e.g., the search page) to remove unnecessary information (e.g., links to talk pages and advanced namespaces such as the 'MediaWiki' and 'Template' namespaces). Minor changes included the renaming of some functions (e.g., "Moving” a page is now called “Renaming” a page).

\section{Content}


Each community wiki included pre-populated wiki pages intended to help the communities get started using the wiki (see Appendix 3 for some of the more important pages). In total, 81 pages were provided. These include 51 help pages describing how to use various features of the software (e.g., 'Editing,' 'Watching pages,' 'Starting a new page'). The other 30 pages include content on social practices related to the wiki. These include suggestions on how to get started (e.g., 'Getting started'), descriptions of roles people might play (e.g., 'Wiki helpers’), genres that might be helpful (e.g., 'Links Genre'), messages introducing the wiki to the email list ('Sample Roll Out Email'), and sample content (e.g., 'Sample Main Page). It also includes pages related to the site as a whole such as the 'About,' 'Privacy policy,' 'Medical and Legal Disclaimer,' and ‘Copyrights’ pages.

When communities began the project, the main contact person from the community (in each case this was one of the moderators of the list) was encouraged to read the 'Getting Started' or 'Simple Start' page, both of which provided the major suggestions on how to effectively get the wiki started. These pages are not easily accessible to all members (because they are in the "Project" namespace) and are intended for the individual or handful of people involved with getting the project started. The specific recommendations were based on the css-d study best practices (outlined in Chapter 7), but customized to meet the needs of OMSCs. The outline of the 'Getting Started' page is included below, with a full copy of the page (and many associated pages) available in Appendix 3.

'Getting Started' Table of Contents

1. Announce the Wiki to Your Community

2. Establish a Wiki Team

2.1 Create the Wiki Team

2.2 Determine Member Roles

3. Decisions

4. Seed the Wiki with Content 
4.1 Systematically Decide What Should be Included

4.2 Create Good Content in a Useful Format

4.3 Organize Your Content

4.4 Update Existing Pages

5. Promote the Wiki

5.1 Initial Activities

5.2 Ongoing Activities

Another important page provided to communities was the 'Wiki Guide,' which is the starting point for regular community members (see Appendix 3). It includes a description of what a wiki is, how it relates to the email list (or bulletin board), suggestions on how members can contribute, things to consider before contributing (e.g., privacy), and suggestions on how to learn how to use the wiki including links to the most important help pages.

\section{Results}

\section{Considerations when deciding to adopt a wiki repository}

This section addresses the first research question outlined above: What are the key factors that communities should consider when deciding to adopt a wiki repository? And what do these factors imply about the types of communities that are good (or poor) candidates for a wiki repository?

The decision to adopt a community repository in the first place is an important

one. The recruitment experience described above provided several insights into the issues most salient to the OMSCs I contacted. Although I do not have data on the reasons why most communities failed to participate in the study, I do have data from several communities that ended up participating, as well as several that did not (see the 'Extended Conversation' and 'Set Up Wiki' columns of Table 9.1).

Perceived Barriers 


\section{Too Much Extra Work}

One of the major concerns voiced by the communities we tried to recruit was that the wiki repository would require too much extra work. For example, one community that seriously considered participating in the project decided not to by stating: "It's become clear that we don't have the time/people to take part in your project at this point. This has always been the case, but I sometimes forget.”

Another community moderator who was seriously considering the project declined in the end stating that it was "more than I can currently manage.” Importantly, another community administrator offered his opinion to the other moderators that the project would be "more work than it's worth for this listserv" particularly because it is not a very active list. Even communities that did participate in the study asked questions about the effort that would be required (e.g., "how much work is it to hunt down spam?”).

To help overcome this problem, I shortened and simplified the original 'Getting Started' page into the 'Simple Start' page. However, even this page included several suggestions and linked to many other pages that could be overwhelming at first. Although many of the pages proved helpful to communities that ended up participating (as discussed in the following section), some of the pages were never consulted (e.g., Wiki Genres), suggesting that there may have been too much information presented initially even after my attempt to reduce it.

Although it is not surprising that communities feared taking on the project because of the extra work, it does raise a few points worth considering. Unlike css-d, which created the wiki so that it would have a lower workload later (when its members answered frequently asked questions), the OMSCs we contacted did not see this as an obvious benefit. This was in spite of the fact that we mentioned that it could be used to help answer questions in our original recruitment email. This may be due to differences between technical and medical support groups. 
Another issue raised by the declining community above is that less active communities may be poorer candidates for wiki repositories than active ones. This may be true for two different reasons. First, the benefit of using the repository as an FAQ is less, as just discussed. And second, less active communities typically have fewer labor resources to draw upon, making additional projects more difficult to take on, even if there are potential long-term payoffs.

\section{$\underline{\text { Privacy Concerns }}$}

A few of the communities mentioned concerns about member privacy. One community described privacy at its "biggest concern." Some of the communities we contacted had closed email lists where members were screened before joining to assure that they were who they said they were. Other lists did not have any public archives because they were concerned about breaches of members' personal information. These communities were concerned about having a publicly accessible wiki. One community leader of a group of particularly vulnerable patients voiced his concerns about having a public wiki to the other community leaders in these words:

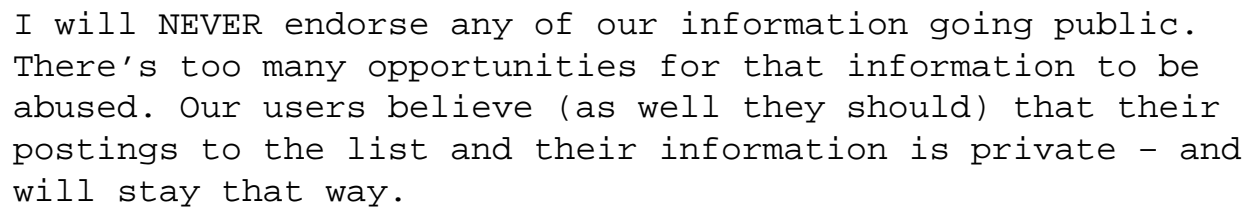

He later argued that educating the patients about what they should or should not post on the wiki would not be a feasible solution, especially given the lower mental acuity of the group members due to their medical condition.

The community leader of a different community voiced her concerns about privacy, while at the same time recognizing the potential benefits of having a publicly accessible wiki. Specifically, she liked the idea of helping share the knowledge from her group more broadly and potentially attracting new members to the group. In this way, the 
repository was seen as a possible middle-ground where members could post some information to the "outside world" while still having a private place in the email list to share personal information and support.

Although some communities were deeply concerned about privacy issues, other communities did not consider them a problem at all. In particular, the bulletin board communities that we work with never mentioned anything related to privacy. This is likely because all of their current participation is already happening in a publicly searchable location and they have adopted strategies to deal with that fact. For example, many members already self-monitor their posts to exclude any personally identifiable information.

This suggests that communities with serious privacy concerns may not be good candidates for a wiki repository. While it is possible to create a wiki repository only accessible to members, it requires the extra work of maintaining the list of members (since current software is not integrated well with email list software). It also loses some of its appeal and usefulness, notably for recruiting new members.

\section{Long-term Sustainability \& Data Control}

A few of the communities were concerned about the long-term sustainability of the wiki repository and issues about who controls the wiki content. This is likely at least partly due to the fact that it is a research project. However, one community leader was

attuned to these issues because of previous experiences with non-researchers, suggesting that these issues are important in other situations as well.

Two of the communities we spoke with (one of which ended up working on the project) asked if it would be possible for them to host the wiki on their own servers. They wanted to do this because they recognized that they would have control over the data and that it would not go away any time soon. However, they also recognized that it would require a significant investment in time and require expertise that they did not then have. 
In the end, the community we ended up working with decided to use our site at first and transfer the database over at a later time if necessary. Our decision to use an open source wiki (i.e., Mediawiki) that allows for exporting and importing of pages was important in convincing the community that this was a feasible approach.

\section{Technological Ability}

A couple of the communities shared concerns about the ability of their members to use the wiki technology. This was especially important for one group of members who the administrator described as "technologically challenged" due to their medical condition. This administrator also discussed how earlier attempts to have their members use chat rooms and create biography pages had failed. Another community leader mentioned that the original recruitment email was "over his head" technically and that he would need to take some time to sort it out. However, on the whole, this was not mentioned as a concern as often as the other concerns mentioned thus far. This may be because community leaders hadn’t yet tried the wiki and recognized its particular challenges, or it could be that community leaders are more technologically savvy than other members.

\section{Quality Control}

Three groups mentioned concerns about the quality of information given that anyone can edit the wiki. Inclusion of a disclaimer, stating that the content was provided by non-medical experts and that it should be verified by a trained professional was considered a good idea by two of the groups. In fact, several of the groups had disclaimers on their community websites already. One of the wiki team members of GSI asked about the process of submitting and reviewing wiki content. The following quote from an email message sent to the Wiki Team explains why access controls and peer review are important in this context: 
I think these are issues that have to be addressed at the onset. I would think there has to be some sort of quality control in place to prevent misinformation or inappropriate statements from appearing on the wiki. Unlike our standard GSI message board, where entries are read on a daily basis, deleted and usually never see the light of day again, this wiki will be used as a resource that will hopefully be accessed numerous times in the future. I think the content has to be concise, relevant and as accurate as possible.

Because the wiki content will presumably be read more often (as recognized by this author) and seen as more authoritative (as recognized by others), quality control mechanisms are more important than in the ongoing discussion. In response to this member's concerns, another Wiki Team member mentioned that pages will need to be monitored regularly and asked me to comment on the technical ways of doing this. I explained how people can use the "Recent Changes" page and monitor specific pages by "watching" them (i.e., subscribing to them). I also explained that it is technically possible to "protect" pages from non-administrator edits, but that this technique would probably lead to fewer edits in general. This explanation was acceptable to the community and it has not expressed concerns about the quality of information since this initial question arose. In this case, the technical ability of the software to "protect" pages and monitor changes was enough to dispel concerns, even though no pages have been protected as of early July 2007.

A different type of quality concern was raised by two different groups. They wanted to make sure that there was no commercial interest, as they felt that the project may be tainted if there was a corporate connection (e.g., with a pharmaceutical company). On the other hand, other communities were interested in the opportunity to use advertisements on the wiki in order to help fund their own community costs. This suggests that the funding source of the wiki is a significant consideration for community adoption of the wiki and one that is believed to influence the content.

Perceived Benefits 


\section{Broadening the Community’s Reach}

Several communities liked the idea of having a wiki repository to help broaden the community's reach to individuals who were not currently part of the community. In some cases the motive was altruistic. For example, one community leader explained to his group that if they participated in the study: "We will be able to allow others to know about how [community topic] can be done.” In other cases the main reason for extending the community's reach would be to help attract new members. This was the main reason the Pain Community wanted to participate, as it was looking for more active members. Another group (that did not end up participating) mentioned "allowing others who need support to find us and subscribe” as one of the main reasons it was interested in the project. As hinted at previously, this benefit can only happen if the wiki repository is made public - a practice that also raises the privacy concerns discussed earlier.

This finding suggests that communities that do not currently have a noticeable web presence may benefit significantly from a wiki repository. Many communities do not have the resources or technical expertise to create a community website. Such communities can use a wiki to fill this need. This is especially true if a third party hosts and maintains the wiki software. One of the Mediboard community leaders mentioned that she had never created a website and that she found it "fun" and rewarding to see the content she had added looking so nice on a website. Although communities with no existing web presence may benefit substantially from wiki repositories, the experience of GSI (with a very well-maintained professional quality website) demonstrates that wiki repositories can be useful to groups with existing websites as well.

\section{Enabler of New Activities}

A few communities mentioned that they liked the idea of having a wiki because it would enable new activities that they had not been able to perform well in the past 
(although not in those terms). For example, one community that did not end up participating was excited about the idea of using a wiki to share recipes related to the disease in question. They had been using a Yahoo group database, but felt that the wiki format would probably work better. GSI had recently talked about collecting information on clinical trials, and when the wiki opportunity was explained they felt like it would allow them to do just that. Another group mentioned that they could use the wiki as an outlet for material that was removed from a book that some members had authored because of its length limitations. Although the email list and traditional publishing outlets had failed, they believed that a wiki would allow members to distribute important information. These examples show that several communities had projects that they wanted to perform, but were not yet well supported through traditional means.

\section{Other Benefits}

Communities mentioned a few other perceived benefits on more rare occasions. These included the reduction of frequent questions, the usefulness of the repository for newcomers, and the opportunity to "flush out" content from existing forums (i.e., make explicit some of the knowledge that was in the heads of members). There are likely other perceived benefits of the project that were not made explicit in the personal email messages or initial contact with members. Some of these were recognized at a later point in the project after the wiki had been implemented.

\section{Discussion of Considerations when Deciding to Adopt a Wiki Repository}

Many potential concerns were raised by communities that considered adopting the wiki. These included many of the topics that would be expected in OMSCs such as concerns over privacy, technical ability of members, and the quality of information. Interestingly, concerns about the new work required for the project were significant, even though in the css-d community the wiki was initially created to help reduce the workload 
of participants (due to its help in addressing frequently asked questions). Although one community did mention this as a potential benefit, most communities did not see this as a major selling point. This is in line with Preece's argument that FAQ documents may not serve OMSCs well (1999). Instead, the flexible nature of the wiki allowed communities to see it as helpful in supporting other worthy community goals (e.g., sharing recipes and creating useful resources for newcomers). This suggests that framing wiki repositories as an FAQ-like resource may not be as appropriate for OMSCs as framing it as another form of group collaboration than enables new activities.

One somewhat surprising finding was the extent to which communities were interested in the wiki repository as a marketing tool that could help attract members. This highlights the ongoing challenge that communities face in finding new blood to keep the communities going. It is also a problem that is particularly poignant to closed communities that have no publicly available archives or website. Although a wiki repository may help resolve the challenge of keeping members’ privacy, while still revealing enough to entice new members, most communities concerned about privacy did not see the wiki in its current form as satisfactory. Future technologies that help members strike this balance would likely be useful, as some communities are not willing to trust their members to make wise decisions about privacy on their own.

In addition, there is a need for research that helps understand how to help wiki repository readers become active email list and wiki contributors. Recent research discussed in Chapter 6 has already highlighted the fact that many people come to OMSCs primarily for information seeking purposes, but stick around for social support (Meier et al., 2007). It remains to be seen how a wiki repository can help with this transition and what content is useful for the different needs of community members. There may also be opportunities to encourage interaction between wiki readers and email list participants without requiring the readers to immediately join the email list. For example, the wiki could include a "thank you" field where visitors could write a brief thank you and have it 
sent to the email list. Or, the email list could be advertised in the footer of each wiki page with a comment indicating its potential value (e.g., "Have a question not answered here? Send it to...”).

\section{Successfully Introducing a Wiki Repository}

Deciding to adopt a wiki repository is only the beginning. The greatest challenges relate to motivating others to participate and learning the skills necessary to effectively use the wiki to the benefit of the community. In this section I describe the successes and failures of the suggestions that were provided to the OMSCs (based on the css-d study and literature review). I focus primarily on those most important in the initial stages of the wiki adoption, recognizing that future work will need to address their effect on the wiki's long-term sustainability. Thus, the goal was to answer the question: how can existing online support communities effectively introduce a wiki repository? The suggestions that were tested were provided in the wiki training pages (see Appendix 3), as well as occasionally reiterated in private conversations and email exchanges with those heading up the wiki project.

\section{Seed the Wiki with Content}

All 3 communities we worked with followed the suggestion to seed the wiki with content before "rolling it out" to the community as a whole. Those who contributed to the wiki spent considerable time in seeding the wiki with content. There was some concern with using the wiki at first, as none of the contributors had used a wiki before. I fielded several questions about how to use the wiki (e.g., how to create a new page) for early contributors of each community, although this only took at most an hour per individual (spread out over time). However, as contributors began using the wiki they typically found it easier to use than they had expected at first and concern was replaced with excitement about the project and increasing confidence in technical abilities, as well as 
more concern with the actual content being added. The following quote is indicative of most contributors’ early experience:

I think the hardest thing for me was just to feel comfortable enough to do it. It was a whole new format for me, it was a little scary and I didn't want to mess it up and once I got going and saw how easy it was it was fun...I was actually surprised at how easy doing the computer language was because I've never done it and had always been afraid to make a page or anything like that because of that... this is the first time I've ever done a board like this or anything so it was really satisfying for me.

Table 9.2 provides some quantitative measures of the seeding experience. It includes the handful of edits that I made, typically to help link pages together or fix formatting errors, except for GSI, where I was also involved with renaming some pages and editing some help pages at their request. However, I did not include myself in the “Community Contributors” column, as I was not a community member in the traditional sense. These numbers do not include all of the pre-populated content such as the help pages.

Table 9.2: Medshelf Wiki Seeding Experience

\begin{tabular}{|l|l|l|l|l|l|}
\hline Community & $\begin{array}{l}\text { Days Seeding } \\
\text { Wiki }\end{array}$ & $\begin{array}{l}\text { Community } \\
\text { Contributors }\end{array}$ & $\begin{array}{l}\text { Total New } \\
\text { Pages }\end{array}$ & $\begin{array}{l}\text { Total } \\
\text { Edits }\end{array}$ & Total KB \\
\hline GSI & 48 & 5 & 21 & 195 & 28 \\
\hline Graves & 13 & 1 & 7 & 62 & 32 \\
\hline Pain & 23 & 1 & 11 & 89 & 18 \\
\hline
\end{tabular}

As can be seen from this table, only GSI had multiple people seed the wiki with content. Having multiple individuals slowed down the process, as many decisions were discussed among different authors and some authors were slow to contribute their pages. However, the total number of new pages and edits were significantly larger than the other groups. It also included content from more perspectives (e.g., alternative and traditional treatments) and on a wider range of topics. In contrast, the moderator of one of the Mediboard groups described how the information on the wiki was "kind of one-sided at the moment” because she was the only contributor. She had drawn from her own 
experience, things she remembered wanting to see, and things she had experience with, since she was more confident writing about those.

The inclusion of more than one individual was not only important in soliciting more content, it also contributed to the likelihood of other group members participating after the seeding was done. All of the initial wiki contributors to the GSI wiki contributed content after the initial seeding period. They had already committed themselves to the project, spent time on it, and knew how to use the software. This likely helps explain why all of them have referenced the wiki in the email list conversation later and encouraged others to contribute. Because several individuals have advocated the wiki, other community members see the wiki as a group project, not just the work of a single individual. In contrast, the Graves and Pain wikis have struggled to overcome this challenge. In fact, one moderator confided that "Maybe it would have had better buy in if we discussed the outline within the group and then ask for volunteers for certain pages from the get go.” And another member who later contributed expressed frustration that she hadn't been included earlier in the process, a fact that may have contributed to her not continuing her participation for longer.

Knowing what content to initially include was a bit of a challenge for all of the communities involved. Two of the group moderators felt overwhelmed with the possibilities and asked me for help in thinking through the issue. They found my recommendation to focus on information that is commonly discussed in the community and not well covered elsewhere on the web useful. A couple of the contributors mentioned searching past archives to find content that they added, although this did not seem necessary for some who relied on their own memory of the discussion coupled with resources they already knew well. The Graves community used much of the content they had already collected in the Thyroid 101 section of the bulletin board. 
In addition to selecting content to add, members wanted to make sure it was of high quality and helpful for novices. This was a source of concern for one of the contributors, although other contributors did not voice this concern explicitly:

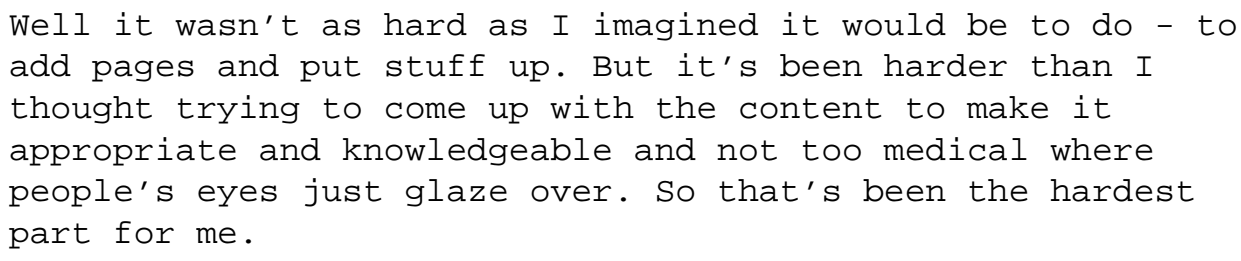

Another challenge was organizing the content. The ability to organize content was seen as a huge benefit to the wiki over the threaded conversations, and one mentioned by several members as a plus. However, it requires extra effort that requires forms of work not typically performed as part of a threaded discussion. One moderator found it helpful to use paper and pencil to help organize the basic outline, a practice which she recommended to others and seems particularly well suited to individuals without prior experience using wikis. Other group members struggled with knowing how to scope out the pages (i.e., how much was too much information for a single page). Linking pages together was also a bit confusing and an area where I felt I needed to help out. Specifically, I added inline links to other pages and "See Also" sections that link to related pages. The one area that people did well with was following the suggestion to use the Main Page as a kind of index to the other pages in the wiki. A recommendation that was not made initially, but makes sense is that (if possible) someone with prior wiki experience be involved with overseeing the organization of the wiki and interlinking of pages.

\section{Establish Wiki Related Roles}

Communities were encouraged to establish roles related to the wiki. Most importantly, we recommended that each community compile a Wiki Team (which may be only one individual if it is a small community) that would be in charge of seeding the 
wiki with content and encouraging its use. Only GSI included more than one member as part of the Wiki Team (they included 4, after one potential member declined). The GSI Wiki Team has regularly exchanged email messages to discuss the progress of the wiki project and have all fulfilled the role of encouraging the wiki's use on the email list. The Wiki Team has also partitioned the wiki into sections (e.g., the Clinical Trials and Integrative Medicine sections) that different members help keep track of. For example, one of the Wiki Team members personally contacted several clinical trial participants and asked them to share their experiences on the wiki either directly or by emailing him so he could post the information later.

We also included a Wiki_Helpers page (see Appendix 3) where members could self-identify themselves with specific wiki roles including: Clean up Crew, Promotion Crew, Page Advocate, Wiki Digest Editors, and Wiki Team members. Two members of the GSI Wiki Team listed their names as Wiki Team members, as did the Graves moderator. However, nobody in any groups listed themselves under the other groups. It is likely the case that the specialized roles were not useful at this early stage in the wiki development. It appears that having a core set of recognizable individuals as a Wiki Team that serve all of the sub-roles is sufficient at first. It remains to be seen if the specialized roles will be useful in the future as more individuals contribute and the wiki grows in size.

It is also worth noting that similar to the css-d list, there are a few individuals who actively edit the wiki, but who rarely post to the email list. Many people see contributions to the wiki as extra work that contrasts sharply with the easy, free-flowing conversation that occurs in the threaded conversation. However, there is a subset of people that enjoy the work of creating organized collections of information. Several of the most active contributors mentioned the enjoyment they receive from performing this type of work. For example, one member described how she had contracted “wiki fever” and asked how long it would last. She then mentioned that she had "found a way to actually enjoy filing 
- paper files are NO FUN after this!” She is using the wiki to help others, but also "in a selfish way [to] keep track of it so that it’s accessible to [her] and everybody else.” Another member mentioned that she had "fun" working on the project and enjoyed seeing her content "put up on a page that looks nice." Other members who actively contribute have regularly authored collections of papers in other settings (e.g., for work, school), although none of them had used a wiki before. This finding suggests that people who are major wiki contributors may have some similar skills and may find some activities more enjoyable than others.

\section{Wiki Promotional Activities}

Several activities were suggested to help promote the use of the wiki, since it is a new resource that people are not accustomed to using (see Chapter 7 for a list). One suggestion was to link to the wiki in a prominent community place. GSI did so by including it in the email list footer and the Mediboard communities included it as a link across the top of their website, as well as in "sticky" posts. Server files suggest that many people have followed the link from the Mediboard website. Another suggestion was to link to the wiki when answering questions. This has only infrequently been performed, although this may be attributable to the fact that some topics for which there is wiki content have not come up on the list during my observation period.

Although getting others to use the wiki as a resource is important, at the early stage getting them to contribute to the wiki is even more important. One of the moderators described this as her most significant barrier and explained the problem in this way:

I mean that's kind of a scary thing to try and mess with the pages and [other community members] may be like you go to the library and you get your book and you don't write in it and you don't crease the pages; and that's kind of what I think some of the mentality is - that's the library and we don't mess with it. 
Contributors were encouraged to help overcome this attitude in a number of ways. They were encouraged to emphasize that collaborative nature of the wiki, complement individuals on a message and encourage them to add it to the wiki, ask for feedback on a new wiki page (or future wiki page), and encourage contributions on a targeted "topic of the week.” The communities have done this to varying degrees with varied results. Table 9.3 summarizes some of the statistics on participation after the initial seeding experience through July $8^{\text {th }}, 2007$.

Table 9.3: Medshelf Wiki Contribution Experience after Initial Seeding

\begin{tabular}{|c|c|c|c|c|c|}
\hline Community & $\begin{array}{l}\text { Days since } \\
\text { Seeding }\end{array}$ & Contributors* & $\begin{array}{l}\text { Total New } \\
\text { Pages }\end{array}$ & Total Edits & Total KB \\
\hline GSI & 121 & 20 & 45 & 408 & 176 \\
\hline Graves & 107 & 12 & 19 & 535 & 87 \\
\hline Pain & 97 & 1 & 1 & 26 & 10 \\
\hline \multicolumn{6}{|c|}{$\begin{array}{l}\text { * Excludes myself and research assistant, as well as spammers (those whose edits were reverted). There } \\
\text { was } 1 \text { spammer for the Pain Community and } 5 \text { for the Graves Community. Number may be overstated } \\
\text { because IP addresses are shown when members make contributions and are not logged in. IP addresses } \\
\text { that only differed by the last byte were combined to help account for computers that dynamically assign } \\
\text { IP addresses. }\end{array}$} \\
\hline
\end{tabular}

The Pain Community has mentioned the wiki only 3 times in the discussion forum, all within the first month of its announcement to the list. It was not used at all to help answer questions. Instead it was announced to the list and members were explicitly encouraged to add to it. In fact, the moderator only rarely edited the wiki after its initial announcement. No other community members have contributed to the wiki in the 3 months since it was presented to the group, although a few of them have expressed gratitude for the information found on it. The Pain moderator believes that this is largely the case because the community is not very active and is hesitant to edit others' work (as described in the above quote). I would argue that the lack of new community members is also a factor, as the need to create resources useful for newcomers is not a pressing one. In addition, the wiki has not been drawn into the ongoing activities of members, most of which relate to social support rather than information sharing. This makes it difficult to follow the advice provided, since most of it relates to dealing with question and answers. 
It may be the case that a wiki repository is not useful to such a community. Alternatively, it could be that it has not yet been used for a purpose that is in line with the needs of the members and that given the right focus members would be willing to get outside of their comfort zones to contribute.

The Graves Community has been more successful in soliciting contributions, although it has been a continual struggle. The initial announcement of the wiki was relatively well received and spurred initial contributions. In total, over 10 community members have contributed over $87 \mathrm{~KB}$ of information (almost 3 times more than the original seeded content). This is likely, at least partially, due to the fact that the Graves Community is far more active than the Pain Community and includes more information sharing activities, although social support is still very prevalent. It is likely also attributable to the diligence of the moderator (and other members) in promoting the wiki. This is especially true, as the number of contributions has decreased along with the number of messages promoting the wiki. A typical message promoting the wiki is shown below:

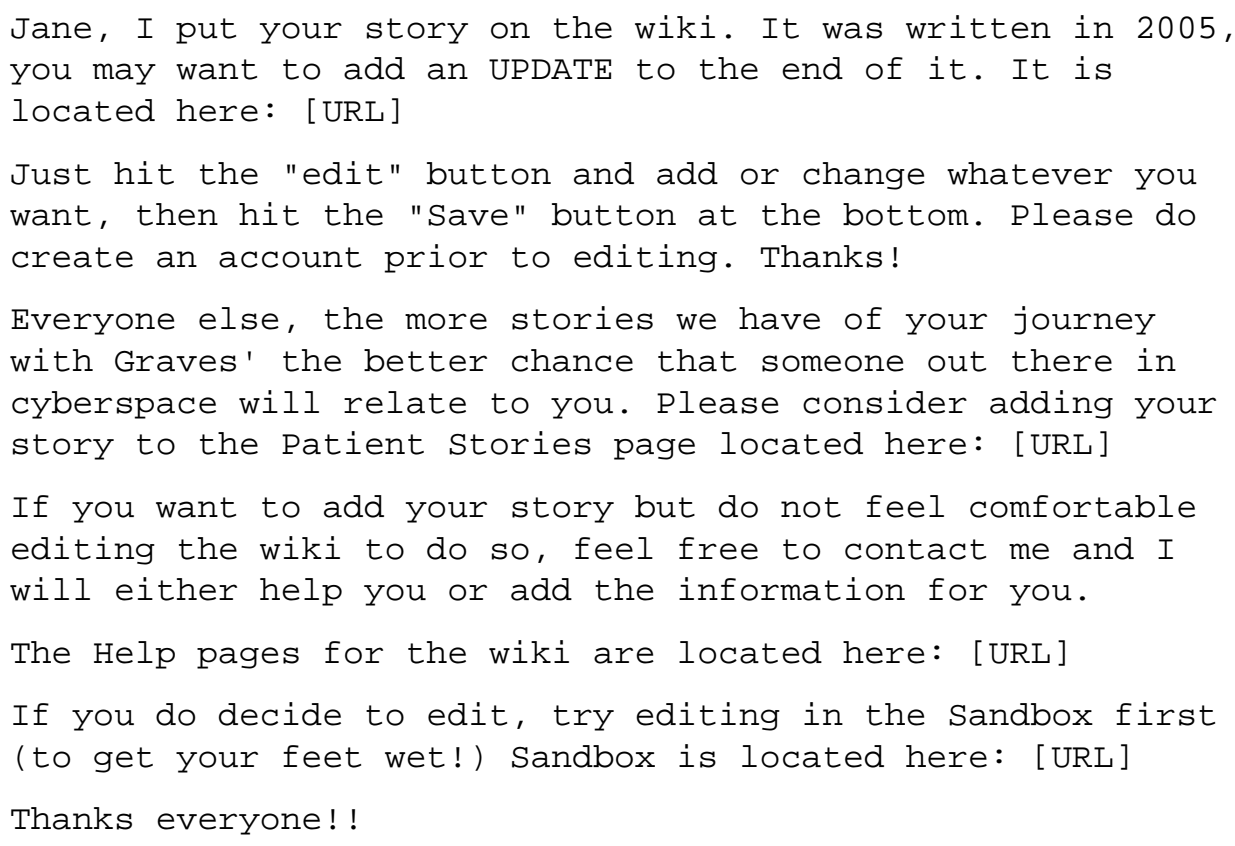


This message did prompt Jane to edit her page, as well as three others to post personal stories over the next few weeks. Although this was not as many as the moderator had hoped for, it clearly had the intended effect.

The wiki has been used in nearly every other situation that was recommended. For example, it has been used by more than one individual to help newcomers answer questions and learn more. Members (typically the moderator, but at least one time another member) have mentioned that they have added useful content to the wiki from the email list. The moderator even linked to her personal story homepage to help a newcomer learn from her experience - a mixture of both social support and information sharing. The following message excerpts are typical:

- Unfortunately my time will be limited in the short term, but that should not stop you or others from adding content.

- The wiki that I posted about last week is shaping up, but the wiki is a TEAM effort. If it is to be the wonderful resource that it can be, then we need your input as a wiki team member!

- Joseph!! Look at your join date....it's been 5 years! Time flies. Have you seen the wiki?

- Linda, do you have the link for the first article? I would like to add both of these to the wiki's Research page.

- There is some good information about Grave's nutrition under the Self Help section of this Wiki: [URL]

These examples show how members linked the wiki pages into the ongoing activities of the community. The moderator also did a good job of pointing out the importance of the project, as in the following message:

Yep, it's gonna be fun and when we are through it will be EXTENSIVE and THOROUGH and hopefully a lifeline to newly diagnosed.

When I think back to the pitiful amount of information available when I was diagnosed in $199 \ldots$ this is just a 
wonderful opportunity to make good, accurate, patient based info available to all.

There were also a number of discussions about the wiki itself. Initially members shared their excitement for the project: “THIS SHOULD BE FUN" followed by "I was hoping you would be jazzed about it as I am.” Later, one member suggested two changes to the wiki that we implemented. These included adding a footer to each page to aid with navigation and making the external links open a new window. All of this kept the wiki fresh in the member's minds. However, as the references to the wiki declined in recent weeks there has been a noticeable decline in edits as well. This suggests that ongoing encouragement and discussion of the wiki are important in keeping members actively involved with the wiki.

GSI has been the most active in promoting the wiki and has had the most contributions. Approximately 20 individuals have contributed to the wiki and a surprising 36 community members have registered on the site. There has been approximately two and a half pages created each week and over 400 total content edits. Although contributions come in bunches on given days, there has been consistent growth in the wiki since its introduction to the community and no sign of it slowing down as of early July 2007.

Like the Graves Community, GSI has followed many of the suggestions related to promoting the wiki. They have added a link to their wiki main page in the footer of their email list. They also added a link to the wiki on the main gistsupport.org website with the text “Wiki (Tips from Members).” A few members also mentioned telling other friends of theirs (typically found through other related mailing lists) about the wiki.

The wiki has also been consistently promoted to members within the community. Typically, the promotion of the wiki has been by the Wiki Team members. These members have been active in editing the wiki, as well as encouraging others to contribute through private messages and public posts. The importance of the wiki to one of these 
individuals is indicated by his practice of signing his email messages by his wiki username - a nickname that makes reference to the wiki. However, other core community members have also promoted the use of the wiki in the email list. For example, one of the list founders has sent 2 messages encouraging members to contribute to the wiki and explaining what it is, despite the fact that he has not contributed himself (a fact that others have no way of knowing since many contributors don’t login). The buy-in from the most active and respected members in the community has clearly helped motivate many individuals to at least try the wiki, as evidenced by the fact that 36 members have registered on the site (although only around 20 have contributed).

As with the Graves Community, the GSI wiki is mentioned regularly in the email list as members use it to help answer questions, encourage others to contribute to it, announce new pages (and ask for feedback on them), and discuss its potential. Requests for new contributions on a specific topic have been successful at encouraging others to participate. The following example demonstrates how this has been implemented by GSI members:

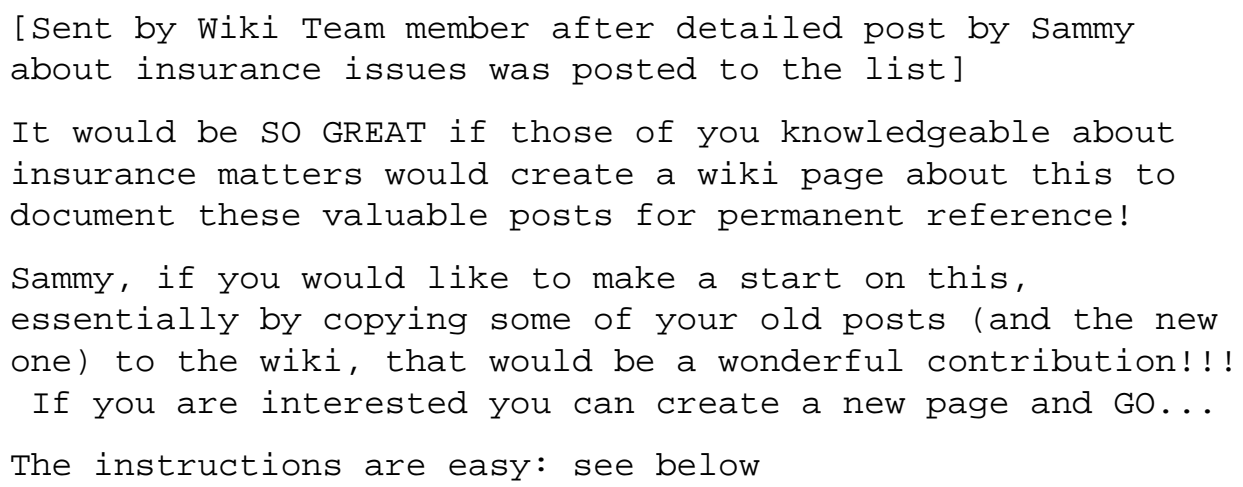

This email was acted upon by Sammy, who added content to the wiki pages that the Wiki Team member had created for her. A few days later the Wiki Team member posted another message announcing that Sammy had created some insurance pages, thanking her (“Go Donna!”), and encouraging others to add to the wiki by copying their 
posts or embellishing them. This solicited another reply by a member who had made his first wiki edits (as a result of a prior call for participation in the wiki by the Wiki Team) a few days prior and wanted to encourage others to edit the wiki as well:

Yeah Sammy!

I just want to encourage everyone to get in and add to those WIKI pages. There is so much info we have in this list and so little of it has made it to those pages. I went in and made some serious additions to several sections Fatigue, Iron, Omega 3, etc. If everyone does that it could be a great resource.

Although these messages have encouraged participation of new members, the process is slow and the vast majority of content is still entered by Wiki Team members.

Although most edits are made by Wiki Team members, they often contain information from others’ email list messages. For example, one Wiki Team member has taken ownership of a subset of pages. He has convinced a few members with relevant experience to post to the list, however, the majority of contributions to the pages are from members that email him their comments and/or give him permission to add their list messages to the pages. This strategy seems to have worked well, although it requires more of this Wiki Team member's time.

In summary, it appears that members need continual encouragement to post to the wiki, as well as reminders that it exists. The communities that have solicited the most community involvement in the wiki (i.e., GSI and Graves Communities) have encouraged their members the most and tried to link the wiki content to the ongoing discussion. This helps confirm that the specific practices suggested to the communities (e.g., announce new wiki pages, recommend particularly useful messages be added to the wiki, link to the wiki in message footers, emphasize it as a community project) can be naturally adopted in communities other than css-d. In addition, there is some hint that the wiki information can be useful in providing emotional support, as in the Graves Community example 
where a member linked to a personal story wiki page to help provide both information and emotional support.

\section{Wiki Digest}

One suggestion made to the communities was to have a "Wiki Digest" where new content was highlighted, frequent editors were recognized, and pages that need work were identified. None of the communities sent out a Wiki Digest. This was partly because it was time consuming and partly because there didn't seem to be enough wiki activity to warrant it. However, GSI did think it was a good idea to send out a specific topic and encourage people to contribute to pages on the topic. They did this once on June $10^{\text {th }}$, resulting in some activity by the regular contributors, as well as 2 new wiki contributors. Overall, the approach seems to have helped some, but not been as successful as the core members would have liked. It remains to be seen whether or not this approach is useful for more established wikis.

\section{Relationship of Threaded Conversation and Wiki}

The Wiki Guide page (found in Appendix 3), where the wiki is explained to newcomers, describes how the threaded discussion and wiki differ from one another. It describes the email list as the place where personalized questions are asked and answered, second opinions are sought, social support is given, and new information is announced. In contrast, the wiki is described as a place where content of lasting value that is general enough to serve many people is stored. It is a collection of information resources. Ideas are provided on what benefits each one can derive from the other. In addition, members are encouraged to summarize email list content when adding it to the wiki, as well as remove personal information. Likewise, they are encouraged to discuss wiki content in the email list. 
All of the communities have accepted and stuck to this general description of the wiki and how it relates to the email list, although they have not always followed the advice to remove personal information and distill the original messages. Those involved with seeding the wiki and introducing it to the community have described it as a "community library" and a collection of "tips from members." Many of the pages that were seeded on the wiki initially were summarized and private information was removed. However, later additions often are just a copy and paste of the original message, perhaps with the name of the author removed, although not always. For example, one of the GSI insurance pages begins: "After dealing with insurance companies as my job for many years I have a few suggestions when an insurer turns you down for a procedure or treatment or medicine...” It continues in the first person, but does not include the information about the author, which was included in the email message it was copied from but is missing in the wiki version. Other pages discussing clinical trials also include exact copies of messages with no editing, sometimes with the author's name included.

Part of the reason content is not edited before adding it to the wiki is that those adding the content are copying it from other people's posts. They have retained the exact wording in order to assure that they are not misrepresenting the information. It also assures that they are not introducing inaccurate information - especially if it is on a topic that they don't know a great deal about. This also helps explain members' hesitancy to edit content on some pages, a hesitancy that was expressed in multiple interviews and seems to be a bigger issue than in the css-d community where members rarely expressed concerns about editing others' work. In addition, members copy and paste because it is (as one member described it) the "easy way out."

It may be more difficult to summarize some types of information shared in OMSCs because of the way it is typically shared. As discussed in Chapter 8, it is common for members to share personal experiences in OMSCs. This makes it challenging to know if the individual's experience generalizes or not. In the discussion, 
members share different stories and the burden of deciding whether or not the information is applicable falls on the reader. What's more, readers are typically only interested in the information applying to their own situation, not if it "generalizes" to many individuals. There seems to be a fear that if content is distilled and removed from its original context too much, members may apply it to situations where it was not intended. While this is also the case in other communities (e.g., css-d where details about a technique's usefulness in different browsers is frequently stated), the hesitancy of nonprofessionals to prescribe solutions to medical problems is greater due to the potential for ill effects from incorrect (or inappropriately applied) information. This suggests that OMSCs may benefit from carefully worded summaries (e.g., "some members find that..., although this may only apply to...”) attached to actual messages related to the topic.

\section{Consider \& Support Wiki Genres}

Another group of suggestions provided to the communities related to the use of Wiki Genres. A collection of different wiki genres was provided, with examples of different genres found throughout the web (see Appendix 3). For example, wiki genres included the How To Genre, Links Genre (similar to the “Annotated Links” in css-d), Encyclopedia Genre, and Essay Genre among others. Wiki contributors were encouraged to consider which wiki genre would best fit the information they were going to contribute, as well as help spur new ideas for content. Although some pages followed patterns similar to those shown in the recommended genres (e.g., the "links genre” and the "essay genre" - in the form of personal stories), there is no evidence from any discussions or interviews that the idea of genres was particularly helpful or useful for contributors. Two community leaders had not read the Wiki Genres page (or had forgotten reading it) even though it was recommended on the Getting Started page which they had used. This may be because of the information overload problem discussed earlier. 
Instead of well thought out wiki page genres, most pages are best described as a smattering of information on a particular topic. The information is often copied in from discussion threads (as described previously) and at most organized into sections. A single page often includes both first person and third person paragraphs, with no transitions between them. In addition, no structured formatting (e.g., a table) has been used to help present data except for bulleted lists. All of this suggests that members pay little attention to the presentation of data, when compared with the actual content. The impact of this on readers remains to be seen.

\section{The Need for Patience}

One recommendation that was not discussed initially, but was later made based on feedback from the communities was to stress the importance of patience in the process of introducing the wiki. All of the community leaders involved with introducing the wiki have been disappointed at times with the lack of activity on the wiki. Although this concern is well founded for the Pain Community, the activity level of the Graves and GSI communities is comparable with the early experience of the css-d community. Indeed, the css-d experience showed that a highly useful wiki could be cultivated by a community through relatively infrequent (but regular) and small additions over an extended period of time. I found myself encouraging these leaders by sharing this story, in the hopes that they would not want to prematurely end the project or feel like they had wasted their effort.

Table 9.4 demonstrates that two of the communities have added content at an average rate that is comparable to the css-d community’s early experience. Data includes the initial seeding experience, as well as the first months of use for all communities, although the time duration differs for each. Unfortunately, the css-d data did not allow us to separate out the seeding and non-seeding time periods. 
Table 9.4: New Wiki Content Growth (Including Seeding Experience)

\begin{tabular}{|l|l|l|l|}
\hline Community & $\begin{array}{l}\text { Number of Days } \\
\text { in Corpus }\end{array}$ & $\begin{array}{l}\text { Average New } \\
\text { Bytes per Day }\end{array}$ & $\begin{array}{l}\text { Average New } \\
\text { Pages per Week }\end{array}$ \\
\hline css-d & 256 & 1,067 & 5.9 \\
\hline GSI & 169 & 1,235 & 2.7 \\
\hline Graves & 120 & 1,018 & 1.5 \\
\hline Pain & 120 & 247 & 0.7 \\
\hline
\end{tabular}

After this initial period of relatively rapid growth, the css-d community settled down to a much lower average growth rate of 437 bytes per day and 2 new pages per week. This suggests that communities should focus on maintaining participation over the long-run, instead of expecting significant daily contributions. In other words, there may be a tendency to want to quit the projects because of an ill-founded suspicion that the project will fail due to the slow growth rate.

Another approach I used to help early contributors overcome their concern that the wiki was not moving ahead fast enough was to point out its value to non-members. I did this by reviewing server logs and pointing out to them that people were finding their wiki through search engines. I also highlighted pages that were of interest to those who found the site. The community leaders were very interested in this content and two of them forwarded my message on to their community to help provide some validation of the work that was being done and encourage more participation. Although rare, a similar occurrence at css-d happened on at least one occasion. One of the administrators mentioned that the IE7 page that the community had created was being used by the Microsoft IE7 developers to help improve its support of CSS. These examples show that demonstrating the value of the repository to outsiders is an important motivator of contributions and validation of prior work.

\section{Discussion of Introduction of Wiki Repository}

Many of the original suggestions derived from the css-d group were adopted by at least one of the 3 participating communities. This suggests that they are possible to 
implement in more than one type of online support community, and indeed seemed reasonable to leaders of those communities. They also had the desired effect in most cases. The key suggestions included seeding the wiki with content, promoting the wiki using various methods, clearly identifying activities appropriate for the wiki versus the email list, and establishing a Wiki Team to help promote the wiki. Other suggestions like posting a Wiki Digest to the email list, allowing for members to sign up as Wiki Helpers, and actively considering Wiki Genres were not successful. Interestingly, these suggestions were innovations that I had developed, not best practices already in use by the css-d community. This suggests that the method of identifying successful practices from an existing community is a good one that is likely to lead to implementable suggestions.

The experience in rolling out the wiki to the participating communities also helped inform and refine some of the suggestions. The importance of including multiple individuals when seeding the wiki became apparent. It helped improve the diversity of the information and make it seem more like a group project than an individual one. It also helped after the wiki was introduced to the community because there were several members who knew how to contribute, had a vested interest in the project's success, and could help encourage the wiki's use. The failure of the Wiki Digest suggested that it was not appropriate at an early stage, although one of the ideas from it (the topic of the week) was at least tried by one community with moderate success. This may also be the case with the Wiki Genre information. Future research will need to determine if those ideas are useful later or more effort than they are worth.

One particularly challenging issue that arises because of the medical context has been the hesitancy of members to edit others work. This manifests itself in the direct copying of material from email messages into the wiki, as well as members' general hesitancy to edit other members' wiki contributions. At least part of this hesitancy seems to come from the concern that decontextualizing the information will remove something 
of value, such as the information that allows others to know if the content should apply to them. It would be valuable to understand what types of information are best kept as contextualized wholes (e.g., illness narratives) and which types of information can safely be decontextualized (e.g., collections of links to other resources). It would also be worth exploring technologies that help support the need of individuals to determine if a specific suggestion is applicable to their own situation. For example, a system may automatically determine that you share similar, relevant characteristics with an individual who has responded well to a particular drug regimen. Or, conversely, it may alert you to the fact that the person recommending a particular activity has a different underlying condition that makes his recommendation meaningless for you.

Overall, the experience of implementing a wiki repository with 3 new groups confirmed that the greatest challenges in creating a useful community repository relate to motivating others to contribute. With proper motivation, even non-technically trained individuals were completely capable and willing to learn how to use a fairly complex wiki system. However, convincing a significant number of members to contribute is an ongoing challenge that requires constant ongoing effort. It is also one that can feel like it is failing in the moment, even when it is successful in the long-run. This suggests the need for research that helps uncover technical and social recommendations that reduce the cost of wiki contribution and increase the benefits, or at least make them more apparent. For example, the presentation of server log data to communities seems to have helped validate past work and motivate further contributions. The Wiki Digest was intended to serve a similar need (by recognizing member's contributions), but the effort required was too much work too early. Perhaps automated systems that highlight wiki contributions and send them to the list would be helpful (e.g., "A new wiki page DrugInteractions has been created by JoeSmith”).

It is worth highlighting that many of the greatest benefits of the css-d wiki were a result of its reuse in the ongoing conversation (see Chapter 6 for details). For example, 
many core members were motivated to contribute to the wiki so they could later refer to it and wiki content was better maintained when it was reused. The prior section showed that several communities were more interested in wiki repositories as a way of enabling new activities than they were for reusing the content in the ongoing convnersation (e.g., to help answer frequently asked questions). If this is true more universally, it suggests that different strategies may be needed to help overcome the challenges of motivating contributions and maintaining content. At this point, it is too early to definitively say what the different strategies might be. However, the willingness of community leaders to spend so much effort in seeding the wiki content suggests that at least some individuals are highly motivated in the medical arena. The key is to get the entire community involved. Future research will examine the success of communities over time in order to see what strategies were helpful in getting more community involvement when the wiki is not primarily concerned with reuse in the conversation.

\section{Lessons Learned}

In addition to the findings related to the research questions, I learned a great deal from the process of performing an action research project in an online community environment. Below is a summary of the major lessons I learned from this experience.

\section{Recruitment}

Some communities wanted to see a working example of a repository that was already in use. Because the technical and medical contexts were so different, it was a challenge to portray exactly what the repository could look like in a medical context. To help overcome this challenge I created a sample "Main Page” of a wiki repository that listed several hypothetical pages of a medical nature (see Appendix 3). However, this was not completely satisfactory, as each individual community has its own unique needs. Initially, I discussed possible content ideas with group leaders, which seemed to work 
well. Once one community had adopted and seeded its wiki it was then possible to let others look to it as an example and a starting point for a discussion of how it might help their own community.

I found it helpful to speak with potential community leaders by phone when possible. However, several communities had a group of leaders that all wanted to be in on the conversation. In those situations we used email to communicate with all of the leaders. The challenge with that approach is that possible concerns can't be resolved right away and it is less personal. In one case we used a phone conference with 3 of us, which worked well.

The recruitment experience demonstrates that communities recommended by others are most likely to consider participation in the project. Importantly, two of the communities we ended up working with were based on referrals from a community leader of a group that chose not to participate. Since many members participate in multiple online communities, this suggests that recruitment materials should ask list owners to share the message with others that they believe would be interested.

Members cared greatly about the long-term sustainability of the project. To help convince them that they would not be wasting their time creating a bunch of content that would be removed later I did several things including: committing to fund the first year, using open source software (that they could install themselves for free if they wanted to later), making our modifications to the software easily implementable (as a "skin” to Mediawiki) and publicly available, and promising to export the database so that they could import it into their own install of the software and not lose any content. This approach seemed to work well in overcoming most of the concerns.

One reason that we were able to successfully recruit communities is that they had little to lose from attempting the project. This was due to the fact that we did not require them to change the current email list or bulletin board software that they used. This approach did not allow testing of some novel designs that would require a new system be 
adopted, but it made it possible to convince communities to participate and was ethically responsible in that it did not introduce a significant disruption to the existing community for an unproven innovation.

\section{Implementation}

It became apparent after working with the groups that I had provided too much information to them initially to help them get started. Some of the content that was not immediately applicable was not used by them because of this. I fielded several questions that were addressed in the written materials (i.e., wiki help pages and best practice pages). In hindsight, I would have provided some suggestions at a later time period, once they were more applicable (e.g., the Wiki Digest).

A fair amount of effort was required to create and customize help pages and improve the general usability of the website. This work was necessary to make the wiki repositories possible to use in practice, but did not contribute to research in and of itself. Even with the effort that we put in, we recognized other possible improvements that were not feasible to implement given our time constraints. This suggests that when performing this type of project it is important not to underestimate the importance (and required effort and funding) of creating a usable system. 


\section{CHAPTER 10 \\ CONCLUSIONS}

Several common themes have repeatedly shown up throughout this dissertation. In this chapter I highlight some of those key themes and specifically focus on their implications for design. I also suggest some valuable lines of future research.

\section{Online Support Communities' Place in the Broader Information Landscape}

Members of online support communities see them as a unique source of practical knowledge on specific subjects, whether it is website design or cancer treatment. As we saw in Chapter 4, the threaded discussion format is well suited for highly personalized questions and responses, announcements, and meeting other immediate needs (e.g., the need for social support or feedback on a website layout). The result is that the community discussions help draw out the expertise of knowledgeable members in a public setting, while addressing the topics and needs of novices. Thus, they are highly responsive to new issues that arise and often cover topics in great depth and at a level that is useful to novices. Also, because they are a natural place to come for answers to questions, their discussions often reflects topics that are not addressed elsewhere, or are too difficult to find elsewhere. As a result, their content naturally fills existing information gaps.

Unfortunately, without a way of summarizing and de-contextualizing the information from the conversation, information of potential use often remains hidden or unusable. The need to address this concern is what originally led to the creation of traditional FAQ documents, an early form of a community repository that has proved its worth over time. This dissertation has demonstrated how a wiki repository can meet a 
similar purpose in a much richer way. Thus, the combination of a support-based conversation and a wiki repository can lead to the production of succinct, relevant content that is highly responsive to the needs of the many (e.g., novices) and help fill important information gaps. Furthermore, the processes described in Chapter 6 help assure the quality of the wiki repository information, as it is constantly refined because of the feedback members receive about it in their ongoing conversation.

Although several wiki repository genres were identified in the css-d study (see Chapter 5), many of them were similar in that they helped aggregate knowledge that was highly distributed among individuals and existing resources. For example, some of the most useful pages are annotated links to other resources (e.g., 3-column webpage design templates) or collections of different techniques for achieving a certain effect (e.g., creating rounded corners). In fact, several members mentioned that this type of activity was valuable and one of the key things that differentiated the wiki from other CSS-related resources created by individuals and communities. This ability to aggregate disparate knowledge and succinctly present it was facilitated by the fact that there was an entire community contributing and refining the pages. The ability to structure content (by using tables, headers, and bulleted lists) was also important in presenting this aggregated information in a coherent manner that facilitated comparison (e.g., see ThreeColumnLayouts page in Chapter 5). This suggests that community-driven content may be particularly good at collecting and presenting diverse knowledge that is widely distributed among individuals.

In short, there is great value created when groups of individuals share information (e.g., ask and answer questions) in a public forum - especially when tools and social practices help refactor it into a more reusable and distilled form. This approach can be used in many settings where it is not currently used. For example, thousands of information professionals (e.g., librarians, archivists, technical support personnel) answer questions in private settings, making it impossible for others to learn by "overhearing” 
their conversations. Providing tools that bring some of these discussions into the open, as well as helping distill them into useful, shared resources would help tap into great reservoirs of knowledge - reservoirs that stay full no matter how many drink from their waters. However, the MedShelf study demonstrates that not all conversations are desirable to bring out into the open (e.g., those dealing with personal medical information), even though many participants want to let others overhear them in order to learn from them or to encourage others to join the conversation. This suggests that tools and social practices that help protect the privacy of those conversing, while still allowing others to learn from their conversations (perhaps after they have been distilled and depersonalized) would be welcome. Additionally, tools and social practices that help capture audio conversations occurring in real time and convert them into a more reusable format would extend the reach of support communities.

\section{Synergies of Community Conversation and Wiki Repositories}

The css-d study emphasized the synergies that exist between the community’s conversation and its wiki repository, and the ways that activity was organized to leverage both resources. Although the two resources were technically separate (e.g., required different logins), they were socially connected through an overlap in contributors (i.e., Bridge Builders) and mutual referencing. The discussion in Chapter 6 describes in detail the many ways that the community converstion and the wiki repository impacted one another and argues that their relationship is a mutually beneficial one. Rather than restate those arguments here, I contrast the mechanisms that led to the success of online support community repositories with those of Wikipedia, although my characterization of Wikipedia is tentative at best. I then propose a theoretical framework that could be useful in future studies of online communities that include both conversation and more lasting community information resources. 


\section{Wiki Repository and Wikipedia Comparison}

The factors that have led to the success of the css-d and Meshelf wikis are significantly different than those that lead to success at other wikis such as Wikipedia. Although they use a similar technology and also rely upon a community of contributors, their purposes and the mechanisms leading to their success are completely different. This is supported by the fact that almost nobody at css-d (and the Medshelf communities) monitors the wiki's recent changes, a practice that is the lifeblood of editing at Wikipedia. Likewise, the css-d wiki is sustained with relatively few editors (and edits), whereas many believe that Wikipedia is sustained by its overwhelming popularity.

Throughout this dissertation I have argued that wiki repositories are most successful when closely associated with a community conversation. The css-d wiki's uniqueness and usefulness came from the fact that it was a reusable object that was editable. The css-d wiki content was created and maintained because it was useful as a reusable resource in the ongoing email list discussion. New pages were created so they could be referred to later to address frequent questions. Contributors were motivated by the potential for reuse, and the content that was created was designed for later reuse (e.g., decontextualized, summarized, aggregated) and encapsulated in a wiki genre appropriate for its particular purpose. The Medshelf communities were less concerned with reuse. Instead, they saw a wiki repository as an enabler of new community activities and a source of new members. Despite their differences, each community has used the wiki to serve the needs of the community that are not well met through the conversation, but are related to it.

The key technical difference between a wiki repository and Wikipedia is that in Wikipedia conversations are attached to individual wiki pages (via "talk pages"). Another key difference is that activity is organized primarily around collaborative authoring at Wikipedia rather than question asking and answering in online support communities. 
These differences have several implications. Unlike wiki repositories where the topic of a new page is typically determined by information seekers (although experts typically create the content), at Wikipedia new content is suggested by information providers who want to share their knowledge on a topic they find interesting. Thus, when a student doesn't find an answer to his question on a Wikipedia entry he is likely to look somewhere else. When a student doesn't find an answer to his question at css-d, he is likely to ask the list for help in answering it - a process that provides a signal to the list that the topic is of interest and may warrant a wiki page. At Wikipedia content is not maintained because of its reuse, as it is at css-d. In an online support community an individual can express his concern about a wiki page's content to the list and a completely different person can fix the problem. In contrast, at Wikipedia the same person who recognizes a problem must also know the answer to fix the page. Another difference is that the content at Wikipedia is determined by how well it matches a predefined genre, not how well it fits the needs of its users. This makes it more likely that pages at Wikipedia will have a consistent look and feel, while limiting the number of purposes that content can be used for. In short, although wiki repositories are not as professional looking and consistent as Wikipedia, they are far more responsive to the needs of their users and flexible in the ways in which they meets those needs. This is the great promise of community wiki repositories.

\section{Community Conversation and Repositories as a Symbiotic Relationship}

In this dissertation I have described the synergies between wiki repositories and support-based conversation, only one of the many possible contexts and combinations of conversation and documents of more lasting value. The success of the arrangement suggests that the interplay between conversation and documents be studied in other

online community contexts. The somewhat stylized comparison of wiki repositories and Wikipedia above showed how this might be done. However, there are few theoretical 
frameworks that address the relationship between conversation and information resources of a more lasting nature. In this section I discuss a few relevant theories and then propose an alternative. Specifically, I propose that communities with a conversation and set of information resources be viewed as a symbiotic organism living in the broader information ecology.

\section{Participation and Reification}

In his book Communities of Practice, Wenger goes to great length to describe how meaning is "negotiated” through the interplay of participation and reification (1998). Why is meaning negotiated? Negotiation connotes “continuous interaction, gradual achievement, and give-and-take” (p 53). Meaning must be negotiated because "meaning is not pre-existing, but neither is it simply made up. Negotiated meaning is at once historical and dynamic, contextual and unique” (pp 53-54). In fact, meaning exists in the very process of negotiation. To understand this negotiation it is necessary to understand the interplay between participation and reification.

Participation suggests "action,” or the process of taking part in some activity and "connection,” the relations with others that reflect this process. It is both personal and social and "is a complex process that combines doing, talking, thinking, feeling, and belonging” (p 56). Reification is "the process of giving form to our experience by producing objects that congeal this experience into 'thingness'” (p 58). In other words, reification describes how "we project our meanings into the world and then we perceive them as existing in the world, as having a reality of their own” (p 58). This provides a "shortcut to communication" and includes things like "making, designing, representing, naming, encoding, and describing, as well as perceiving, interpreting, using, reusing, decoding, and recasting” (pp 58-59).

Wenger sees participation and reification as "both distinct and complementary" and part of a pair (p 62). They are a duality. One cannot be understood without reference 
to the other. "They come about through each other, but they cannot replace each other. It is through their various combinations that they give rise to a variety of experiences of meaning” (p 62). Wenger also sees them as being complementary in that they "make up for their respective limitations” (p 63). Participation repairs the potential misalignments inherent in reification by coming to the rescue when "the stiffness of its form renders reification obsolete, when its mute ambiguity is misleading, or when its purpose is lost in the distance" (p 64). Likewise, reification comes to the rescue when "the informality of participation is confusingly loose, when the fluidity of its implicitness impedes coordination, when its locality is too confining or its partiality too narrow” (p 64).

The interplay between reification and participation is closely related to what Giddens calls structure and agency, which he also sees as a duality (the duality of structure) (1986). His theory of Structuration emphasizes how structure is both an input and an output of human actions. Several authors have applied Structuration theory to technology use within organizations, although technology was not a part of the original theoretical development (DeSanctis \& Poole, 1994; Orlikowski, 1992, 2000). It has also been applied to the way in which individuals enact shared genres in their communication (Yates \& Orlikowski, 1992). Using Wenger’s terms, communicative genres (e.g., an internal memo) serve as reifications of a particular way of communicating. However, like Gidden’s structures, they are constantly reinforced or modified as they are enacted (through participation) in various situations.

There is a practical payoff of viewing the negotiation of meaning in terms of a duality of participation and negotiation (or agency and structure). It allows us to consider the unique contributions of participation and reification in the production of meaning of a particular object (e.g., a wiki repository entry) or action (e.g., editing a repository page). It allows us to ask if "too much reliance is placed on one at the expense of the other" (Wenger, 1998, p 65). And, it suggests ways that certain designs may enable or frustrate the negotiation of meaning and thus, learning. 
So how do community conversation and repositories relate to this framework? It is tempting to associate conversation with participation and a wiki repository with reification, but this would be incorrect. As was discussed, each object and action includes aspects of both, and they describe an interplay, not a classification scheme (p 68). However, it is appropriate to ask if various designs place too much reliance on participation or reification and try to unpack the implications of this on the community. Likewise, it is appropriate to ask what the implications of a certain design suggestion would be on the negotiation of meaning through participation and reification.

Using css-d as an example, participation was manifest in the conversation in many ways: asking and answering questions, diagnosing errors, providing references, disagreeing with others' suggestions, finding CSS errors on people's websites, as well as developing an identity as the "problem solver” or a lurker. Reification was manifest in the conversation through the use of shared techniques for solving problems, specialized vocabulary terms like "holy war" and their application to designated topics such as determining the optimal font size, accepted communication genres such as "site checks," and shared stories about the evils of HTML table-based designs.

The css-d wiki enabled new forms of both reification and participation. Although the primary goal of the wiki was to capture some of the conversation in a way that would lend itself to later reuse (i.e., to reify some of the ephemeral content), it quickly became apparent that participation was dramatically altered. The wiki enabled (and encouraged) a whole host of new forms of participation (i.e., activities) such as summarizing and distilling prior conversations, collecting materials, collaborating to create a shared document, organizing content, editing content to make it more readable, deleting spam, linking to repository entries in the conversation, and recommending material for the repository. It also changed some existing forms of participation (e.g., answering a question now might require visiting the wiki beforehand to collect the appropriate URL). Likewise, new manifestations of reification were enabled including the concept of the 
community wiki itself and new genres such as Annotated Links, Debate pages, and Biography pages. Similarly, some old reified concepts were transformed (e.g., an “appropriate question” now included the need to consult the wiki before posting), while others remained unchanged (e.g., site checks).

This analysis shows the complex, ongoing relationship between reification and participation and that the introduction of a wiki repository to an ongoing conversation affected both significantly. I initially expected the introduction of the wiki repository to shift the balance away from an over-reliance on participation at the expense of reification. However, in hindsight I realize that both participation and reification were enabled by the new technical infrastructure and that the most significant changes related to participation, not reification. This is in stark contrast to a traditional FAQ document that is typically authored by one individual, which significantly limits the new forms of participation that it enables.

Symbiotic Relationship between Conversation and Repository

Although Wenger's participation and reification framework can help understand the changes that occur with the introduction of a community repository to a conversation, it does not directly address the relationship between the conversation and the wiki repository. Participation and reification are general enough concepts that they apply to any community of practice setting. However, they may not be sufficiently tailored to capture the specific relationship between threaded conversations and wiki repositories. It is thus, worth considering other concepts and analogies that may provide a more direct correspondence to the relationship between a community conversation and a wiki repository.

One such analogy comes from ecology. Let us consider the community discussion and repository as a symbiotic relationship. By this I mean the discussion and wiki repository are qualitatively different, yet interdependent. A symbiotic relationship can 
take many forms including mutualism (where both organisms benefit from the relationship), parasitism (where one organism benefits while the other is harmed), and commensalism (where one organism benefits while the other is indifferent), among others. Although the relationship between a community’s conversation and repository are ideally mutualistic, examples of the other forms of symbiosis exist. For example, individuals may not join a community conversation because their immediate information needs are met through the wiki repository, leading to a decrease in new membership that may eventually lead to the downfall of the conversation.

As with many symbiotic organisms, the discussion and repository may co-evolve as each one adapts to changes in the other. For example, content in the repository is largely driven by the topics that are discussed in the conversation (e.g., frequently asked questions). Similarly, the conversation will focus on meeting the needs that are not well addressed by the repository such as diagnosing problems and tailoring information to the needs of an individual, instead of providing background material that is essentially the same for everyone. Although the symbiotic relationship may be tenuous at first, as time progresses individuals learn to more effectively use the repository and conversation, each with their relative advantages. This has the result of strengthening the co-dependence of the relationship.

Just as symbiotic organisms exist in a larger ecosystem, a "symbiotic community" also exists in a larger social and informational ecosystem. Environmental factors may impact the conversation or repository, or even the community as a whole. For example, early members of the css-d community attribute the immediate success of the email list to pent-up demand. In ecological terms, the css-d community was successful because it filled a unique niche in the broader ecology (i.e., information landscape). Another “environmental” factor of significance was the widespread use of wikis. As sites like Wikipedia have become increasingly popular, more and more individuals have become comfortable using wikis. This broader change in the information ecology has resulted in 
more participants contributing to the css-d wiki and the importation of social norms and procedures from other popular wiki sites like WikiWikiWeb and Wikipedia (e.g., the use of categories and NPOV - neutral point of view).

The ecological analogy of a symbiotic "community" found in a larger social and informational ecosystem raises certain questions that may be particularly useful to designers. How is the conversation benefited (or harmed) from its close association with the repository? How is the repository benefited (or harmed) from its close association with the conversation? How are the two linked together? For what does one rely upon the other? Have they become more co-dependent over time? How are they related to other entities in the larger ecosystem? What environmental factors affect the conversation, the repository, or both? How will these factors affect the conversation and repository’s mutual relationship? How will they effect the community’s sustainability?

Answering these questions for a variety of different communities with different combinations of conversation and lasting documents could help define the design space surrounding conversation and more permanent documents. Like a field study of related species, this dissertation addressed many of the questions outline above in two different domains: technical and medical support. It was apparent that the different knowledge domains impacted the relationship between the conversations and wiki repositories. For example, my somewhat preliminary data suggests that decontextualizing medical content may be more challenging than decontextualizing technical content, since medical content is often shared via stories - an indication that the context around a particular insight is so important. In addition, medical communities include significant amounts of social support, which may or may not be convertible to a wiki repository. Future work in this area would help characterize these and other differences, as well as the core similarities.

\section{Designing for Reuse}


As discussed throughout this dissertation, conversations occurring in online support communities are highly contextualized and typically relate to addressing the immediate information (or social) needs of an individual. Threaded conversation supports these needs well. As discussed earlier in this chapter, these conversations also make explicit important information that is often not available elsewhere. Thus, the original conversations are an important resource called upon by other information seekers and gatherers (i.e., lurkers and individuals searching through the discussion archive). Indeed, the most common use of most discussion threads at css-d was later access of them in the archive by those not participating in the original thread. However, as we saw in Chapter 4, many of the very things that make the discussion successful at meeting the needs of the original information seeker make it poorly suited for its secondary use. This underscores an important design challenge: How can we support ongoing conversation with its social nuances, highly contextualized discussions, and immediacy, while at the same time supporting reuse of that conversation?

There are several strategies that have been used to improve the reuse of conversation. One approach has been to help the "re-users" find relevant messages. This has been done through a variety of information retrieval techniques (e.g., natural language processing) or with the help of user-provided ratings and recommendations (e.g., Resnick, Iacovou, Suchak, Bergstrom, \& Riedl, 1994). The problem with all of these approaches is that it assumes that the answer can be found in the conversation in its current form. This study has highlighted the fact that even perfect recall and precision will not suffice in many situations, because the answer is too contextualized - especially for a novice who may not know how to draw out the key concept and apply it to his own situation.

Another approach has been to automatically provide context around conversation through various visualization techniques (e.g., Smith \& Fiore, 2001). This can be useful in helping newcomers locate relevant messages and understand some of the social context 
around a message (e.g., it is sent from a frequent poster and is the $4^{\text {th }}$ of 16 messages in the thread). This can be important in determining the credibility of information and perhaps even its applicability to one’s own situation. However, as before, it does not directly address the need to decontextualize and recontextualize the content to match the reuser's needs.

A third approach is to change from a focus on reusing content to reusing individuals. For example, members with questions can be directed to automatically identified experts (Zhang et al., 2007). This approach helps questioners receive highly contextualized answers, but it requires significant effort by experts. It also suffers from the fact that many members will not be willing to wait around for an answer or will not want to "bother" an expert.

A final approach that has been attempted is to provide tools to an individual (or small group of individuals) to help them transform a discussion into a more summarized (and thus reusable) form. For example, prototype systems provide tools for organizing threads by assigning them hierarchically organized keywords (Brewer 2000) or rearranging threads and adding summary nodes with notes (Ackerman et al., 2003). These and related systems are promising for many situations where a single individual (or small group) is highly motivated to perform this work (e.g., they are paid to do so). Because they have not been created with large-scale collaboration in mind, they may not scale. For example, they do not have the ability to track changes of other individuals, a practice that is important when there are more than a few contributors. However, the techniques they use could be helpful in initially generating wiki content or transformed into a more scalable system.

The approach discussed in this dissertation, and shown to be successful in certain situations, is to encourage the transformation of the original conversation into a more reusable information artifact (i.e., content in the wiki repository). While the repository helps answer some questions, even its summarized and decontextualized write-ups are 
insufficient to answer all questions. However, it is still useful in helping to answer those difficult questions, because pages can be referred to in the discussion. Thus, question answerers need only recontextualize the wiki content to meet the specific needs of an individual, and can send the questioners to a wiki page for the details. This works well because question askers, who typically know the least about the topic, do not need to decontextualize the original messages to try and figure out which ones fit their own situation.

Although the reuse of information is facilitated by a wiki repository, it still requires a fair amount of effort to create a wiki repository. In the groups studied in this dissertation, this work was performed manually with little technical support. However, it would be possible to provide tools to help with the work of summarization and decontextualization. This approach is similar to Ackerman et al., (2003), but would be done for the entire community, not a single individual or small group of individuals. For example, an automated system could potentially recognize frequently discussed topics that are not yet in the wiki repository. These could be sent to an automatically identified expert with the recommendation that she create a wiki page on the topic, perhaps using the pre-identified messages as a starting point. Alternatively, messages that relate to a particular wiki page could be automatically identified or submitted by participants to be appended to the bottom of a related wiki page. The wiki page could include the basic information on the topic, which would help novices be able to decontextualize the actual appended messages and reapply them to their own situation.

\section{Methodological Considerations \& Suggestions}

This dissertation demonstrated the usefulness of conducting an empirical study, followed by an action research project. This combination was particularly useful in the current project because so little was previously known about the interplay between community conversation and wiki repositories. The study of css-d provided numerous 
practical insights and best practices that had been tried and tested in the field.

Furthermore, studying a technical support community enabled me to examine the use of a relatively novel technology before others had adopted it - allowing me to help transfer the experience to another domain before it would have otherwise occurred.

However, the css-d case study had its limitations. Although the selection of only one site allowed for a thorough examination of a community at a level of depth not possible with multiple sites, it also made the generalizability of the findings questionable. This was particularly problematic because one of the goals of the research was to devise practical suggestions to other communities hoping to augment their threaded conversation with a wiki repository. A related limitation was that a single case study (of a successful community) did not allow me to learn from the failed experiences of communities that attempted to adopt a wiki repository. I did learn from some unsuccessful practices attempted in the css-d community, but I could not answer questions about what types of communities are good (or poor) candidates for using a wiki repository.

The Medshelf action research project was able to address these limitations. The recruitment process (e.g., the feedback I received from participating and nonparticipating communities) helped uncover the most important issues related to adoption of a wiki repository. This provided insights into the types of communities that are well suited to use wiki repositories. Working with several communities on implementing a wiki repository (using the best practices from the css-d study) allowed me to validate and refine those suggestions. Because the project occurred in the "real world" with actual communities, the findings have high external validity. Also, because I chose communities that were sufficiently different than the field study community (i.e., medical rather than technical support communities), I was able to show that the successful suggestions had fairly wide application. This approach was also more risky, in that the chance of failure was greater - especially considering the unique challenges to medical support communities. However, it worked well in this case, where the communities I worked with 
ranged from very successful, to moderately successful, to unsuccessful. Although I had hoped all would be successful, I was able to learn from all of them including the one that did not work out. In hindsight, I believe that the repository was useful in such different domains because wiki repositories are so flexible and can be tailored to meet the specific needs of different communities. Thus, when transferring technologies that worked in one context to another it is important to transfer technologies that are sufficiently flexible and be open to the fact that other communities may use them differently.

One of the limitations of the approach I have described is that it does not lend itself as well to revolutionary designs. In order to validate some of the best practices from the earlier study I wanted to suggest the same ones to the new communities. I did manage to introduce some new ideas (e.g., the Wiki Digest, genre examples), although they were largely unsuccessful - at least in the early stages of the wiki (see Chapter 9). This reaffirmed to me the value of basing the majority of my suggestions on practices that had already worked in another community. Although future research can test more novel design ideas, I believe the approach I took led to stronger findings that were necessary as a basis on which to build. Had I tested more radical design ideas I would not have been able to validate and refine the original findings from the field study.

\section{The New Challenge of Mass Collaborative Authoring}

Although their ubiquity makes it easy to take online support communities for granted, they are quite amazing social structures that bless the lives of their members in a number of ways ranging from the provision of much needed information to the provision of social support and friendship. This is all accomplished by distributed volunteers who often have no prior relationships with other members. Threaded conversation has proved itself remarkably versatile in meeting the needs of a host of different online communities. This is likely because it matches so well with an activity that we are so intimately familiar with in face to face conversation. Although an entire community is participating 
in threaded conversations, any given thread is simply a conversation like hundreds of others occurring among a few people. The community benefits come from the fact that so many people are listening in (and jumping in) on the conversation at any given time.

Authoring documents is another activity that is common. However, it is not as innately collaborative as discussion. Although at rare times I find myself conversing with myself, it is certainly not the norm. However, authoring is very often an individual endeavor, and rarely an endeavor of more than a handful of people. This suggests that mass collaborative authoring, as occurs in wiki repositories and other sites like Wikipedia, is a relatively new activity with no close corollary in our daily lives. If this is the case, we as a people must develop social norms and practices around collaborative authoring to make it work effectively.

In addition to mass collaborative authoring's novely, there are a few social challenges that I identified in this dissertation, which warrant further exploration. One challenge is overcoming the "tragedy of the commons" where individuals are less likely to contribute to a shared good than would be optimal for the entire group. Getting others to contribute to the wiki repositories was a challenge for all communities, but only debilitating for one of the Medshelf communities. It was overcome at css-d because the benefits of contribution were helpful to the contributors (e.g., they could include links to wiki pages to make answering questions easier). In addition, some members contributed because those in authority recommended that they do so (often in public). Reciprocity was also a motivator at css-d and some Medshelf communities, as members contributed in order to give back to the community for helping them.

I also identified another less well recognized challenge to collaborative authoring: the hesitancy of members to edit and delete other members’ work. For example, I found that while deleting content is technically easier than adding content, it is socially far more challenging. Adding content only requires an assurance that at least one person will benefit from the content. Deleteing content, however, requires knowledge that the content 
was not useful to each of the community members. I also found that wiki content that is attributable to an individual (e.g., content directly copied from a community email list message) is less likely to be edited by other members. Thus providing attribution to a statement may be helpful in establishing credibility, while reducing its quality since it will not likely be maintained in the future. Both of these examples suggest that communities could benefit from establishing strong norms and expectations about collaborative authoring behaviors, although what those norms are remains a topic of future research.

Although collaborative authoring can be done in many ways, this dissertation has demonstrated the value of linking the authoring to conversations in an existing social structure, and some of the specific ways of forging that link. More generally, collaborative authoring provides many new opportunities for peer groups to create resources of immense value. Empowering these peer communities through collaborative authoring and other collaborative endeavors will have an enormous impact on the world in which we live. 


\section{APPENDIX 1}

\section{CSS-D TIMELINE}

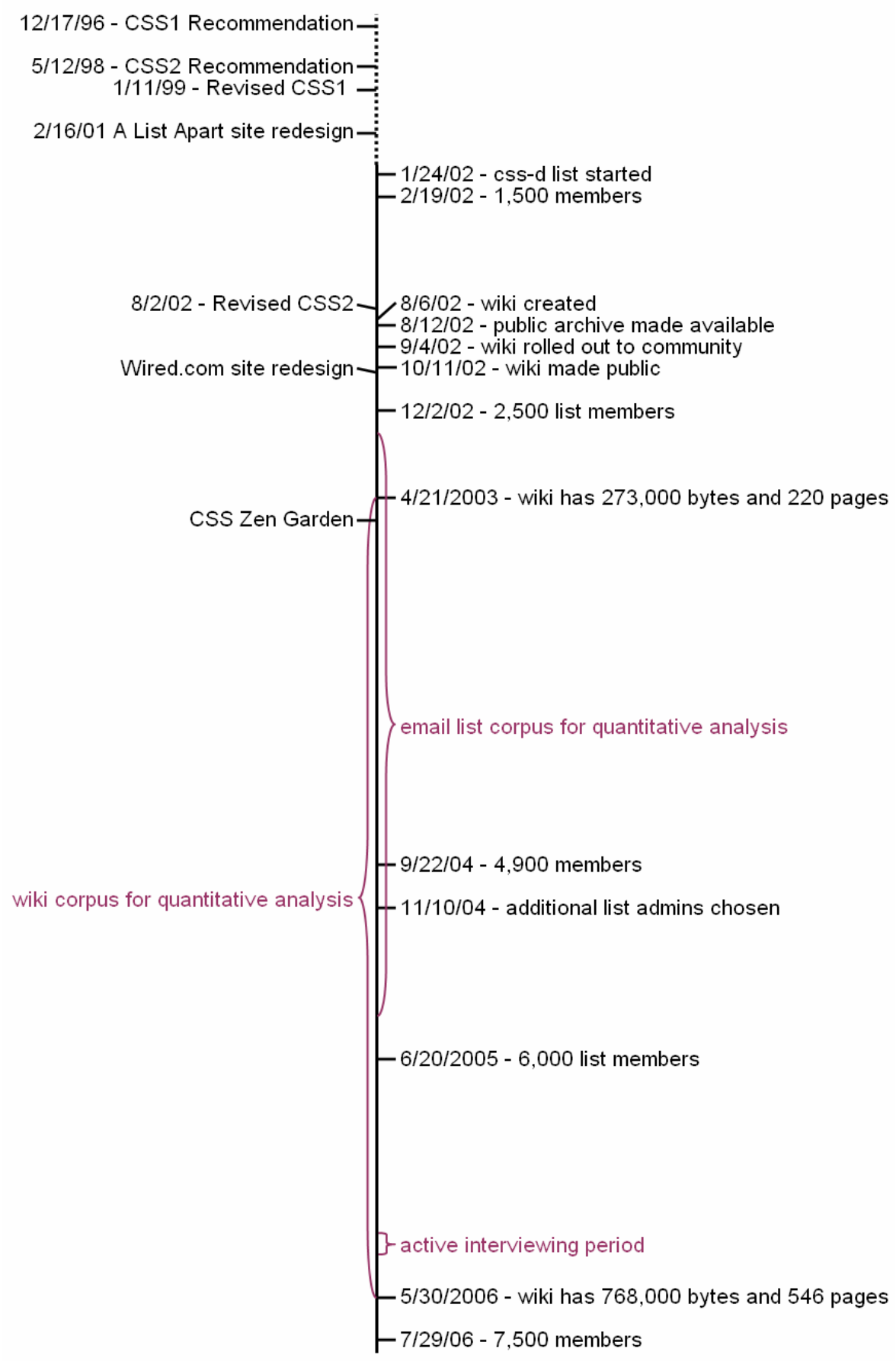




\section{APPENDIX 2}

\section{CSS-D CODE BOOK}

\section{A. CONTENT ANALYSIS OF REASONS FOR REFERENCING THE WIKI}

Note: each message can be categorized into multiple groups. Category names are in CAPITAL LETTERS below.

\section{Step 1. Verify that email in fact references the css-d wiki.}

MISTAKEN - Does not link to (or refer to) the css-d wiki. These include emails that use the word "wiki," but are talking about a different wiki (e.g., Wikipedia). Also includes messages where the only reference to the wiki was in quoted material (from a prior message) and the wiki content (or link) was not discussed in the "new" message content. A good rule of thumb is to ask the question "would the reply still make sense if the original message hadn't referenced the wiki?" If the answer is "yes," then it should be categorized as MISTAKEN, if it is "no" then it should not.

\section{Step 2. Tag each email with one or more reasons for referencing the wiki. Only content that is related to the wiki reference should be coded, NOT all of the email content!}

EDUCATE - Links to the wiki to help educate another user in reply to a prior message about a CSS or other web design topic (not about how to use the wiki). It may be to help answer a question, as an aside (e.g., "see also"), or part of a "site check" or

other activity. It also includes general pointers to the wiki for educational purposes (e.g., "there are probably many examples that you could find on the wiki”).

HELP ASK OR JUSTIFY A QUESTION - Refers to the wiki (or a wiki page) to justify a post (e.g., "I couldn't find anything in wiki on this") or to help explain the 
problem ("I'm using abc method found on wiki page P and am running into this problem").

META - Discusses what the wiki is and how it relates to the email list (e.g., please refer to wiki page when answering related questions), problems or good things about the wiki (including its appearance), and the general process of wiki contributions (e.g., "it's a wiki, feel free to edit"). Not a discussion of specific wiki content, where to find the wiki, or usability issues related to the wiki.

ADMIN - Helps shut down or avoid a holy war, keep the discussion on-topic, or explain list procedures and best practices and how to use the wiki technology and where to find it. The following subcategories should be used.

OFFLOAD DISCUSSION - Sends someone to the wiki to help end an off-topic discussion (after it has started) or to prevent one.

SEARCH WIKI BEFORE POSTING - A question answerer tells the question asker that they should look in the wiki BEFORE posting to the list. This does not include general comments that say "I recommend you look at the wiki". This can include implied comments if very obvious.

OTHER LIST PROCEDURES - Explains or encourages others to follow list procedures not related to the wiki (e.g., trim posts, reply-to default setting, how to subscribe and unsubscribe).

WIKI LOGISTICS - Questions and answers about how to use the wiki technology (e.g., how to make edits, why words are SmashedTogether) OR where it is found. Also includes comments from people about not being able to use the wiki (too difficult or couldn't figure it out) - but NOT problems with wiki content (see next category) or the social processes related to the wiki (which should be classified under META). Includes comments about passwords and other technical methods to avoid wiki spam. If people simply link to the wiki and say "it can be found here", then it is not enough to qualify for this section. Someone must ask specifically where it is or be responding to someone's 
direct question about it. Includes messages where there is confusion about how to use the Site Check system, or general complaints about it.

DISCUSS WIKI CONTENT EDITS (rule of thumb: things that would fit well on a Wikipedia talk page). The following subcategories should be used:

FUTURE OR POTENTIAL EDITS - Suggests (or proposes) new content be added to wiki OR encourages someone to add something to the wiki (e.g., "you should add that message to the wiki"; "You should name it XYZ", "I'm going to create a page on this topic so that people won't discuss it on the list", "If you give me a good response I will create page Q"). This may include implicit suggestions for future edits (e.g., "There seems to be a problem with page Z, is that right?", "the QYE link on page YZ is broken") if it is clear that the discussion is focused on the wiki content. Comments like "I couldn't find anything in the wiki, or on page Q" and "i'm using the method on page Y but it's not working like I expected" should not be classified in this group, they should be classified as HELP ASK OR JUSTIFY A QUESTION.

PRIOR WIKI EDITS - Discusses a prior wiki edit made by the person who posted the message or somebody else (e.g., "I just created page Z"; "I just removed spam"; "It looks like so and so added a bunch of pages"). Should not include messages that are brought up to "EDUCATE" if solely for the purpose of giving attribution to the original page author/editor (e.g., "You should visit the great page created by John Smith on that topic”).

THANK YOU - “thank you messages” that directly relate to wiki content (if ambiguous then still use this code). Should also include messages that say things like "I will definitely validate my css from now on" if a wiki page on validating css was sent to them. Should not include "thank you" messages that specifically thank the person for non-wiki related suggestions (those should be classified as MISTAKEN).

OTHER - Anything that doesn’t fit into at least one of the other categories (e.g., references to the wiki in order to support a particular argument). 


\section{B. CONTENT ANALYSIS OF REASONS FOR REFERENCING THE ARCHIVE}

Follow same procedure as with wiki message coding, but with categories outlined below.

\section{Step 1. Verify that email in fact references the css-d archives (either public or private).}

MISTAKEN - Does not link to (or refer to) the css-d public or private archives. These include emails that use the word “archive,” but are talking about a different archive. Also includes messages where the only reference to the archive was in quoted material (from a prior message) and the archive content (or link) was not discussed in the "new" message content. A good rule of thumb is to ask the question "would the reply still make sense if the original message hadn't referenced the archive?" If the answer is "yes," then it should be categorized as MISTAKEN, if it is "no" then it should not.

\section{Step 2. Tag each email with one or more reasons for referencing the archive. Only content that is related to the archive reference should be coded, NOT all of the email content!}

EDUCATE - Links to the archive to help educate another user in reply to a prior message about a CSS or other web design topic (not about how to use the archive). It may be to help answer a question, as an aside (e.g., “see also”), or part of a "site check” or other activity. It also includes general pointers to the archive for educational purposes (e.g., "there are probably many examples that you could find in the archive”).

HELP ASK OR JUSTIFY A QUESTION - Refers to the archive (or a specific archive message) to justify a post (e.g., "I couldn't find anything in the archive on this") or to help explain the problem ("I'm using abc method discussed in the archive at... and am running into this problem"). Also if people say "you can just send me a link to the archive if it's already been discussed."

META - Discusses what the archive is and how it relates to the email list and wiki (e.g., can we archive this list on gmane or some other service) OR talks about how 
to use the archive more effectively (not it's interface or specific search functionality though) - for example, using meaningful subject headers, including css snippets in messages instead of links that will be broken later. Also, discussion about using up archive space etc. should be included. Not a discussion of specific archive content, where to find the archive, or usability issues related to the archive.

ADMIN - Helps shut down or avoid a holy war, keep the discussion on-topic, or explain list procedures and best practices and how to use the archive technology and where to find it.

OFFLOAD DISCUSSION - Sends someone to the archive to help end an offtopic discussion (after it has started) or to prevent one.

SEARCH WIKI BEFORE POSTING - A question answerer tells the question asker that they should look in the archive BEFORE posting to the list. This does not include general comments that say "I recommend you look at the archives". This can include implied comments if very obvious.

OTHER LIST PROCEDURES - Explains or encourages others to follow list procedures not covered in the other categories (e.g., trim posts, reply-to default setting, how to subscribe and unsubscribe...).

ARCHIVE LOGISTICS - Questions and answers about how to use the archive technology (e.g., how to perform searches on it) OR where it is found. Also includes comments from people about not being able to use the archive (e.g., it's search functionality is broken). If people simply link to the archive and say "it can be found here," then it is not enough to qualify for this section. Someone must ask specifically where it is or be responding to someone's direct question about it. Includes messages where the archive is temporarily unavailable.

THANK YOU - "thank you messages" that directly relate to archive content (if ambiguous then still use this code). Should also include messages that say things like "I will definitely validate my css from now on" if an archived message on validating css 
was sent to them. Should not include "thank you" messages that specifically thank the person for non-archive related suggestions (those should be classified as MISTAKEN).

PAST CONVERSATION - Links the current message to a prior message (or thread) in order to draw attention to the continuation of the conversation (not to EDUCATE or HELP ASK OR JUSTIFY A QUESTION). For example, "there is a chart alluded to here last week - archive link"; "following up on the prior conversation...", "thanks to those who replied to my earlier message - archive link - here are the results..." OTHER - Anything that doesn’t fit into at least one of the other categories (e.g., references to the archive in order to support a particular argument). 


\section{APPENDIX 3 \\ MEDSHELF.ORG STARTUP MATERIALS}

\section{Main Page}

This page is not yet ready to be made public. Smaller communities should read the Project:Simple Start page for ideas on getting your wiki up and running. Larger communities may want to see the extended version at the Project:Getting Started page. Make sure you delete this comment and customize this page once the wiki is ready to be introduced to the community.

Welcome to the [Community Name] Wiki! This wiki serves as the community's longterm memory and public outlet to the world. Please read our About page and Disclaimer and understand that the information found in this wiki is not provided by medical experts. We hope that you find the content useful and help improve it by making your own edits (see the Wiki 101 page for basic instructions). After all, it is a community project run by individuals like you!

\section{[Community Name]}

- [Community URL if it exists] - Our community website

- [Community Email List] - Information on how to join and unsubscribe from the email list

- [Email List Archives]

\section{Using the Wiki}

- First time wiki user? See our Wiki Guide

- Feeling experimental? Learn how to edit pages and then try editing the Sandbox.

- Share your recommendations on how to improve the wiki at the Suggestion Box or volunteer to become one of our Wiki Helpers.

- You can also view All Pages, Help Pages, or Special Pages.

\section{[Community Topic] Information}

This section serves as an index to the Default Wiki's contents. 


\section{Subtopic 1}

\section{Subtopic 2}

\section{Project:Simple Start}

Welcome to your community wiki! This page includes some suggestions to help you get your wiki up and running. The suggestions are designed for small communities with a single person that will head up the project. The general idea is to get things started off on the right foot without much effort, and then (with the help of the community) slowly grow the wiki into a more comprehensive resource.

If you represent a larger community and have several people who can work on the wiki from the start, you may want to view the Getting Started page instead of this one.

So, here are our suggestions:

\section{Announce the Wiki to your Community}

The first step is to introduce the wiki to your existing community and ask for their involvement with it. You may want to use our Sample Introduction Message, although you'll need to customize it to your particular community. The main purpose of the message is to let members know what the wiki is, get them excited about the project, find out if they have any concerns, and ask for their help. It is also a way of determining who may be interested in helping you add content to the wiki (see next section)

\section{Seed the Wiki with Content}

One thing you don't want to do is send everyone to an empty Wiki. They will likely be turned off from the start if it is a completely barren wasteland. So, before you invite the community to see the new community wiki, make sure and create a few high quality pages on-topics of general interest. You probably already know the hot topics, but you may want to get ideas, and even content, from others (e.g., see the Sample Introduction Message). Encourage other enthusiastic members to help you seed the wiki by creating new pages or improving the ones you've created. Instead of writing everything yourself, summarize or copy (with permission) what others have already written in the email list or forum. Just make sure you don't include people's names where they don't want them included. 


\section{Learning to Use the Wiki}

Before adding content you will have to learn how to use the wiki. There are many advanced features (all of which are described in the help pages), but you should start with the basics as described in the Wiki Guide, Help:Wiki 101, and Help:Editing pages. A good way to start is to create an account and then put some content in your user page (see User:DerekHansen for an example). Once you understand editing, you may want to think about how to organize pages (see Help:Organizing pages). You will also play the role of Administrator, so you should be familiar with the administration features at some point. But remember, you only need to learn a piece at a time.

\section{Deciding What to Include}

What type of information should be included? The wiki is not meant to replace the email list or forum conversation. Instead, it is meant to supplement it. The email list or forum is a conversation, while the wiki is a collection of resources. The wiki includes summarized or distilled information of lasting value. The email list or forum discussion is all about taking care of each other's immediate needs. The wiki content, on the other hand, is applicable to many people. So, whatever topics you create pages for, make sure that the content is applicable to many readers and in a summarized form. Remember, you also don't want to re-create the wheel. If there is another web page that covers a topic well, just link to the page and explain why you like it. In fact, some of your most useful wiki pages may include mostly links.

Once you've created the initial pages, make sure you edit the Main Page so that it links to each of your pages. That way visitors to the site will be able to browse through all of the pages.

\section{Update Existing Pages}

Below is a list of existing pages that will require your attention before rolling out the wiki:

- Project:About: Needs a brief description of your community.

- Main Page: Needs to have introductory paragraph in italics deleted and links to pages that you have created added to the bottom section.

- Wiki Guide: Add your community name where indicated.

- Wiki Team: Add usernames of Wiki Team members and contact information for the group. When you start you may only have one "Wiki Team" member, but over time others can come onboard.

- Wiki Helpers: Add usernames where appropriate. Again, this may just be you to start with. 


\section{Advanced Suggestion:}

You may want to look at the Wiki Genres or Sample Main Page for ideas on topics that you may want to include, although they would need to be tailored to your specific community.

\section{Promote the Wiki}

The real benefit of a wiki over a standard website is that it can be a truly collaborative effort, rather than the work of an individual or small team. The idea is that your community will take ownership of the wiki and turn it into the type of resource that will be of most value to them. But, this generally requires some coaching on your part. Here are a few ideas of how to get others involved.

\section{Initial Activities}

- Post to the community to let them know that the wiki is now public and ready for their participation. See Project:Sample Roll Out Email for ideas.

- Link to the wiki from other highly rated webpages so that it will show up better in search results. If you have a community website already, make sure and link to the wiki from it.

- Put a link to the wiki in the email list or forum message footer if possible.

\section{Ongoing Activities}

- Encourage community members to reference specific wiki pages in the ongoing conversation (e.g., on the email list or forum). When specific pages are referenced in messages, people are more likely to keep up on what pages are available. They are also more likely to improve those pages themselves. Here are some suggested ways to help this happen:

o Lead by example. When you are answering a question, include a reference to relevant wiki pages. When you ask a question, mention what pages you have already looked at. When new members post, let them know that exploring the wiki would be a good idea and point to a couple of relevant pages. When you create a new page or make a big change, announce it on the community and ask for others' contributions. When you are preparing a new wiki page, post to the community and ask them for ideas on what should go in it. When you are worried about a discussion becoming contentious or off topic, link to a relevant wiki page (e.g., a Project:Comparison Genre page or policy page) to help offload the discussion.

o Treat the wiki as a supplement to the discussion, not a replacement. Many activities such as providing social support, helping identify 
problems, and tailoring information to an individual's specific needs are best done through the ongoing conversation. In these situations, linking to wiki pages is an easy way to provide additional resources that will allow the reader to explore more on their own. Community members should not simply send others to the wiki for answers. Instead, they should give a customized answer and then identify specific wiki pages that may be pertinent to them.

- Encourage community members to contribute to the wiki themselves. The wiki will best succeed if it is a community activity, rather than the work of an individual or even the Wiki Team. Here are a few ideas on how to encourage widespread participation in the wiki.

o Identify specific ways that community members can help. Encourage community members to put the content from a particularly helpful message onto the wiki, or add it yourself and let them know so they can update or improve it. Single out individuals with specific expertise and ask them to contribute to a specific page. When a frequent question arises, ask for a volunteer to summarize the discussion into a wiki page. Ask if members have ideas for new pages and if they do, ask for their help in creating them.

o Help members make at least one edit. After that, they will be much more likely to make more edits. Offer extra encouragement and help to them their first time. You may even suggest that all members make a simple edit just to get them started (e.g., add their name to an existing page).

- Give public praise of wiki contributions. This can be done in the ongoing email list or forum discussion, as well as in a Wiki Digest (if you get ambitious).

- Emphasize that all contributions are welcome. Contributions to the wiki need not be perfect, since others will edit them.

\section{Project:Getting Started}

This page is for those of you getting your community wiki up and running. It tells you what you will need to do and what decisions need to be made before rolling out your wiki to the entire community. It also includes recommendations on best practices based upon prior research. This page should be used to help fill out the Project:Checklist page, where you can keep track of each decision and finished task. If you are not yet familiar with the wiki, you may want to first read the Wiki Guide which explains what the wiki is and how it can be used.

\section{Announce the Wiki to Your Community}

The first step is to introduce the wiki to your existing community and ask for their involvement with it. You may want to use our Sample Introduction Message, although 
you'll need to customize it to your particular community. The main purpose of the message is to let members know what the wiki is, get them excited about the project, find out if they have any concerns, and ask for their help. It is also a way of determining who may be interested in joining the Wiki Team (see next section).

Once you have sent out the introduction message, make sure to update the appropriate Checklist section.

\section{Establish a Wiki Team}

\section{Create the Wiki Team}

The next step is to create a small group of community members who will take ownership of the wiki. This group will provide the initial content and encourage other community members to use the wiki on an ongoing basis. Team members can be first-time wiki users, but should be willing to spend a little time getting familiar with the technology. Here are a few suggestions related to the Wiki Team

- The team should include a handful of individuals. Too few members doesn't allow for enough different ideas to surface. Too many makes it difficult to coordinate and get things done efficiently. More diversity of experience (e.g., newly diagnosed members, long-time experts) is preferred.

- The team should frequently bounce ideas off one another and try to make major decisions together. You may want to create an email list for the Wiki Team that can be used to communicate with one another and used by others to contact the entire team.

- Many teams find it useful to rotate members on occasion to bring in new ideas and avoid burnout.

\section{Determine Member Roles}

Once your Wiki Team is established, you can discuss what specific community roles each member would like to fill. Some individuals may play multiple roles, especially for smaller communities. Many of these roles are described on the Wiki Helpers page. Here are a few suggested roles for Wiki Team members:

- Wiki Team Leader: This individual oversees the Wiki Team and is the primary contact person for the MedShelf.org research team. They post messages to the community email list or forum on behalf of the Wiki Team and help initially recruit Wiki Team members. They have "Bureaucrat" status, which means they can make others "Administrators" so that they have the ability to delete pages, protect pages, and block users (see Help:Administration for details).

- Clean Up Crew Leader: This individual oversees the Clean Up Crew by performing the clean up activities regularly and by recruiting community 
members to join the crew and help maintain the content. They decide if Clean Up Crew members are trustworthy enough to give them "Administrator" privileges so they can delete pages. They also raise important Clean Up Crew issues with the Wiki Team.

- Promotion Crew Leader: This individual oversees the Promotion Crew by performing the promotion crew activities regularly and by recruiting community members to join the crew. The also raise important Promotion Crew issues with the Wiki Team.

- Main Page Advocate: This individual plays the role of a Page Advocate for the Main Page. The Main Page is especially important since it is the first page anyone visits and is an organized index to the rest of the wiki content. For this reason, a Wiki Team member may want to take some ownership of it. This individual reviews others edits of the page and raises important issues related to the page with the Wiki Team. They may want to protect the page from others edits if people misuse it. The Project:Sample_Main_Page provides some guidance on how to structure the actual Main Page.

- Wiki Digest Chief Editor: This individual oversees the Wiki Digest. The Wiki Digest is like a brief newsletter about the wiki sent to the email list or forum on a regular basis. It may be the work of one individual (the Chief Editor) or several individuals that are part of the Wiki Digest Editors group. The Chief Editor posts the digest to the list, helps develop its content, coordinates other editors, and raises important issues with the Wiki Team. The Project:Sample Wiki Digest is an example of what a finished Wiki Digest may end up looking like.

Once you have decided on the community roles of the Wiki Team members, make sure to update the Wiki Team and Wiki Helpers pages to reflect the changes and fill in the appropriate Checklist section.

\section{Decisions}

\section{Who can contribute to the wiki?}

Several options are available. Here are the main ones.

- Option 1: Anyone (i.e., registered users and anonymous contributors). Anonymous posters are identified by their IP address when they contribute, while registered users are identified by their username. This is the default option.

- Option 2: Registered users only (i.e., only people who have registered themselves and are currently logged in; no anonymous contributors).

- Option 3: Registered, pre-approved users (i.e., only people who the Wiki Team has approved and allowed to register). This requires that the Wiki Team add new users to a pre-approved list before they are able to edit the wiki. This can be done by having people email the Wiki Team with a preferred username and password and then adding the usernames to a list of allowed users. 
Here are a few things to consider:

- Privacy. None of the options above allows for completely anonymous edits of the wiki. This helps prevent vandalism of the site, but also doesn't allow people to post information that they would not want tracked to them. Rather than promise complete privacy through anonymity, we suggest that people limit their contributions to things that they would not mind being traced to them. Having said this, each of the above options makes it relatively easier or harder to tie a particular comment to an individual. Option 1 allows people to post semianonymously by only showing IP addresses of contributors that are not logged in. IP addresses are relatively difficult to trace to an individual, although in some cases it can be done. Option 2 allows people to create a username that is unrelated to their real name (if desired), making it difficult for people to link comments to an "offline" individual. Option 3 also allows members to create their own usernames but requires that the Wiki Team know who each person is.

- Encouraging Participation. The harder it is to participate, the less likely it is that people will actually contribute to the wiki. Option 1 requires very little from a potential editor, while option 3 requires quite a bit. Choosing option 1 will likely encourage more community involvement, but may also lead to problems with "wiki spam". To help reduce spam, administrators can exclude IP addresses of vandalizers from posting messages as described in the Help:Administration page.

- Credibility. In order to determine the credibility of information it is often helpful to know the source of that information. Option 1 allows people to identify themselves when they want, while the other options require that they identify themselves (at least as a consistent online username).

- Changing Options. It is easy for the MedShelf.Org maintainers to change this setting. This makes it possible to begin with one option and switch to another if needs change. For example, a community could start by allowing anyone to post (which is the default option). If they find that there are abuses, they can then switch to one of the other options (by emailing Derek Hansen).

Once you have made your decision make sure and update the appropriate Checklist section.

\section{Seed the Wiki with Content}

Before opening the wiki up to the entire community, the Wiki Team should provide some initial content and the basic organization of the site. Here are a few suggestions related to seeding the wiki.

\section{Systematically Decide What Should be Included}

Two general categories of content are particularly important. First, content that is of widespread interest (e.g., topics related to frequently asked question). And second, 
valuable content that is not readily available elsewhere. To help decide what should be included you may want to...

- Read through prior community messages (e.g., email list archives or forum messages) looking for frequently discussed topics or messages that are particularly well-written, informative, and unique. Content from these messages can often be copied or summarized into Wiki pages, lessening the workload of the Wiki Team. If copying directly from past messages you will need to decide if (and how) you will give credit. Some individuals may want credit, while others will not want their name publicly associated with the content. One safe approach to dealing with this issue is to give general credit to the "list members" and allow list members that want their name associated with specific content to add their name later. Another approach is to provide a link to the original archived message that may only be visible to registered email list or forum members.

- Ask the community to recommend topics. This is already part of our Sample Intro Message. If you didn't receive a great response, you may want to solicit ideas in a separate message to the community or directly from a few knowledgeable members.

- Discuss with the Wiki Team how the content in this wiki will differ from other resources already available. You may want to refresh your memory on what resources are already available online before having this discussion. What unique niche will the wiki fill? How can it complement existing web resources and help fill in the gaps? The idea of the wiki should not be to recreate the wheel. Instead, it will be most valuable if it helps identify good existing resources (by linking to them) and fills in the gaps.

\section{Create Good Content in a Useful Format}

Make each finished page look as professional, complete, and high quality as you can. Later contributors will likely follow the example of existing pages, so set a good one. Also, some people may be more willing to contribute if they respect the existing pages. Rather than creating a lot of half-finished pages, it is better to focus on creating a few high quality pages that will be useful right away. There will be plenty of time for you and others to add more pages later.

- Comply with copyright laws. Make sure that you do not copy works that have been copyrighted. When possible, simply link to the original source. If you rely heavily on a particular source, make sure you reference it.

- Consider what Wiki Genres should be used to present the content. A Wiki Genre is like a template. It can be used to help organize a page. It helps other contributors know what types of information should be added and what tone to use. It also helps readers know what to expect. A collection of generic Wiki Genres and ideas on when to use them can be found at the Wiki Genres page. You can also see the Sample Main Page for ideas about potential wiki pages and Wiki Genres that support them well. 


\section{Organize Your Content}

Because wikis are always changing, it is important to consider how people will find content that they are looking for. Although they can search or see a list of All Pages, it is often helpful to have content organized so that people can browse certain topics. See Help:Organizing pages for more details. Here are a few ideas on how to start organizing the content.

- Use the Main Page as an organized index to the wiki content. Each time you create a new page, you should add it to an appropriate section of the Main Page or make sure that you can get to it from another existing page. The Sample Main Page will give you an idea of what a completed Main Page looks like.

- When you have many related pages, create a single index page that links to the others. For example, you could have a page called Definitions that includes an alphabetized list of medical terms. Each medical term could have its own page (e.g., the uritis page would provide a definition of uritis). The Definitions page would serve as an index to the specific pages with the actual content on them. This way, when the term "uritis" is used on other wiki pages, it can link to the uritis page that contains the definition, not the entire list of definitions on the Definition page. This also allows you to link to the Definitions page from the Main Page instead of cluttering up the Main Page with a link to each individual definition page (e.g., uritis).

- Provide links to related pages. If you have a page that is directly related to other wiki pages, make sure you link to those pages either within the text or in a separate "Related Pages" section of the page.

- Make sure you don't have any Orphaned Pages (i.e., pages that are not linked to from any other pages).

\section{Update Existing Pages}

Below is a list of existing pages that will require your attention before rolling out the wiki:

- $\quad$ Project:About: Needs a brief description of your community.

- $\quad$ Main Page: Needs to be customized. See the Project:Sample Main Page for ideas on what it will look like when completed.

- Wiki Guide: Add your community name where indicated.

- $\quad$ Wiki Team: Add usernames of Wiki Team members and contact information for the group.

- Wiki Helpers: Add usernames where appropriate.

- $\quad$ Project:Copyrights: Modify license if not using recommended one.

As you seed the wiki with content, make sure to update the appropriate Checklist section. 


\section{Promote the Wiki}

The success of the wiki will largely depend on how well it is promoted to community members and the world at large. Below are a few suggestions relating to the promotion of the wiki.

\section{Initial Activities}

- Post to the community to let them know that the wiki is now public and ready for their participation. See Project:Sample Roll Out Email for ideas.

- Link to the wiki from other highly rated webpages so that it will show up better in search results. If you have a community website already, make sure and link to the wiki from it.

- Put a link to the wiki in the email list or forum message footer if possible.

\section{Ongoing Activities}

- Send out a regular Wiki Digest message to the community. It could spotlight individuals who have contributed, highlight new or exceptional content, request new pages, recommend a page in need of review by the community, discuss policies or decisions made by the Wiki Team, provide a tip for new wiki users, and ask for suggestions. The Sample Wiki Digest is an example of what a finished Wiki Digest may end up looking like.

- Encourage community members to reference specific wiki pages in the ongoing conversation (e.g., on the email list or forum). When specific pages are referenced in messages, people are more likely to keep up on what pages are available. They are also more likely to improve those pages themselves. Here are some suggested ways to help this happen:

o Lead by example. When you are answering a question, include a reference to relevant wiki pages. When you ask a question, mention what pages you have already looked at. When new members post, let them know that exploring the wiki would be a good idea and point to a couple of relevant pages. When you create a new page or make a big change, announce it on the community and ask for others' contributions. When you are preparing a new wiki page, post to the community and ask them for ideas on what should go in it. When you are worried about a discussion becoming contentious or off topic, link to a relevant wiki page (e.g., a Project:Comparison Genre page or policy page) to help offload the discussion.

o Treat the wiki as a supplement to the discussion, not a replacement. Many activities such as providing social support, helping identify problems, and tailoring information to an individual's specific needs are best done through the ongoing conversation. In these situations, linking to wiki pages is an easy way to provide additional resources that will allow 
the reader to explore more on their own. Community members should not simply send others to the wiki for answers. Instead, they should give a customized answer and then identify specific wiki pages that may be pertinent to them.

- Encourage community members to contribute to the wiki themselves. The wiki will best succeed if it is a community activity, rather than the work of an individual or even the Wiki Team. Here are a few ideas on how to encourage widespread participation in the wiki.

o Identify specific ways that community members can help. Encourage community members to put the content from a particularly helpful message onto the wiki, or add it yourself and let them know so they can update or improve it. Single out individuals with specific expertise and ask them to contribute to a specific page. When a frequent question arises, ask for a volunteer to summarize the discussion into a wiki page. Ask if members have ideas for new pages and if they do, ask for their help in creating them.

o Help members make at least one edit. After that, they will be much more likely to make more edits. Offer extra encouragement and help to them their first time. You may even suggest that all members make a simple edit just to get them started (e.g., add their name to an existing page).

o Give public praise of wiki contributions. This can be done in ongoing email or forum discussions, as well as in the Wiki Digest.

o Emphasize that all contributions are welcome. Contributions to the wiki need not be perfect, since others will edit them.

As you complete some of the initial recommendations in this section, make sure to update the appropriate Checklist section.

\section{Project:Checklist}

This page is designed to help the Wiki Team keep track of the tasks they have completed and decisions they have made. Please refer to the Project:Getting Started page for suggestions and detailed explanations. You will need to edit this page in order to update it. You may want to practice editing the Sandbox before editing this page.

\section{Filling out this Checklist}

\section{Tasks}

Once you have completed a task, just add the text "(done)" to the beginning of the line. For example: 
- Task 0: Read through the Project:Getting Started page.

will read...

- Task 0 (done): Read through the Project:Getting Started page.

\section{Decisions}

Once you have made a decision, make the chosen decision bold and leave the others as regular text. Don't delete them, in case you want to change the decision at a later point. For example:

Decision 0:

- Wear Red

- Wear Blue

- Wear Black

indicates that "Wear Blue" is the chosen decision.

\section{Step A: Announce the Wiki to Your Community}

See related section on Project:Getting Started

- Task 1: Send a message to the list describing the Wiki. See Project:Sample Intro Email for ideas.

\section{Step B: Establish a Wiki Team}

See related section on Project:Getting Started

- Task 1: Recruite a handful of Wiki Team members.

- Task 2: Create a mailing list for the Wiki Team.

- Task 3: Have each member create an account on the wiki (see Help:logging in)

- Task 4: Give Wiki Team members Administrator rights (see Help:Administration for details)

- Task 5: Discuss the Wiki Helpers page and update the page so that at least one member of the Wiki Team is listed as the leader of the Clean Up Crew, Promotion Crew, and Wiki Digest Editors.

- Task 6: Update the Wiki Team page so that it includes the usernames of the Wiki Team members and any special roles they fill (e.g., Clean Up Crew leader, Wiki Promotion leader, Wiki Digest editor). 


\section{Step C: Decisions}

See related section on Project:Getting Started. Note: the default options are in bold when you first read this page.

\section{Decision 1: Who can Contribute to the Wiki?}

- Option 1: Anyone (i.e., registered users and anonymous contributors).

- Option 2: Registered users (i.e., only people who have registered themselves and are currently logged in; no anonymous contributors).

- Option 3: Registered and pre-approved users (i.e., only people who the Wiki Team has approved and allowed to register).

Task 1: Email Derek Hansen at shakmatt at umich dot edu with your decision so he can configure the software appropriately.

\section{Decision 2: What Creative Commons License should be used?}

- Option 1: Attribution alone (by)

- Option 2: Attribution + Noncommercial (by-nc)

- Option 3: Attribution + NoDerivs (by-nd)

- Option 4: Attribution + ShareAlike (by-sa)

- Option 5: Attribution + Noncommercial + NoDerivs (by-nc-nd)

- Option 6: Attribution + Noncommercial + ShareAlike (by-nc-sa)

Task 2: If you choose a license other than Option 1: Attribution alone (by), update the Creative Commons License section of the Project:Copyrights page.

\section{Step D: Seed the Wiki with Content}

\section{See related section on Project:Getting Started}

- $\quad$ Task 1: Read through prior community messages (e.g., email archives or forum messages) looking for ideas of what content should be included.

- Task 2: Collect ideas from members of the community. If you didn't receive any from the Sample Intro Message you may want to solicit ideas in a separate message to the list or from a few knowledgeable members directly.

- Task 3: Discuss with the Wiki Team how the content in this wiki will differ from other resources already available. What unique niche will it fill? How can it complement existing web resources and help fill in the gaps?

- $\quad$ Task 4: Decide what Wiki Genres you want to use for the various topics. Create your own or mix them together if it is helpful. See the Sample Main Page for ideas on possible topics and Wiki Genres that support them. 
- Task 5: Create new pages and update the Main Page so it is an organized index to the pages or sections of the wiki. You can use the Sample Main Page for ideas.

- Task 6: Make sure the following existing pages are updated appropriately (e.g., replace "[insert community name]" with your actual community name).

o Project:About: Needs a brief description of your community.

o Wiki_Guide: Add your community name where indicated.

- Task 7: Visit the All Pages page. Double check that each page in the "Main" namespace is complete. Make sure they are organized appropriately and that you can access each page from at least one other page. (Hint: Orphaned Pages shows all pages that are not linked to by any other page).

\section{Step E: Promote the Wiki}

See related section on Project:Getting Started

- Task 1: Post a message to the community to let them know that the wiki is now public and ready for their participation. See Project:Sample Roll Out Email for ideas.

- $\quad$ Task 2: Link to the wiki from your existing community website(s) and other popular sites you have access to.

- Task 3: Put a link to the wiki in the email list or forum message footer.

- Task 4: Make sure to continue to perform the ongoing activities that will help promote the wiki on a regular basis.

\section{Wiki Guide}

Welcome to the Default wiki! This wiki is a shared resource created and maintained by people like you. This page will help get you started using the wiki if you are a first-time user.

\section{What is a wiki?}

A wiki is a website that can be edited by many people. It keeps a history of all of the changes, so you can edit pages without worrying about ruining them. It also lets people format text, link to other pages, and organize content by using a markup language. For example, to link to another wiki page you would type [[Main Page]] when editing the page and it would show up as Main Page when viewing the page.

This particular wiki is a collection of resources created by Default members and other interested individuals. It serves as a community memory and helps supplement the email list discussion. See About for more details. 


\section{How is this wiki related to the email list?}

The email list is where the ongoing community discussion occurs. It is ideal for asking questions and providing personalized answers, getting a second opinion, helping one another through difficult times, celebrating milestones, and announcing information of general interest.

The wiki, on the other hand, includes content of lasting value. Instead of being tailored for one individual, it is general enough to be of benefit to many. It can be thought of as a distilled version of the conversation, as well as a collection of resources that can be used by the community. Specific wiki pages are meant to stand on their own and may take on many forms (see Wiki Genres).

The email list and wiki work best when they are closely tied to one another. Each can support the other. The email list discussion helps wiki contributors know what topics are interesting and provides content that can be summarized and added to the wiki. The wiki content improves the email list discussion by helping members formulate questions and answers more effectively. For example, when answering a question a member may share a personalized answer and also include a specific link to a relevant wiki page for more information. The wiki also shares the community's knowledge more widely, increasing our reach. Because the email list and wiki complement each other, you are encouraged to reference the wiki in the email list discussion. You are also encouraged to use content from the email list in the wiki. When doing this make sure any personal information is removed and it is presented in a distilled or summarized manner.

\section{How can I contribute?}

There are many ways to contribute to this wiki. Here are a few:

- $\quad$ Edit existing pages. See a problem with a page or notice something is missing? Just click on the "Edit" tab in the upper right-hand side of the page. You may

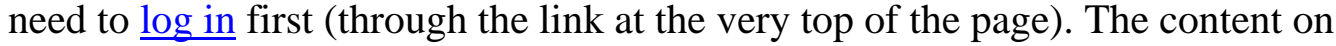
this wiki is shared by everyone, so don't worry that you are stepping on anyone's toes by editing material that they originally added. If others don't like your edits they can refine them. See Help:Wiki 101 for a simple editing example and Help:Editing for more details, then try out your new knowledge by editing the Sandbox page.

- Create a new page. See the Help:Starting a new page link to learn how to create a new page. Once you've created a new page you can let others know about it by emailing the page's URL to the email list and adding a link to it on the Main Page or some other page. Even if you can't finish the page, take a stab at starting it and letting others fix it up. You can even ask the email list members to help fill it in when you announce the page to them. 
- Adopt a page. Pick a page that is of particular interest to you and make sure it is kept up to date and of high quality. For more ideas on how to do this see Wiki Helpers\#Page_Advocates (i.e., the "Page Advocates" section found on the Wiki Helpers page).

- Refer to specific wiki pages in the email list. The more you point others to the wiki, the more they will benefit from its content and improve it themselves. This can be done by announcing new pages and major edits, bringing up troublesome pages, suggesting that someone transfer an insightful email thread to the wiki, asking for help with a particular page, pointing to pages that you found helpful in formulating a question, and referring others to specific wiki pages when answering a question (e.g., "for more info see..."). When using the wiki to help answer questions, make sure you don't treat the wiki as a substitute for good discussion. If you are answering a question on the email list, provide a personalized answer and point them to the wiki for more information instead of simply sending them to the wiki.

- Become a Wiki Helper. Once you become more experienced using the wiki you may want to join the Clean Up Crew, Wiki Team, or Promotion Crew. See Wiki Helpers for details.

- $\quad$ Suggest improvements to the wiki. Have an idea for a new page you would like created? Find a problem with the software? Drop a note in the Suggestion Box.

\section{Things to consider before contributing}

- This is a publicly readable wiki. If you don't want the whole world to know, then don't say it here.

- Edits are not entirely anonymous. Every time the wiki is edited, the username (if logged in) or computer's IP address (if not logged in) are associated with the edit in the Recent changes page, page histories, and user contribution pages. This helps us block out troublemakers (e.g., spammers) and allows other members to know who is posting content, but it also has privacy implications. If you don't want others to know that it is from you, then you may not want to post it here. You should also be wise in selecting a username if you are concerned about others tracking your comments to a real person. You do not need to select a username that is based on your real name or provide your real name when creating an account. You can be assured that we will follow the procedures outlined in the Privacy policy.

- You do not retain the copyright for your own contributions. If you want to retain the copyright for your own contributions you should post them to a personal website and link to it from this wiki. The copyright for material posted to this community's wiki is covered under a Creative Commons license (see Copyrights for details).

- This wiki is part of a research project. The wiki content will be analyzed as part of a research project. Please see the About page for details on the project and the ethical procedures that are followed by the researchers. 


\section{How can I learn to better use the wiki?}

The best way to learn how to use the Wiki is to start using it. You can practice wiki formatting by editing the Sandbox page. You can use the "Show Preview" button when editing a page to see your edits before they are submitted. Just make sure you click on the "Save page" button to make them final.

Once you are comfortable editing, try updating your personal user page. If you have registered and logged in, you will have a username. Your user page can be accessed by typing "User:your username" into the search box and clicking on the "Go" button. You will be taken to your user page (which is currently blank) and can click on the "edit this page" link to add content.

Remember, you don't need to be a wiki expert to contribute. Even if you only add plain text, others can format your material later. As you edit existing pages you will naturally learn more tricks from seeing how other people create bulleted lists, link to pages, etc.

Below are the most important help pages to get you started:

- Help:Wiki 101 - find out just how easy it is to edit the wiki

- Help:Logging in - establish your identity on the wiki

- Help:FAQ - some frequently asked questions about the wiki and how to use it

- Help:Editing - learn how to add formatting to pages (see here for more examples)

- Help:Starting a new page - learn how to create new pages and let others know about it

- Help:Searching - learn how to find the information you want

- Help:Recent changes - learn how to track edits to the wiki using the Recent changes page

- Help:Contents - the front page of the help section that will introduce more advanced topics such as...

o Help:Organizing pages - learn various techniques for organizing wiki pages

o Help:Images and other uploaded files - learn how to upload photos and insert them in pages

o Help:Namespace - learn why some page names start with different prefixes like "Help:" and "User:"

o Help:Preferences - customize the wiki by setting your user preferences

- Help:Renaming a page - learn how to rename a page and how the old page redirects to the new one

o Help:Watching pages - learn how to get email notification when pages you are "watching" are edited by others 


\section{Project:About}

\section{About [Community Name]}

[Insert brief description about your community]

\section{About MedShelf.org}

MedShelf.org is part of a research project conducted by students and faculty at the University of Michigan. The goal of the project is to help online medical support groups effectively use a wiki to collaboratively create useful resources. It includes content provided by members of several participating online medical support groups that is not reviewed by medical professionals (see Disclaimer).

If you represent an online medical support group that would like to participate in the study, please contact Derek Hansen (see contact information below).

\section{Research Project}

The research project is conducted by Derek Hansen, Paul Resnick, and Sean Munson of

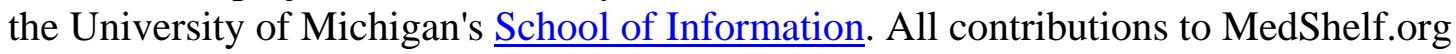
will be analyzed by the research team as part of the project. In addition, we will contact some contributors for voluntary interviews. The rights and privacy of individual members will be respected throughout. In conjunction with the University of Michigan's Institutional Review Board we have developed ethical guidelines to ensure your protection. Details of the study goals and the ethical procedures we are following are provided at this link.

\section{Contact Information}

If you have any questions or concerns about MedShelf.org or the associated research project, please don't hesitate to contact Derek Hansen via email at shakmatt at umich dot edu or via phone at 734-764-1008.

\section{Project:Copyrights}

This page describes the copyright information for contributors and those who wish to reuse content from the Default wiki found on Medshelf.org. It also describes the copyright licence that is used to cover the content. 


\section{Contributors' Rights and Obligations}

The content on this wiki is not owned by the individual contributors. It is covered under the Creative Commons license described (see below). This means that by contributing, members implicitly agree that the content they provide can be used as the license dictates.

Contributors to this wiki should comply with copyright laws. All works are copyrighted unless they either fall into the public domain or their copyright is explicitly disclaimed. Never use materials that infringe the copyrights of others. This could create legal liabilities and seriously hurt the project. If in doubt, write it yourself or link to the original source.

Note that copyright law governs the creative expression of ideas, not the ideas or information themselves. Therefore, it is legal to read an article or other work, reformulate the concepts in your own words, and submit it to the wiki. However, it would still be unethical (but not illegal) to do so without citing the original as a reference. See Wikipedia's articles on plagiarism and fair use for discussions of how much reformulation is necessary in a general context. Also note that the copyright policy of this wiki is different than Wikipedia's copyrights policy which is covered under a difference license, although content from their policy was used to inform this policy.

\section{Reusers' Rights and Obligations}

You are free to use all content from Medshelf.org in your own books, articles, web sites, or other publications, as long as you comply with copyright license. Please note that there are many specific community wikis on MedShelf.org, each of which may use a different version of the Creative Common's license (see here for details). Make sure you look at the specific community's copyright policy. Currently, you are viewing the copyright policy for the Default wiki. The Creative Commons License for this community is shown below. See the medshelf.org homepage for a list of all communities.

\section{Creative Commons License}

\section{Under this license you are free:}

- $\quad$ to copy, distribute, display, and perform the work

- to make derivative works

- to make commercial use of the work

\section{Under the following conditions:}

- Attribution. You must attribute the work in the manner specified by the author or licensor. At MedShelf.org you must attribute the work to the specific community wiki project that it is taken from. The easiest way to do this is to link to the 
specific community's wiki Main Page (e.g., http://communityname.medshelf.org), where communityname is replaced by the actual community name. You are currently viewing the policy for the Default wiki.

- For any reuse or distribution, you must make clear to others the license terms of this work.

- Any of these conditions can be waived if you get permission from the copyright holder.

Your fair use and other rights are in no way affected by the above.

This is a human-readable summary of the Legal Code (the full license).

$\underline{\text { Creative Commons Disclaimer }}$

\section{Project:Disclaimer}

The MedShelf.org web site does not provide specific medical advice and does not endorse any medical or professional service obtained through information provided on this site or any links to or from this site. MedShelf.org is for informational purposes only and is not authored or reviewed by medical professionals. Medical information changes rapidly and therefore, some information on MedShelf.org may be out of date, and/or contain inaccuracies or typographical errors. Readers are encouraged to confirm the information contained herein with other sources including medical professionals.

Use of the MedShelf.org web site does not replace medical consultation with a qualified health or medical professional to meet the health and medical needs of you or others. If you have or suspect you have an urgent medical problem, promptly contact your professional healthcare provider.

Any application of the information obtained from MedShelf.org or sites linking to or from it is at the reader's discretion. The University of Michigan, researchers working on the MedShelf.org project, and members of the communities that use MedShelf.org are not liable for any direct, indirect, consequential, special, exemplary, or other damages resulting from use of this website or any web sites that link to or from it.

\section{Project:Privacy policy}

This page describes the privacy policy for all MedShelf.org community wikis. More information on the research project associated with MedShelf.org can be found here.

\section{Introduction}

MedShelf.org is created and maintained by a group of researchers at the University of Michigan's School of Information. However, content is provided by members of 
participating online medical support communities and the general public. The site has no commercial interests and will not provide any personal information to third parties. All activity on the site is stored and used for research purposes. This is done according to well-established ethical guidelines developed with the University of Michigan's Institutional Review Board. Throughout, the rights and privacy of individuals will be respected. The remainder of this page describes our specific practices.

\section{Data collection}

\section{Reading content}

You can browse MedShelf.org without telling us who you are or revealing any personal information about yourself. As part of our Research Project, we will identify which pages were accessed most frequently, using IP addresses as a measure of reading participation. No IP addresses will be made public or shared with any third parties and data will be presented in aggregate in any publications.

\section{Contributing content}

It is possible on some MedShelf.org community wiki's to contribute to the wiki without logging in to the system. In such cases, an IP address is stored and linked to all contributions, which are accessible on a user contribution page and in the Recent Changes page.

Contributors are encouraged to register with the site by creating a username and password, and optionally providing a full name and email address. Registering requires that "cookies" (small files on your hard drive) are enabled. They are used to help identify you and maintain your logged-in status. When registered users contribute to the wiki after they are logged in, their contributions are linked to their username. It is easy to view all contributions from a single user by visiting their user contribution page or recent contributions on the Recent Changes page. Usernames need not be real names. If they do reflect real names, readers of the wiki will be able to track your activity on the site. Even when a username does not reflect a real name, if personal information is provided by you somewhere on the wiki (e.g., on your user page - such as User:DerekHansen), readers of the site will be able to associate the personal information you have provided with your username.

Providing an email address allows the system to email you when pages that you are watching are modified. The email address is not shown publicly anywhere on the site. We will not share the email addresses with any third parties or use them for advertising. We may, however, use them to contact contributors to ask if they would be willing to be interviewed or answer survey questions as part of our research project. 
Users who have provided an email address may also directly contact (and be contacted by) other registered users. This is done by using the "Email this user" link on a person's user page. If someone contacts you by using the link, they are not provided with your email address. However, if you send an email to another person using the link then they will be provided with your email address in the "From:" portion of the email message. This allows them to reply to you directly. So, please do not send email to other users unless you are ok with them knowing your email address.

\section{Watchlists}

Registered users can create watchlists that help them keep track of pages they are interested in. The watchlist of one user is not accessible by other users. However, we may use the watchlists for research purposes.

\section{Community Email List and Forum content}

In addition to analyzing the wiki content, the research project affiliated with MedShelf.org will also be looking at email or forum messages sent to the some of the associated medical support groups. We may also interview individuals and send occasional community surveys. This will only be done after introducing ourselves to the communities via their community email list (or forum) and with the approval of the list owners. When possible, we will associate wiki contributors to email list (or forum) members. This will be done by comparing email addresses and real names (if provided when logging into MedShelf.org). We may also ask individuals to voluntarily provide us

with their MedShelf.org usernames and email list addresses so that we can associate them together.

\section{Personal communication}

As part of our research project, we will be contacting participants of MedShelf.org and the communities that are affiliated with it. We will do so using the email addresses provided by registered users or addresses from the community email lists (or forums). We may also invite participants to contact us via email by posting a message on the wiki itself.

When contacting others we will always indicate who we are and what we are doing. We will respect the wishes of anyone who does not wish to interact with us. We would need to get parental consent for any direct contact (e.g., private email) with anyone under 18 years old. When we initiate contact, we will make every effort to contact only people who are 18 or older. If you are under 18 and we mistakenly contact you, please ignore our email. 


\section{Data retention and use}

We will save the data we have collected until we have completed all our analyses. The data will be used in academic conference presentations and published journal articles.

The data that we gather will remain confidential to the extent allowable under local, state, and federal law. We may quote what individuals have said or written. When this is done, we will not refer to the author by name or username. When possible, we will seek permission to include direct quotes from the authors of wiki entries and email/forum messages. We will not include any quotes from authors who indicate that they do not want us to. In all cases, we will not publish any personally identifiable information. Likewise, all numerical data presented in publications will be aggregated so that individuals cannot be identified.

\section{Contact information}

If you have any questions or concerns about our project, please contact Derek Hansen at shakmatt at umich dot edu or by phone at 734.764.1008. You may also contact Paul Resnick at presnick at umich dot edu. If you have questions about your rights as a participant in the MedShelf.org research project, please contact:

Institutional Review Board

540 E. Liberty \#202

Ann Arbor, MI 48109

Phone: 734-936-0933

email: irbhsbs at umich dot edu.

\section{Help:Contents}

This page outlines the help pages that describe how to use the MediaWiki software used on MedShelf.org.

\section{Logging in and Preferences}

- Help:Logging in - describes how to register and log in to the wiki

- Help:Preferences - describes the Special:Preferences page

- $\quad$ Privacy policy - how data collected from MedShelf.org is used

\section{For beginners}

- $\quad$ Help:Wiki 101 - a simple example of how to edit a page (including screenshots)

- Wiki Guide - the best place to start from if you're new to this wiki

- Help:FAQ - answers the most common questions related to using the software 


\section{Navigation}

- Help:Searching - describes how to navigate through the site using the search box and other special pages (e.g., Special:Allpages)

- Help:Category - explains categories and how they can be used to organize pages

- Help:Namespace - explains namespaces and how to use them appropriately

\section{Editing}

\section{Wikitext and basic formating}

- Help:Wiki 101 - an example of how to make a simple edit

- Help:Editing - a basic editing introduction

- Help:Images and other uploaded files - how to upload and link to images and other files

- Help:Piped link - explains piped links and when to use them

- Help:Wikitext examples - a fairly comprehensive list of wikitext examples

\section{Edit page help}

- Help:Edit summary - explains the edit "summary box" and how to use it effectively

- Help:Minor edit - explains minor edits and when to flag an edit as minor

- Help:Show preview - explains how to view a preview before saving your edits

\section{Advanced editing}

- Help:Edit conflict - explains what to do when two people edit the same page at the same time

- Help:Link - advanced page describing internal and external links

- Help:HTML in wikitext

- Help:PubMed links - explains how to link to articles in the PubMed database using the PMID \#

- Help:Reverting a page to an earlier version - how to revert a page

- Help:Section - explains page sections, table of contents, and how to link to sections

- Help:Table - how to use and format tables

- Help:Template - describes what templates are and how they are typically used

\section{Page Management}

- Help:Organizing pages - how to link and organize wiki pages 
- Help:Protecting pages - how to protect pages (only for administrators)

- Help:Deleting pages - how to delete pages (only for administrators)

- Help:Redirect - explains redirects and how to use them effectively

- Help:Renaming a page - how to rename a page, also called "moving" a page

- Help:Starting a new page - how to create a new page

- Wiki Genre - describes what a Wiki Genre is and how they can help you consider how to organize and present the information on a page

\section{Tracking Changes}

- Help:Diff - describes "diffs" and how to interpret them (e.g., on page histories)

- Help:Image page - describes uploaded images and files and changes made to them

- Help:Page history - describes the page history pages

- Help:Recent changes - describes the Special:Recentchanges page

- Help:User contributions - describes user contribution pages (e.g., Special:Contributions/DerekHansen)

- Help:Watching pages - describes the Special:Watchlist page

\section{Administration}

- Help:Administrators - describes types of administrators

- Help:Administration - describes special administrator abilities

- Wiki Team and Wiki Helpers - lists this wiki's administrators and other volunteers

\section{See also}

\section{Off-site help pages}

- MediaWiki Help pages - not all MediaWiki features are exactly the same at MedShelf.org, but most of the information provided on MediaWiki's own help pages relates to this wiki as well.

\section{Other wiki pages of interest}

- Suggestion Box - for recommending improvements or problems with the wiki content or software

- Project:Getting Started and Project:Checklist - help the Wiki Team initially roll out the wiki

- About, Copyrights, Disclaimer, and Privacy policy - pages that describe the project and various policies related to it

\section{Special pages of interest}

- Special pages - a list of all automatically generated "special" pages 
- All pages - lists all pages in a given namespace

- Category pages - shows all existing and wanted categories

- File list - lists all images and files that have been uploaded along with some basic statistics

- List redirects - lists all redirect pages

- Logs - various logs that show various events (e.g., deleting pages, giving users new rights, protecting pages)

- Orphaned pages - lists pages that no other page links to

- User list - lists all registered users of the system and their status

- Wanted categories - lists categories that have been linked to, but are currently empty

- Wanted pages - lists pages that have been linked to, but are currently empty

\section{Wiki Team}

The Wiki Team is a small group of community members that helps oversee wiki activity and promotes the wiki within the community email list. You can contact them by sending an email to [insert Wiki Team email address]. You may also want to view the Wiki Helpers page that describes other helpers in addition to the Wiki Team.

\section{Current members include:}

- [Insert username and possibly real name. Insert any specific responsibilities next to the individual's name. For example, Clean Up Crew leader, Wiki Promotion leader, Wiki Digest Chief Editor.]

\section{Wiki Helpers}

There are many ways to help improve this wiki. You don't need any special privileges or authority to start making changes. However, we have identified some special roles for a few brave volunteers to fill. If you want to help fill any of these roles, let others know by adding your username to the appropriate list below.

\section{Clean Up Crew}

The Clean Up Crew helps by picking up litter (e.g., removing spam, deleting pages created on accident), making pages look consistent, fixing spelling and grammatical errors, updating broken links, adding internal links to relevant wiki pages, and improving navigation by renaming and reorganizing (e.g., categorizing pages). They regularly check the Recent changes page to monitor recent activity. They also maintain their personalized Watchlist by "watching" pages and getting email updates when they are modified.

The Clean Up Crew can always use more members that help with all, or some, of their activities. Note: If you would like to be able to delete pages, you will need special 
administrator rights. Please contact the Wiki Team who can provide you with those rights. Clean Up Crew members include:

- [insert username here]

\section{Promotion Crew}

The Promotion Crew helps promote the wiki to the support group email list and world at large. They reference relevant wiki pages in the email list conversation, announce new pages to the list, encourage others to contribute to the wiki, help answer newcomer questions about the wiki, and advertise the wiki to other communities. You may want to read through the best practices and suggestions on how to best promote the wiki.

Promotion Crew members include:

- [insert username here]

\section{Page Advocates}

Some members like to adopt a specific page (or pages) that they regularly monitor, improve, and advocate for. They often choose pages on topics related to their own expertise or interests. Page Advocates link to their page when related discussions come up in the email list. They may request comments from the community on the topic via email and then compile and synthesize them into their page. When they come across new information or Internet resources they add them to the page. They also generally Watch the pages they adopt so that they are notified by email when others change them.

Below is a list of Page Advocates and the pages that they keep track of.

- [insert username here] - [insert link to page(s) that you've adopted]

\section{Wiki Digest Editors}

The Wiki Digest is like a newsletter that is regularly sent to the community email list. It spotlights individual contributors, highlights new or exceptional content, suggests new page topics or pages in need of review, discusses policies made by the Wiki Team, provides tips for new wiki users, and asks for suggestions. See the Sample Wiki Digest for ideas on what it might end up looking like.

Below are the usernames of the Wiki Digest Editors:

- Chief Editor: [insert username]

- Assistant Editor: [insert username] 


\section{$\underline{\text { Wiki Team }}$}

The Wiki Team consists of a handful of individuals in charge of making policy related to the wiki, providing the initial wiki content, promoting the wiki, and making others administrators. If you are interested in joining the Wiki Team, send them an email and let them know (see the Wiki Team page for a list of members and contact information).

\section{Wiki Genre}

A Wiki Genre is a type of wiki page that acts as a template by suggesting the purpose of a page, as well as what it might look like. Contributors who create a new page are encouraged to think about what Wiki Genre (if any) would best suit their needs, although they do not have to follow one exactly. As with other genres (e.g., office memo, encyclopedia entry, tax form), Wiki Genres generally have a commonly understood purpose and similar elements of form (e.g., a header, references section, tables).

Below are pages explaining a few generic Wiki Genres. Feel free to add others. They include examples taken from elsewhere on the web. You may want to change or add new examples more specific to your community (e.g., by including wiki pages from this community wiki).

- Guide Genre - for walking someone through a collection of resources

- Links Genre - for organizing and describing information resources

- How To Genre - for describing "how to" do something

- Encyclopedia Genre - for providing background knowledge on a topic

- Definition Genre - for providing a definition

- Review Genre - for reviewing a product or service

- Comparison Genre - for comparing different options

- Essay Genre - for sharing personal stories or essays

\section{Project:Comparison Genre}

This page describes the Comparison Genre, a specific type of Wiki Genre.

\section{Purpose}

To compare different options. It can be especially useful for comparing hotly debated topics in a meaningful and neutral way.

\section{Common Elements}

This genre typically begins with a description of the different options and, when applicable, a description of why the topic is hotly debated. It then summarizes the key 
arguments (e.g., pros and cons) for or against the major options. This is often done in a table, as shown below. It should be presented in a neutral tone, even though individuals may have strong personal opinions about certain options. Additional resources from different viewpoints are often referenced at the end.

\section{Examples}

- Disease Modifying Drugs for MS - a brief description of the options and a simple table comparing a few features of the various drugs (including links to their websites).

- $\quad$ Symptom comparison chart - only includes a comparison table.

- $\quad$ Treatment Options for Bipolar Disorder - does not include a comparison table, but still fits well within the scope of a Comparison Genre.

\section{Common Wikitext}

Here is an example of a table that can be used for this genre. This table compares three competing options, but it can be modified to account for fewer or more options.

\begin{tabular}{|c|c|c|c|}
\hline & Treatment 1 & Treatment 2 & Treatment 3 \\
\hline $\begin{array}{l}\text { Brief } \\
\text { description }\end{array}$ & $\begin{array}{l}\text { An outpatient surgery } \\
\text { where... }\end{array}$ & $\begin{array}{l}\text { A prescription drug } \\
\text { treatment including... }\end{array}$ & $\begin{array}{l}\text { An over-the-counter } \\
\text { drug treatment } \\
\text { including... }\end{array}$ \\
\hline $\begin{array}{l}\text { Likelihood of } \\
\text { success }\end{array}$ & $\begin{array}{l}\text { very high. Symptoms } \\
\text { not likely to return... }\end{array}$ & $\begin{array}{l}\text { very high. Symptoms } \\
\text { are reduced while on } \\
\text { medication... }\end{array}$ & $\begin{array}{l}\text { marginal improvement } \\
\text { at best }\end{array}$ \\
\hline Cost/Insurance & $\begin{array}{l}\text { Cost is generally high, } \\
\text { but is covered by most } \\
\text { insurance plans }\end{array}$ & $\begin{array}{l}\text { Cost is not as high as } \\
\text { Treatment } 1 \text {, but } \\
\text { copays can be high } \\
\text { for insurance plans }\end{array}$ & $\begin{array}{l}\text { Cost is minimal and is } \\
\text { rarely covered by an } \\
\text { insurance plan }\end{array}$ \\
\hline Side Effects & $\begin{array}{l}\text { Scarring and typical } \\
\text { short-term side effects } \\
\text { associated with general } \\
\text { anesthesia. See [link] for } \\
\text { more details. }\end{array}$ & $\begin{array}{l}\text { Most common ones } \\
\text { include nausea, .... } \\
\text { See [link] for more } \\
\text { details. }\end{array}$ & $\begin{array}{l}\text { Generally no side } \\
\text { effects, although some } \\
\text { people report... See } \\
\text { [link] for more details. }\end{array}$ \\
\hline
\end{tabular}




\section{Project:Definition Genre}

This page describes the Definition Genre, a specific type of Wiki Genre.

\section{Purpose}

To describe what a term means and how it is used by the community.

\section{Common Elements}

Definitions are brief descriptions of the meaning of a specific word, phrase, or acronym. They are written in the third person. They may describe what special significance the term has to members of the community. They may also include examples of how the word is used by the community and how to pronounce it.

On a Wiki it is recommended that each definition be created as a separate page, although they can be listed in alphabetical order on a master page. This allows people to link to each word's definition from within another page, rather than linking to a large list of all definitions.

\section{Examples}

- $\quad$ Medterms dictionary - one popular example of an online medical dictionary intended for medical practitioners

- $\quad$ GIST Support glossary - includes definitions of terms related to GIST. Unlike some medical dictionaries intended for doctors, these definitions often include comments for GIST patients.

\section{Project:Encyclopedia Genre}

This page describes the Encyclopedia Genre, a specific type of Wiki Genre.

\section{Purpose}

Provide a brief, but comprehensive write-up of a specific topic.

\section{Common Elements}

Encyclopedia entries are written in an objective, third person voice. They include relevant facts meant to provide an overview of the topic. They also include references to more 
detailed write-ups and links to articles on related topics (usually at the bottom of the page in a separate section).

\section{Examples}

- Wikipedia - includes many examples of this type of genre in a wiki.

- MedlinePlus Encyclopedia - includes example of this type of genre in the medical arena.

\section{Project:Essay Genre}

This page describes the Essay Genre, a specific type of Wiki Genre.

\section{Purpose}

To provide personal experiences and reflections on a particular topic.

\section{Common Elements}

Essays and personal stories are written in the first person. They generally include not only what happened, but what the author was thinking and feeling. This genre may be organized into sections, but is made up of typical prose. They are generally signed by the author, although in this public setting it may be more appropriate to sign with a pseudonym or false name. On a wiki, people other than the original author can leave their comments about the essay in a separate comments section at the bottom of the page.

\section{Examples}

- Personal Stories of mothers who have dealt with postpartum depression.

- Essays from cancer sufferers found at the GIST Support community site.

\section{Project:Guide Genre}

This page describes the Guide Genre, a specific type of Wiki Genre.

\section{Purpose}

To introduce a topic and set of resources to an individual who is unfamiliar with it. 


\section{Common Elements}

Guides are generally written in a collegial and empathetic tone from the perspective of someone who has "been there" before. It is meant to present material in an organized way, starting with the basics and moving on to more advanced topics. Guides can be long. They generally include a mixture of background information, resources (with descriptions about why they are good or not complete), suggestions, and personal experiences.

\section{Examples}

- $\quad$ Your Guide to the Breast Cancer Pathology Report - this guide is more detailed and lengthy than a typical wiki guide page would be, but the tone and content is reflective of the Guide Genre.

- $\quad$ A Guide for Students with Depressed Friends - this guide is around the same size as a wiki guide might be and the tone and content are similar, however it doesn't link to other resources as much as it could.

\section{Project:How To Genre}

This page describes the "How To" Genre, a specific type of Wiki Genre.

\section{Purpose}

To describe how to do something, not necessarily what it is or why it works.

\section{Common Elements}

How To pages begin with a short description of the goal and perhaps a few reasons why people need to know how to do it (e.g., "This page describes how to understand a typical blood test lab report. People diagnosed with... generally use blood tests to measure..."). It then describes (in order) the steps necessary to accomplish the goal. When there is more than one way to accomplish the goal, each method is described in a separate section. They often link to other resources that are more comprehensive, but they are meant to stand on their own.

\section{Examples}

- $\quad$ How to Write Your Own Health Care Proxy article - taken from http://www.eHow.com. This page and other health related "how to" articles may be more rigid in their format than necessary (e.g., listing everything as a numbered step), but serve as decent examples of what might fit in this genre. 
- How to Understand Your BMD Test Results article - a good example of this genre provided by the New York State Osteoporosis Prevention Education Program (NYSOPEP).

\section{Project:Links Genre}

This page describes the Links Genre, a specific type of Wiki Genre.

\section{Purpose}

To help people easily find appropriate and high quality resources.

\section{Common Elements}

This genre consists of hyperlinks to resources with brief descriptions of each resource. It is similar to an annotated bibliography. Resources may include pages within this wiki, elsewhere on the Internet, or even in print. Resources are often provided in alphabetical order or organized into appropriate sub-groups to help people find them.

\section{Examples}

- Gastroenterology resources - a fairly comprehensive list of links with short descriptions. This is probably a bit longer than most Wiki Links pages would be, but is a good example of the genre.

- IPL alternative medicine resources - a great example of brief annotations that provide insights about the source and content of each resource.

\section{Common Wikitext}

Here is an example of a table that can be used for this genre, including a few different types of links. 


\begin{tabular}{|c|c|}
\hline Resource & Description \\
\hline Wiki Genre & $\begin{array}{l}\text { This is an internal link to a wiki page. The page includes a description } \\
\text { of several Wiki Genres and links to a few different examples such as } \\
\text { the Link Genre, Guide Genre, and Debate Genre. }\end{array}$ \\
\hline Medline Plus & $\begin{array}{l}\text { This is an example of an external link to a website. Medline Plus is a } \\
\text { collection of articles on medical topics written by medical } \\
\text { professionals for patients. It is sponsored by the National Library of } \\
\text { Medicine. }\end{array}$ \\
\hline$\underline{\underline{\text { ISBN }}} \underline{\underline{1930683006}}$ & $\begin{array}{l}\text { The Self-Help Group Sourcebook - This is a book that lists all kinds } \\
\text { of self-help groups, as well as summarizes research on how to } \\
\text { effectively run one. }\end{array}$ \\
\hline PMID 14713653 & $\begin{array}{l}\text { Adolescents searching for health information on the Internet - This } \\
\text { article discusses some common search strategies and challenges that } \\
\text { youth face when searching for health information online.) }\end{array}$ \\
\hline
\end{tabular}

\section{Project:Review Genre}

This page describes the Review Genre, a specific type of Wiki Genre.

\section{Purpose}

To provide information that will help others make an informed decision about a product or service.

\section{Common Elements}

Reviews are written from the perspective of someone who has used the product or service. Good reviews begin by objectively describing what the product or service is for and how it works and may even include a photograph of the product (learn how to upload images on this wiki). Authors then describe what they liked and didn't like and why. In a wiki, each reviewer may create a subsection with their own review and sign their comments (with their username or some other pseudonym). Links to other reviews and resources about the product or service are generally included. Reviews on this wiki would emphasize how the product or service relates to the medical condition and be targeted at other community members rather than the general public.

It may make sense to create a page that links to several specific product review pages. For example, a Project:Guide Genre page titled Purchasing a Wheelchair could be created that would give a brief overview on what to look for when purchasing a wheelchair and where to buy them from. It could then link to specific wiki review pages created for each major wheelchair product. 


\section{Examples}

- Amazon - includes a single page that has a photo of the product, description of the major features, and individuals' product reviews.

- $\quad$ Pricegrabber - includes similar information as Amazon.com but in a different format.

\section{Project:Sample Intro Email}

This page includes content that can be used to introduce the wiki to your community. It is written for email list communities, although forum-based communities can modify it for their purposes. The message should probably be sent from an admin of the email list, or at least explain that they are supportive of the project. If the person heading up the wiki project is different than the person sending this message, the email should recommend contacting the wiki project leader.

Below is a sample email message that will need to be customized to fit the needs of your community. It is written as if sent to the fictitious ABC Support Group. It also assumes that there is already a static ABC Support Group website. Words that will need to be changed are in italics.

\section{Return to Simple Start or Getting Started or Checklist}

\section{Email Content}

ABC Support Group members,

We have begun a new community project that we want to introduce. We are excited about the new possibilities that it will offer our community and hope that you will share our enthusiasm. We are particularly interested to hear from those of you who would like to help out with the project, as well as any of you who have concerns.

The project is to create a website that will act as our community's long-term memory. It will also allow us to share our experiences and expertise more widely. Unlike our current website, this one will be editable by all of you, and we hope it will really be a community effort. We are still thinking about what content it will include, and would love your input. So far we expect to have pages about coping with $A B C$ disease, personal stories, materials for newly diagnosed patients, links to web resources, traveling with $A B C$, parenting $A B C$ children, and a $A B C$ glossary and acronym dictionary. Are there other topics that are not well covered by existing websites? Are there topics that are shared within our email list that we want the world to know about? Are there resources that we want to create for our own community? 
Before making the site publicly accessible, we want to seed it with content that will make it useful from the start. If any of you are interested in helping create the initial content, please let us know. Once the initial content is created all of you will be invited to read and contribute to it.

The project is also part of a research study performed by researchers at the University of Michigan's School of Information. The goal of the study is to learn how online support communities like ours can benefit from these community-created websites. The research team is providing the technology (a customized wiki) and suggestions on how to best use it. However, we are free to do with it what we want. We have spoken with Derek Hansen, who is heading up the project and feel comfortable with the ethical procedures they are using and the long-term sustainability of the project. You can visit their website that describes the project and their ethical procedures at http://wwwpersonal.si.umich.edu/ shakmatt/support/index.html

You can also contact Derek Hansen directly at shakmatt@umich.edu if you have any questions or concerns. He will send out a separate email introducing himself to our community soon.

We are excited at the potential of this project to help our community and others that suffer from $A B C$ Disease. We will send out a link to the website once we have put in some initial content. Let us know if you're interested in helping out or if you have any concerns about the project.

Signature

\section{Project:Sample Main Page}

Below is a sample Main Page to give you an idea of how you might want to structure the content. The Main Page is the first page that people will see when coming to the wiki. We recommend that it serve as an organized index to the content that is in the wiki. Your community wiki will include different pages and sections than this sample because of its unique focus. However, you may get some ideas from this sample page that you can then tailor to your specific needs when you edit the actual Main Page. But remember to start simple. You may only want to have a handful of pages to begin with and add more over time.

The Sample Main Page is for the imaginary ABC Support Group. Some of the links in this page are to real pages with content. When no real page exists (since this is only a sample), we have put the imaginary page name in bold. There are also comments in green that would not be included in the actual Main Page. Most of the comments identify the type of page (i.e., Wiki Genre that our hypothetical pages would be).

Welcome to the ABC Support Wiki! This wiki serves as the community's long-term memory and public outlet to the world. Please read our About page and Disclaimer and understand that the information found in this Wiki is not provided by medical experts. 
We hope that you find the content useful and help improve it by making your own edits. After all, it is a community project run by individuals like you!

\section{ABC Support Community}

- Please visit our ABC Support website note: this would of course be a real link to a publicly available community webpage if you have one.

- ABC Support Email List - Information on how to join and unsubscribe from the ABC Support email list, as well as Posting Guidelines that should be read before posting to the list.

- $\quad$ List Archives - Only accessible to subscribed members of the email list.

- ABC Support Members - Meet some of our ABC Support members. note: this page could include links to personal webpages, links to wiki user pages, and/or personal essays or stories (see Essay Genre)

\section{Using the Wiki}

- $\quad$ First time wiki user? See our Wiki Guide

- Feeling experimental? Learn how to edit and then try editing the Sandbox.

- Share your recommendations on how to improve the wiki at the Suggestion Box or volunteer to become one of our Wiki Helpers.

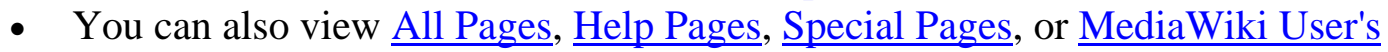
Guide.

\section{About ABC}

- ABC Overview note: probably an Encyclopedia Genre

- ABC Resources note: probably a Links Genre

- Facts and Figures - some basic statistics on ABC

- Definitions note: probably a list of links to specific wiki Definition Genre pages. Each definition is generally best kept separate since they will be linked to from other wiki pages.

- Acronyms note: similar layout as Definitions page

\section{For New ABC Patients \& Friends}

- ABC Orientation note: probably a Guide Genre

- $\quad$ Test Results - what you need to know to understand your common ABC related test results note: probably a How To Genre

- Breaking the News - ideas on how to let your friends and family know you have ABC 


\section{Treatment Options}

- Treatment Overview - introduces the various treatment options note: each option would likely have it's own Encyclopedia Genre wiki page and may include descriptions of how to prepare for the treatment as well as an overview of the treatment itself

- Treatment Chart note: a Comparison Genre

- Clinical Trials note: probably a Links Genre

\section{Ongoing Learning}

This section includes pages that help members learn more about ABC.

- ABC Research - an annotated list of websites discussing current research note: probably a Links Genre

- ABC Resources note: probably a Links Genre

- $\quad$ ABC Books note: probably a Links Genre that lists books and links to reviews of the books, each one a Review Genre

- Related Organizations note: probably a Links Genre

\section{Managing $A B C$}

This section includes pages on coping and dealing with the every-day life situations that arise from $\mathrm{ABC}$.

\section{Coping with $\mathrm{ABC}$}

- Stories of Strength note: probably an Essay Genre

- Advice for Parents, Advice for Friends, Advice for Spouses

- Living in a Hospital - how to make your hospital stay as comfortable as possible

- ABC Essays note: probably an Essay Genre

- Coping Strategies

- ABC Humor

\section{Working with Medical Professionals}

- Questions for Your Doctor

- Finding Good Care note: probably a How To Genre or a Guide Genre.

- Educating Your Doctor - what every doctor should know about ABC and how to encourage them to be more informed 


\section{Medical Equipment}

- ABC Product Guide - an introduction to what ABC products and services are available note: probably a Guide Genre, but may link to Comparison Genre pages that compare certain product types

- Product Reviews - find out what community members think of ABC products, services, and vendors. note: probably a list of links to specific Review Genre wiki pages

- Finding Products - links to ABC product vendors, as well as tips on finding cheap and used ABC products

- Exchanging Products - our guidelines for using the ABC Support email list for product exchanges

\section{Insurance}

- Insurance Stories - our member's experiences in dealing with their insurance companies note: probably an Essay Genre

- Covered Services - a description of what ABC related services should be covered and which are not generally covered

- Appealing a Claim - suggestions on appealing claims for common ABC-related treatments note: probably a

\section{Community Projects}

This section includes pages related to current or proposed community projects.

- Recipes - a collection of recipes ideal for people with ABC note: this page would probably link to individual wiki pages, each representing a recipe (a type of $\underline{\text { How }}$ To Genre)

- Fund Raising Activities - a description of current fund raising activities by ABC Support members

- ABC Support Mission Statement - We are currently reviewing our Mission Statement. Please take a moment to add your thoughts.

\section{Project:Sample Roll Out Email}

This page includes text that can be customized and used once you have completed seeding the wiki with content and are ready to introduce it to the entire community. The message should probably be sent by the Wiki Team Leader. It is written for email list communities, although forum-based communities can modify it for their purposes.

The sample message is written as if sent to the fictitious ABC Support Group. Comments in italics will need to be updated. You will likely want to customize it a bit more as well, 
perhaps pointing out some of the major sections of wiki content and suggesting areas that could use some immediate attention by other members.

Return to Simple Start or Getting Started or Checklist

\section{Email Content}

[ABC Support Group] members,

We have finished creating the initial content for our new community website (i.e., wiki)! Come check it out at [http://abcsupport.medshelf.org]. Thanks to all of you who gave us ideas on what should be included. We hope that you will find it useful and that you will help improve it by creating your own pages and editing the existing pages. After all, it is a community project and the more involvement we have, the better it will be!

To help people keep up on new content that is added to the wiki and learn how to use it effectively, we will send out a Wiki Digest email on a regular basis. Also, if you have any questions about the wiki, you can send a message to the list or to one of the Wiki Team members.

Here are a few ideas on how you can get involved. - Read the Wiki Guide [1], which will explain how to use the wiki and make your own contributions. Then browse through the existing pages and make your own additions and edits. - Use the wiki content in our ongoing email list discussion: Link to specific wiki pages to help you ask or answer questions; discuss pages that should be improved or updated; announce new wiki pages that you have created; suggest email threads that should be summarized and added to the wiki. - Consider becoming one of the Wiki Helpers [2] by adopting a specific wiki page to keep updated, helping promote the wiki, or joining the Clean Up Crew.

We are excited about this new community project and look forward to seeing how you will all shape it into a more useful resource for our community and for those who suffer from $A B C$ disease.

Sincerely, The Wiki Team

[insert names of Wiki Team members]

[1] http://abcsupport.medshelf.org/Wiki_Guide

[2] http://abcsupport.medshelf.org/Wiki_Helpers

\section{Project:Sample Wiki Digest}

The Wiki Digest is like a newsletter about the wiki that is sent out regularly by the Chief Wiki Digest Editor to the community email list or forum. For active communities this may be once a week. For less active communities this may be every other week or once a 
month. Below is a sample Wiki Digest to give the editors ideas on what types of content may be included. It is written for the fictitious ABC Support Group with fictitious participant names and wiki pages. The formatting has been removed so it looks more like a plain text email message.

\section{Sample Wiki Digest Content}

\section{Subject: Wiki Digest \#1}

\section{Body:}

ABC Community members,

Thanks to all of you who have continued to help improve our community wiki! If you haven't yet visited the site, there is already a number of great resources on the wiki found at http://abcsupport.medshelf.org that you'll want to check out.

\section{WIKI CONTRIBUTOR RECOGNITION}

Derek is our wiki contributor this month. You can see all of his contributions at [1]. We especially liked his additions to the *Questions_for_Your_Doctor* page [2]. Derek has not only added a great deal of content to existing pages, he has also referenced specific wiki pages in our email list discussion to help others find relevant information. Great work Derek!

\section{WHAT'S NEW}

Several notable new pages have been created this month that you'll want to check out and add to including...

1. *Acronyms* - a page that lists common ABC disease related acronyms and their explanations.

2. *ABC_Orientation* - a guide for patients newly diagnosed with ABC disease. This page has a great start, but there are likely other web resources that should be included so if you know of good ones make sure and add them in.

3. *Stories_of_Strength* - 2 new stories were added to the Stories of Strength section, so there are now a total of 6. One of the new pages (Dans Story [3]) is our first story told from the perspective of a spouse. As always, make sure if you post a story that you don't use your real name unless you are comfortable with everyone (including non-community members) knowing that it is about you. You may also want to make the edits as an anonymous user so your username doesn't show up.

\section{PAGE OF THE MONTH}


We want to draw your attention to an exceptional page, thanks in part to the hard work of Derek. The page, *Questions_for_Your_Doctor* [2] gives some great ideas on what you should ask your doctor about ABC disease. Although we discuss this topic frequently in the email list, until now there has not been a resource that compiles all of the ideas in a single place. Thanks to all of you who have contributed to the page in the past, and remember, there is always room for improvement.

\section{HELP REQUESTED}

The Main Page has grown a bit too long lately. In particular, the section on "Coping with $A B C "$ is a bit chaotic and overgrown. Some help in organizing the content in that section into a more coherent whole would be great. It may require creating some new summary pages that can be linked to from the Main Page.

Several people have mentioned the need for a page discussing all of the organizations that might be of interest to those of us with ABC disease. I have created a new page

*Related_Organizations* [4] that can serve as a pointer to organizations of interest with a brief description of each of them. Unfortunately, I don't really know too many of them and would love to get all of your input. You can either email me with suggestions or (even better) edit the page yourself.

We have a page called *Living_in_a_Hospital* [5] that discusses a topic that is often raised on the email list. There are some good ideas on the page, but many of the best ideas that have been raised in the email list are not on the page. It would be great if some brave souls would look in the email archive (and their own experience) and summarize some of the best ideas on the page.

\section{WIKI POLICIES}

The Wiki Team wants to remind everyone that it is appropriate to discuss wiki pages and edits on the email list at any time. Even if not every member is interested in the specific comments being made, it is a good way to keep up-to-date on what is happening on the Wiki and often leads to fruitful discussions in the email list. We do ask that members include the word "wiki" in the subject line of the email if the question is solely about the wiki.

\section{WIKI TIP}

A page is created for each person that has logged in (i.e., has a username). The page does not have any content on it to begin with. Some of you may want to add a brief description of yourself on your personal page. As always, make sure you do not include any details that you do not want shared with the whole world. You can see a list of all Users at the *Special:Listusers* page [6]. Just click on your username and it will take you to a page that you can edit. From then on, when your username shows up on other pages (e.g., *Special:Recentchanges*) it will link to your user page. 


\section{FINAL NOTE}

If anyone has ideas on content for the next Wiki Digest, feel free to email any of the Wiki Digest Editors. Many of our ideas come from members.

EDITORIAL BOARD

Chief Editor - Joe Smith

Assistant Editor - Sarah Jones

\section{REFERENCES}

[1] http://abcsupport.medshelf.org/User:DerekHansen

[2] http://abcsupport.medshelf.org/Questions_for_Your_Doctor

[3] http://abcsupport.medshelf.org/Dans_Story

[4] http://abcsupport.medshelf.org/Related_Organizations

[5] http://abcsupport.medshelf.org/Living_in_a_Hospital

[6] http://abcsupport.medshelf.org/Special:Listusers 


\section{APPENDIX 4 \\ COMMUNITY RECRUITMENT EMAILS}

\section{First Version of Community Recruitment Email}

Katie,

My name is Derek Hansen. I am a Ph.D. student at the University of Michigan's School of Information. Professor Paul Resnick and I are working to improve online support groups like yours. We do this by helping them create community repositories (i.e., like a multi-authored FAQ). We are looking for online support groups that would like to add a community repository to their current email-based conversation. If you think your community would benefit from such a repository, please let us know by replying to this email. We can then explain more about our current project and how we can work together.

We've included more details below and at the following site [http://www-personal.si.umich.edu/ shakmatt/support/index.html] for your review. Thank you for your time. Please don't hesitate to email (shakmatt@umich.edu) or call me (home \#: 734-764-1008) if you have any further questions.

Regards,

Derek Hansen

Doctoral Candidate

School of Information

University of Michigan

Purpose of Study 
Online support groups help people share information and emotional support across time and space. Most of these groups use email lists or online forums to communicate. More recently, new technologies (e.g., Wikis) have allowed large groups to coauthor shared documents. Some groups have used these new technologies to create "community repositories." These repositories include frequent questions and answers, biography pages, group policies, and other content designed to help the group. We hope to better understand the potential benefits of such repositories. We also hope to learn how to best introduce them to existing email-based groups.

Research Procedures

Our research approach is based on working with existing online support groups. We do this by providing the technology (i.e., a Wiki) needed for coauthoring web pages. We also provide groups with "training" materials. These include best practices for creating a useful community repository. The support groups are then free to use these resources to meet their unique needs, tailoring them as desired. The repository is a separate website. As such, support groups need not modify their current email list software.

We also hope to identify general lessons that will help similar groups. To do this, we interview willing members, offer occasional group surveys, and analyze email messages and repository content. The rights and privacy of individual members will be respected throughout. We will comply with well-established ethical guidelines developed with the University of Michigan's Institutional Review Board. These are outlined at [http://www-personal.si.umich.edu/ shakmatt/support/index.html]. Support groups and their members can stop participating at any time and are not required to participate.

We will initially host the repository for a year. At least three months prior to the end of the year we will discuss two possibilities. The first is to extending the hosting for a longer period of time. The second is to assist with the transfer of the repository to a different host of your choosing. In the second case, we will provide the data in a format 
that can be transferred. The repository will also be built using popular and freely available Wiki software. This means that groups will be able to exactly replicate the technology.

Repository Example

We are currently looking for medical support groups to work with. Because the technology is fairly new, we do not have an example of a medical support group's repository. However, we do have an example of a technical support group's repository. The group is for people that use Cascading Style Sheets (CSS) in website design and is called CSS-Discuss. The community repository or Wiki is found at http://cssdiscuss.incutio.com, and the public email archive is found at http://archivist.incutio.com/viewlist/css-discuss.

Although we have studied the CSS-Discuss group, we had nothing to do with the creation of their repository or public archives. The repositories that we will help your community create will not use the exact same software or contain the same types of information. However, the relationship between the email list and repository will be similar. In fact, the CSS-Discuss community may give you some ideas of how a repository could help your community. The CSS-Discuss repository is filled with pages that grew out of frequently asked questions or hot topics discussed on the email list (e.g., http://css-discuss.incutio.com/?page=ThreeColumnLayouts;

http://css-discuss.incutio.com/?page=ConstantsInCSS). It also includes member pages and definition pages. In addition, the email list often uses the repository pages to help answer questions (e.g., http://archivist.incutio.com/viewlist/css-discuss/71757) and coordinate efforts (e.g., http://archivist.incutio.com/viewlist/css-discuss/69786). Hopefully these examples will convey, albeit imperfectly, the general idea of what we envision helping your community create.

Contact Information 
Like you, we recognize the tremendous benefits that members of online support groups provide one another. We hope you will sincerely consider working with us to help your community and others like it reach their potential. Let us know if you are interested!

If you have any questions or concerns, please don't hesitate to email me (shakmatt@umich.edu) or call me at my home number (734-764-1008). Also, if you have any questions or concerns about your rights as a participant in research, please contact: Institutional Review Board

540 E. Liberty \#202

Ann Arbor, MI 48109

Phone: 734-936-0933

email: irbhsbs@umich.edu.

We look forward to getting to know you.

Sincerely,

Derek Hansen

Graduate Student

University of Michigan

School of Information

734-764-1008

Paul Resnick

Professor

University of Michigan

School of Information

\section{Second Version of Community Recruitment Email}

[community name] list owner(s), 
My name is Derek Hansen. I am a PhD student at the University of Michigan's School of Information. Professor Paul Resnick and I study technologies that help online support groups like yours. In our current study, we give support groups access to their own wiki - a special website that anyone can edit. We also give suggestions on how the website can be used and how to make it work well with an existing email list (such as the one you use at Yahoo). You can get an idea of how the site will work by visiting our sample wiki site at: http://default.medshelf.org

If you think your group would benefit from such a website, please let us know by replying to this email. We are currently recruiting groups that will start using the wiki in March 2007, so please contact us as soon as possible so we can plan accordingly.

We've included more details about the study at the following site:

[http://www-personal.si.umich.edu/ shakmatt/support/index.html]

We hope you will sincerely consider working with us to help your community and others like it reach their potential. If you have any questions about the study don't hesitate to email me at shakmatt@umich.edu or call me (home \#: 734-764-1008).

Regards,

Derek Hansen

Doctoral Candidate

School of Information

University of Michigan

Paul Resnick

Professor

School of Information

University of Michigan 


\section{BIBLIOGRAPHY}

Abdul-Rahman, A., \& Hailes, S. (2000). Supporting trust in virtual communities. Paper presented at the 33rd Annual Hawaii International Conference on System Sciences.

Ackerman, M. (2000). The Intellectual Challenge of CSCW: The Gap Between Social Requirements and Technical Feasibility. Human-Computer Interaction, 15(2-3), 179-204.

Ackerman, M., \& Halverson, C. (2000). Reexamining organizational memory. Communications of the ACM, 43(1), 58-64.

Ackerman, M., \& Malone, T. W. (1990). Answer Garden: a tool for growing organizational memory. Paper presented at the ACM SIGOIS and IEEE CS TCOA conference on Office information systems.

Ackerman, M., \& McDonald , D. (1996). Answer Garden 2: merging organizational memory with collaborative help Paper presented at the Proceedings of the 1996 ACM conference on CSCW.

Ackerman, M., \& Mcdonald, D. (2000). Collaborative Support for Informal Information in Collective Memory Systems. Information Systems Frontiers, 2(3-4), 333-347.

Ackerman, M., \& Palen, L. (1996). The Zephyr Help Instance: promoting ongoing activity in a CSCW system. Paper presented at the Proceedings of the SIGCHI conference on Human factors in computing systems: common ground.

Ackerman, M., Swenson, A., Cotterill, S., \& DeMaagd, C. (2003). I-DIAG: From community discussion to knowledge distillation. In Communities and technologies. Dordrecht: Kluwer Academic.

Anderson, J. R. (2004). Chapter 9: Expertise. In Cognitive Psychology and Its Implications (6th ed.). New York, NY: Worth Publishers.

Argote, L., \& Darr, E. (2001). Repositories of Knowledge in Franchise Organizations: Individual, Structural, and Technological. In G. Dosi, R. Nelson \& S. Winter (Eds.), The Nature and Dynamics of Organizational Capabilities (pp. 51-69): Oxford Scholarship Online Monographs.

Askehave, I., \& Swales, J. M. (2001). Genre Identification and Communicative Purpose: A Problem and a Possible Solution. Applied Linguistics, 22(2), 195-212.

Avison, D., Lau, F., Myers, M. D., \& Nielson, P. A. (1999). Action Research. Communications of the ACM, 42(1), 94-97.

Baskerville, R. (1999). Investigating Information Systems with Action Research. Communications of the AIS, 2(19).

Baskerville, R., \& Myers, M. D. (2004). Special Issue on Action Research in Information Systems: Making IS Research Relevant to Practice-Foreword. MIS Quarterly, 28(3), 329-335. 
Bates, M. (1989). The Design of Browsing and Berrypicking Techniques for the Online Search Interface. Online Review, 13(5), 407-424.

Bates, M. (1999). The invisible substrate of information science. Journal of the American Society of Information Science 50(12), 1043-1050.

Bazerman, C. (1988). Shaping Written Knowledge. Madison: University of Wisconsin Press.

Benders, J., \& van Veen, K. (2001). What's in a fashion? interpretative viability and management fashions. Organization, 8(1), 33.

Benkler, Y. (2002). Coase's penguin, or, Linux and The Nature of the Firm. Yale Law Journal, 112(3), 369.

Blazer, D. G. (1982). Social support and mortality in an elderly community population. American Journal of Epidemiology, 115, 684-694.

Bos, B., Çelik, T., Hickson, I., \& Lie, H. W. (2006). Cascading Style Sheets, level 2 revision 1 (working draft): W3C.

Braithwaite, D. O., Waldron, V. R., \& Finn, J. (1999). Communication of social support in computer-mediated groups for people with disabilities. Health communication, 11(2), 123-151.

Brewer, R. (2000). Improving problem-oriented mailing list archives with MCS. Paper presented at the Proceedings of the 22nd international conference on Software engineering.

Brin, S., \& Page, L. (1998). The anatomy of a large-scale hypertextual Web search engine. Paper presented at the Proceedings of the seventh international conference on World Wide Web 7, Brisbane, Australia.

Bryant, S. L., Forte, A. A., \& Bruckman, A. A. (2005). Becoming Wikipedian: transformation of participation in a collaborative online encyclopedia. Paper presented at the 2005 international ACM SIGGROUP conference on Supporting group work Sanibel Island, Florida, USA.

Burnett, G. (2000). Informational exchange in virtual communities: A typology. Information Research, 5(4).

Burnett, G., \& Buerkle, H. (2004). Information Exchange in Virtual Communities: A Comparative Study. Journal of Computer Mediated Communication, 9(2).

Burrows, R., Nettleton, S., Pleace, N., Loader, B., \& Muncer, S. (2000). Virtual Community Care? Social Policy and the Emergence of Computer Mediated Social Support. Information, Communication and Society, 3(1), 95-121(127).

Butler, B. (1999). The dynamics of cyberspace: Examining and modelling online social structure. Ph.D. Dissertation., Carnegie Mellon University, Pittsburgh, PA.

Butler, B. (2001). Membership size, communication activity, and sustainability: a resource-based model of online social structures. Information Systems Research, 12(4), 346-362.

Butler, B., Sproull, L., Kiesler, S., \& Kraut, R. (2002). Community effort in online groups: who does the work and why? In W. S. \& L. Atwater (Eds.), Leadership at a Distance. Mahwah, NJ: Erlbaum.

Button, G., \& Dourish, P. (1996). Technomethodology: paradoxes and possibilities. Paper presented at the SIGCHI conference on Human factors in computing systems: common ground, Vancouver, British Columbia, Canada. 
Chandler, D. (1997). An Introduction to Genre Theory. Retrieved July 23, 2007, from http://www.aber.ac.uk/media/Documents/intgenre/intgenre.html

Chang, S., \& Rice, R. (1993). Browsing: A multidemensional framework. Annual Review of Information Science and Technology, 28, 231-271.

Charon, R. (2001). Narrative Medicine. JAMA: the journal of the American Medical Association, 286, 1897-1902.

CHESS - Comprehensive Health Enhancement Support System. (n.d.). Retrieved July 23, 2007, from http://www.harthosp.org/cancer/PatientResources/CHESS/default.aspx

Clark, H. (1996). Using language. Cambridge, MA: Cambridge University Press.

Clark, H., \& Brennan, S. (1991). Grounding in communication. In L. Resnick, J. Levine \& S. Teasley (Eds.), Perspectives on Socially Shared Cognition (pp. 127-149). Washington, DC: APA.

Cohen, S., \& Wills, T. A. (1985). Stress, social support, and the buffer hypothesis. Psychological Bulletin, 98(2), 310-357.

Constant, D., Kiesler, S., \& Sproull, L. (1996). The kindness of strangers: On the usefulness of weak ties for technical advice. Organization Science, 7, 119-135.

Cosley, D., Frankowski, D., Kiesler, S., Terveen, L., \& Riedl, J. (2005). How oversight improves member-maintained communities. Paper presented at the SIGCHI conference on Human factors in computing systems.

Csikszentmihalyi, M. (1990). Flow: The Psychology of Optimal Experience. New York: Harper \& Row.

Csikszentmihalyi, M. (1996). Creativity: Flow and the Psychology of Discovery and Invention. New York: Harper Collins.

Css-d Public Archives. (n.d.). Retrieved July 23, 2007, from http://archivist.incutio.com/viewlist/css-discuss

Css-d wiki. (n.d.). Retrieved July 23, 2007, from http://css-discuss.incutio.com

Css-discuss.org. (2002). Retrieved July 23, 2007, from http://www.css-discuss.org

Culver, J., Gerr, F., \& Frumk, H. (1997). Medical Information on the Internet: A Study of an Electronic Bulletin Board. Journal of General Internal Medicine, 12(8), 466470.

Cunningham, W. (n.d.). WikiWikiWeb. Retrieved July 23, 2007, from http://c2.com/cgi/wiki?WikiWikiWeb

Cutrona, C. E., \& Russell, D. W. (1990). Type of social support and specific stress: Toward a theory of optimal matching. In B. R. Sarason, I. G. Sarason \& G. R. Pierce (Eds.), Social support: An interactional view (pp. 319-366). New York: Wiley.

Davison, K. E., \& Pennebaker, J. W. (1997). Virtual narratives: Illness representations in online support groups. In K. J. Petrie \& J. A. Weinman (Eds.), Perceptions of health and illness: Current research and applications (pp. 463-486). Singapore: Harwood.

Dery, M. (1993). Flame wars: the discourse of cyberculture. Durham, NC: Duke University Press.

DeSanctis, G., \& Poole, M. (1994). Capturing the complexity in advanced technology use: Adaptive structuration theory. Organization Science, 5(2), 121-147. 
Destailleur, L. (n.d.). Advanced Web Statistics 6.5. Retrieved July 23, 2007, from http://awstats.sourceforge.net

Dickerson, S. S., Flaig, D. M., \& Kennedy, M. C. (2000). Therapeutic connection: help seeking on the Internet for persons with implantable cardioverter defibrillators. Heart and Lung, 29, 248-255.

Dreyfus, H. L., \& Dreyfus, S. E. (2005). Peripheral Vision: Expertise in Real World Contexts. Organization Studies, 26(5), 779-792.

Dudley-Evans, T., \& St John, M. J. (1998). Developments in English for Specific Purposes: A Multi-Disciplinary Approach. New York: Cambridge University Press.

Durrance, J. (1995). Factors that influence reference success: What makes questioners willing to return? Reference Librarian, 49/50, 243-265.

Ebner, W., Leimeister, J. M., \& Krcmar, H. (2004). Trust in Virtual Healthcare Communities: Design and Implementation of Trust-Enabling Functionalities. Paper presented at the 37th Annual Hawaii International Conference on System Sciences.

Ehrlich, K., \& Cash, D. (1999). The Invisible World of Intermediaries: A Cautionary Tale. Computer Supported Cooperative Work, 8(1-2), 147-167.

Eisenhardt, K. M. (1989). Building Theories from Case Study Research. The Academy of Management Review, 14(4), 532-550.

Ellis, D., Oldridge, R., \& Vasconcelos, A. (2004). Community and Virtual Community. Annual Review of Information Science and Technology, 38(1), 145-186.

Emigh, W., \& Herring, S. C. (2005). Collaborative Authoring on the Web: A Genre Analysis of Online Encyclopedias. Paper presented at the 38th Annual Hawaii International Conference on System Sciences.

Erickson, T. (1997, 7-10 Jan 1997). Social interaction on the Net: virtual community as participatory genre. Paper presented at the 30th Hawaii International Conference on System Sciences, Wailea, HI, USA.

Erickson, T. (2000). Making sense of computer mediated communication (CMC): Conversations as genres, CMC systems as genre ecologies. Paper presented the 33rd Hawaii International Conference on System Sciences.

Esquivel, A., Meric-Bernstam, F., \& Bernstam, E. V. (2006). Accuracy and self correction of information received from an internet breast cancer list: content analysis. BMJ, 332, 939-942.

Eysenbach, G., Powell, J., Englesakis, M., Rizo, C., \& Stern, A. (2004). Health related virtual communities and electronic support groups: Systematic review of the effects of online peer to peer interactions. BMJ, 328(7449), 1166.

Feagin, J. R., Orum, A. M., \& Sjoberg, G. (Eds.). (1991). A Case for the Case Study. Chapel Hill, NC: The University of North Carolina Press.

Ferguson, T. (1996). Health Online: How to Find Health Information, Support Groups, and Self-Help Communities in Cyberspace. Reading, MA: Addison-Wesley.

Ferguson, T. (2007). e-Patients: How they can help us heal healthcare. Retrieved July 23, 2007, from http://www.e-patients.net/e-Patients_White_Paper.pdf.

Fernsler, J. I., \& Manchester, L. J. (1997). Evaluation of a computer-based cancer support network. Cancer Practice, 5(1), 46-51. 
Feure, J. (1992). Genre study and television. In R. C. Allen (Ed.), Channels of Discourse, Reassembled: Television and Contemporary Criticism (pp. 138-159). London: Routledge.

Finn, J., \& Lavitt, M. (1994). Computer-Based Self-Help Groups for Sexual Abuse Survivors

Social Work with Groups, 17(1/2).

Flaming (Internet). (n.d.). Retrieved May 17, 2007, from http://en.wikipedia.org/wiki/Flame_war

Furnas, G., Fake, C., von Ahn, L., Schachter, J., Golder, S., Fox, K., et al. (2006). Why do tagging systems work? Paper presented at the CHI '06 extended abstracts on Human factors in computing systems.

Furnas, G., Landauer, T., Gomez, L., \& Dumais, S. (1987). The vocabulary problem in human-system communication. Communications of the ACM, 30, 964-971.

Galegher, J., Sproull, L., \& Kiesler, S. (1998). Legitimacy, authority, and community in electronic support groups. Written Communication(15), 493-530.

Giddens, A. (1979). Central problems in social theory. London, England: Macmillan.

Giddens, A. (1986). The Constitution of Society: Outline of the Theory of Structuration (Reprint edition ed.). Berkeley and Los Angeles: University of California Press.

GIST Support International. (n.d.). Retrieved July 23, 2007, from http://www.gistsupport.org/

GIST Support International - Organization. (n.d.). Retrieved July 23, 2007, from http://www.gistsupport.org/gsi-community/about-gsi/organization.php

Glaser, B. G., \& Strauss, A. (1967). The Discovery of Grounded Theory: Strategies for Qualitative Research. New York: Aldine Transaction.

Google Analytics. (n.d.). Retrieved July 23, 2007, from http://www.google.com/analytics/

Grudin, J. (1988). Why CSCW applications fail: problems in the design and evaluation of organization of organizational interfaces. Paper presented at the ACM conference on Computer-supported cooperative work.

Hansen, D. (2003). Who do you trust? The role of expertise in an online support group. Paper presented at the Association of Internet Researchers Conference, Toronto, Ontario.

Hansen, D., Resnick, P., \& Riedl, J. (2005). Online Conversation Design Space available at http://www.socialcomputingresearch.net/workshop/DesignSpace. Unpublished Workshop Paper.

Helgeson, V., \& Cohen, S. (1996). Social support and adjustment to cancer: reconciling descriptive, correlational, and intervention research. Health Psychology, 15, 135148.

Hiltz, S. R., \& Turoff, M. (1993). The network nation: human communication via computer. Cambridge, MA: MIT Press.

Hoch, D., \& Ferguson, T. (2005). What I've learned from E-patients. PLoS Med, 2(8), e206.

House, J. S., Landis, K. R., \& Umberson, D. (1988). Social relationships and health. Science, 241, 540-545. 
Hughes, J., King, V., Rodden, T., \& Andersen, H. (1994). Moving out from the control room: ethnography in system design Paper presented at the ACM conference on Computer supported cooperative work, Chapel Hill, North Carolina.

IP.Board, IPS Community. (n.d.). Retrieved July 23, 2007, from http://www.invisionpower.com/community/board/

Johnson, G. J., \& Ambrose, P. J. (2006). Neo-tribes: the power and potential of online communities in health care. Commun. ACM, 49(1), 107-113.

Joyce, E., \& Kraut, R. (2006). Predicting Continued Participation in Newsgroups. Journal of Computer-Mediated Communication, 11(3).

Karau, S., \& Williams, K. (1993). Social loafing: A meta-analytic review and theoretical integration. Journal of Personality \& Social Psychology, 65(4), 681--706.

Kim, A. J. (1999). Community building on the Web: secret strategies for successful online communities. Berkeley, CA: Peachpit Press.

Kimble, C., Hildreth, P., \& Wright, P. (2001). Communities of practice: Going virtual. In Knowledge management and business model innovation (pp. 220-234). Hershey, PA: Idea Group Publishing.

Klemm, P., Hurst, M., Dearholt, S. L., \& Trone, S. R. (1999). Gender differences on Internet cancer support groups. Computers in Nursing, 17(2), 65-72.

Klemm, P., Reppert, K., \& Visich, L. (1999b). A nontraditional cancer support group: the Internet. Computers in Nursing, 16, 31-36.

Kohn, P. M. (1996). On coping with daily hassles. In M. Zeidner \& N. S. Endler (Eds.), Handbook of Coping (pp. 181-201). New York, NY: John Wiley \& Sons, Inc.

Kollock, P. (1999). The economies of online cooperation: gifts and public goods in cyberspace. In P. Kollock \& M. Smith (Eds.), Communities in Cyberspace (pp. 220-242). London ; New York: Routledge.

Kollock, P., \& Smith, M. A. (1996). Managing the Virtual Commons: Cooperation and Conflict in Computer Communities. In S. Herring (Ed.), Computer-Mediated Communication: Linguistic, Social, and Cross-Cultural Perspectives (pp. 109128). Amsterdam: John Benjamins.

Lakhani, K. R., \& von Hippel, E. (2003). How open source software works: "free" userto-user assistance. Research policy., 32(6), 923.

Lampe, C., \& Johnston , E. (2005). Follow the (slash) dot: effects of feedback on new members in an online community. Paper presented at the International ACM SIGGROUP conference on Supporting group work.

Lampe, C., \& Resnick, P. (2004). Slash(dot) and burn: distributed moderation in a large online conversation space. Paper presented at the SIGCHI conference on Human factors in computing systems.

Lasker, J., Sogolow, E., \& Sharim, R. (2005). The role of an online community for people with a rare disease: Content analysis of messages posted on a primary biliary cirrhosis mailinglist. Journal of medical Internet research, 7(1), 10.

Lave, J., \& Wenger, E. (1991). Situated learning: legitimate peripheral participation. Cambridge [England] ; New York: Cambridge University Press.

Lee, F., Vogel, D., \& Limayem, M. (2003). Virtual Community Informatics: A Review and Research Agenda. The Journal of Information Technology Theory and Application, 5(1), 47-61. 
Leimeister, J. M., Ebner, W., \& Krcmar, H. (2005). Design, Implementation, and Evaluation of Trust-Supporting Components in Virtual Communities for Patients. Journal of Management Information Systems, 21(4), 101-131.

Leimeister, J. M., \& Krcmar, H. (2005). Evaluation of a Systematic Design for a Virtual Patient Community. Journal of Computer-Mediated Communications, 10(4), article 6.

Leuf, B., \& Cunningham, W. (2001). The Wiki way: quick collaboration on the Web. Boston: Addison-Wesley Longman Publishing.

Lie, H. W., \& Bos, B. (1996). Cascading style sheets, level 1: W3C Recommendation 17 Dec 1996. Retrieved July 23, 2007, from http://www.w3.org/TR/REC-CSS1$\underline{961217 . h t m l}$

Ling, K., Beenen, G., Ludford, P., Wang, X., Chang, K., Li, X., et al. (2005). Using social psychology to motivate contributions to online communities. Journal of Computer-Mediated Communication, 10(4).

LISTSERV Introductory List Owner's Guide. (2003). Retrieved May 25, 2007, from http://www.lsoft.com/manuals/1.8e/owner/listowners_intro.pdf

Listserv, L-Soft International. (n.d.). Retrieved July 23, 2007, from http://www.lsoft.com/products/listserv.asp

Lueg, C. (2000). Where is the action in Virtual Communities of Practice? Paper presented at the D-CSCW 2000 German Computer-Supported Cooperative Work Conference "Verteiltes Arbeiten - Arbeit der Zukunft" Munich, Germany.

Mailbag Assistant 3.82. (n.d.). Charmey, Switzerland: Fookes Software.

Malone, T. (1983). How do people organize their desks?: Implications for the design of office information systems. ACM Transactions on Information Systems, 1(1), 99112.

Maloney-Krichmar, D., \& Preece, J. (2005). A multilevel analysis of sociability, usability, and community dynamics in an online health community. ACM Transactions on Computer-Human Interaction (TOCHI), 12(2), 201-232.

Marchionini, G. (1995). Information Seeking in Electronic Environments. Cambridge: Cambridge University Press.

Markus, L. (1987). Towards a "critical mass" theory of interactive media: Universal access, independence, and diffusion. Communication Research, 14, 491-511.

Marshall, C., Shipman, F., \& McCall, R. (1994). Putting digital libraries to work: issues from experience with community memories. Paper presented at the Digital Libraries '94: the first annual Conference on the Theory and Practice of Digital Libraries.

McLaughlin, M. L., Osborne, K. K., \& Smith, C. B. (1995). Standards of conduct on Usenet. In S. Jones (Ed.), Cybersociety (pp. 90-111). Thousand Oaks, CA: Sage.

MediaWiki. (n.d.). Retrieved July 23, 2007, from http://www.mediawiki.org/wiki/MediaWiki

The MediBoard Network. (n.d.). Retrieved July 23, 2007, from http://www.mediboard.com/

MedShelf. (2007). Retrieved July 23, 2007, from http://medshelf.org

Meier, A., Lyons, E. J., Frydman, G., Forlenza, M., \& Rimer, B. K. (2007). How cancer survivors provide support on cancer-related Internet mailing lists. J Med Internet Res, 9(2), e12. 
Metts, S., \& Manns, H. (1996). Coping with HIV and AIDS: The social and personal challenges. In E. B. Ray (Ed.), Communication and disenfranchisement: Social issues and implications (pp. 347-364). Mahwah, NJ: Lawrence Erlbaum Associates.

Miller, J. K., \& Gergen, K. J. (1998). Life on the line: the therapeutic potentials of computer-mediated conversation. Journal of Marital and Family Therapy, 24, 189-202.

n.a. (2000). Patient Groups and the Global Pharmaceutical Industry: URCH Publishing.

Nardi, B., \& O'Day, V. (1999). Information Ecologies: Using Technology with Heart. Cambridge, MA: MIT Press.

Neal, L., Lindgaard, G., Oakley, K., Hansen, D., Kogan, S., Leimeister, J. M., et al. (2006). Online health communities. Paper presented at the CHI '06 extended abstracts on Human factors in computing systems.

Neal, L., Oakley, K., Lindgaard, G., Kaufman, D., Leimeister, J. M., \& Selker, T. (2007). Online health communities. Paper presented at the CHI '07 extended abstracts on Human factors in computing systems.

Net Lingo: The Internet Dictionary. (n.d.). Retrieved July 23, 2007, from http://www.netlingo.com/index.cfm

Neuhauser, L., \& Kreps, G. L. (2003). Rethinking Communication in the E-health Era. Journal of Health Psychology, 8(1), 7-23.

Neus, A. (2001). Managing Information Quality in Virtual Communities of Practice. In E. Pierce \& R. Katz-Haas (Eds.), Proceedings of the 6th International Conference on Information Quality at MIT. Boston, MA: Sloan School of Management.

Nielsen, J. (1993). Usability Engineering. Boston, MA: Academic Press.

Nonnecke, B., \& Preece, J. (2000). Lurker demographics: counting the silent. Paper presented at the Proceedings of the SIGCHI conference on Human factors in computing systems.

Orlikowski, W. J. (1992). The duality of technology: rethinking the concept of technology in organizations. Organization Science, 3(3), 398-427.

Orlikowski, W. J. (1993). Learning from Notes: Organizational Issues in Groupware Implementation. Information Society, 9, 237-250.

Orlikowski, W. J. (2000). Using Technology and Constituting Structures: A Practice Lens for Studying Technology in Organizations. Organization Science, 11(4), 404-428.

Orlikowski, W. J., \& Yates, J. (1994). Genre Repertoire: The Structuring of Communicative Practices in Organizations. Administrative science quarterly., 39(4), 541.

Orlikowski, W. J., Yates, J., Okamura, K., \& Fujimoto, M. (1995). Shaping Electronic Communication: The Metastructuring of Technology in the Context of Use. Organizational Science, 6(4), 423-444.

Pfeil, U., \& Zaphiris, P. (2007). Patterns of empathy in online communication. Paper presented at the SIGCHI Conference on Human Factors in Computing Systems, San Jose, California.

Porter, C. (2004). A Typology of Virtual Communities: A Multi-Disciplinary Foundation for Future Research. Journal of Computer Mediated Communication, 10(1). 
Powazek, D. M. (2002). Design for community: the art of connecting real people in virtual places. Indianapolis, Ind: New Riders.

Pozzebon, M., \& Pinsonneault, A. (2006). IS Theory Website. Retrieved July 23, 2007, from http://www.istheory.yorku.ca/structurationtheory.htm

Preece, J. (1998). Empathic communities: reaching out across the Web. Interactions, 5(2), 32-43.

Preece, J. (1999). Empathic communities: Balancing emotional and factual communication. Interacting with Computers, 12, 63-77.

Preece, J. (1999b). Empathy Online. Virtual Reality, 4(1), 74-84.

Preece, J. (2000). Online communities : designing usability, supporting sociability. New York: John Wiley.

Preece, J. (2004). Etiquette online: from nice to necessary. Communications of the ACM 47(4), 56-61.

Preece, J., \& Maloney-Krichmar, D. (2003). Online Communities: Focusing on sociability and usability In J. Jacko \& A. Sears (Eds.), Handbook of HumanComputer Interaction (pp. 596-620). Mahwah: NJ: Lawrence Erlbaum Associates Inc. Publishers.

Preece, J., Maloney-Krichmar, D., \& Abras, C. (2003). History and emergence of online communities. In B. Wellman (Ed.), Encyclopaedia of Community. Great Barrington, MA: Berkshire Publishing Group and Sage.

Preece, J., Nonnecke, B., \& Andrews, D. (2004). The top five reasons for lurking: improving community experiences for everyone. Computers in Human Behavior, 20(2), 201-223.

Procter, R., Goldenberg, A., Davenport, E., \& McKinlay, A. (1998). Genres in Support of Collaborative Information Retrieval in the Virtual Library. Interacting with Computers, 10, 157-172.

Rainie, L. (2005). "Who uses the internet, what they do, and what it means". Paper presented at the Freedom to Connect Conference.

Resnick, P., Hansen, D., Riedl, J., Terveen, L., \& Ackerman, M. (2005). Beyond Threaded Conversation. Paper presented at the CHI '05 extended abstracts on Human factors in computing systems.

Resnick, P., Iacovou, N., Suchak, M., Bergstrom, P., \& Riedl, J. (1994). GroupLens: An Open Architecture for Collaborative Filtering of Netnews. Paper presented at the Conference on Computer Supported Coorperative Work, Chapel Hill.

Resnick, P., \& Varian, H. (1997). Recommender Systems. Communications of the ACM, 40(3), 56-58.

Rheingold, H. (1993). The virtual community : homesteading on the electronic frontier. Reading, Mass.: Addison-Wesley Pub. Co.

Ridings, C., \& Gefen, D. (2004). Virtual Community Attraction: Why People Hang Out Online. Journal of Computer-Mediated Communication, 10(1).

Rimer, B. K., Lyons, E. J., Ribisl, K. M., Bowling, J. M., Golin, C. E., Forlenza, M. J., et al. (2005). How new subscribers use cancer-related online mailing lists. $J$ Med Internet Res, 7(3), e32.

Rochmis, J. (2002, October 11, 2002). Wired News: A Site for Your Eyes. Wired Magazine. 
Salem, D. A., Bogat, G. A., \& Reid, C. (1997). Mutual help goes on-line. Journal of Community Psychology, 25, 189-207.

Savolainen, R. (1995). Everyday Life Information Seeking: Approaching Information Seeking in the Context of "Way of Life." Library \& Information Science Research, 17(3), 259-294.

Savolainen, R. (2001). "Living encyclopedia" or idle talk? Seeking and providing consumer information in an Internet newsgroup. Library and Information Science Research, 23(1), 67-90.

Shapiro, D. (1994). The limits of ethnography: combining social sciences for CSCW. Paper presented at the ACM conference on Computer supported cooperative work, Chapel Hill, NC.

Sharf, B. F. (1997). Communicating breast cancer on-line: support and empowerment on the Internet. Women and Health, 26, 65-84.

Shaw, B., McTavish, F., Hawkins, R., Gustafson, D. H., \& Pingree, S. (2000). Experiences of women with breast cancer: exchanging social support over the CHESS computer network. J Health Commun, 5(2), 135-159.

Shipman, F., \& Marshall, C. (1999). Formality Considered Harmful: Experiences, EmergingThemes, and Directions on the Use of Formal Representations inInteractive Systems. Computer Supported Cooperative Work, 8(4), 333-352.

Shipman, F., \& McCall, R. (1999). Incremental formalization with the hyper-object substrate. ACM Transactions on Information Systems, 17(2), 199-227

Shneiderman, B. (2000). Designing trust into online experiences. Commun. ACM, 43(12), 57-59.

Simon, H. A. (1996). The Sciences of the Artificial (Third ed.). Cambridge, Massachusetts: The MIT Press.

Smith, A. (1999). Problems of Conflict Management in Virtual Communities. In P. Kollock \& M. Smith (Eds.), Communities in Cyberspace (pp. 107-133). London ; New York: Routledge.

Smith, M. (1999). Invisible crowds in cyberspace: mapping the social stucture of the usenet. In P. Kollock \& M. Smith (Eds.), Communities in Cyberspace (pp. 195219). London ; New York: Routledge.

Smith, M., \& Fiore, A. (2001). Visualization components for persistent conversations. Paper presented at the Conference on Human Factors in Computing Systems, Seattle, WA.

Sproull, L., \& Patterson, J. (2004). Making information cities livable. Communications of the ACM 47(2), 33-37

Star, S., \& Griesemer, J. (1989). Institutional Ecology, 'Translations' and Boundary Objects: Amateurs and Professionals in Berkeley's Museum of Vertebrate Zoology, 1907-39. Social Studies of Science, 19(3), 387-420.

Stein, E., \& Zwass, V. (1995). Actualizing Organizational Memory with Information Technology. Information Systems Research, 6(2), 85-118.

Swales, J. (1990). Genre Analysis: English in Academic Settings. Cambridge: Cambridge University Press.

Swales, J. (1998). Other Floors, Other Voices: a textography of a small university building. Mahwah, NJ: Lawrence Erlbaum Associates. 
Taylor, R. (1968). Question-negotiation and information seeking in libraries. College and Research Libraries 29, 178-194.

Vickery, B., \& Vickery, A. (2004). Information Science in Theory and Practice (Third revised and enlarged edition ed.). München: K.G. Saur.

Viega, J., Warsaw, B., \& Manheimer, K. (1998). Mailman: The GNU Mailing List Manager. Paper presented at the 12th Systems Administration Conference (LISA '98), Boston, Massachusetts.

Walsh, J., \& Ungson, G. (1991). Organizational memory. Academy of Management Review, 16(1), 51-91.

Wasko, M., \& Faraj, S. (2000). "It is What One Does": why people participate and help others in electronic communities of practice. Journal of Strategic Information Systems, 9, 155-173.

Wellman, B. (1997). An electronic group is virtually a social network. In S. Kiesler (Ed.), Culture of the Internet (pp. 179-204). Mahwah, NJ: L. Erlbaum.

Wellman, B., \& Gulia, M. (1999). Net Surfers Don't Ride Alone: Virtual Communities as Communities. In P. Kollock \& M. Smith (Eds.), Communities in Cyberspace (pp. 167-194). London ; New York: Routledge.

Wenger, E. (1998). Communities of practice : learning, meaning, and identity. Cambridge, U.K. ; New York, N.Y.: Cambridge University Press.

Wenger, E., McDermott, R. A., \& Snyder, W. (2002). Cultivating communities of practice: a guide to managing knowledge. Boston, Mass.: Harvard Business School Press.

White, M., \& Dorman, S. M. (2000). Online support for caregivers. Analysis of an Internet Alzheimer mailgroup. Comput Nurs, 18(4), 168-179.

White, M., \& Dorman, S. M. (2001). Receiving social support online: Implications for health education. Health education research, 16(6), 693-707.

Wikgren, M. (2001). Health discussions on the Internet - A study of knowledge communication through citations. Library and Information Science Research, 23(4), 305-317(313).

Wikgren, M. (2003). Everyday health information exchange and citation behaviour in Internet discussion groups. The New Review of Information Behavior Research, 4(1), 225-239.

Wiki. (n.d.). Retrieved July 24, 2007, from http://en.wikipedia.org/wiki/Wiki

Wikipedia: Refactoring Talk Pages. (n.d.). Retrieved June 28, 2006, from http://en.wikipedia.org/wiki/Wikipedia:Refactoring_talk_pages

WikkiTikkiTavi. (n.d.). Retrieved July 23, 2007, from http://tavi.sourceforge.net/

Winzelberg, A. (1997). The Analysis of an Electronic Support Group for Individuals With Eating Disorders. Computers in Human Behavior, 13(3), 393-407(315).

Wright, K. (2000). The communication of social support within an online community for older adults: A qualitative analysis of the SeniorNet community. Qualitative Research Reports in Communication, 1, 33-43.

Wright, K. (2002). Social support within an on-line cancer community: an assessment of emotional support, perceptions of advantages and disadvantages, and motives for using the community from a communication perspective. Journal of Applied Communication Research, 30(3), 195-209. 
Yahoo! Health Groups. (n.d.). Retrieved July 23, 2007, from http://health.dir.groups.yahoo.com/dir/Health_Wellness/Support

Yates, J., \& Orlikowski, W. J. (1992). Genres of Organizational Communication - A Structurational Approach to Studying Communication and Media. Academy of Management Review, 17(2), 299.

Yates, J., \& Orlikowski, W. J. (2002). Genre systems: Structuring interaction through communicative norms. The Journal of Business Communication, 39(1), 13.

Yates, J., Orlikowski, W. J., \& Rennecker, J. (1997). Collaborative Genres for Collaboration: Genre Systems in Digital Media. Paper presented at the 30th Hawaii International Conference of System Sciences (HICSS).

Yin, R. K. (2003). Case Study Research: Design and Methods. Thousand Oaks, CA: Sage Publications.

Zhang, J., Ackerman, M., \& Adamic, L. (2007). Expertise networks in online communities: structure and algorithms. Paper presented at the 16th International Conference on World Wide Web, Banff, Alberta, Canada. 